\title{
Fenomenologia da QCD com saturação de pártons
}

\author{
Andre Veiga Giannini
}

Orientador: Prof. Dr. Francisco de Oliveira Durães Co-orientador: Prof. Dr. Fernando Silveira Navarra

Tese de doutorado apresentada ao Instituto de Física da Universidade de São Paulo como requesito para a obtenção do título de Doutor em Ciências.

Comissão examinadora:

Prof. Dr. Francisco de Oliveira Durães - Mackenzie/IFUSP (orientador)

Prof. Dr. Alessio Mangiarotti - IFUSP

Prof. Dra. Frederique Marie Brigitte Sylvie Grassi - IFUSP

Prof. Dr. Magno Valério Trindade Machado - UfRGS

Prof. Dr. Márcio José Menon - UNICAMP

São Paulo 


\section{FICHA CATALOGRÁFICA}

Preparada pelo Serviço de Biblioteca e Informação do Instituto de Física da Universidade de São Paulo

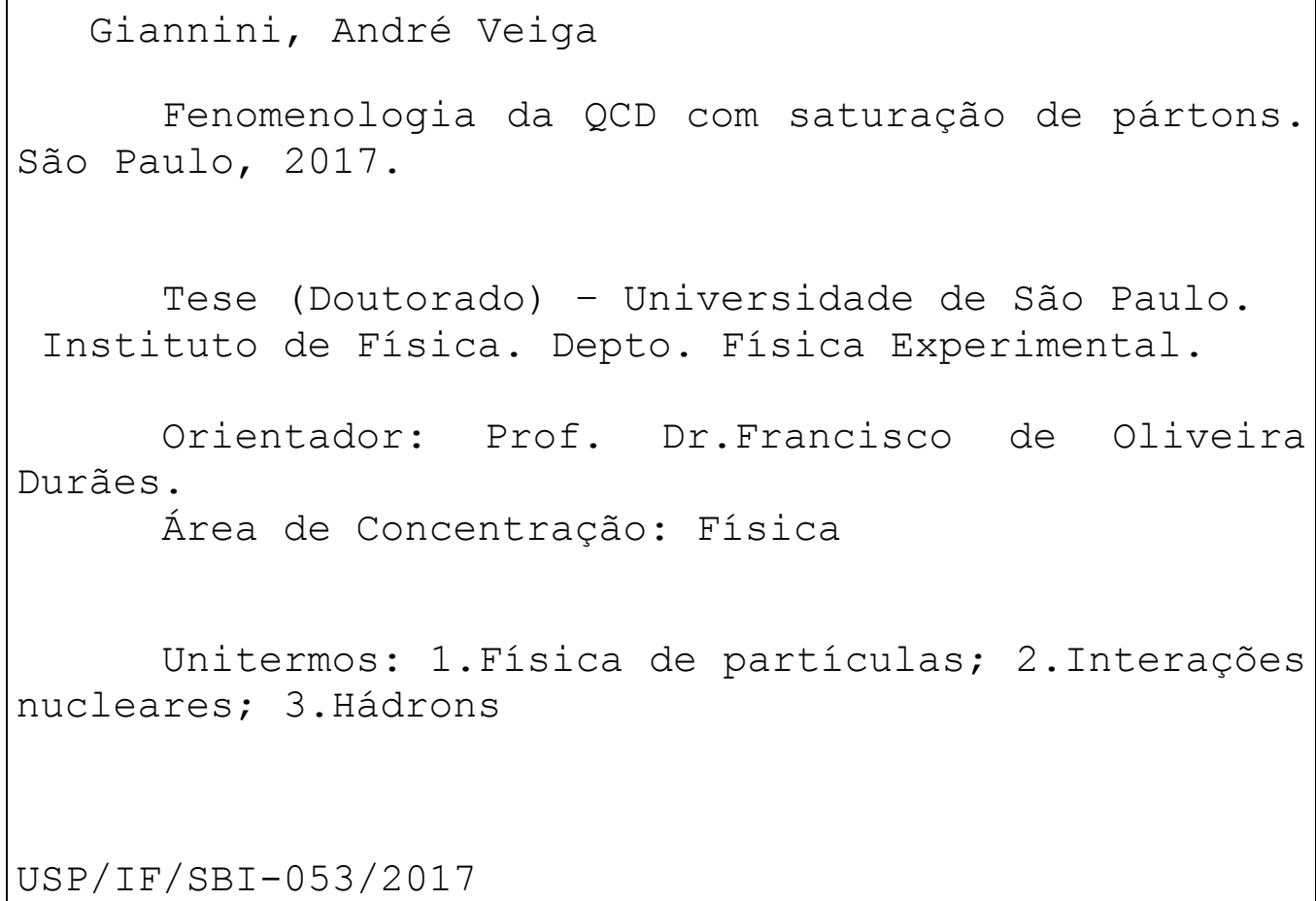




\section{Agradecimentos}

- Agradeço ao professor Francisco de Oliveira Durães pela orientação, pelo incentivo que me fez trilhar o caminho até aqui, pela amizade, paciência e dedicação nesses quase dez anos de trabalho em conjunto. Você foi parte fundamental da minha trajetória, desde o início de meus estudos no Mackenzie e eu sou muito grato por isso. Valeu "véio".

- Agradeço meu co-orientador, professor Fernando Silveira Navarra, também pela orientação e por dividir comigo seus conhecimentos e experiência em física de um modo geral.

- Agradeço ao professor Adrian Dumitru por toda a atenção e supervisão quando da realização de meu estágio no Baruch College. Foi um prazer aprender um pouco de física com você.

- Agradeço ao professor Victor Gonçalves pela colaboração em muitos dos trabalhos que fazem parte desta tese, pelas inúmeras conversas que também ajudaram a encaminhar e a dar forma à essa tese.

- Agradeço à professora Fabiana Carvalho pela colaboração em um dos trabalhos que fazem parte desta tese.

- Agradeço à toda minha família pelo apoio incondicional e por acreditar em mim ao longo de todos esses anos de estudo. Foi a partir do esforço de vocês para que meus irmãos e eu conseguíssemos cursar e concluir uma graduação que, em 
particular, eu cheguei até aqui. Ainda se tratando de família, agradeço à Marina de Castro Fragoso pela companhia inseparável e por tantos bons momentos durante a parte final de meu doutoramento. Sem vocês nada disso seria possível!

- Agradeço a todos os meus amigos pelas conversas e os bons momentos que passamos, eles me faziam esquecer um pouco da pressão (que muitas vezes eu mesmo colocava) de estar cursando um doutorado.

- Agradeço também aos professores e amigos do GRHAFITE pela companhia agradável nesses sete anos de USP.

- Agradeço à FAPESP pelo apoio financeiro durante a realização de meu doutoramento na Universidade de São Paulo (processo 2012/17200-0) e também quando da realização de meu estágio no Baruch College (processo 2013/23848 - 5). 


\section{Resumo}

O estudo das interações fortes a altas energias levou a um grande interesse no regime da QCD onde os pártons carregam uma pequena fração de momento dos hádrons colidentes. Neste regime, tais hádrons podem ser vistos como um meio altamente denso e colorido. Acredita-se hoje que, no domínio de altas energias e/ou altas densidades, a dinâmica da QCD torna-se qualitativamente diferente da dinâmica no regime de curtas distâncias e de altos momentos transferidos. Na linguagem de pártons, essa teoria vem sendo descrita em termos da "saturação" de pártons, resultando na formação de matéria de alta densidade, o Condensado de Vidro de Cor (Color Glass Condensate CGC), onde os efeitos de recombinação de gluons se tornam muito importantes.

Nesse trabalho consideram-se os efeitos da saturação de pártons no estudo de processos hadrônicos e de fotoprodução. Tais efeitos foram incluídos fazendo-se uso de duas diferentes abordagens: inicialmente eles foram introduzidos como correções não lineares de mais altas ordens na constante de acoplamento forte nas equações de evolução para as distribuições de pártons e, posteriormente, através dos chamados modelos de "dipolo de cor" com o uso da chamada "abordagem do CGC".

No primeiro caso estudamos o comportamento energético das seções de choque totais próton-próton (antipróton) $(p p(\bar{p}))$, fóton-próton $(\gamma p)$ e fóton-fóton $(\gamma \gamma)$ e inelástica próton-Ar $(p A r)$ e verificamos que a presença dos efeitos da saturação atenua o crescimento das seções de choque consideradas a altíssimas energias. No segundo caso desenvolvemos cinco outros estudos, sendo os quatro primeiros voltados para a produção de partículas nas regiões central e frontal de rapidez em colisões hadrônicas. Conside- 
rando em um primeiro momento a região frontal de rapidez, estudamos as distribuições de rapidez e em $x$-Feynman referentes à produção líquida de bárions em colisões $p p$, $p A$ e núcleo-núcleo $(A A)$, onde observamos uma violação do scaling de Feynman no espectro em $x_{F}$ de partículas dominantes e, posteriormente, a produção de mésons $D$ em colisões $p p$, onde verificamos que a existência do chamado "charme intrínseco"na função de onda do projétil altera as distribuições em $x_{F}$ de tais mésons em uma região importante para a física de raios cósmicos. Em seguida, apresentamos um estudo sobre o comportamento do momento transversal médio de partículas carregadas produzidas em colisões $p p$ e $p A$ com a rapidez e com a energia da colisão, $\left\langle p_{T}(y, \sqrt{s})\right\rangle$ e mostramos que a razão $R=\left\langle p_{T}(y, \sqrt{s})\right\rangle /\left\langle p_{T}(0, \sqrt{s})\right\rangle$ decresce com a rapidez para uma energia fixa, tendo um comportamento semelhante áquele obtido via modelos hidrodinâmicos.

Com o uso do chamado formalismo da "fatorização $k_{T}$ ", no quarto estudo consideramos a produção de partículas em colisões $p p, p A$ e $A A$ na região central de rapidez e mostramos que as correções na constante de acoplamento forte, incluídas recentemente na seção de choque invariante para a produção de gluons desse formalismo, conduzem a uma dependência energética mais fraca dos observáveis analisados. Finalmente, no quinto estudo apresentamos um simples modelo capaz de gerar as chamadas "assimetrias azimutais" no estado inicial de colisões $p A$ e verificamos que tal modelo descreve qualitativamente bem a dependência com o momento transversal do primeiro harmônico azimutal par e dos dois primeiros harmônicos azimutais ímpares.

Palavras chave: Física de partículas, Interações nucleares, Hádrons. 


\section{Abstract}

The study of the strong interactions at high energies has led to a great interest in the QCD regime where partons carry a small fraction of the colliding hadrons momenta. In this regime these hadrons can be seen as a highly dense and colorful medium. It is believed today that in the domain of high energies and/or high densities the QCD dynamics becomes qualitatively different of the dynamics of the regime of small distances and large momentum transferred. In the parton language this theory is being described in term of the parton "saturation", resulting in the formation of high density matter, the Color Glass Condensate (CGC), where the effects of gluon recombination become very important.

In this work the effects of parton saturation are considered to study hadronic and photonic processes. Such effects were included using two different approaches: initially they were introduced as non linear higher order corrections in the strong coupling constant in the evolution equations for the parton distributions and later through the so called "dipole models" with the use of the "CGC approach".

In the first case we studied the behavior of the proton-proton (antiproton) $(p p(\bar{p}))$, photon-proton $(\gamma p)$ and photon-photon $(\gamma \gamma)$ total cross sections and the proton-Air $(p$ Air) inelastic cross section and verified that the presence of saturation effects tame the growth of the mentioned cross sections at very high energies. In the second case we have developed five other studies, with the first four focused on the particle production in the central and forward rapidity region in hadronic collisions. Considering at first the forward rapidity region we studied the rapidity and $x$-Feynman distributions of the 
net baryon production in $p p, p A$ and nucleus-nucleus $(A A)$ collisions, where a violation of the Feynman scaling in the $x_{F}$ spectra of leading particles has been observed and later the $D$ meson production in $p p$ collisions where we verified that the existence of the so called "intrinsic charm" in the projectile wave function changes the $x_{F}$ distribution of such mesons in an important region for cosmic ray physics. Next we present a study regarding the behavior of the average transverse momentum of charged particles produced in $p p$ and $p P b$ collisions with the rapidity and with the energy, $\left\langle p_{T}(y, \sqrt{s})\right\rangle$, and showed that the ratio $R=\left\langle p_{T}(y, \sqrt{s})\right\rangle /\left\langle p_{T}(0, \sqrt{s})\right\rangle$ decreases with the rapidity for a fixed energy, having a similar behavior to that obtained from hydrodynamic models.

With the use of the so called " $k_{T}$-factorization", in the forth study we have considered the particle production in the central rapidity region in $p p, p A$ and $A A$ collisions and showed that the strong coupling constant correction that were recently introduced in the invariant cross section for gluon production of this formalism, lead to a weaker energy dependence of the analyzed observables. Finally, in the fifth study we presented a simple model capable of generate the so called "azimuthal asymmetries" in the initial-state of $p A$ collisions and verified that this model describes qualitatively well the transverse momentum dependence of the first even azimuthal harmonic and the first two odd azimuthal harmonics.

Keywords: Particle physics, Nuclear interactions, Hadrons. 


\section{Publicações}

Durante o período em que o doutorado foi realizado, os seguintes trabalhos foram submetidos à publicação em revistas indexadas ou como proceedings de conferências:

1. F. Carvalho, A. V. Giannini, V. P. Gonçalves and F. S. Navarra, "D - meson production at very forward rapidities: estimating the intrinsic charm contribution," arXiv:1701.08451 [hep-ph]. Submetido à Physical Review D.

2. F. O. Durães, A. V. Giannini, V. P. Gonçalves and F. S. Navarra, "Testing the running coupling $k_{T}$-factorization formula for the inclusive gluon production," Phys. Rev. D 94, no. 5, 054023 (2016).

3. F. O. Durães, A. V. Giannini, V. P. Gonçalves and F. S. Navarra "Rapidity dependence of the average transverse momentum in hadronic collisions," Phys. Rev. C 94, no. 2, 024917 (2016).

4. F. O. Durães, A. V. Giannini, V. P. Gonçalves and F. S. Navarra, "The color glass condensate and the average transverse momentum in proton-nucleus collisions," J. Phys. Conf. Ser. 736, no. 1, 012025 (2016).

5. F. O. Durães, A. V. Giannini, F. S. Navarra and V. P. B. Gonçalves, "Forward particle production in the CGC formalism: average transverse momentum and $\tau$ scaling," J. Phys. Conf. Ser. 706, no. 5, 052015 (2016).

6. A. Dumitru and A. V. Giannini, "Initial state angular asymmetries in high energy $p+A$ collisions: spontaneous breaking of rotational symmetry by a color electric field and C-odd fluctuations," Nucl. Phys. A 933, 212 (2015).

7. A. Dumitru, A. V. Giannini and V. Skokov, "Anisotropic particle production and azimuthal correlations in high-energy pA collisions," arXiv:1503.03897 [hep-ph].

8. F. O. Durães, A. V. Giannini, V. P. Gonçalves and F. S. Navarra, "Baryon stopping and Feynman scaling in the color glass condensate approach," J. Phys. Conf. Ser. 636, no. 1, 012015 (2015).

9. F. O. Durães, A. V. Giannini, V. P. Gonçalves and F. S. Navarra, "Net baryon production in the CGC formalism," J. Phys. Conf. Ser. 630, no. 1, 012040 (2015).

10. F. O. Durães, A. V. Giannini, V. P. Gonçalves and F. S. Navarra "Baryon stopping in the Color Glass Condensate formalism: A phenomenological study," Phys. Rev. C 89, no. 3, 035205 (2014).

11. A. V. Giannini and F. O. Durães, "Nonlinear effects and the behavior of total hadronic and photonic cross sections," Phys. Rev. D 88, 114004 (2013).

Nesta tese, vamos apresentar os resultados presentes nas referências 1, 2, 3, 6, 10 e 11 . 


\section{Conteúdo}

$\begin{array}{ll}\text { Introdução } & 1\end{array}$

1 Estrutura hadrônica e QCD $\quad 9$

1.1 A cinemática do DIS . . . . . . . . . . . . . . . . . . . . . . 9

1.2 O modelo a pártons e a QCD . . . . . . . . . . . . . . 14

1.2.1 A constante de acoplamento da QCD . . . . . . . . . . . 21

1.3 As equações de evolução da QCD . . . . . . . . . . . . . . . . . . . 24

1.3.1 As equações DGLAP no limite de $x \rightarrow 0$ e $Q^{2} \rightarrow \infty \ldots 29$

1.3 .2 A equação BFKL . . . . . . . . . . . . . . . . . . . . . . 33

2 A Física de Sistemas com Altas Densidades Partônicas 36

2.1 A saturação de pártons . . . . . . . . . . . . . . . . . . 36

2.2 Indícios experimentais da saturação de pártons . . . . . . . . . . . . . . 40

2.2.1 O scaling geométrico . . . . . . . . . . . . . . 43

2.2.2 Observáveis integrados no momento transversal . . . . . . . . . 46

2.2.3 O efeito "ridge" e assimetrias angulares em colisões $p p$ e $p A$. . 49

2.3 O formalismo de dipolo de cor . . . . . . . . . . . . . . . 54

2.3.1 Coordenadas do Cone de Luz . . . . . . . . . . . . . 55

2.3.2 DIS no referêncial de repouso do alvo . . . . . . . . . . . . . 56

2.3.3 O modelo GBW . . . . . . . . . . . . . . . . . 68

2.3.4 Os modelos BUW e DHJ . . . . . . . . . . . . . . 69

2.4 A saturação de gluons segundo as equações de evolução . . . . . . . . 71 
3 A influência da saturação de pártons nas seções de choque hadrônicas $\begin{array}{ll}\text { e fotônicas } & 76\end{array}$

3.1 A seção de choque de minijatos . . . . . . . . . . . . . . . . . . . 77

3.2 Formalismo eiconal . . . . . . . . . . . . . . . . . . . . 82

3.2.1 A aproximação eiconal . . . . . . . . . . . . . . . 82

3.3 Colisões hádron-núcleo no formalismo eiconal: a aproximação de Glauber 87

3.4 Modelo de minijatos eiconalizado com saturação de pártons . . . . . . . 91

3.4.1 Resultados para as seções de choque hadrônicas . . . . . . . . . 96

3.4.2 Resultados para as seções de choque de fotoprodução . . . . . . 104

4 Produção inclusiva de hádrons leves e pesados com efeitos de sa$\begin{array}{ll}\text { turação } & 107\end{array}$

4.1 Produção de partítulas na teoria efetiva do CGC: a fatorização $k_{T}$ e o formalismo híbrido . . . . . . . . . . . . . . . . . . . 108

4.2 Produção líquida de bárions no formalismo do CGC . . . . . . . . . . . 113

4.3 Produção de mésons $D$ em colisões $p p$ : estimando a contribuição do charme intrínseco . . . . . . . . . . . . . . . . . . . 125

4.3.1 Produção de mésons $D$ na região frontal de rapidez . . . . . . . 132

4.3.2 Resultados e discussões . . . . . . . . . . . . . . 136

4.4 O momento transversal médio de partículas em colisões hadrônicas e sua dependência com a rapidez . . . . . . . . . . . . . . 145

4.5 Produção de partículas com a fatorização $k_{T} \ldots$. . . . . . . . . . . . 162

4.5.1 Produção inclusiva de gluons no formalismo da fatorização $k_{T} \quad 164$

4.5.2 Resultados . . . . . . . . . . . . . . . 169

5 Assimetrias angulares em colisões próton-núcleo a altas energias $\mathbf{1 7 6}$

5.1 Correlação de partículas . . . . . . . . . . . . . . 176

5.2 A distribuição de momento de quarks espalhados em colisões $p A$. . . . 182

5.3 Os harmônicos azimutais . . . . . . . . . . . . . . . . . 186

5.4 Modelos para a parte real de $S\left(r, \phi_{r}\right) \ldots \ldots \ldots$. . . . . . . 186 
5.4 .1 Modelo de dipolo quase-clássico . . . . . . . . . . . . 186

5.4.2 Modelo de dipolo com dimensão anômala . . . . . . . . . . . . . 190

5.5 Modelos para a parte imaginária de $S\left(r, \phi_{r}\right) \ldots \ldots$. . . . . . . . . 192

5.6 Resultados . . . . . . . . . . . . . . . . . . . . . . . 197

5.6 .1 Harmônicos pares . . . . . . . . . . . . . . . . . 198

5.6 .2 Modelo de domínio . . . . . . . . . . . . . . 200

5.6.3 Harmônicos ímpares . . . . . . . . . . . . . . . . . . . 203

6 Conclusões 205 


\section{Lista de Figuras}

1.1 Diagrama de Feynman para o DIS. . . . . . . . . . . . . . . . . . . 10

1.2 Medidas de $F_{2}$ realizadas no SLAC. Extraído de [84]. . . . . . . . . . 16

1.3 Dados experimentais da função de estrutura do próton, $F_{2}$ comprovando a violação do scaling de Bjorken. Extraído de [103] . . . . . . . . . . . . 28

1.4 Evoução da estrutura partônica do próton no plano $\left(\ln \left(Q^{2}\right), \ln (1 / x)\right)$. A curva preta denota a chamada "escala de saturação". Figura extraída

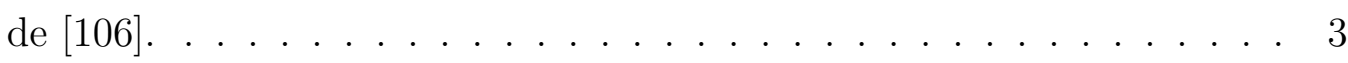

2.1 Distribuição de gluons obtidas a partir dos dados experimentais do HERA [27]. . . . . . . . . . . . . . . . . . . . . . 3

2.2 Seção de choque do DIS para $x<0.01$ (esquerda) e $x>0.01$ (direita) em função da variável $\tau=Q^{2} R_{0}^{2}(x)$ onde $0,045 \mathrm{GeV}^{2}<Q^{2}<450$ $\mathrm{GeV}^{2}$. Os dados experimentais para pequenos valores de $x$ apresentam a propriedade do scaling geométrico enquanto que os dados experimentais para grandes valores de $x$ não apresentam esta propriedade. Figuras extraídas de $[121] \ldots \ldots \ldots \ldots$. . . . . . . . . . . . . . 4

2.3 Propriedade de scaling geométrico observada nos dados experimentais para a distribuição de momento transversal de partítulas carregadas produzidas em (a) colisões $p p$ nas energias do LHC e (b) colisões $A A$ nas energias do RHIC plotadas como função da variável $\tau=\left(p_{T} / Q_{s}\right)^{2}$. Figuras retiradas de $[129,130] \ldots \ldots$. . . . . . . . . . . . . . . 47 
2.4 (a) Comportamento da multiplicidade de partículas carregadas produzidas em $\eta=0$ normalizada pelo número de pares de partículas participantes em colisões $A A$ (curva e pontos experimentais superiores) e em colisões $p p$ (curva e pontos experimentais inferiores) com a energia (b) Distribuição de rapidez em colisões $A u A u$ em $\sqrt{s}=200 \mathrm{GeV}$ para diversas janelas de centralidade. Figuras retiradas de [134] . . . . . . .

2.5 (a) Comportamento da multiplicidade de partículas carregadas produzidas em $\eta=0$ normalizada pelo número de pares de partículas participantes em colisões $\mathrm{PbPb}$ em função do número de participantes. Figura retirada de $[136] . \ldots \ldots \ldots$. . . . . . . . . . . . . . . . . . 50

2.6 Efeito ridge em colisões $p p$ (esquerda), $p P b$ (centro) e $P b P b$ (direita) medidos pela colaboração CMS. O eixo vertical denota a função de

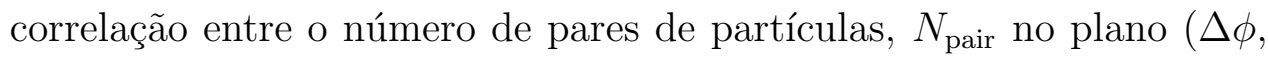
$\Delta \eta)$ normalizada pelo número de partículas que satisfazem o os critérios de seleção aplicados na análise dos dados experimentais, $N_{\text {trig. }}$. Figuras adaptadas de $[139,151,153] \ldots \ldots \ldots \ldots \ldots$

2.7 DIS no formalismo de dipolo de cor. O fóton virtual flutua em um par quark-antiquark que interage com o hádron alvo. . . . . . . . . . . 58

2.8 Amplitude de espalhamento frontal dipolo-alvo, $\mathcal{N}\left(x, r \equiv x_{\perp}\right)$ como função da separação transversa entre quark e anti-quark, $r\left(\equiv x_{\perp}\right.$ nesta figura), que formam um dipolo de cor. Figura extraída de [58]. . . . . .

2.9 Esquema da evolução da distribuição de gluons incorporando o efeito de recombinação durante uma colisão a altas energias. Extraído de [41]. 72

2.10 Esquema das equações de evolução no plano $\ln (1 / x)-\ln Q$ com a respectiva imagem pictórica a nível partônico. Extraído de [206]. . . . . .

3.1 Seção de choque total $p p(\bar{p})$ segundo o modelo de minijatos com $\sigma_{0}=$ 42 mb. Adaptado de [72] . . . . . . . . . . . . . . . . . . . . . . 81

3.2 Espalhamento de uma onda plana por um potencial. Extraído de [101]. 84 
3.3 Um hádron projétil atingindo um núcleo alvo com um parâmetro de impacto $\vec{b}$. O parâmetro de impacto relativo para cada nucleon, $\vec{b}_{i}$, governa o espalhamento hádron-nucleon que ocorre com cada nucleon i. Extraído de $[244] . \ldots \ldots$. . . . . . . . . . . . . . . . 88

3.4 Contribuição da parte não perturbativa da função eiconal em colisões

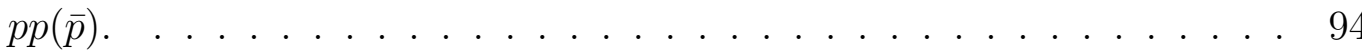

3.5 Seção de choque total $p p$ e $\bar{p} p$. As linhas sólida, tracejada e pontilhada mostram o resultado do modelo eiconal (equação (3.27)) nos regimes não linear e linear. Os dados experimentais são das referências [216-220] 97

3.6 Razões entre as seções de choque total apresentadas na Figura 3.5 e as parametrizações dadas pelas equações (3.54) e (3.55).

3.7 Seção de choque inelástica $p p$ e $p \bar{p}$ As linhas sólida, tracejada e pontilhada mostram o resultado do modelo eiconal (equação (3.29)) nos regimes não linear e linear. Os dados experimentais são das referências [217, $218,220,258-260]$.

3.8 Seção de choque inelástica de produção próton-Ar. As linhas sólida, tracejada e pontilhada mostram o resultado do modelo eiconal nos regimes não linear e linear. Os dados experimentais são das referências [220, $224,244,272-281]$.

3.9 (a) Seção de choque total $\gamma p$. As linhas sólida, tracejada e pontilhada representam os resultados do nosso modelo (equação (3.63)) para diferentes distribuições de pártons. Os dados experimentais são das referências [216,285-287]. (b) Resultados para a seção de choque total $\gamma \gamma$. As linhas sólida, tracejada e pontilhada representam os resultados do nosso modelo (equação (3.64)) para diferentes distribuições de pártons. Os dados experimentais são da referência [288-295]. 
4.1 Distribuição de rapidez da quantidade líquida de bárions produzida em colisões centrais $\mathrm{PbPb}$ no SPS e $A u A u$ no RHIC. Os dados experimentais são das referências $[302-306] \ldots$. . . . . . . . . . . 117

4.2 Distribuição de rapidez para a quantidade líquida de bárions produzida em (a) colisões centrais $P b P b$ e (b) colisões minimum bias $p P b$ nas energias do LHC. . . . . . . . . . . . . . . . . . . . . . . . . 119

4.3 Distribuição de momento transversal da produção líquida de bárions em colisões centrais $A u A u$ nas energias do RHIC para diferentes valores de rapidez. Os dados experimentais são das referências [303-306] . . . . . . 120

4.4 Distribuição de momento transversal de pions (curvas superiores) e prótons (curvas inferiores) produzidos em colisões $\mathrm{PbPb}$ a $\sqrt{s}=2.76$ $\mathrm{TeV}$ e diferentes valores de rapidez.

4.5 Fator de modificação nuclear para a produção líquida de prótons em colisões $p P b, R_{p P b}$ para diferentes valores de energia e rapidez utilizando o modelo BUW

4.6 Dependência com o momento transversal da razão entre as seções de choque de produção líquida de prótons e píons calculada com o modelo BUW em colisões $\mathrm{PbPb}$ para diferentes energias do LHC e diferentes valores de rapidez. . . . . . . . . . . . . . . . . .

4.7 Distribuição de $x$-Feynman para píons e prótons dominantes produzidos em colisões $p p, p A$ e $P b P b$ considerando-se diversas energias de colisão.

4.8 Predições para a dependência com a fração de momento $x$ dos quarks charme (curvas inferiores) e dos gluons (curvas superiores) obtidas pela colaboração CTEQ [344] considerando-se (curvas "BHPS2", "MC2"e "SL2") ou não (curva "no IC") a presença de uma componente IC no próton. . . . . . . . . . . . . . . . . .

4.9 Contribuição para a produção de mésons $D$ na região frontal de rapidez a partir de interações gluon-gluon. . . . . . . . . . . . . . . . . 133 
4.10 Contribuição para a produção de mésons $D$ na região frontal de rapidez a partir de um quark charme no estado inicial. O processo de fragmentação do quark $c / \bar{c}$ em mésons $D$ não é mostrado. . . . . . . . . 135

4.11 Dependência da seção de choque total para a produção de quarks charme com a energia considerando (a) diferentes valores para a massa do charme e (b) diferentes modelos para a componente intrínseca utilizandose $m_{c}=1,4 \mathrm{GeV}$. Os dados experimentais são das referências [367-372]. 139

4.12 Distribuição de rapidez de mésons $D^{0}+\bar{D}^{0}$ produzidos em colisões $p p$ em (a) $\sqrt{s}=7 \mathrm{TeV}$ e (b) $\sqrt{s}=13 \mathrm{TeV}$. Os dados experimentais são das referências $[371,372] \ldots \ldots$. . . . . . . . . . . . . . . . . 140

4.13 Dependência com a rapidez da razão entre os resultados com e sem a componente IC da parametrização CTEQ6.5 nas energias $\sqrt{s}=7$ e 13 TeV considerando (a) o modelo BHPS e (b) o modelo MC. . . . . . . . 140

4.14 Distribuição de $x$-Feynman de mésons $D^{0}+\bar{D}^{0}$ produzidos em colisões $p p$ em $\sqrt{s}=13 \mathrm{TeV}$ considerando a distribuição CTEQ6.5 (a) sem uma componente IC, (b) com modelo BHPS2 para a componente IC e (c) com modelo MC2 para a componente IC. A contribuição gluônica e de charme são apresentadas separadamente. . . . . . . . . . . . . . . . . 142

4.15 Distribuições de $x$-Feynman de mésons $D^{0}+\bar{D}^{0}$ produzidos em colisões pp em (a) $\sqrt{s}=7 \mathrm{TeV}$, (b) $\sqrt{s}=13 \mathrm{TeV}$ e (c) $\sqrt{s}=200 \mathrm{TeV}$ a distribuição CTEQ6.5 sem a componente IC e com a componente IC descrita pelo modelo BHPS2. Os resultados com o modelo SL2 para a componente IC são similares aos resultados "no IC" e os resultados com o modelo MC2 para a componente IC são similares ao resultados do modelo BHPS2. . . . . . . . . . . . . . . . . . . . . 143

4.16 Dependência com $x$-Feynman da razão entre a predição com os modelos para a componente IC e a predição sem a componente IC considerandose (a) o modelo BHPS2 e (b) o modelo MC2 para a componente IC. . . 144 
4.17 Momento transversal médio de partículas produzidas em colisões $p P b$ em função da rapidez, dividido pelo valor do momento transversal médio em $y=0$. Os resultados utilizando-se duas aproximações diferentes no formalismo do CGC, equações (6) e (8) de [375], diferem qualitativamente dos resultados obtidos considerando-se a abordagem hidrodinâmica. Figura extraída de [375] . . . . . . . . . . . . . 148

4.18 Comparação entre as predições dos modelos (a) BUW e (b) DHJ para o espectro de momento transversal de partículas carregadas produzidas em colisões $p P b$ e os dados da colaboração ALICE [297]. Para a nova versão do modelo BUW assumiu-se $K=3.7$ para todas as janelas de pseudorapidez enquanto que para a nova versão do modelo DHJ $K=$ 3.0, 3.0 e 3.7 para $\langle\eta\rangle=0,0.55$ e 1.05 , respectivamente. . . . . . . . 152

4.19 Resultados dos modelos BUW e DHJ para o espectro de momento transversal de partículas carregadas em colisões $p p$. (a) Comparação com os dados da colaboração ALICE [385]. (b) Comparação com os dados da colaboração ATLAS $[386,387]$. . . . . . . . . . . . . . . 15

4.20 Resultados dos modelos BUW e DHJ para (a) o espectro de momento transversal de partículas carregadas em colisões $p p$ comparados com os dados da colaboração CMS [388-390] e (b) para o espectro de momento transversal de píons neutros produzidos em colisões $p p$ comparados com os dados da colaboração ALICE [398] . . . . . . . . . . . . . . . . . 155 
4.21 (a) Resultados dos modelos BUW e DHJ para o espectro de momento transversal de píons neutros produzidos em colisões $p p$. Os fatores $K=$ 1.5 para $\langle\eta\rangle=0.5$ e $K=1.2$ para $\langle\eta\rangle=1.4,3.25,3.7,3.925$ foram utilizados para os resultados com o modelo BUW. Para o modelo DHJ utilizou-se $K=1.2$ para $\langle\eta\rangle=3.25,3.7,3.925$. Os dados experimentais são da referência [399]; (b) Resultados dos modelos BUW e DHJ para o espectro de momento transversal de hádrons produzidos em colisões $d A u$. Os fatores $K=2,9,2,5,2,0,1,0$ e 1, 0 for $\eta=0,1,2,2,3,2$ e 4, respectivamente, foram utilizados nos resultados com o modelo BUW. Para o modelo DHJ tem-se que $K=2,5,2,4,1,5$ para $\eta=2,2,3,2$ e 4, respectivamente. Os dados experimentais são da referência [400-402]. 156

4.22 Comparação entre o resultado do modelo BUW (com os parâmetros originais da referência [205] e os parâmetros novos) e os dados experimentais para a seção de choque total $\gamma^{*} p[23,24,27,30,32,403,404]$ medidos a partir de colisões ep no HERA. . . . . . . . . . . . . 157

4.23 Dependência da razão $\left\langle p_{T}(y, \sqrt{s})\right\rangle /\left\langle p_{T}(0, \sqrt{s})\right\rangle$ com relação à amplitude de espalhamento frontal dipolo-hádron em colisões $p p$ e $p P b$. Os resultados dos painéis (a) e (c) foram obtidos desconsiderando-se o processo de fragmentação enquanto os resultados apresentados nos painéis (b) e (d) incluem este processo através da distribuição KKP. . . . . . . . . . 158

4.24 Dependência da razão $\left\langle p_{T}(y, \sqrt{s})\right\rangle /\left\langle p_{T}(0, \sqrt{s})\right\rangle$ com o valor do momento transversal mínimo, $p_{T, \text { min }}$, considerando (a) o modelo GBW e (b) o modelo BUW para a amplitude de espalhamento frontal. . . . . . . . . 159

4.25 Dependência com a rapidez da razão $R=\left\langle p_{T}(y, \sqrt{s})\right\rangle /\left\langle p_{T}(0, \sqrt{s})\right\rangle$ em (a) colisões $p p$ e (b) colisões $p P b$ para diferentes energias. As predições com o modelo BUW (DHJ) são representadas pelas curvas azuis (vermelhas). . . . . . . . . . . . . . . . . . . 160 
4.26 Dependência com a rapidez da razão entre as predições para $R=$ $\left\langle p_{T}(y, \sqrt{s})\right\rangle /\left\langle p_{T}(0, \sqrt{s})\right\rangle$ em colisões $p p$ e colisões $p P b$ considerando os modelos de dipolo (a) DHJ e (b) BUW. . . . . . . . . . . . . . 160

4.27 Distribuições de pseudorapidez para a produção inclusiva de hádrons em colisões $p p$ para diferentes valores de $\sqrt{s}$ : (a) $0,9 \mathrm{TeV}$, (b) 2,36 TeV e (c) $7 \mathrm{TeV}$. Os dados experimentais são das referências $[388,389,420]$.

4.28 Distribuições de pseudorapidez para a produção inclusiva de hádrons em (a) colisões $d A u(\sqrt{s}=0.2 \mathrm{TeV})$ e (b) colisões $p P b(\sqrt{s}=5.02$ TeV). Os dados experimentais são das referências [421-423]. . . . . . . 173

4.29 Distribuições de pseudorapidez para a produção inclusiva de hádrons em colisões $A u A u$ em $\sqrt{s}=0.2 \mathrm{TeV}$ (curvas inferiores) e em colisões $\mathrm{PbPb}$ em $\sqrt{s}=2.76 \mathrm{TeV}$ (curvas superiores). Os dados experimentais

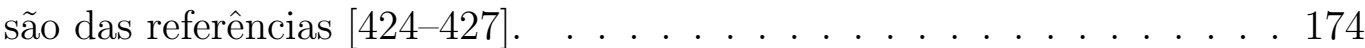

5.1 Esquema tridimensional de uma colisão AA não central. O volume em forma de amêndoa representa as partículas participantes na colisão; na forma em azul encontram-se os nucleons que não participam da colisão ("espectadores") e seguem em sentidos opostos. O plano de reação é formado por $x$, que esta orientado na direção do parâmetro de impacto $(\vec{b})$ e $z$, a direção de propagação do feixe. Extraído de [149]. . . . . . . 178

5.2 Configurações do campo elétrico de cor típicas em um núcleo. Cada região representa um "domínio" diferente de $\vec{E}$. Extraído de [170] . . . . 181

$5.3 v_{2}\left(p_{T}\right)$ obtido com o modelo de dipolo quase-clássico com amplitude de polarização do campo elétrico de cor $\mathcal{A}=7 \%$ (quadrados abertos). Os dados da colaboração CMS, $v_{2}\{2\}$ e $v_{2}\{4\}$, representados por cículos e quadrados cheios, respectivamente, correspondem a colisões $p P b$ com maior multiplicidade a $\sqrt{s}=5.02 \mathrm{TeV} \ldots \ldots . \ldots . \ldots 199$ 
$5.4 v_{2}\left(p_{T}\right)$ a partir da correlação de duas e quatro partículas. O modelo de domínio com $N_{D}=2,95,4,33,5,93$ (e, correspondentemente, $\mathcal{A}=0,15$, 0,20, 0,25) é comparado com os dados da colaboração CMS. . . . . . . 201

$5.5 v_{4}\left(p_{T}\right)$ a partir da correlação de duas e quatro partículas. No painel esquerdo o modelo de domínio com $N_{D}=2,95,4,33,5,93$ (e, correspondentemente, $\mathcal{A}=0,15,0,20,0,25$; de baixo para cima) é comparado com $v_{4}\{2\}$ medido pela colaboração ATLAS. No painel direito encontra-se a predição do modelo para $v_{4}\{4\}$ com as mesmas combinações de $\mathcal{A}$ e $N_{D} \cdot 202$

$5.6 v_{1}\left(p_{T}\right)$ e $v_{3}\left(p_{T}\right)$ obtido com o modelo quase-clássico para $O(\vec{r})$ com uma distribuição de flutuações de paridade de carga ímpar invariante de escala com um corte $Q_{c}$. . . . . . . . . . . . . . . . . 203

$5.7 v_{1}\left(p_{T}\right)$ e $v_{3}\left(p_{T}\right)$ obtidos com o modelo quase-clássico para $O(\vec{r})$ com uma amplitude de flutuação $\mathcal{B}=1.6$ e corte $Q_{c} / Q_{s}=18$. Os dados das colaborações ATLAS [183] $\left(v_{1}\right)$ e CMS [184] $\left(v_{3}\right)$ para $v_{1,3}\{2\}$ correspondem a eventos $p P b$ com a mais alta multiplicidade em $\sqrt{ } s=5.02 \mathrm{TeV}$. . . 204 


\section{Introdução}

De acordo com o presente conhecimento, a matéria, como hoje a concebemos, é constituída por partículas elementares, conhecidas como quarks e léptons. Dentre as quatro forças fundamentais encontradas na natureza, a saber, a eletromagnética, a fraca, a forte e a gravitacional, as três primeiras são fundamentais no estudo da dinâmica dessas partículas. Embora o último processo de interação entre essas partículas ainda não possua um entendimento plausível e consistente, acredita-se que, igualmente, cumprirá um papel determinante nesse conhecimento. Por essa razão, vamo-nos eximir aqui de tratá-lo.

Quarks, léptons e suas interações, que ocorrem devido à troca de partículas denominadas "bósons", são descritas atualmente através do chamado "Modelo Padrão" [1-13]. O Modelo Padrão pode ser dividido em dois setores principais, o eletrofraco e o forte. No primeiro setor, que unifica a descrição das interações eletromagnética e fraca, encontram-se os léptons e os diferentes mediadores dessas interações (o fóton para interação eletromagnética e os bósons $W^{ \pm}$e $Z^{0}$ para a interação fraca). No segundo setor encontram-se os quarks e os gluons (os bósons mediadores da interação forte), formando os hádrons.

O setor forte é de extrema importância para esta tese. Diferente das partículas do setor eletrofraco, quarks e gluons carregam um grau de liberdade adicional, a denominada "carga elétrica de cor". Por esse motivo, a teoria aceita atualmente para descrever as interações entre quarks e gluons é denominada Cromodinâmica Quântica (Quantum Chromodynamics - QCD) [3,4,10-13]. 
A QCD é uma teoria não abeliana, que considera que os mediadores da interação entre esses constituintes, isto é os gluons, podem interagir diretamente uns com os outros, diferentemente da teoria abeliana, onde fótons não interagem entre si. Uma característica de suma importância da QCD é que a constante de acoplamento da teoria, $\alpha_{s}$, tende a zero $\left(\alpha_{s} \ll 1\right)$ no limite de pequenas distâncias, ou equivalentemente, no limite de altas energias ou momentos transferidos. Esta propriedade, denominada "liberdade assintótica" [11-13], permite que neste regime possamos nos valer de métodos perturbativos para calcular quantidades de interesse através da chamada "QCD perturbativa" (perturbative QCD - pQCD).

Por outro lado, a distâncias maiores, ou seja, a energias menores, a constante de acoplamento se torna grande $\left(\alpha_{s} \sim 1\right)$ e, portanto, há um aumento da intensidade da interação entre os constituintes que confina quarks e gluons no interior dos hádrons. Esta propriedade, conhecida como "confinamento" [14], exclui a possibilidade de observarmos quarks e gluons isoladamente na natureza. Disso decorre que, uma vez que apenas hádrons chegam aos detectores experimentais, não é possível ter acesso direto às distribuições de quarks e gluons que os compõem via primeiros princípios.

A estrutura dos hádrons a pequenas escalas de distância pode, no entanto, ser analisada utilizando-se a pQCD devido à propriedade da liberdade assintótica, através do espalhamento inelástico profundo (deep inelastic scattering - DIS). No DIS, um próton e um elétron são acelerados a altas energias e colidem. A interação entre essas partículas é mediada, predominantemente, pela troca de um fóton virtual. Sendo $Q^{2}$ a virtualidade desse fóton, a resolução espacial provada pelo processo é, a grosso modo, dada por $1 / Q^{2}$. Se $Q^{2}$ é grande, os resultados são bem entendidos em termos de um espalhamento único entre o fóton e um quark ou gluon dentro do próton.

Desde antes da formulação da QCD, as partículas constituintes dos hádrons eram conhecidas como "pártons" e suas densidades conhecidas como "funções de distribuição de pártons"(Parton Distribution Functions - PDFs). As PDFs dependem não só de $Q^{2}$ mas também da fração de momento longitudinal do próton, $x$ (de Bjorken), carregada pelo párton espalhado. 
As PDFs carregam informações da física de longas distâncias e, portanto, não podem ser calculadas via primeiros princípios através da pQCD. Sua obtenção se dá através da análise simultânea de diversos conjuntos de dados experimentais. Todavia, uma vez conhecida a PDF para cada tipo de párton em um dado valor inicial $Q_{0}^{2}$, é possível obter sua evolução com a variável $Q^{2}$ através das equações de DokshitzerGribov-Lipatov-Altarelli-Parisi (DGLAP) [15-18], que são derivadas com o uso da pQCD. Por outro lado, mantendo-se $Q^{2}$ fixo, é possível determinar sua evolução com a variável $x$ através da equação de Balitsky-Fadin-Kuraev-Lipatov (BFKL) [19-21], uma vez conhecidas tais distribuições para um dado valor inicial $x_{0}$. Por envolverem apenas termos lineares dessas PDFs, diz-se que as equações DGLAP e BFKL são equações de evolução lineares nessas quantidades.

A nova geração de colisores de altas energias, começando com o Hadron-ElektronRing-Anlage (HERA) na Alemanha e o Tevatron nos Estados Unidos no início dos anos 1990, tendo seu avanço com a construção do Relativistic Heavy Ion Collider (RHIC) a partir do ano 2000 nos Estados Unidos e a partir do ano 2008 com a construção do Large Hadron Collider (LHC) na Suiça, abriu uma nova era nos estudos das interações fortes. Com o progressivo aumento da energia do centro de massa das colisões realizadas nesses colisores, $\sqrt{s}$, valores cada vez menores de $x$ passaram a ser alcançados e têm permitido um melhor conhecimento acerca do comportamento das distribuições de pártons nos hádrons. Em particular, os resultados experimentais advindos do HERA [22-32] confirmaram a previsão das equações DGLAP de que, a altas energias, a distribuição de gluons cresce fortemente a medida que $Q^{2}$ aumenta e/ou $x$ diminui. Esses resultados mostraram que, nesse regime, a contribuição dominante dos processos em nível partônico vem de espalhamentos envolvendo gluons com pouca fração de momento e que o forte crescimento da distribuição de pártons (predominantemente gluons) faz com que os hádrons colidentes sejam vistos como um meio altamente denso e colorido.

No entanto, os estudos que foram e têm sido efetuados acerca do comportamento das distribuições de gluons de baixos momentos nos colisores a altas energias revelaram 
que as equações de evolução DGLAP e BFKL, que incluem apenas processos de emissão de pártons, deveriam ser modificadas de modo que os processos de recombinação de gluons (consequência da alta densidade de gluons alcançada em colisões hadrônicas a altíssimas energias) fossem igualmente considerados [33-57]. É claro, a inclusão desses processos de recombinação nas equações de evolução dão origem a termos não lineares nas distribuições de gluons fazendo, portanto, com que as equações que regem a evolução das distribuições de pártons nesse regime se tornem igualmente não lineares.

O entendimento que hoje temos, no domínio de altas energias e/ou altas densidades, é que a dinâmica da QCD torna-se qualitativamente diferente da dinâmica no regime de curtas ditâncias e de altos momentos transferidos. Na linguagem de pártons, ela vem sendo descrita em termos da "saturação" de gluons resultando na formação de matéria de alta densidade, o Condensado de Vidro de Cor (Color Glass Condensate CGC) [33-58], onde os efeitos de recombinação se tornam muito importantes. Este é o regime que explora a não linearidade da QCD, de alta densidade de pártons e de fortes campos de cor, no qual as amplitudes de transição são dominadas por configurações de campo clássico contendo um grande número de gluons.

A saturação de gluons emerge de um cenário bastante simples e pode ser construído a partir de argumentos geométricos. Em colisões de íons relativísticos, por exemplo, quando um núcleo (de $A$ nucleons) sofre contração de Lorentz, os pártons contidos nos nucleons se distribuem sobre uma fina casca no plano transversal. Pelo princípio da incerteza cada párton (de momento transversal $k_{T}$ ) ocupa uma área transversal $\pi / k_{T}^{2}$, e podem ser "provados" através da seção de choque $\sigma \sim \pi \alpha_{s}\left(k_{T}^{2}\right) / k_{T}^{2}$, onde $\alpha_{s}\left(k_{T}^{2}\right)$ representa a constante de acoplamento (forte) do processo.

Uma vez que a área transversal total do núcleo é $S_{A} \sim \pi R_{A}^{2}$, onde $R_{A}$ é seu raio transversal, quando o número de pártons excede a quantidade $N \sim S_{A} / \sigma \sim$ $k_{T}^{2} R_{A}^{2} / \alpha_{s}\left(k_{T}^{2}\right)$ esses pártons se sobrepõem no plano tansversal e começam a interagir uns com os outros, impedindo assim um posterior crescimento da densidade de pártons. Isso acontece quando o momento transversal for da ordem de $Q_{s}^{2} \sim \alpha_{s}\left(k_{T}^{2}\right) N / R_{A}^{2} \sim$ $A^{1 / 3}$, que é denominada "escala de saturação". O fenômeno da saturação introduz 
assim uma nova escala $\left(Q_{s}\right)$ responsável por identificar os diferentes regimes da QCD: na região $k_{T}<Q_{s}$ encontra-se o regime de saturação, onde os efeitos de recombinação são importantes e as equações de evolução se tornam não lineares; já para $k_{T}>Q_{s}$ os efeitos da saturação de pártons são fracos e as equações de evolução se reduzem a equações lineares nas distribuições de pártons.

Um dos principais objetivos da física hadrônica de altas energias atualmente é procurar sinais da formação do CGC. Várias possíveis indicações experimentais deste fenômeno encontram-se na literatura e podem ser encontradas em [59]. Entretanto, uma série de trabalhos mostram que um mesmo conjunto de dados experimentais também pode ser descrito sem levar em conta os efeitos da saturação [60-63]. Espera-se que o LHC, ou um futuro colisor elétron-íon (cuja a contrução está em discussão) possam fornecer evidências mais convincentes sobre o fenômeno da saturação e a formação do CGC.

Nesta tese utilizamos a teoria da QCD no estudo de processos hadrônicos e de fotoprodução levando em consideração os efeitos da saturação de pártons. Aqui, tais efeitos foram considerados fazendo-se o uso de duas abordagens diferentes, porém complementares.

Em uma primeira aproximação, os efeitos da saturação de pártons foram incluídos como correções de mais altas ordens em $\alpha_{s}$ às equações DGLAP. Os primeiros termos dessas correções, que foram calculados por Gribov, Levin e Riskin [33] e Mueller e Qiu [34], deram origem às equações GLRMQ. Essa aproximação, porém, é válida apenas nas vizinhanças do regime de saturação, já que dentro deste regime é necessario considerar múltiplos espalhamentos do projétil devido a alta densidade de gluons. Levar em conta múltiplos espalhamentos, nesta abordagem, significa a inclusão de um número cada vez maior de correções de mais altas ordens em $\alpha_{s}$, o que é inviável devido ao grande número de diagramas a serem considerados e também a complexidade envolvida nos cálculos.

No entanto, os efeitos da saturação também podem ser incluídos fazendo-se uso dos chamados modelos de "dipolo de cor" [64-69]. Tais modelos tem origem no estudo 
do DIS no referêncial de repouso do alvo e no limite em que $x \rightarrow 0$. Nessas condições, o fóton virtual trocado no processo de espalhamento pode flutuar em um par quarkantiquark, formando um dipolo de cor que interage com o próton (ou núcleo) alvo através de múltiplos espalhamentos. O espalhamento do dipolo pelo alvo é descrito pela chamada "amplitude de espalhamento de dipolo" e, neste cenário, a saturação da densidade de gluons implica na saturação da amplitude de dipolo.

Esta tese está organizada da seguinte maneira: os dois primeiros capítulos possuem o objetivo de apresentar os conceitos fundamentais, principais ferramentas teóricas por nós utilizadas e também especificar em qual regime da física de altas energias foi considerado.

Mais especificamente, no primeiro capítulo apresentamos os principais tópicos da QCD que aqui utilizaremos, com o fim exclusivo de ressaltar a necessidade da inclusão dos efeitos de saturação. Uma importante parte deste capítulo diz respeito às equações de evolução padrão da QCD, as equações DGLAP e BFKL, e a indicação da necessidade de um mecanismo para conter o crescimento das distribuições de pártons a altas energias.

O segundo capítulo encontra-se voltado à caracterização da física que rege os sistemas hadrônicos que contém uma alta densidade partônica. Iniciamos esse capítulo com uma discusão sobre o conceito de saturação de pártons e apresentamos alguns indícios experimentais que corroboram com a existência de tal fenômeno e, portanto, para a formação do CGC. Posteriormente, apresentamos o formalismo de dipolo de cor, onde três diferentes modelos para a amplitude de dipolo são utilizados em nossos estudos, fazendo menção aos principais aspectos e ingredientes nas diferentes abordagens do CGC aqui utilizadas.

No terceiro capítulo apresentamos os tópicos da QCD referentes à construção de um modelo para a descrição das seções de choque totais próton-próton (antipróton) $(p p(\bar{p}))$, próton-núcleo $(p A)$, fóton-próton $(\gamma p)$ e fóton-fóton $(\gamma \gamma)$. Com esse objetivo, introduzimos a seção de choque de produção de minijatos e uma classe de modelos inspirados em QCD baseados no chamado "formalismo eiconal". Os efeitos da sa- 
turação de pártons foram incluídos como correções de mais alta ordem nas equações DGLAP fazendo-se uso das distribuições de pártons EHKQS [70,71], cuja a evolução é regida pelas equações GLRMQ. A escrita desses três primeiros capítulos dessa tese é baseada na referência [72] e tratam também de importantes tópicos não presentes nessa referência.

O quarto capítulo dessa tese é dedicado ao estudo da produção de partículas em colisões hadrônicas a altas energias nas regiões central e, principalmente, frontal de rapidez. Como ponto de partida, apresentamos o ferramental teórico da abordagem do CGC nessas colisões [35-43,57,58,73-75], os chamados "formalismo híbrido" e "formalismo da fatorização $k_{T}$ ", bastante utilizados nesse capítulo. Em ambos os casos os efeitos da saturação de pártons são considerados através dos modelos de dipolo de cor apresentados no capítulo 2. Nas subseções 4.2 e 4.3 estudamos, entre outros observáveis, as distribuições de rapidez (y) e em momento longitudinal (ou $x$-Feynman) da quantidade líquida de bárions (isto é, o número de bárions menos o número de antibárions produzidos) e de mésons $D$, respectivamente. Na subseção 4.4, apresentamos um estudo sobre a dependência do momento transversal médio de partículas produzidas em colisões $p p$ e $p A$ com a energia da colisão e com a rapidez. Os três estudos acima mencionados foram desenvolvidos com o uso do formalismo híbrido na abordagem do CGC. Por fim, apresentamos uma primeira estimativa sobre o impacto das correções em $\alpha_{s}$ incluídas na seção de choque de produção de gluons da fatorização $k_{T}$ [76] através do estudo das distribuições de pseudorapidez e multiplicidade de hádrons carregados produzidos na região central de rapidez.

O quinto capítulo trata das chamadas "assimetrias angulares" medidas em colisões $p A$ a altas energias, onde apresentamos um estudo de cunho qualitativo e exploratório acerca do surgimento de tais assimetrias através da anisotropia gerada no estado inicial da colisão, devido à flutuação das cargas de cor no núcleo alvo. Os ingredientes utilizados, bem como as ideias fundamentais que suportam o modelo proposto são apresentadas, assim como os resultados por nós obtidos.

Por fim, apresentamos as principais conclusões dos estudos acima mencionados e as 
perspectivas futuras que procuraremos almejar. Os resultados dos diferentes estudos apresentados nos capítulos 3, 4 e 5 dessa tese deram origem às publicações [77-82]. 


\section{Capítulo 1}

\section{Estrutura hadrônica e QCD}

Neste capítulo apresentamos os princípios que servem de base para o desenvolvimento desta tese: o espalhamento inelástico profundo (deep inelastic scattering - DIS), o modelo a pártons e, por fim, a QCD e suas equações de evolução.

Este capítulo inicia-se com uma descrição sucinta do processo de DIS, onde as principais equações e suas implicações são apresentadas. A seguir, trata-se do modelo a pártons, que é ponto de partida para a formulação da QCD. O próximo passo será considerar a constante de acoplamento da QCD e mostrar que seu valor é muito pequeno no limite de pequenas distâncias, ou equivalentemente, altas energias ou momentos transferidos e, por conta disso, é possível aplicar a pQCD como ferramenta de cálculo. Por fim, apresentam-se as equações de evolução padrão da QCD conhecidas como Dokshitzer-Gribov-Lipatov-Altarelli-Parisi (DGLAP) e Balitsky-Fadin-KuraevLipatov (BFKL) e discute-se a implicação dos resultados por elas gerados no limite de altas energias.

\subsection{A cinemática do DIS}

O DIS é um processo cujo estudo é de grande importância pois é a ferramenta, a 
nível experimental, que possibilita extrair informações sobre a estrutura interna dos hádrons de um modo mais direto e limpo e, portanto, serve de teste para a teoria que se propõe a descrever a física das interações hadrônicas, a QCD. A compilação da informação sobre a estrutura interna dos hádrons dá origem às distribuições de pártons (Parton Distribution Functions - PDFs), que são um ingrediente fundamental do modelo a pártons e também no estudo de processos apresentados nesta tese.

Nesse processo um lépton, $l$, é utilizado como prova para sondar o interior dos hádrons, geralmente um nucleon, $N$,

$$
l+N \rightarrow l+X
$$

que ao colidir inelasticamente produz um sistema $X$.

Assumindo-se que o processo dominante para este espalhamento ocorre via a troca de um fóton virtual ${ }^{1}$, o diagrama de Feynman mais simples associado a este processo é apresentado na Figura 1.1. Neste processo, $k$ e $k^{\prime}$ são os quadri-momentos inicial e final do lépton, $P$ é o quadri-momento do nucleon alvo e $q=k^{\prime}-k$ o quadri-momento do fóton virtual.

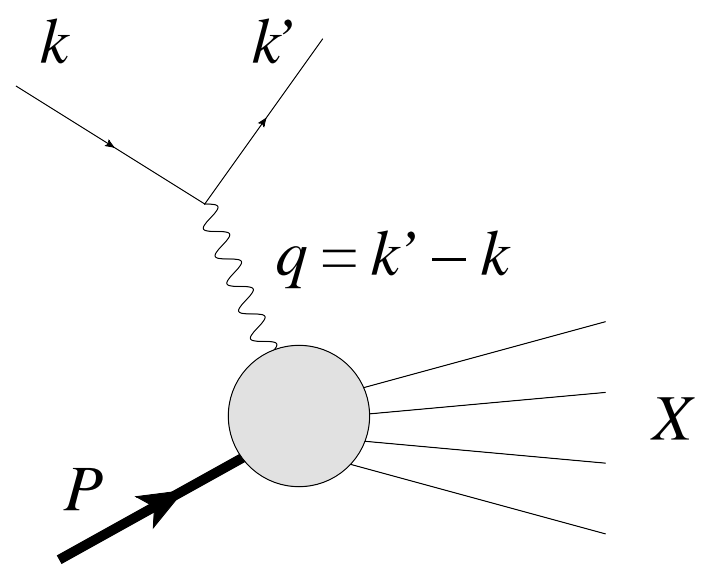

Figura 1.1: Diagrama de Feynman para o DIS.

\footnotetext{
${ }^{1}$ Este mesmo processo pode ocorrer via troca de um dos bósons do setor fraco, $W^{ \pm}$e $Z^{0}$. Conforme o valor do momento transferido na reação aumenta, essas contribuições se tornam importantes e não podem ser desprezadas [57].
} 
A cinemática do DIS é caracterizada por duas variáveis invariantes de Lorentz independentes: a chamada "virtualidade" do fóton, $q^{2} \equiv-Q^{2}$ e o quadrado da massa do sistema hadrônico $X$ produzido, $W^{2}=(P+q)^{2}$. A energia, no referencial do centro de massa, em que este processo ocorre é identificada com a variável de Mandelstan, $s=(k+P)^{2}$.

Considerando-se somente eventos onde o nucleon é um alvo fixo, $P=\left(m_{N}, \mathbf{0}\right)$ e em que as energias inicial e final do lépton, $E$ e $E^{\prime}$ respectivamente, são muito maiores que a sua massa, $m_{N}$, de modo que esta possa ser desprezada, as variáveis que descrevem a cinemática do DIS $\left(s, Q^{2}\right.$ e $\left.W^{2}\right)$ podem ser reescritas como:

$$
\begin{gathered}
Q^{2} \approx 4 E E^{\prime} \sin \frac{\theta}{2}, \\
s=2 E m_{N}+m_{N}^{2}, \\
W^{2}=m_{N}^{2}+2 m_{N} \nu+q^{2},
\end{gathered}
$$

onde $\nu=E-E^{\prime}$ é a energia transferida do lépton para o nucleon através da troca do fóton virtual, $m_{N}$ é a massa do nucleon e $\theta$ é o ângulo de espalhamento do lépton no referencial do laboratório.

Outra alternativa para descrever a cinemática do DIS vem da introdução das variáveis adimensionais

$$
\begin{gathered}
x=\frac{Q^{2}}{2 P \cdot q}=\frac{Q^{2}}{2 m_{N} \nu}=\frac{Q^{2}}{W^{2}+Q^{2}-m_{N}^{2}}, \\
y=\frac{P \cdot q}{P \cdot k}=\frac{W^{2}+Q^{2}-m_{N}^{2}}{s-m_{N}^{2}},
\end{gathered}
$$

onde $y=\nu / E$ é a fração de energia transferida do lépton para o nucleon através do fóton emitido e a variável $x$ é conhecida como " $x$ de Bjorken", que assumem os valores entre $0 \leq x, y \leq 1$ para $W^{2} \geq m_{N}^{2}$.

A seção de choque mais simples a ser medida no DIS é a seção de choque inclusiva. Eventos inclusivos são aqueles em que se mede o momento final do projétil (um lépton 
no caso do DIS) mas os estados hadrônicos finais, indicados por $X$ na Figura 1.1, não são identificados experimentalmente [83]. Uma vez que os estados finais não são conhecidos, a seção de choque inclusiva total é composta pela adição da seção de choque associada a cada estado final possível de $X$ :

$$
E^{\prime} \frac{d \sigma_{l N}}{d^{3} \vec{k}^{\prime}}=\sum_{\text {estados } X} E^{\prime} \frac{d \sigma_{l N \rightarrow l X}}{d^{3} \vec{k}^{\prime}}
$$

Para o caso não polarizado, a seção de choque associada a cada estado final $X$ pode ser escrita como [84]

$$
E^{\prime} \frac{d \sigma_{l N \rightarrow l X}}{d^{3} \vec{k}^{\prime}}=\int \frac{\left[d \Phi_{X}\right]}{32 \pi^{3}\left(s-m_{N}^{2}\right)}(2 \pi)^{4} \delta\left(P+k-k^{\prime}-P_{X}\right)\left\langle\left|\mathcal{M}_{X}\right|^{2}\right\rangle_{\text {spin }},
$$

onde $\left[d \Phi_{X}\right]$ é o elemento invariante do espaço de fase para o estado final $X$ e $\mathcal{M}_{X}$ é a amplitude de transição correspondente.

O rótulo "spin" indica que se deve tomar a média sobre as polarizações de spin no estado inicial e também somar sobre todas as polarizações de spin possíveis no estado final.

A amplitude de transição, $\mathcal{M}_{X}$, pode ser decomposta em um produto de dois termos: um de origem eletromagnética, denotando a interação entre o lépton, digamos, um elétron, e o fóton e outro, dado por um elemento de matriz representando as transições de estado hadrônico,

$$
\mathcal{M}_{X}=\frac{i e}{q^{2}}\left[\bar{u}\left(\vec{k}^{\prime}\right) \gamma^{\mu} u(\vec{k})\right]\left\langle X\left|J_{\mu}(0)\right| N(P)\right\rangle
$$

Nesta equação, $J_{\mu}$ é a corrente de transição hadrônica e $|N(P)\rangle$ indica o estado contendo um nucleon de momento $P$. Quadrando-se a amplitude de transição e rearranjando-se os termos, a seção de choque para esse processo inclusivo pode ser escrita como [84]

$$
E^{\prime} \frac{d \sigma_{e^{-} N}}{d^{3} \vec{k}^{\prime}}=\frac{1}{32 \pi^{3}\left(s-m_{N}^{2}\right)} \frac{e^{2}}{q^{4}} 4 \pi L^{\mu \nu} W_{\mu \nu}
$$


onde $L^{\mu \nu}$ e $W^{\mu \nu}$ são os tensores leptônico e hadrônico, respectivamente.

Trabalhando-se em um regime de altas energias, onde pode-se desprezar a massa do elétron, o tensor leptônico é dado por

$$
\begin{aligned}
L^{\mu \nu} & \equiv\left\langle\bar{u}\left(\vec{k}^{\prime}\right) \gamma^{\mu} u(\vec{k}) \bar{u}(\vec{k}) \gamma^{\nu} u\left(\vec{k}^{\prime}\right)\right\rangle_{\mathrm{spin}} \\
& =2\left(k^{\mu} k^{\prime \nu}+k^{\nu} k^{\prime \mu}-g^{\mu \nu} k \cdot k^{\prime}\right)
\end{aligned}
$$

e o tensor hadrônico é definido como a transformada de Fourier do valor esperado das correntes de transição hadrônica calculadas no estado que representa o nucleon:

$$
\begin{aligned}
4 \pi W_{\mu \nu} \equiv & \sum_{\text {estados } X} \int\left[d \Phi_{X}\right](2 \pi)^{4} \delta\left(P+q-P_{X}\right) \\
& \times\left\langle\left\langle N(P)\left|J_{\nu}^{\dagger}(0)\right| X\right\rangle\left\langle X\left|J_{\mu}(0)\right| N(P)\right\rangle\right\rangle_{\text {spin }} \\
= & \int d^{4} y e^{i q \cdot y}\left\langle\left\langle N(P)\left|J_{\nu}^{\dagger}(y) J_{\mu}(0)\right| N(P)\right\rangle\right\rangle_{\text {spin }} .
\end{aligned}
$$

O tensor hadrônico, $W^{\mu \nu}$, não pode ser calculado via métodos perturbativos já que as correntes de transição hadrônica que se encontram em sua definição não são conhecidas. Sendo assim, a falta de conhecimento sobre $W^{\mu \nu}$ é simplesmente parametrizada da forma mais geral possível. Esta parametrização, no entanto, deve respeitar algumas propriedades já conhecidas das interações hadrônicas:

- Conservação da corrente eletromagnética: $q_{\mu} W^{\mu \nu}=q_{\nu} W^{\mu \nu}=0$.

- Simetria por inversão espacial (paridade) e inversão temporal: $W^{\mu \nu}=W^{\nu \mu}$.

- Conservação da simetria por paridade: $W^{\mu \nu}$ não pode conter nenhum quadrivetor antisimétrico ${ }^{2}$.

O tensor hadrônico pode ser parametrizado em termos das quantidades $g^{\mu \nu}, P^{\mu}$ e $q^{\mu}$ presentes no vértice hadrônico da Figura 1.1 sendo que, após utilizadas as considerações

\footnotetext{
${ }^{2}$ Isto não é verdade quando os processos envolvendo bósons do setor fraco são considerados. Como estes processos não conservam a simetria por paridade, um termo antisimétrico nos índices espaciais se faz necessário na definição de $W^{\mu \nu}$.
} 
acima, sua forma final é dada por

$$
W_{\mu \nu}=W_{1}\left(-g_{\mu \nu}+\frac{q_{\mu} q_{\nu}}{q^{2}}\right)+\frac{W_{2}}{P \cdot q}\left(P_{\mu}-q_{\mu} \frac{P \cdot q}{q^{2}}\right)\left(P_{\nu}-q_{\nu} \frac{P \cdot q}{q^{2}}\right)
$$

onde $W_{1,2}$ são as chamadas "funções de estrutura inelásticas".

Finalmente, calculando-se o produto entre os tensores leptônico e hadrônico e rearranjando-se os termos, a seção de choque inclusiva para o DIS no referencial do laboratório é dada por:

$$
\frac{d \sigma_{e^{-} N}}{d E^{\prime} d \Omega}=\frac{\alpha_{\mathrm{em}}^{2}}{4 m_{N} E^{2} \sin ^{4}(\theta / 2)}\left[2 W_{1}\left(\nu, Q^{2}\right) \sin ^{2} \frac{\theta}{2}+W_{2}\left(\nu, Q^{2}\right) \cos ^{2} \frac{\theta}{2}\right]
$$

onde $d \Omega$ é o elemento de ângulo sólido que identifica a direção do lépton após o espalhamento e $\alpha_{\mathrm{em}}$ é a constante de acoplamento eletromagnético.

Através da medida experimental da dependência com $Q^{2}$ das funções de estrutura inelásticas pode-se, via transformada de Fourier, extrair informações sobre a distribuição espacial dos objetos que constituem a estrutura interna do nucleon alvo. Para a determinação de $W_{1,2}$ é necessário realizar-se uma série de medidas variando-se o ângulo de espalhamento $(\theta)$ e a energia do lépton incidente $(E)$ para cada valor de $\nu$ e $Q^{2}$.

\subsection{O modelo a pártons e a QCD}

Em 1969, supondo que o nucleon fosse composto por férmions puntiformes, que posteriormente foram chamados de "pártons" por Feynman [83] e mais tarde identifi-

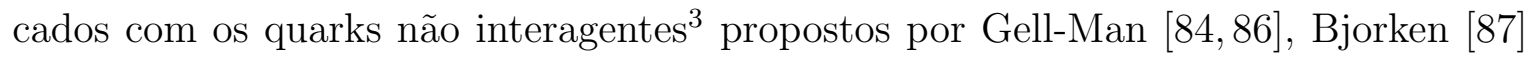
percebeu que, no limite em que o próton se move com um momento longitudinal infinito (o chamado referencial de Breit, $P_{z} \rightarrow \infty$ ), o quadri-momento do párton, $p_{\mu}$,

\footnotetext{
${ }^{3}$ Feynman argumentou que, em uma primeira aproximação, os pártons não deveriam interagir entre eles pois, no limite em que o próton se move com momento infinito, existe uma separação de escalas entre as (lentas) interações párton-párton e a (rápida) interação com o lépton espalhado [85].
} 
seria uma fração do quadri-momento do próton, $P_{\mu}$,

$$
p_{\mu}=x P_{\mu}
$$

e, após a colisão, o momento do párton seria igual a $x P+q$.

Considerando-se que os pártons sejam partículas leves, de modo que suas massas possam ser desprezadas, tem-se

$$
x=\frac{Q^{2}}{2 \nu m_{N}},
$$

que é exatamente uma das variáveis (a variável $x$ de Bjorken) utilizadas na descrição do DIS, como apresentado na seção anterior.

A medida em que $Q^{2}$ aumenta, o fóton começa a "resolver" os pártons dentro do próton. Ou seja, para grandes valores de $Q^{2}$ o espalhamento inelástico entre um elétron e um próton é visto como um espalhamento elástico entre um elétron e um párton constituinte ( $e+q \rightarrow e+q$, onde $q$ denota um párton). A energia no centro de massa do espalhamento entre o elétron e o párton é, nessas condições, igual a

$$
\hat{s}=(x P+k)^{2} \approx 2 x P k \approx x s .
$$

Uma vez que esse processo deve ser idêntico à reação elástica elétron-muon, $e+\mu \rightarrow$ $e+\mu$, cuja seção de choque é [84]

$$
\frac{d \sigma_{e^{-} \mu}}{d E^{\prime} d \Omega}=\frac{\alpha_{\mathrm{em}}^{2}}{4 m_{\mu} E^{2} \sin ^{4}(\theta / 2)}\left[\cos ^{2} \frac{\theta}{2}-\frac{q^{2}}{2 m_{\mu}^{2}} \sin ^{2} \frac{\theta}{2}\right] \delta\left(\nu+\frac{q^{2}}{2 m_{\mu}^{2}}\right),
$$

comparando-se as equações (1.14) e (1.18), é simples se obter as expressões para as funções de estrutura inelástica referentes aos pártons

$$
\begin{gathered}
2 m W_{1}^{\text {parton }}\left(\nu, Q^{2}\right)=\frac{Q^{2}}{2 m \nu} \delta\left(1-\frac{Q^{2}}{2 m \nu}\right) \\
\nu W_{2}^{\text {parton }}\left(\nu, Q^{2}\right)=\delta\left(1-\frac{Q^{2}}{2 m \nu}\right) .
\end{gathered}
$$


Dessa forma as funções de estrutura partônicas dependem somente da variável $x$ e não mais de $\nu$ e $Q^{2}$ separadamente. Esta propriedade foi prevista por Bjorken [88] e ficou conhecida como "scaling de Bjorken". Essa importante propriedade introduz as chamadas funções de estrutura do nucleon, $F_{1}$ e $F_{2}$, através das transformações

$$
\begin{gathered}
W_{1}\left(\nu, Q^{2}\right) \rightarrow F_{1}(x) \equiv m_{N} W_{1}\left(\nu, Q^{2}\right), \\
W_{2}\left(\nu, Q^{2}\right) \rightarrow F_{2}(x) \equiv \nu W_{2}\left(\nu, Q^{2}\right)
\end{gathered}
$$

Ainda em 1969, medidas realizadas no Stanford Linear Accelerator Center (SLAC) confirmaram as previsões de Bjorken [89]. Como pode ser visto na Figura 1.2, as medidas realizadas pelo SLAC indicaram que $F_{2}$ não possuía nenhuma dependência com a variável $Q^{2}$ para $\omega=1 / x=4$. A confirmação da previsão de Bjorken foi interpretada como a evidência de que o próton é composto por partículas elementares puntiformes, os pártons de Feynman. Atualmente, as partículas do modelo proposto por Bjorken são identificadas com os quarks da QCD.

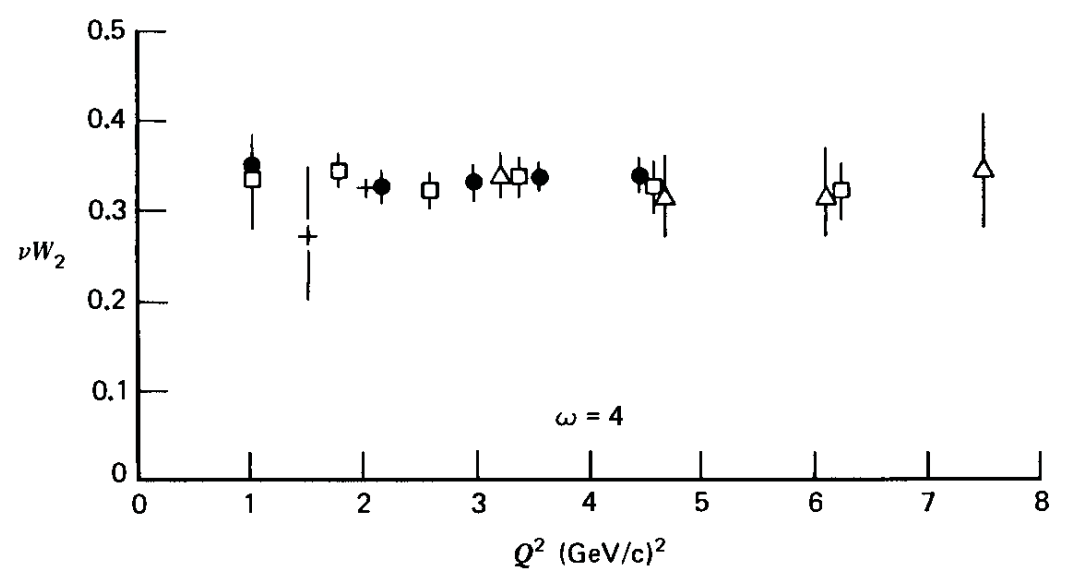

Figura 1.2: Medidas de $F_{2}$ realizadas no SLAC. Extraído de [84].

Ao decompor-se o próton em pártons que se movem livremente em seu interior, a seção de choque elétron-próton passa a ser a soma incoerente da interação do elétron com cada um dos pártons, pesada por uma "densidade de probabilidade", $f_{i}(\xi)$, de se 
encontrar cada párton $q_{i}$ carregando uma fração $\xi$ do momento longitudinal do próton:

$$
\frac{d^{2} \sigma}{d x d Q^{2}}=\sum_{i} \int d \xi f_{i}(\xi) \frac{d^{2} \hat{\sigma}}{d x d Q^{2}}
$$

A equação (1.23) evidencia a hipótese de que a seção de choque do DIS pode ser fatorizada numa interação de curta distância, descrita pela seção de choque a nível partônico, $\frac{d^{2} \hat{\sigma}}{d x d Q^{2}}$, e uma função que contém os efeitos de longa distância, a "função de distribuição de pártons", $f_{i}(\xi)$.

Assumindo-se que os pártons são férmions de Dirac de spin $1 / 2$ e que carregam uma fração $\xi$ do momento do próton, o seguinte resultado é encontrado [90],

$$
F_{2}(x)=2 x F_{1}(x)=\sum_{i} e_{i}^{2} \int_{0}^{1} d \xi \delta(x-\xi) \xi f_{i}(\xi)=\sum_{i} e_{i}^{2} x f_{i}(x)
$$

onde $e_{i}$ é a carga elétrica do párton de sabor $i$.

A equação (1.24) é conhecida como "relação de Callan-Gross" e é válida somente nas regiões onde o scaling de Bjorken é válido. Além disso, tem-se que a fração de momento do nucleon carregada pelo párton é igual a variável $x$ de Bjorken.

A partir da relação de Callan-Gross vê-se que a função de estrutura $F_{2}(x)$ mede a distribuição de momento fracionário dos pártons dentro do próton, a menos de um fator multiplicativo. Devido a este fato, a equação (1.24) é o resultado mais importante do modelo a pártons.

As PDFs fornecem a informação sobre o conteúdo partônico existente nos hádrons colidentes a depender, por enquanto, da fração de momento provada ${ }^{4}$ na colisão. Todavia, os pártons não são observados livremente na natureza uma vez que estão sempre confinados nos hádrons, partículas estas que são medidas pelos detectores experimentais. Sendo assim, como os pártons não são observados diretamente a nível experimental, não temos acesso direto à distribuição de pártons nos hádrons e, portanto, as PDFs não podem ser totalmente determinadas via pQCD. Por esse motivo, como

\footnotetext{
$Q$.

${ }^{4}$ Nas próximas seções mostra-se que as PDFs também são dependentes da virtualidade da colisão,
} 
mencionado anteriormente, as PDFs dão conta de efeitos de longa distância, isto é, de caráter não perturbativo.

Embora a pQCD não seja capaz de fornecer os valores iniciais para as PDFs, ela é capaz de prever a sua evolução com as variáveis $x$ e/ou $Q^{2}$, como veremos nas próximas seções. Sendo assim, assume-se uma parametrização analítica para as PDFs em uma escala de momento arbitrária (mas perturbativa) $Q_{0}$ que é utilizada como condição inicial. A determinação de cada $f_{i}(x)$ se dá, então, através da análise de uma grande quantidade de dados experimentais ${ }^{5}$. Estas funções devem satisfazer certas regras de soma de modo a fixar o número de pártons do tipo $i(i=u, d, s \ldots)$ que constituem um nucleon $[84,93]$. Outra regra de soma importante diz respeito ao momento carregado pelos pártons que compõem o nucleon: a soma da fração de momento carregada por cada um dos pártons constituintes deve resultar no momento total do nucleon.

A partir dos dados experimentais medidos pela colaboração entre o SLAC e o Massachusetts Institute of Technology [94] encontrou-se que os pártons (e antipártons) carregam aproximadamente metade do momento total do nucleon, a outra metade devendo ser carregada por partículas que não deveriam interagir nem eletromagnéticamente e nem fracamente. Estas partículas posteriormente foram identificadas como os gluons, os quanta da interação forte [86] e foi o ponto de partida da QCD, a teoria de campo dessas interações.

A descrição fornecida pela QCD para as interações hadrônicas é baseada em um modelo de partículas elementares - os quarks - dotados de "carga de cor" e que interagem por troca de campos de gauge - os gluons - que são dotados de uma carga de cor e outra de anticor, porém nunca formando um estado de cor "branca", ou seja, um estado de cor nula.

A QCD é uma teoria de campos com simetria de gauge local $S U(3)$, correspondendo, portanto, a três possíveis cores (com suas respectivas anticores), $N_{c}=3$. O campo dos quarks, que possuem spin $1 / 2$, é representado por espinores, $q_{a}$, onde $a=1,2,3\left(=N_{c}\right)$ é o índice de cor (o índice espinorial foi omitido). O campo dos

\footnotetext{
${ }^{5}$ Maiores detalhes sobre como as PDFs são obtidas podem ser encontrados em [91, 92].
} 
bósons de gauge da teoria, os gluons que possuem spin 1, é representado por $A^{\mu} \equiv A_{a}^{\mu} t_{a}$, $\mu$ sendo o índice de Lorentz, $a=1, \ldots, 8\left(N_{c}^{2}-1\right)$ e $t^{a}$ representando os geradores do grupo $S U(3)$, matrizes hermitianas, de traço nulo e de dimensão $N_{c} \times N_{c}$.

A não ser pela simetria sob o grupo de gauge $S U(3)$ (ao invés do grupo $U(1)$ ), a lagrangeana da QCD é a mesma que a da Eletrodinâmica Quântica (Quantum Elctrodynamics - QED) com o campo dos quarks (antiquarks) correspondendo aos elétrons (pósitrons) e o campo dos gluons correspondendo aos fótons [84,93]:

$$
\mathcal{L}=\sum_{f} \bar{q}_{a}^{f}\left(i \gamma_{\mu} D^{\mu}-m\right)_{a b} q_{b}^{f}-\frac{1}{4} F_{\mu \nu} F^{\mu \nu},
$$

onde $\gamma^{\mu}$ representam as matrizes de Dirac,

$$
D^{\mu}=\partial^{\mu}-i g A^{\mu}
$$

representa a derivada covariante, $m$ representa a massa do quark de sabor $f$ e $g$ representa o acoplamento forte, que expressa a intensidade das cargas de cor.

O tensor de campo gluônico, $F^{\mu \nu}$, é dado por

$$
F^{\mu \nu}=F_{a}^{\mu \nu} t_{a}=\partial^{\mu} A^{\nu}-\partial^{\nu} A^{\mu}-i g\left[A^{\mu}, A^{\nu}\right]
$$

Para teorias abelianas, como a QED, o comutador na equação acima se anula. Já para uma teoria não-abeliana, como a $\mathrm{QCD}$, tem-se que $\left[A^{\mu}, A^{\nu}\right]=A_{a}^{\mu} A_{b}^{\nu}\left[t_{a}, t_{b}\right]$, com $\left[t_{a}, t_{b}\right]=i f_{a b c} t_{c}$, sendo $f_{a b c}$ as constantes de estrutura do grupo $S U(3)$, que são antissimétricas pela troca de dois índices de cor.

O fato do grupo de simetria da QCD ser não-abeliano é o motivo da maior diferença entre ambas as teorias: enquanto na QED não existem interações entre fótons, pois estes possuem carga elétrica nula, na QCD, onde as interações ocorrem sempre entre parículas com carga de cor não nula, os gluons podem interagir entre si. Este fato pode ser visto através do último termo da equação (1.27).

Conforme mencionado anteriormente, a QCD é uma teoria de campos com simetria 
de gauge local $S U(3)$. Neste caso, a lagrangeana definida na equação (1.25) é invariante sob as seguintes transformações de gauge:

$$
\begin{gathered}
\psi(x) \rightarrow \psi^{\prime}(x)=U(x) \psi(x) \\
\bar{\psi}(x) \rightarrow \bar{\psi}^{\prime}(x)=\psi(x) U^{-1}(x) \\
A_{\mu}(x) \rightarrow A_{\mu}^{\prime}(x)=U(x) A_{\mu}(x) U^{-1}(x)-\frac{i}{g}\left(\partial_{\mu} U(x)\right) U^{-1}(x)
\end{gathered}
$$

onde $U(x)=e^{i t_{a} \theta_{a}(x)}$, com $\theta_{a}(x)$ sendo um conjunto de funções reais dependentes da posição e do tempo. Aplicando-se as transformações listadas acima na equação (1.25), pode-se mostrar que $\mathcal{L}\left(\psi^{\prime}, \bar{\psi}^{\prime}, A_{\mu}^{\prime}\right)=\mathcal{L}\left(\psi, \bar{\psi}, A_{\mu}\right)$, indicando que, de fato, a lagrangeana da QCD é invariante por tais transformações.

Todavia, a lagrangeana dada pela equação (1.25) representa apenas a parte clássica da teoria. Para uma descrição a nível quântico dos graus de liberdade bosônicos se faz necessária a adição de um termo que fixe o gauge na equação (1.25). Sem tal termo, a equação de movimento para o campo bosônico não pode ser invertida. Isso se deve à arbitrariedade do campo $A_{\mu}$ que, por sua vez, é consequência justamente da liberdade de escolha do gauge na equação (1.30). A fixação do gauge na lagrangeana da QCD permite a definição de um propagador para o campo dos gluons e a quantização dos campos $A_{\mu}{ }^{6}$

Por fim, caso o termo que fixa o gauge na equação (1.25) seja escrito na forma covariante, $\mathcal{L}_{\text {fix }} \propto\left(\partial^{\mu} A_{\mu}\right)^{2}$, deve-se adicionar ainda um outro termo, os chamados campos "fantasmas" de Fadeev-Popov, $\mathcal{L}_{F P}$, responsáveis por eliminar os graus de liberdade não físicos gerados a partir da forma covariante do termo $\mathcal{L}_{\text {fix }}$. A forma e mesmo a presença de $\mathcal{L}_{F P}$ é dependente da escolha de gauge. Caso opte-se por trabalhar no gauge axial, $A_{3}=0$, o termo $\mathcal{L}_{F P}$ não se faz necessário, por exemplo.

Como será visto na próxima seção, uma característica importante da interação

\footnotetext{
${ }^{6}$ Embora a simetria de gauge deva ser quebrada explicitamente para que seja possível quantizar os campos bosônicos, esta simetria ainda se faz presente no fato de que qualquer observável físico deve ser independente do gauge escolhido para a construção do propagador dos gluons.
} 
forte é que a constante de acoplamento (forte), $\alpha_{s}$, torna-se muito pequena $\left(\alpha_{s} \ll 1\right)$ no limite de pequenas distâncias, ou equivalentemente, no limite de altas energias ou momentos transferidos. Esta propriedade é chamada de "liberdade assintótica" [11-13] e é o que permite a aplicabilidade da pQCD como ferramenta de cálculo.

A distâncias maiores (ou seja, a energias menores) há um aumento da intensidade da interação entre os quarks e os gluons a ponto de confiná-los no interior dos hádrons, isto é, excluindo-se a possibilidade de se observar quarks e gluons isoladamente. Esta propriedade é conhecida como "confinamento" [14].

O modelo a pártons tratado até aqui ignora completamente o papel dinâmico dos gluons como intermediadores da força forte presente nas interações com os quarks. Essa aproximação despreza a possibilidade de que os quarks possam emitir gluons antes ou depois de serem espalhados pelo fóton virtual e também a possibilidade da produção de pares quark-antiquark $(q \bar{q})$ pelos gluons. A nível de diagramas de Feynman, os processos da QCD que contribuem para a seção de choque do DIS $(e p \rightarrow e X)$ são de ordem $\mathcal{O}\left(\alpha_{e m} \alpha_{s}\right)$, enquanto que os processos do modelo a pártons de Bjorken são de ordem $\mathcal{O}\left(\alpha_{e m}\right)$, o que indica que a possível emissão de gluons deva ser considerada.

\subsubsection{A constante de acoplamento da QCD}

Os processos elementares, isto é, de mais baixa ordem em teoria perturbativa da QCD, são de ordem $\mathcal{O}\left(\alpha_{s}^{2}\right)$. Ao calcularmos o termo seguinte dessa série, o que corresponderia a todos os diagramas de ordem $\mathcal{O}\left(\alpha_{s}^{4}\right)$, conhecidos como diagramas de 1-loop, encontramos divergências que necessitam ser regularizadas de tal forma a se tornarem finitas.

As divergências oriundas do cálculo desses diagramas são de cunho ultravioleta, isto é, ocorrem na região de grandes momentos. Felizmente, divergências deste tipo podem ser removidas sendo absorvidas em redefinições de determinadas quantidades físicas, através de um procedimento denominado "renormalização", que trataremos a 
seguir. No entanto, ao fazer uso deste processo, introduz-se naturalmente uma nova escala na teoria, conhecida como "escala de renormalização", $\mu$, cujo valor é arbitrário, fazendo com que as quantidades renormalizadas dependam explicitamente dessa nova escala.

Uma vez que quantidades físicas mensuráveis não podem depender dessa escala, a constante de acoplamento forte, $\alpha_{s}$, deve ser independente da escolha do valor de $\mu$ (escala em que as subtrações das divergências ultravioletas são realizadas) e, portanto, uma mudança na escala de renormalização deve ser compensada por uma mudança na constante de acoplamento efetiva calculada no ponto de subtração $\mu$ :

$$
\alpha_{s} \equiv \alpha_{s}\left(\mu^{2}\right)=\frac{g^{2}}{4 \pi}
$$

A forma como essa compensação é realizada é regida pela "equação do grupo de renormalização" da QCD. Para estudar a forma como ocorrem as alterações na constante de acoplamento considera-se um observável $R$, sem dimensões e que envolva apenas uma escala, identificada aqui como $Q^{2}$. O fato de $R$ não depender de $\mu$ implica que sua derivada total com respeito a essa variável deve ser nula:

$$
\mu^{2} \frac{d}{d \mu^{2}} R\left(Q^{2} / \mu^{2}, \alpha_{s}\right)=\left[\mu^{2} \frac{\partial}{\partial \mu^{2}}+\mu^{2} \frac{\partial \alpha_{s}}{\partial \mu^{2}} \frac{\partial}{\partial \alpha_{s}}\right] R=0
$$

Definindo-se $t=\ln \left(Q^{2} / \mu^{2}\right)$ e a chamada "função beta da QCD",

$$
\beta\left(\alpha_{s}\right)=\mu^{2} \frac{\partial \alpha_{s}}{\partial \mu^{2}}
$$

a equação (1.32) pode ser reescrita da seguinte forma

$$
\left[-\frac{\partial}{\partial t}+\beta\left(\alpha_{s}\right) \frac{\partial}{\partial \alpha_{s}}\right] R\left(e^{t}, \alpha_{s}\right)=0
$$

A equação (1.34) determina como uma mudança no ponto de subtração $\mu$ é com- 
pensada por uma mudança no acoplamento $\alpha_{s}\left(\mu^{2}\right)$. Introduzindo-se uma constante de acoplamento efetiva, $\alpha_{s}^{\text {eff }}$, que é solução da equação

$$
\frac{d \alpha_{s}^{\mathrm{eff}}\left(t, \alpha_{s}\right)}{d t}=\beta\left(\alpha_{s}\right)
$$

$\operatorname{com} \alpha_{s}=\alpha_{s}^{\text {eff }}\left(t=0, \alpha_{s}\right)$, tem-se que

$$
t=\int_{\alpha_{s}}^{\alpha_{s}^{\mathrm{eff}}\left(t, \alpha_{s}\right)} \frac{d \alpha}{\beta(\alpha)}
$$

A nível de diagramas de 1-loop, a função $\beta$ da QCD é dada por [95]

$$
\beta\left(\alpha_{s}\right)=-\beta_{0} \alpha_{s}^{2} \quad \text { com } \quad \beta_{0}=\frac{11 C_{A}-4 n_{f} T_{R}}{12 \pi},
$$

onde $C_{A}=3, T_{R}=1 / 2$ e $n_{f}$ é o número de sabores considerados.

As quantidades $C_{A}$ e $T_{R}$ estão relacionadas às matrizes de cor do grupo $S U(3)$ da álgebra de Lie, as matrizes de Gell-Man, e ainda aos geradores da representação fundamental e constantes de estrutura deste grupo [96].

Usando-se a equação (1.37) em (1.36) obtém-se

$$
\alpha_{s}\left(\mu^{2}\right)=\frac{\alpha_{s}\left(\mu_{0}^{2}\right)}{1+\beta_{0} \alpha_{s}\left(\mu_{0}^{2}\right) \ln \left(\mu^{2} / \mu_{0}^{2}\right)}
$$

que especifica $\alpha_{s}$ a uma escala $\mu$, a partir dessa mesma quantidade, numa escala inicial (conhecida) $\mu_{0}$.

Escolhendo-se $\mu^{2}=Q^{2}$, vê-se que o valor da constante de acoplamento diminui com o crescimento da escala $Q^{2}$. Isto se deve ao valor negativo da função $\beta$. O fato da QCD se tornar uma "teoria livre" $\left(\alpha_{s} \rightarrow 0\right)$ no limite de grandes valores de $Q^{2}$, propriedade denominada "liberdade assintótica" [11-13], como dito anteriormente, e é o que permite a aplicação da teoria perturbativa. No sentido contrário, para baixos valores da escala $Q^{2}$, a constante de acoplamento se torna grande $\left(\alpha_{s}>1\right)$ impossibilitando a convergência da expansão perturbativa e consequentemente invalidando 
todos os cálculos e previsões realizadas utilizando-se pQCD. Nesses casos faz-se uso dos chamados "métodos não perturbativos", como as regras de soma da QCD [97] e QCD na rede $[98,99]$ para o estudo de observáveis físicos como massas e larguras de decaimento.

Como veremos a seguir, com intuito de contornar esse obstáculo e garantir a aplicabilidade da pQCD, podemos introduzir a quantidade $\Lambda_{Q C D}$, um parâmetro a ser fixado através de descrições globais de dados experimentais, dado por

$$
\Lambda_{Q C D}^{2}=\mu_{0}^{2} \exp \left(-\frac{1}{\beta_{0} \alpha_{s}\left(\mu_{0}^{2}\right)}\right)
$$

para exprimir $\alpha_{s}\left(\mu^{2}\right)$ em termos de uma escala geral, sem fazer nenhuma referência à escala $\mu_{0}^{2}$ :

$$
\alpha_{s}\left(Q^{2}\right)=\frac{1}{\beta_{0} \ln \left(Q^{2} / \Lambda_{Q C D}^{2}\right)} .
$$

Pela equação acima observa-se que quando $Q^{2}$ for da ordem de $\Lambda_{Q C D}^{2}$, tem-se que $\alpha_{s} \sim 1$. Conforme mencionado acima, nessas condições, a teoria perturbativa deixa de ser válida e aproxima-se cada vez mais do regime não perturbativo da QCD. Por esse motivo, $\Lambda_{Q C D}$ é conhecida como a escala não perturbativa da QCD.

\subsection{As equações de evolução da QCD}

Ao contribuírem para a seção de choque do DIS, os diagramas de emissão de gluons e de produção de pares também contribuem para a função de estrutura do próton, $F_{2}$. A inclusão desses diagramas modifica a função de estrutura do próton prevista pelo modelo a pártons (vide equação (1.24)) incluindo um termo proporcional a $\alpha_{s}\left(Q^{2}\right) \ln \left(Q^{2} / \Lambda_{Q C D}\right)$ [84]. Isso faz com que as distribuições de pártons passem a depender explicitamente da virtualidade do fóton, causando a violação do scaling de Bjorken e invalidando a relação de Callan-Gross.

Embora não seja possível obter os valores iniciais para cada distribuição de párton 
existente via pQCD é possível determinar como essas quantidades se modificam a medida em que a escala $Q^{2}$ vai aumentando. A evolução das distribuições de pártons com respeito à variável $Q^{2}$ é descrita pelas equações do grupo de renormalização da QCD, as equações DGLAP [15-18]. Portanto estas equações fornecem o quão pronunciada é a influência das correções da pQCD nas funções de distribuição de pártons com $Q^{2}$ e, consequentemente, o quão forte é a violação do scaling de Bjorken na função de estrutura $F_{2}$.

Antes de apresentar as equações DGLAP um comentário importante deve ser feito. Cálculos perturbativos se baseiam em uma expansão em série onde o parâmetro de expansão, $\alpha_{s}\left(Q^{2}\right)$ para a $\mathrm{QCD}$, é pequeno $(\ll 1)$ fazendo com que a série convirja a um resultado finito (e para a qual termos de mais alta ordem são suprimidos). É importante notar que, embora $\alpha_{s}\left(Q^{2}\right) \ll 1$, temos que $\ln \left(Q^{2} / \Lambda_{Q C D}\right) \gg 1$ nos diagramas de emissão de pártons a medida em que $Q^{2}$ aumenta. Sendo assim, o parâmetro da expansão passa a ser $\alpha_{s}\left(Q^{2}\right) \ln \left(Q^{2} / \Lambda_{Q C D}\right) \sim 1$ e, consequentemente, a série perturbativa seria divergente. As equações DGLAP fazem a "ressoma" 7 dessas divergências e fornecem um resultado finito.

Finalmente, as equações DGLAP para as distribuições de um quark $f_{i}\left(x, Q^{2}\right) \equiv$ $q_{i}\left(x, Q^{2}\right)$ de sabor $i$ e de gluons, $g\left(x, Q^{2}\right)$, dentro de um próton obedecem $[15-18,84$, $93,101,102]$,

$$
\begin{aligned}
& \frac{\partial q_{i}\left(x, Q^{2}\right)}{\partial \ln Q^{2}}=\frac{\alpha_{s}}{2 \pi}\left[\int_{x}^{1} \frac{d x_{1}}{x_{1}}\left(P_{q q}\left(\frac{x}{x_{1}}\right) q_{i}\left(x_{1}, Q^{2}\right)+P_{q g}\left(\frac{x}{x_{1}}\right) g\left(x_{1}, Q^{2}\right)\right)\right], \\
& \frac{\partial g\left(x, Q^{2}\right)}{\partial \ln Q^{2}}=\frac{\alpha_{s}}{2 \pi}\left[\int_{x}^{1} \frac{d x_{1}}{x_{1}}\left(P_{g q}\left(\frac{x}{x_{1}}\right) q_{S}\left(x_{1}, Q^{2}\right)+P_{g g}\left(\frac{x}{x_{1}}\right) g\left(x_{1}, Q^{2}\right)\right)\right],
\end{aligned}
$$

(a equação DGLAP para a distribuição de antiquarks é análoga à equação (1.41), porém com a troca $\left.q_{i}\left(x, Q^{2}\right) \rightarrow \bar{q}_{i}\left(x, Q^{2}\right)\right)$ onde $q_{S}\left(x, Q^{2}\right)$ é a chamada "distribuição

\footnotetext{
${ }^{7}$ Para maiores detalhes sobre métodos de ressoma veja [100].
} 
singleta de quark",

$$
q_{S}\left(x, Q^{2}\right) \equiv \sum_{i}\left[q_{i}\left(x, Q^{2}\right)+\bar{q}_{i}\left(x, Q^{2}\right)\right]
$$

As funções $P_{i j}(i, j=q q, g q, q g, g g)$ que figuram nas equações DGLAP são denominadas "funções de splitting". Estas funções fornecem a probabilidade de um párton $i$ emitir um párton $j$ e, em ordem dominante (Leading Order - LO) em $\alpha_{s}$, são dadas por $[15-18,84,101,102]$

$$
\begin{gathered}
P_{q q}(z)=C_{F}\left[\frac{1+z^{2}}{\left(1-z^{2}\right)_{+}}+\frac{3}{2} \delta(1-z)\right] \\
P_{q g}(z)=\frac{1}{2}\left[z^{2}+\left(1-z^{2}\right)\right] \\
P_{g q}(z)=C_{F}\left[\frac{1+(1-z)^{2}}{z}\right] \\
P_{g g}(z)=2 C_{A}\left[\frac{z}{\left(1-z^{2}\right)_{+}}+\frac{1-z}{z}+z(1-z)\right]+\frac{11 C_{A}-2 n_{f}}{6} \delta(1-z),
\end{gathered}
$$

onde os fatores de cor, $C_{F}$ e $C_{A}$, são dados por $C_{F}=\frac{N_{C}^{2}-1}{2 N_{C}}, C_{A}=N_{C}$ e $n_{f}$ representa o número de sabores de quarks considerado (aqui identifica-se $N_{C}$ como o número de cores da teoria, $\left.N_{C}^{Q C D}=3\right)$ e $z=x / x_{1}$.

As distribuições "+" são dadas pela prescrição:

$$
\int_{0}^{1} d x \frac{f(x)}{1+x}=\int_{0}^{1} d x \frac{f(x)-f(1)}{1-x}
$$

A primeira equação DGLAP, a equação (1.41), expressa o fato de que o quark com fração de momento $x$ no lado esquerdo dessa equação pode ter vindo de um quark com fração de momento $x_{1}$ que emitiu um gluon ou de um gluon que emitiu um par quark-antiquark. A probabilidade do primeiro processo ocorrer é proporcional a $\alpha_{s} P_{q q}$ e, para o segundo processo, $\alpha_{s} P_{q g}$. A interpretação é semelhante para as demais equações DGLAP. Por envolverem as distribuições partônicas elevadas apenas à 
primeira potência, diz-se que as equações DGLAP são equações de evolução "lineares".

Uma vez que as distribuições de pártons fornecem a densidade de pártons, que são provadas com resolução $1 / Q$ (onde $Q$ é a virtualidade da sonda que prova o meio partônico numa colisão, um fóton no caso do DIS, por exemplo), elas "contêm" todos os pártons que possuem momento transversal menor que $Q$ mas não pártons com momento transversal maior, já que estes não podem ser provados. Sendo assim, a distribuição de gluons $x g\left(x, Q_{0}^{2}\right)$, que é evoluída para uma escala $Q^{2}$, contém todos os gluons que possuem momento transversal $k_{T}^{2} \leq Q^{2}$, ou seja,

$$
x g\left(x, Q^{2}\right) \equiv \int^{Q^{2}} d k_{T}^{2} \hat{g}\left(x, k_{T}^{2}\right)
$$

onde $\hat{g}\left(x, k_{T}^{2}\right)$ é a distribuição não integrada de gluons.

Conforme apontado anteriormente, a pQCD permite determinar somente a evolução das distribuições partônicas através das equações DGLAP. As condições iniciais para a evolução são, por outro lado, de natureza não perturbativa, devendo ser extraídas a partir de experimentos globais para uma dada virtualidade do fóton, $Q^{2}$. Assumindose que as distribuições partônicas são universais, isto é são independentes do processo considerado, após determiná-las em um certo valor de referência, $Q_{0}^{2}$, pode-se, com o auxílio das equações DGLAP, determinar seus valores para outras virtualidades e assim utilizá-las no cálculos de processos de interesse.

Após o colisor elétron-próton HERA iniciar suas atividades verificou-se, através de medidas da função de estrutura do próton, $F_{2}^{p}$ [22-32], que o scaling de Bjorken era, de fato, violado. Nas regiões de pequenos valores de $x$ a função de estrutura do próton deixa de ser constante e cresce com o aumento de $Q^{2}$. Esse fato significa que o próton parece ser "diferente" dependendo da maneira com que olhamos para ele: na região onde $F_{2}^{p} \sim$ constante, apenas os quarks de valência são observados; a medida que valores menores de $x$ são acessados $F_{2}^{p}$ cresce com $Q^{2}$, significando que um número maior de pártons estão sendo resolvidos dentro do próton. Este fato deu início a muitas pesquisas, principalmente na região de pequenos valores de $x$. Como 
veremos na próxima seção, o crescimento de $F_{2}^{p}$ se deve principalmente ao aumento da densidade de gluons dentro do próton.

Como mostra a Figura 1.3, a região de $Q^{2}$ e $x$ grandes os dados experimentais para a função de estrutura do próton são muito bem descritos fazendo-se uso das equações de evolução DGLAP. No entanto, a descrição segundo as DGLAP não é capaz de descrever os dados experimentais na região de pequenos valores de $x$ e $Q^{2}$ mesmo após a inclusão de contribuições advindas de processos de mais alta ordem em pQCD. O motivo para tal se deve ao fato de que a região de $Q^{2} \lesssim 1 \mathrm{GeV}^{2}$ é não perturbativa e, portanto, está fora do limite de aplicabilidade destas equações.

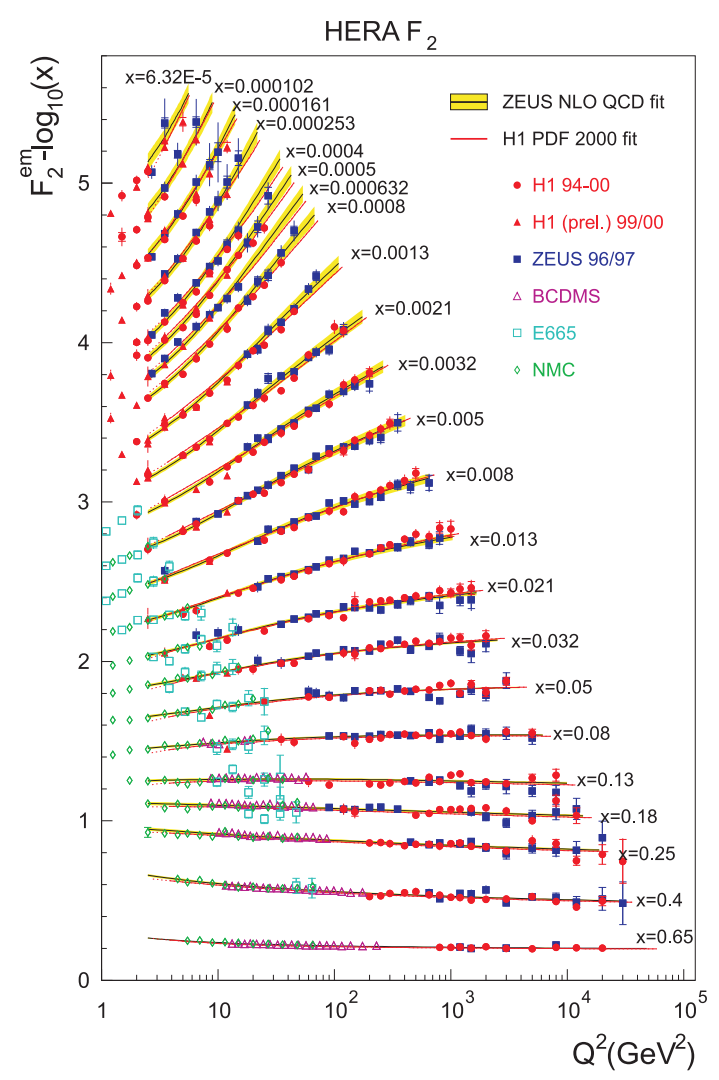

Figura 1.3: Dados experimentais da função de estrutura do próton, $F_{2}$ comprovando a violação do scaling de Bjorken. Extraído de [103].

Atualmente existem na literatura diversos grupos que se dedicam a obter parametrizações para as distribuições partônicas, isto é, soluções das equações de DGLAP para 
todos os tipos de pártons, incorporando paulatinamente os novos dados experimentais em descrições globais.

As parametrizações CTEQ6L [92], GRV98 [104] e MRST01LO [105] foram utilizadas nesta tese. É importante notar que essas, assim como outras distribuições da literatura, evoluem com $Q^{2}$ segundo às equações de evolução DGLAP, o que faz com que cresçam fortemente para pequenos valores de $x$ e grandes valores de $Q^{2}$, como veremos a seguir.

\subsubsection{As equações DGLAP no limite de $x \rightarrow 0$ e $Q^{2} \rightarrow \infty$}

Nas subseções anteriores encontramos que, a partir de um certo valor para $Q^{2}$, o fóton trocado no processo de DIS começa a ter resolução $(\sim 1 / Q)$ suficiente para provar os quarks e gluons que constituem o próton. Outro resultado importante é o fato de que se os quarks não interagissem entre si nenhuma estrutura interna adicional, isto é nada além dos quarks de valência, deveria ser encontrada a medida que o valor de $Q^{2}$ aumentasse. Neste caso o scaling de Bjorken seria exato e o modelo a pártons seria satisfatório.

No entanto, as equações DGLAP conduzem a um crescimento exacerbado das distribuições de pártons com o aumento de $Q^{2}$ e indicam que o scaling de Bjorken é violado, como mostra a Figura 1.3.

Assim, é natural pensar que, se o número de pártons que dividem o momento do próton aumenta com $Q^{2}$, existe uma possibilidade, também crescente, de se encontrar um párton carregando uma fração de momento, $x$, muito pequena e uma probabilidade decrescente de se encontrar pártons com altos valores de $x$, uma vez que esses pártons estariam perdendo momento ao irradiar gluons e formando, por conseguinte, um meio de alta densidade partônica. É interessante, então, analisar qual é a previsão das equações de evolução da QCD no limite em que $x \rightarrow 0$ e $Q^{2} \rightarrow \infty$.

Para isso, consideremos as equações DGLAP apresentadas na seção anterior. A 
partir da análise das funções de splitting, $P_{i j}$, verifica-se que as funções associadas ao setor de gluons, $P_{g g}(z)$ e $P_{g q}(z)$, são singulares para $z \rightarrow 0$ e/ou $x \rightarrow 0$, pois, como definido anteriormente, $z=x / x_{1}$.

As demais funções de splitting, associadas ao setor de quarks, são regulares neste limite e, consequentemente, o comportamento das distribuições partônicas em pequenos valores de $x$ é determinado, predominantemente, pela dinâmica gluônica.

Sendo assim, pode-se aproximar a equação DGLAP para a distribuição de gluons na região de pequenos valores de $x$ pelo seu termo singular

$$
\frac{\partial g\left(x, Q^{2}\right)}{\partial \ln Q^{2}}=\frac{\alpha_{s}}{2 \pi} \int_{x}^{1} \frac{d x_{1}}{x_{1}} \frac{2 C_{A}}{z} g\left(x_{1}, Q^{2}\right)
$$

onde $P_{g g}(z \rightarrow 0)=2 C_{A} / z$, conforme a equação (1.47), representa o termo singular da função de splitting.

Portanto

$$
x g\left(x, Q^{2}\right)=\frac{\alpha_{s} N_{c}}{\pi} \int_{x}^{1} \frac{d x_{1}}{x_{1}} \int_{Q_{0}^{2}}^{Q^{2}} d \ln Q^{\prime 2} x_{1} g\left(x_{1}, Q^{\prime 2}\right),
$$

onde introduziu-se um corte $\left(Q_{0}^{2}\right)$ na integração sobre as virtualidades do fóton para separar a contribuição não perturbativa (região onde os cálculos efetuados não são mais válidos).

Supondo uma distribuição constante de gluons na região de pequenos valores de $Q^{2}$, isto é, $x g\left(x, Q^{\prime 2}\right) \approx x g_{0}(x)=C$, tem-se

$$
x g_{1}\left(x, Q^{2}\right)=C \frac{3 \alpha_{s}}{\pi} \ln \left(\frac{Q^{2}}{Q_{0}^{2}}\right) \ln \left(\frac{1}{x}\right)
$$

onde $x g_{1}\left(x, Q^{2}\right)$ representa a distribuição de gluons levando-se em consideração a emissão de um gluon.

Para se calcular a contribuição da emissão de dois gluons utiliza-se um processo de iteração, isto é, usa-se o resultado da obtido para a emissão de um gluon como condição inicial para se obter $x g_{2}\left(x, Q^{2}\right)$, e assim sucessivamente. Partindo da equação (1.50) 
com $x_{1} g\left(x_{1}, Q^{2}\right)$ tem-se $(1.52)$

$$
x g_{2}\left(x, Q^{2}\right)=C \frac{1}{2} \frac{1}{2}\left[\frac{3 \alpha_{s}}{\pi} \ln \left(\frac{Q^{2}}{Q_{0}^{2}}\right) \ln \left(\frac{1}{x}\right)\right]^{2} .
$$

Repetindo-se este processo, a distribuição de gluons após a emissão de $n$ gluons é

$$
x g_{n}\left(x, Q^{2}\right)=C \frac{1}{n !} \frac{1}{n !}\left[\frac{3 \alpha_{s}}{\pi} \ln \left(\frac{Q^{2}}{Q_{0}^{2}}\right) \ln \left(\frac{1}{x}\right)\right]^{n}
$$

Somando-se sobre o número de gluons emitidos, $n$, tem-se

$$
\begin{aligned}
x g\left(x, Q^{2}\right) & =C \sum_{n} \frac{1}{n !} \frac{1}{n !}\left[\frac{3 \alpha_{s}}{\pi} \ln \left(\frac{Q^{2}}{Q_{0}^{2}}\right) \ln \left(\frac{1}{x}\right)\right]^{n} \\
& \approx C \exp \left[2 \sqrt{\frac{3 \alpha_{s}}{\pi} \ln \left(\frac{Q^{2}}{Q_{0}^{2}}\right) \ln \left(\frac{1}{x}\right)}\right] .
\end{aligned}
$$

Este resultado é conhecido como "aproximação de duplo logarítmo dominante" (Double Leading Logarithm Approximation - DLLA) e possui consequências interessantes de serem analisadas.

No regime de DLLA, onde $x \rightarrow 0$ e $Q^{2} \rightarrow \infty$, as equações DGLAP conduzem a um crescimento ilimitado da densidade de gluons com o aumento de $Q^{2}$ e a diminuição de $x$. Embora os limites em $Q^{2}$ e $x$, individualmente, levem ao mesmo resultado, existem consequências diferentes para cada um deles em relação à densidade do meio partônico que é formado durante a colisão.

Fixando-se $x$ em um valor não muito pequeno e realizando-se a evolução em $Q^{2}$, a densidade de pártons aumenta à medida em que $Q^{2}$ cresce. No entanto, pelo princípio da incerteza, a área transversal ocupada por cada um desses novos pártons que passam a ser resolvidos decresce com $\sim 1 / Q^{2}$. Então, mesmo com a densidade de pártons aumentando, o sistema partônico produzido pela evolução DGLAP é, efetivamente, cada vez mais diluído, com os pártons acoplados mais fracamente, tornando-se cada vez mais confiável a aplicação da pQCD.

Fazendo-se o procedimento inverso, ou seja, fixando-se $Q^{2}$ em um valor moderado 
e evoluindo a distribuição na variável $x$, a densidade de pártons também aumenta, porém com a área transversal ocupada por cada párton não sendo afetada. Ou seja, a medida que $x \rightarrow 0$ o processo de emissão de gluons se encarrega de aumentar a densidade do meio partônico até um certo ponto, por hora indeterminado, sem alterar a área ocupada por cada párton resolvido. Embora, nos dois casos, a distribuição de gluons aumente indefinidamente para o mesmo valor de energia, $\sqrt{s}$, na segunda situação, forma-se um meio altamente denso, enquanto que no primeiro o meio é mais diluído. A Figura 1.4 ilustra essa discussão.

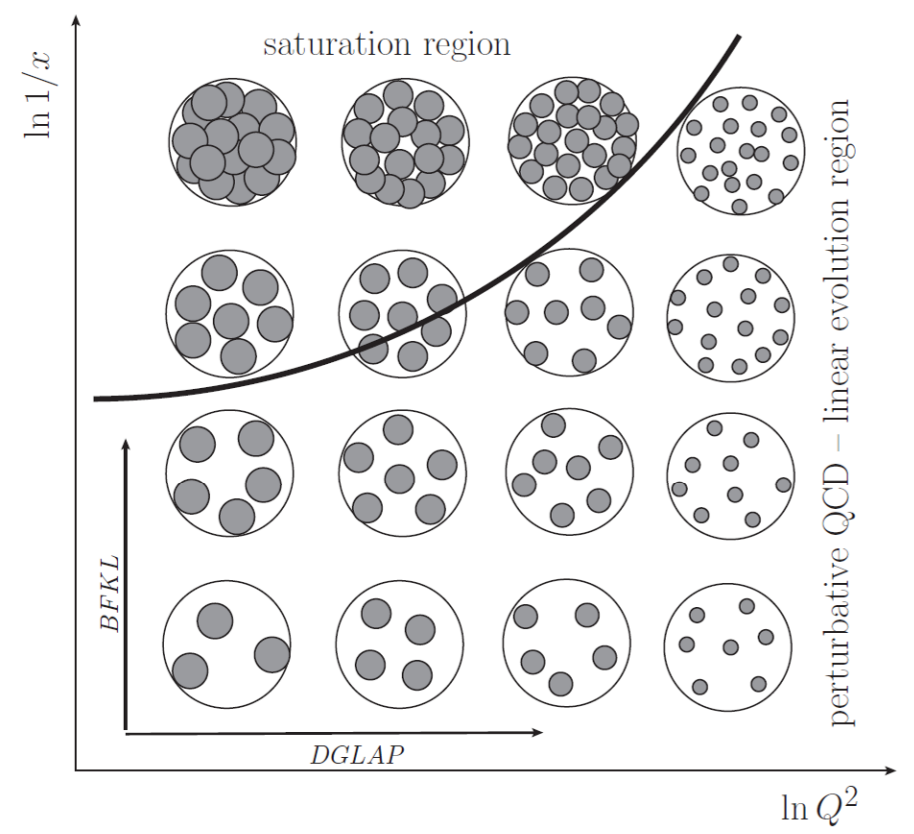

Figura 1.4: Evoução da estrutura partônica do próton no plano $\left(\ln \left(Q^{2}\right), \ln (1 / x)\right)$. A curva preta denota a chamada "escala de saturação". Figura extraída de [106].

A formação de um sistema partônico denso onde os gluons começam a se sobrepor e, por conterem carga de cor não nula, a interagirem entre si conduz, por exemplo, à ocorrência de múltiplos espalhamentos partônicos (isto é, ao invés de um párton do projétil interagir uma única vez com um párton do alvo, existe uma probabilidade, não nula, de que o párton projétil sofra reespalhamentos ao atravessar um meio partônico denso). A alta densidade de gluons também conduz à modificação das equações de 
evolução padrão da QCD, que se tornam não lineares devido ao surgimento de um novo fenômeno, conhecido como "saturação de pártons" (que deveremos tratar nos capítulos subsequentes). Na Figura 1.4, essa região é aquela acima da curva preta no plano $\left(\ln \left(Q^{2}, \ln (1 / x)\right)\right)$.

A física utilizada para a descrição de sistemas densos difere daquela aplicada à descrição de sistemas diluídos e é um ponto crucial nesta tese. Uma descrição da física utilizada para a caracterização de sistemas partônicos densos será apresentada no Capítulo 2. Por hora, no intento de explanarmos nossos estudos quanto às equações de evolução da QCD, é importante mencionar que existe uma outra equação de evolução linear que considera apenas a evolução na variável de Bjorken, $x$. Esta equação é conhecida equação Balitsky-Fadin-Kuraev-Lipatov ou, simplesmente, BFKL e será discutida brevemente a seguir.

\subsubsection{A equação BFKL}

O limite de altas energias dos processos descritos pela QCD é equivalente ao limite de pequenos valores de $x$, como visto na equação (1.16). Nesse limite, de pequenos valores de $x$, as equações DGLAP, introduzidas na seção anterior, estão fora da sua região de aplicabilidade. As soluções das equações DGLAP são válidas na região onde o parâmetro $\alpha_{s}\left(Q^{2}\right) \ln \left(Q^{2} / \Lambda_{Q C D}\right)$ domina a série perturbativa (ou seja, para grandes valores de $Q^{2}$ ). Todavia, para um valor suficientemente pequeno de $x$, os termos proporcionais a $\alpha_{s} \ln (1 / x)$ se tornam maiores que os termos $\alpha_{s} \ln \left(Q^{2} / \Lambda_{Q C D}\right)$ e apenas as divergências em logarítmos de $1 / x$ devem ser ressomadas ${ }^{8}$.

A equação mais simples que trata as divergências em logaritmos de $1 / x$ é a equação BFKL [19-21]. Esta equação fornece a evolução das distribuições não integradas de gluons para um valor qualquer de $x$ assumindo-se que estas distribuições são conhecidas em um dado valor inicial $x_{0}$. A equação BFKL para a distribuição de gluons não

\footnotetext{
${ }^{8}$ Outra maneira de enxergar isso é manter $Q^{2}$ fixo no limite de DLLA nas equações DGLAP, dessa maneira o parâmetro de ressoma passa a ser $\alpha_{s} \ln (1 / x) \sim 1$.
} 
integrada é dada por [19-21, 101]

$$
\frac{\partial \phi\left(x, k_{\perp}^{2}\right)}{\partial \ln (1 / x)}=\frac{\alpha_{s} N_{c}}{\pi^{2}} \int \frac{d^{2} q_{\perp}}{\left(k_{\perp}-q_{\perp}\right)^{2}}\left[\phi\left(x, k_{\perp}^{2}\right)-\frac{k_{\perp}^{2}}{2 q_{\perp}^{2}} \phi\left(x, k_{\perp}^{2}\right)\right]
$$

onde $\hat{g}\left(x, k_{T}^{2}\right)=\phi\left(x, k_{T}^{2}\right) / k_{T}^{2}$, conforme a equação (1.49).

Uma observação importante oriunda da solução da equação BFKL é o rápido crescimento da distribuição de gluons com a diminuição de $x$,

$$
\phi\left(x, k_{\perp}^{2}\right) \sim\left(\frac{1}{x}\right)^{\lambda}
$$

onde

$$
\lambda=\frac{4 \alpha_{s} N_{c}}{\pi} \ln 2
$$

Sua solução fornece um crescimento mais forte para a distribuição de gluons do que aquele obtido no limite de pequenos valores de $x$ na aproximação de DLLA. No entanto, conforme mencionado anteriormente, no limite de pequenos valores de $x$ e $Q^{2}$ fixo, o processo de emissão de gluons se encarrega de aumentar o número de gluons dentro de um dado hádron colidente sem que a área ocupada por cada um deles seja alterada; ou seja, o "tamanho"transversal de cada gluon será $r_{\perp} \sim 1 / k_{T}$. Em algum momento isso levará à inevitável sobreposição dos gluons no plano transversal e, portanto, a interações entre esse gluons. As interações entre gluons no regime de pequenos valores de $x$ não são descritas pela equação BFKL.

No hádrons colidentes o crescimento desenfreado da função de distribuição de gluons implica em um aumento igualmente desenfreado no número de gluons quando valores de $x$ cada vez menores são acessados, o que é possível em colisões a altas energias. Nessas condições, a estrutura interna do próton forma um meio gluônico extremamente denso e colorido [35-43,57,58]. Entretanto, este crescimento não pode continuar indefinidamente pois, uma vez que os objetos colidentes possuem um tamanho finito, o número de pártons a serem resolvidos dentro destes não pode ocupar uma área maior que suas próprias áreas. Em outras palavras, um número infinito de 
pártons ocuparia uma área infinita. Por

Deixando de lado este argumento puramente geométrico, como será visto adiante, uma consequência muito mais importante do crescimento desenfreado da distribuição de gluons a pequenos valores de $x$ é que este fato também conduziria ao crescimento da seção de choque hadrônica numa taxa que viola a unitaridade da matriz de espalhamento (ou seja, a probabilidade máxima de interação deve ser igual 1) e/ou o chamado limite de Froissart [107],

$$
\sigma_{\text {tot }}^{p p(\bar{p})}(s \rightarrow \infty) \leq C \log ^{2}\left(s / s_{0}\right)
$$

que determina a taxa máxima que a seção de choque total $p p$ pode possuir sem que haja violação da unitaridade. Na equação acima $s$ representa o quadrado da energia do centro de massa do espalhamento $p p, s_{0}$ representa uma escala de energia a ser determinada e $C$ representa uma constante.

Existem ainda outros problemas que contribuem para a não validade das equações de evolução usuais da QCD em determinados regimes de $x$ e $Q^{2}$, como o aparecimento de distribuições de gluons negativas [108] e o indício [33,34, 109, 110] da quebra do teorema da fatorização colinear [111], um dos alicerces das equações DGLAP [101].

O próximo capítulo desta tese será dedicado à caracterização da física de sistemas densos, onde a dinâmica linear das equações de evolução da QCD apresentadas neste capítulo sofrem modificações e tornam-se não lineares devido ao surgimento de um novo e importante fenômeno conhecido como "saturação de pártons", que ocorre devido ao alto número de gluons no interior dos hádrons colidentes. O ferramental teórico adequado para o tratamento desse tipo de sistema formado em colisões a altas energias também será apresentado no próximo capítulo. 


\section{Capítulo 2}

\section{A Física de Sistemas com Altas Densidades Partônicas}

Neste capítulo apresenta-se uma discussão do conceito de saturação de pártons bem como as possíveis indicações experimentais que dão suporte a sua existência. Aqui também se encontram as descrições das abordagens e dos ingredientes utilizados para a implementação e o estudo dos efeitos da saturação nos observáveis físicos por nós considerados e que serão apresentados nos próximos capítulos.

\subsection{A saturação de pártons}

As medidas que levaram à comprovação da violação do scaling na variável de Bjorken realizadas no HERA [22-32] mostraram também, através da observação do crescimento da função de estrutura, $F_{2}\left(x, Q^{2}\right)$, e de sua derivada, $\partial F_{2} / \partial \ln Q^{2}$, com o aumento de $Q^{2}$, que conforme $x$ decresce, as distribuições de quarks do mar e, principalmente, a distribuição de gluons crescem muito fortemente. A Figura 2.1 mostra a análise da distribuição de gluons em função de $x$ para valores fixos de $Q^{2}$ efetuada pela colaboração H1 [27]. 


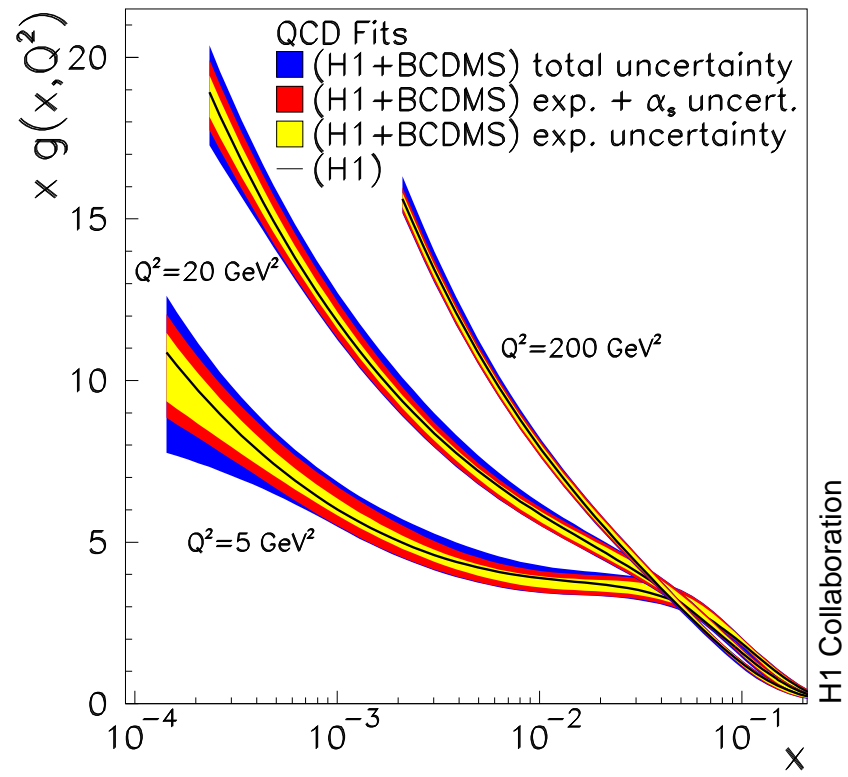

Figura 2.1: Distribuição de gluons obtidas a partir dos dados experimentais do HERA [27].

O crescimento do número de gluons pode ser entendido como um efeito cascata, onde os próprios gluons podem emitir outros gluons de menor momento; além disso, os gluons também podem ser emitidos por quarks. Como discutido no capítulo 1, o forte crescimento da densidade de gluons faz com que o próton seja visto como um meio altamente denso e colorido a altas energias.

As equações de evolução lineares, como a DGLAP e a BFKL, assumem implicitamente que a altas energias o processo mais importante a ser considerado é o de emissão de gluons, prevendo assim, como já apontado, um crescimento exacerbado da distribuição de pártons no interior dos prótons. Nessas equações gluons são emitidos de maneira independente, de modo que a interação entre eles não é considerada. No entanto, em 1983, Gribov, Levin e Ryskin [33] apresentaram a ideia de que a altas energias o processo de recombinação de gluons também passaria a ser importante devido à alta densidade partônica nos hádrons colidentes. Em outras palavras, a interação entre os gluons no interior dos hádrons colidentes também deveria ser levada em consideração. 
O processo de recombinação de gluons pode ser entendido como o processo contrário ao processo de emissão: no processo de recombinação dois gluons de baixo momento se fundem, $g g \rightarrow g$, diminuindo assim a população de gluons no próton. Enquanto o processo de emissão tem a função de aumentar a população de gluons, a inclusão do processo de recombinação coíbe o crescimento desenfreado das distribuições de gluons através de um mecanismo que ficou originalmente conhecido como "saturação de pártons".

No regime de saturação a quantidade de gluons dentro dos hádrons seria tão grande que o processo de recombinação de gluons, não incluídos nas equações de evolução lineares, seria tão ativo quanto os processos de emissão, fazendo com que a distribuição de gluons parasse de crescer indefinidamente e passasse a flutuar em torno de um dado valor, isto é, saturasse em um valor máximo. Conforme será visto mais adiante, a inclusão dos processos de recombinação altera as equações de evolução padrão da QCD, tornando-as não lineares.

Pode-se estimar a escala em que os efeitos da saturação de pártons passam a ser importantes de uma maneira simples. Em colisões de íons relativísticos, quando um núcleo (de $A$ nucleons e grande momento longitudinal) sofre contração de Lorentz, os pártons (predominantemente gluons) contidos nos núcleos se distribuem sobre uma fina casca no plano transversal. Uma vez que os gluons são provados em uma escala $Q^{2}$, pelo princípio da incerteza, cada párton ocupa uma área transversal $\sim 1 / Q^{2}$. Ao mesmo tempo, a seção de choque depende do número de gluons, $A x g\left(x, Q^{2}\right)$, de modo que a área transversal total ocupada pelos gluons que são provados numa escala $Q^{2}$ é dada por $A x g\left(x, Q^{2}\right) / Q^{2}$. Nessas condições, os gluons nos núcleos colidentes começam a se sobrepor e a interagir quando a área transversal ocupada por eles é da ordem da área transversal do núcleo, $S_{\perp}$. Para um alvo nuclear essa área transversal é proporcional a $A^{2 / 3}$, de modo que $S_{\perp}=A^{2 / 3} S_{0 \perp}$, onde $S_{0 \perp}$ é a área transversal de um próton. Finalmente, como a probabilidade de que os gluons interajam é proporcional a constante de acoplamento forte, $\alpha_{s}$, tem-se que a interação entre os gluons se torna 
importante na escala

$$
Q_{s}^{2}(x) \sim \alpha_{s} \frac{A}{A^{2 / 3}} \frac{x g\left(x, Q^{2}\right)}{Q^{2} S_{0 \perp}} \sim A^{1 / 3}\left(\frac{1}{x}\right)^{\lambda}
$$

onde utilizou-se a equação (1.57) para o comportamento da distribuição de gluons em pequenos valores de $x$.

O fenômeno da saturação introduz assim uma nova escala de momento, $Q_{s}$, conhecida como "escala de saturação" e uma vez que a área ocupada por cada gluon é proporcional a $1 / Q^{2}$, os efeitos da recombinação de gluons (que conduzem ao fenômeno da saturação) se tornam importantes na região $Q^{2} \leq Q_{s}^{2}$. É importante ressaltar que a violação da unitaridade da matriz de espalhamento ocorre nessa mesma região caso os efeitos de saturação não sejam considerados. Além disso, é possível observar que para pequenos valores de $x$ tem-se que $Q_{s} \gg \Lambda_{Q C D}$ e a teoria perturbativa é aplicável, com a escala de saturação servindo como escala perturbativa.

Acredita-se hoje que, no domínio de altas energias e/ou altas densidades, a dinâmica da QCD torna-se qualitativamente diferente da dinâmica no regime de curtas distâncias e de altos momentos transferidos. Neste primeiro domínio, que é de extremo interesse para esta tese, espera-se a formação de um novo estado da matéria denominado de "Condensado de Vidro de Cor" (Color Glass Condensate - CGC) [33-58]. A formação desse novo estado da matéria vem sendo descrita em termos da saturação de pártons e, conforme já mencionado, as equações de evolução da QCD se tornam não lineares na medida em que efeitos de coerência, como múltiplos espalhamentos, devem ser considerados devido à alta densidade partônica.

Atualmente existem diferentes abordagens que tentam incluir os efeitos do fenômeno da saturação de gluons nos cálculos. Estas abordagens possuem diferentes graus de complexidade e vão desde uma simples alteração no espaço de fase até a construção de uma teoria efetiva [33-58].

Antes de seguirmos faz-se necessária uma explicação para o termo CGC. A palavra "condensado" advém vem da alta densidade de gluons presente na função de onda 
hadrônica que, por conta disso, pode ser tratada através de um formalismo que leva em consideração fortes campos (de cor) clássicos" ${ }^{1}$ O termo "vidro"surge de detalhes técnicos da formulação da teoria efetiva do CGC, onde uma separação dos graus de liberdade da teoria é assumida: gluons com grande fração de momento, $x$, atuam como fontes emissoras, dando origem a gluons com pequenas fração de momento, $x^{\prime}(\ll x)$. De fato, é possível mostrar que o tempo de vida de cada gluon emitido é proporcional à sua fração de momento e, desta maneira, gluons que carregam uma pequena fração de momento vêm gluons com grande fração de momento como fontes emissoras estacionárias. Esse procedimento é análogo à separação dos graus de liberdade de acordo com as escalas de tempo assumida na teoria de vidros de spin [112]. Por fim, o termo "cor" advém do fato dos gluons da QCD serem portadores de carga de cor não nula.

A seguir vamos apontar algumas medidas experimentais onde acredita-se existir algum indício dos efeitos da saturação de pártons. Posteriormente detalham-se as abordagens e ingredientes teóricos que foram utilizados nesta tese para a inclusão deste efeito na descrição teórica dos observáveis por nós estudados.

\subsection{Indícios experimentais da saturação de pártons}

Em 1999 os autores da referência [113] afirmaram que alguns efeitos da saturação já estariam sendo observados no HERA. A partir de 2000, com o início das operações do RHIC, a procura por sinais da formação do CGC se tornou mais intensa e surgiram inúmeros trabalhos propondo novos observáveis onde os efeitos da saturação pudessem ser inferidos sem ambiguidade.

Em 2004 os resultados do RHIC obtidos em colisões dêuteron-ouro $(d A u)[114,115]$ levaram muitas pessoas a acreditar que o CGC havia sido inequivocamente observado.

\footnotetext{
${ }^{1}$ Embora a abundante quantidade de gluons com pequena fração de momento, $x$, não formem um condensado no sentido de um condensado de Bose-Einstein (onde a maior parte dos bósons ocupam o estado fundamental) a analogia torna-se pertinente devido o grande número de ocupação em ambos os casos.
} 
Porém, questionamentos acerca dessa importante conclusão foram progressivamente levantados quanto aos possíveis sinais experimentais da formação do CGC. Entre eles, como assinalado na ref. [59], destacam-se:

1. o scaling geométrico da seção de choque fóton-próton $\left(\gamma^{*} p\right)$ medida no HERA, caracterizado pela dependência na seção de choque na variável $\tau=Q^{2} / Q_{s}^{2}(x)$;

2. o comportamento da função de estrutura $F_{2}$ medida em DIS no HERA;

3. o comportamento da razão entre as seções de choque difrativa e total medidas no HERA;

4. o comportamento da seção de choque de fotoprodução do méson $\rho$ medida no HERA;

5. o comportamento da multiplicidade de partículas carregadas medidas na região central do RHIC;

6. o comportamento da distribuição de momento transversal das partículas produzidas em colisões $d-A u$ no RHIC na região de grandes momentos e grande rapidez.

Posteriormente surgiram trabalhos [60-62] mostrando que os observáveis mencionados acima também poderiam ser descritos sem a hipótese da saturação de pártons e a formação do CGC. Nas próximas seções os itens 1 e 5 listados acima (bem como resultados mais recentes, obtidos no LHC e que dão suporte ao fenômeno da saturação) serão discutidos em maiores detalhes pois, quando comparados aos demais itens, são mais relevantes para esta tese.

De fato, se levarmos em consideração apenas os dados experimentais coletados no HERA não é possível confirmar a formação do CGC, uma vez que o espaço de fase acessado por seus detectores não é extenso o suficiente para distinguir univocamente as descrições que supõem ou não a formação do CGC. À época, a expectativa passou 
a ser que com um melhor conhecimento das distribuições de gluons na região de pequenos valores de $x$ fornecida pelos dados experimentais vindouros do HERA e com a construção de um novo acelerador de partículas, o LHC, a formação do CGC poderia vir a ser confirmada experimentalmente.

O LHC está em operação desde outubro de 2008 e embora já tenha fornecido diversas medidas experimentais importantes em regiões cinemáticas até então inexploradas, até o presente momento nenhuma delas fornece uma "assinatura" clara e definitiva da formação do CGC.

Embora o LHC esteja atualmente operando na faixa de energia de $13 \mathrm{TeV}$ em colisões $p p$ [116], valor muito próximo de sua energia máxima, $14 \mathrm{TeV}$, e exista um consenso de que para que se comprove a formação do CGC, sem que haja ambiguidades entre as descrições com e sem a sua formação, faz-se necessária a construção de um colisor elétron-íon.

Um dos motivos pelo qual essa assinatura provavelmente ainda não tenha sido encontrada no LHC vem do fato de que a física do CGC se desenvolve nos estados iniciais do processo de colisão que propiciam a formação de um meio muito "sujo", que dificulta a procura por sinais remanescentes desta etapa do processo. Por exemplo, em colisões $A A$ espera-se a formação do Plasma de Quarks e Gluons (Quark Gluon Plasma - QGP), um meio altamente interagente que dificulta a detecção dos sinais da formação do CGC, uma vez que introduz uma série de efeitos de estado final (veja [117] para um artigo de revisão recente).

Outro motivo está no fato de que os limites cinemáticos, possíveis de serem alcançados no HERA em DIS, não foram suficientes para diminuir as incertezas nas distribuições de gluons quando extrapoladas a baixíssimos valores de $x$, gerando espaço para acomodar diferentes descrições de um mesmo observável utilizando-se, porém, diferentes suposições. Resguardadas as frustrações, o espaço de fase alcançado pelos detectores do HERA revelou importantes indicações sobre o comportamento qualitativo de alguns observáveis numa região cinemática próxima ao regime de saturação e inúmeros indícios da presença dos efeitos característicos dessa física, têm sido analisa- 
dos e debatidos até o presente momento.

Atualmente discute-se a construção de um novo colisor elétron-íon. Três propostas foram recentemente apresentadas, duas nos Estados Unidos e uma na Europa [118-120]. Processos de DIS fornecem uma prova mais "limpa" ${ }^{2}$ dos efeitos da QCD a altas energias e, a comunidade científica dedicada ao estudo dos efeitos da saturação, acredita que grandes avanços podem ser feitos em um colisor elétroníon. Nas referências [118-120] são apresentadas as principais medidas experimentais que os novos detectores seriam capazes de fornecer e que contribuiriam para o avanço significativo do nosso conhecimento sobre diversos fenômenos físicos que ocorrem a altíssimas energias, dentre eles o da saturação.

A seguir, os resultados 1 e 5 da lista apresentada anteriormente, bem como outros resultados mais recentes obtidos em colisões hadrônicas no LHC serão discutidos em maiores detalhes.

\subsubsection{O scaling geométrico}

O scaling geométrico, introduzido pela primeira vez no contexto do chamado " $m o$ delo de dipolo de cor" (vide seção 2.3 e suas subseções) proposto por Golec-Biernat e Wüsthoff (GBW) [113], conduz a uma notável propriedade verificada nos dados experimentais da seção de choque $\sigma_{\gamma^{*} p}$ medida em eventos de DIS no HERA. Essa propriedade será descrita de maneira sucinta a seguir. Para maiores detalhes (como expressões matemáticas para as quantidades físicas mencionadas a seguir) indica-se a o trabalho original onde os principais resultados foram apresentados [121].

Em [121] foi apontado que, uma vez desprezada a massa dos quarks na função de onda dos fótons, a seção de choque $\sigma_{\gamma^{*} p}\left(x, Q^{2}\right)$ passa a depender apenas da combinação $\tau=Q^{2} R_{0}^{2}(x)$, com $R_{0}^{2}(x)=\left(x / x_{0}\right)^{\lambda} / Q_{0}^{2}\left(x_{0} \sim 3 \times 10^{-4}, Q_{0}=1 \mathrm{GeV}\right.$ e $\left.\lambda=0,2 \div 0,3\right)$, na região de pequenos valores de $x(<0,01)$ e $0,045 \mathrm{GeV}^{2}<Q^{2}<450 \mathrm{GeV}^{2}$ e não mais

\footnotetext{
${ }^{2}$ O DIS não propicia a formação do QGP e, portanto, efeitos de estado final não se encontram presentes.
} 
das variáveis $Q^{2}$ e $x$ separadamente. É importante notar que a variável de scaling, $\tau$, também pode ser escrita como $\tau=Q^{2} / Q_{s}^{2}(x)$, lembrando que pelo princípio de incerteza tem-se que $R_{0} \sim 1 / Q_{s}$.

A Figura 2.2 mostra os dados experimentais da seção de choque $\sigma_{\gamma^{*} p}$ em dois regimes distintos, para pequenos valores de $x(<0,01)$ e para grandes valores de $x$ (> 0.01) como função da variável de scaling $\tau$. Como pode ser observado, na região de pequenos $x$ os dados experimentais se sobrepõem em uma única curva, ou seja, apresentam um "scaling". Entretanto, na região de grandes valores de $x$, a propriedade de scaling deixa de ser válida. Esta propriedade também foi encontrada em eventos de DIS difrativos [122-125] e DIS nuclear (a energias menores que aquelas acessadas no HERA) [126]. Este comportamento de scaling é uma manifestação da ideia de que a única escala dimensional relevante a altas energias (e pequenos valores de $x$ ) é a escala de saturação.
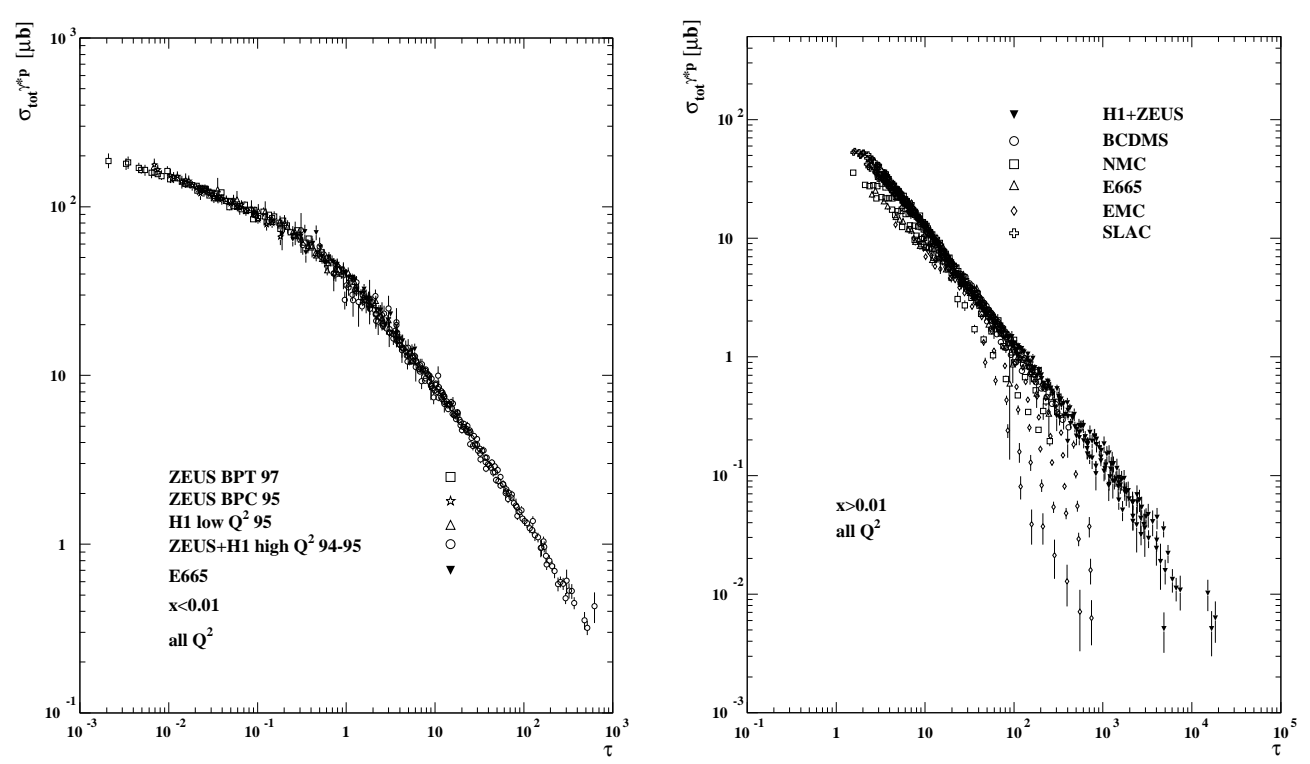

Figura 2.2: Seção de choque do DIS para $x<0.01$ (esquerda) e $x>0.01$ (direita) em função da variável $\tau=Q^{2} R_{0}^{2}(x)$ onde $0,045 \mathbf{G e V}^{2}<Q^{2}<450 \mathbf{G e V}^{2}$. Os dados experimentais para pequenos valores de $x$ apresentam a propriedade do scaling geométrico enquanto que os dados experimentais para grandes valores de $x$ não apresentam esta propriedade. Figuras extraídas de [121]. 
Existe ainda uma região de grandes valores de $Q^{2}$ para $x$ fixo onde a densidade de gluons é pequena e a pQCD é aplicável. Na referência [127] os autores encontraram uma terceira região, intermediária às regiões de alta e baixa densidade, onde existem soluções universais das equações do grupo de renormalização que satisfazem a propriedade de scaling. Esta região, cujo o alcance é $Q_{s}^{2} \leq Q^{2} \leq Q_{s}^{4} / \Lambda_{Q C D}^{2}$, ficou conhecida como "região de scaling estendida".

O scaling geométrico é uma consequência direta do comportamento das soluções (assintóticas) das equações de evolução para a distribuição de pártons no próton no regime de saturação e também é uma propriedade suportada pelos dados experimentais de HERA, por exemplo. Por conta disso, essa propriedade também é incluída na maior parte dos modelos de dipolo propostos na literatura.

A propriedade de scaling geométrico verificada nos dados experimentais de HERA foi motivada pelo modelo de dipolo da referência [113]. Em geral os modelos de dipolo são válidos na região de pequenos valores de $x$ e até valores moderados de $Q^{2}$, já que não incluem a evolução em $Q^{2}$, ditada pelas equações DGLAP, algo essencial para a descrição dos dados de HERA. Todavia, uma vez que a propriedade de scaling geométrico nos dados experimentais de HERA é válida em uma região muito grande de $Q^{2}$, muito além do regime de validade destes modelos, foi argumentado que esta seria uma propriedade mais geral que o modelo de dipolo que inspirou a procura pelo mesmo scaling nos dados experimentais.

Posteriormente, foi demonstrado que é possível obter a propriedade de scaling geométrico a partir das equações DGLAP [63] e, portanto, sem assumir a existência da saturação de pártons. Neste caso, no entanto, a propriedade de scaling é válida apenas na região de $Q^{2} \gtrsim 10 \mathrm{GeV}^{2}$ e, portanto, a presença do scaling encontrado nos dados experimentais na região de $Q^{2} \lesssim 5 \mathrm{GeV}^{2}$ talvez possa ser uma evidência genuína dos efeitos da saturação de partons, já que não é possível ser obtido a partir de uma equação de evolução linear.

Recentemente, a existência da propriedade de scaling geométrico também foi investigada nos dados experimentais obtidos em colisões $p p$ no LHC e $A A$ no RHIC. 
Em [128] os autores mostraram que a dependência com a energia da multiplicidade de partículas carregadas na região central de pseudorapidez $(\eta), d N(\sqrt{s}) /\left.d \eta\right|_{\eta=0}$ e do momento transversal médio de partículas carregadas, $\left\langle p_{T}(\sqrt{s})\right\rangle$, podem ser descritos pela teoria do CGC. Em um estudo posterior [129], os autores demonstraram a existência de um scaling na distribuição de momento transversal das partículas carregadas, $d N_{c h} / d \eta / d^{2} p_{T}$. Se a escala de saturação fosse a única escala a controlar a distribuição de momento transversal, essa quantidade deveria exibir o seguinte scaling [129],

$$
\frac{d N_{c h}}{d \eta d^{2} p_{T}}=\frac{1}{Q_{0}^{2}} F(\tau)
$$

que é baseado em argumentos de análise dimensional, onde $Q_{0}^{2}(\sim 1 \mathrm{GeV})$ é uma escala de momento fatorizada, por conveniência.

Como mostra a Figura 2.3, a propriedade de scaling geométrico também se manifesta na distribuição de momento de momento transversal de partítulas carregadas produzidas em colisões $p p$ nas energias do LHC e em colisões $A A$ nas energias do RHIC. Neste caso a variável de scaling é $\tau=\left(p_{T} / Q_{s}\right)^{2}$, onde $Q_{s}^{2}\left(p_{T}, \sqrt{s}\right)=$ $A^{1 / 3} Q_{0}^{2}\left[p_{T} /\left(\sqrt{s} \times 10^{-3}\right)\right]^{-\lambda}$, com $Q_{0}=1 \mathrm{GeV}$ e o valor de $\lambda$ encontra-se especificado nas figuras. É interessante notar que a qualidade do scaling é menor em colisões $A A$ devido à presença de efeitos de estado final, como os que ocorrem quando da formação do QGP.

\subsubsection{Observáveis integrados no momento transversal}

Uma quantidade abundante de partítulas são criadas em colisões hadrônicas que cresce com o número atômico dos núcleos envolvidos, como pode ser observado na Figura 2.3 (b). Considerando-se a Figura 2.3 (a), vê-se que a maior parte das partículas produzidas encontra-se na região $\tau \lesssim 1.25$. Utilizando-se a variável de scaling da seção anterior, $\tau=\left(Q \equiv p_{T} / Q_{s}\right)^{2}$, tem-se que a região de momento transversal correspondente a este valor de $\tau$ é $p_{T} \lesssim(0.99 \times 1.25)^{2 /(2+\lambda)} \lesssim 1.2 \mathrm{GeV}$, para $\sqrt{s}=900$ 


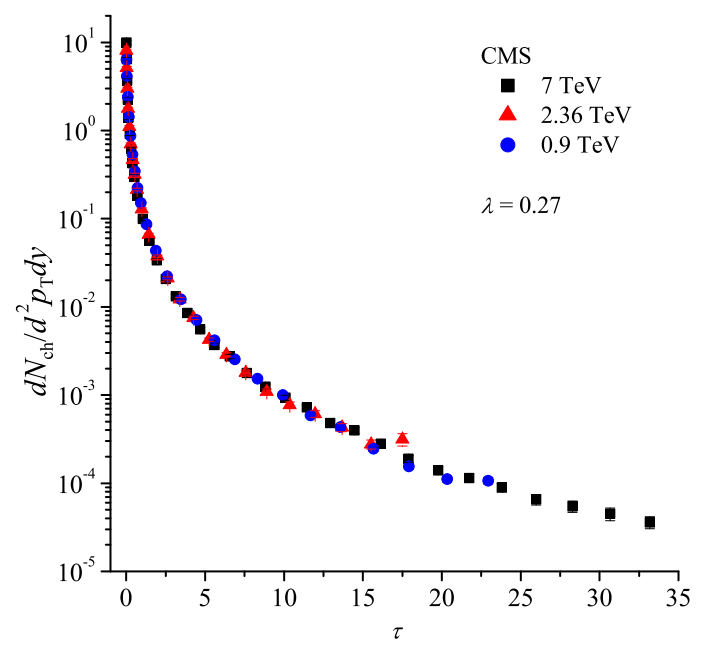

(a)

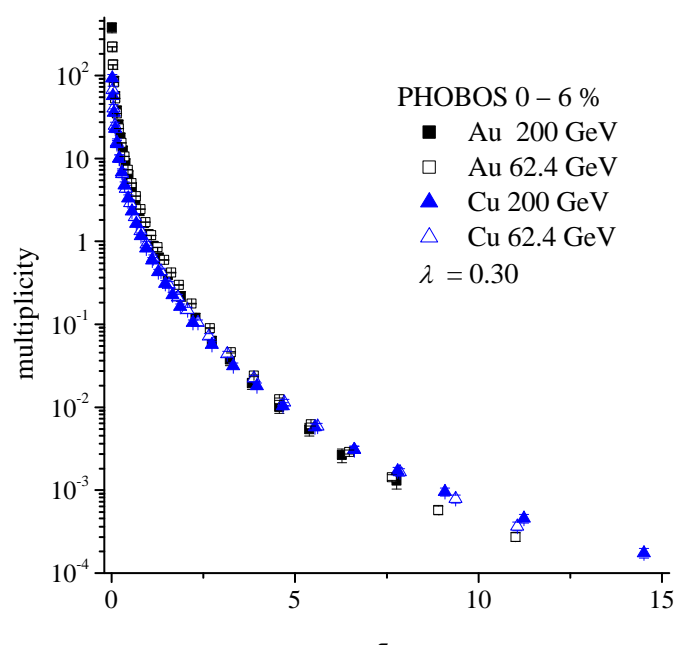

(b)

Figura 2.3: Propriedade de scaling geométrico observada nos dados experimentais para a distribuição de momento transversal de partítulas carregadas produzidas em (a) colisões $p p$ nas energias do LHC e (b) colisões $A A$ nas energias do RHIC plotadas como função da variável $\tau=\left(p_{T} / Q_{s}\right)^{2}$. Figuras retiradas de $[129,130]$.

$\mathrm{GeV}$.

Sendo assim, quando observáveis são resultados de integrações no momento transversal das partículas produzidas, as principais contribuições são provenientes dessa mesma região ou oriundas daquelas de menores valores de momento transversal. Em uma aproximação não muito grosseira, essa região é exatamente a região não perturbativa, onde a teoria padrão da QCD deixa de ser válida, pois prediz um crescimento muito forte do conteúdo partônico nos hádrons.

De fato, abordagens que se apoiam apenas nas equações de evolução padrão da QCD, as equações DGLAP e BFKL apresentadas no capítulo anterior, não são capazes de descrever tais observáveis em todo o regime cinemático ${ }^{3}$. Para que isso seja possível é necessário utilizar uma abordagem que incorpore os efeitos da física não perturbativa

\footnotetext{
${ }^{3}$ É oportuno lembrar que a violação da unitaridade da matriz de espalhamento ocorre na região $Q \leq Q_{s}$ e, portanto, abordagens apoiadas apenas em tais equações violariam essa propriedade na tentativa de descrição de observáveis integrados no momento transversal.
} 
para um dado valor de momento transversal, onde essas equações deixam de ser válidas devido à presença de efeitos não lineares, como os efeitos de saturação e de coerência, como a ocorrência de múltiplos espalhamentos (maiores detalhes sobre esse ponto serão dados na seção 2.4).

Por outro lado, na teoria do CGC tem-se que, por exemplo, a multiplicidade de partículas carregadas produzidas em colisões $p p$ e $A A$ por unidade de área transversal, $S_{\perp}$, deve ser proporcional à escala de saturação ao quadrado [33,131-133],

$$
\frac{1}{S_{\perp}} \frac{d N}{d y} \sim Q_{s}^{2}
$$

uma vez que esta é a única escala que carrega dimensão na teoria.

Este fato leva à predição de que a multiplicidade de partículas deveria crescer com $Q_{s}^{2}$ como uma potência da energia envolvida no processo. A Figura 2.4 (a) mostra uma compilação dos dados experimentais para o comportamento da multiplicidade de partículas carregadas produzidas na região central de pseudorapidez com a energia assim como os resultados da referência [134], obtidos com uso da teoria do CGC. Outro importante exemplo do poder da teoria do CGC com respeito ao cálculo de observáveis integrados no momento transversal é apresentado na Figura 2.4 (b). Nesta Figura encontra-se a comparação dos resultados obtidos via teoria do CGC com a distribuição de rapidez, $y$, para uma energia fixa e diversas classes de centralidade (colisões centrais envolvem um grande número de participantes e esse número decresce com o aumento da centralidade, significando colisões mais periféricas). Os observáveis aqui mencionados serão estudados em maiores detalhes na seção 4.5.

Como último exemplo a ser mencionado nessa seção, a multiplicidade de partículas carregadas e normalizada pelo par de nucleons participantes como função da centralidade (representada pelo número de participantes para uma dada centralidade) para uma energia fixa é apresentada na Figura 2.5. As três curvas, de baixo para cima, apresentadas na figura são predições baseadas na física do CGC, cada uma com as suas particularidades. Em especial, a predição apontada na referência [135], identifi- 


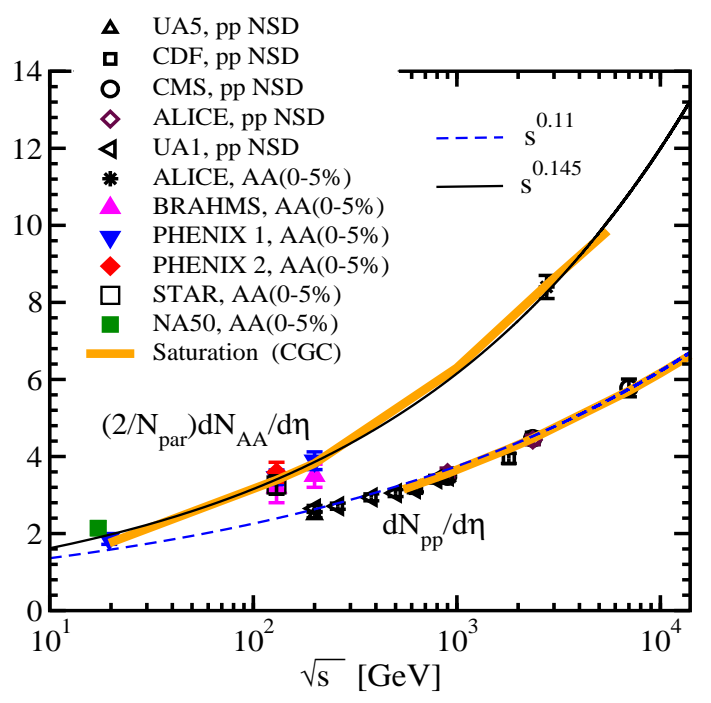

(a)

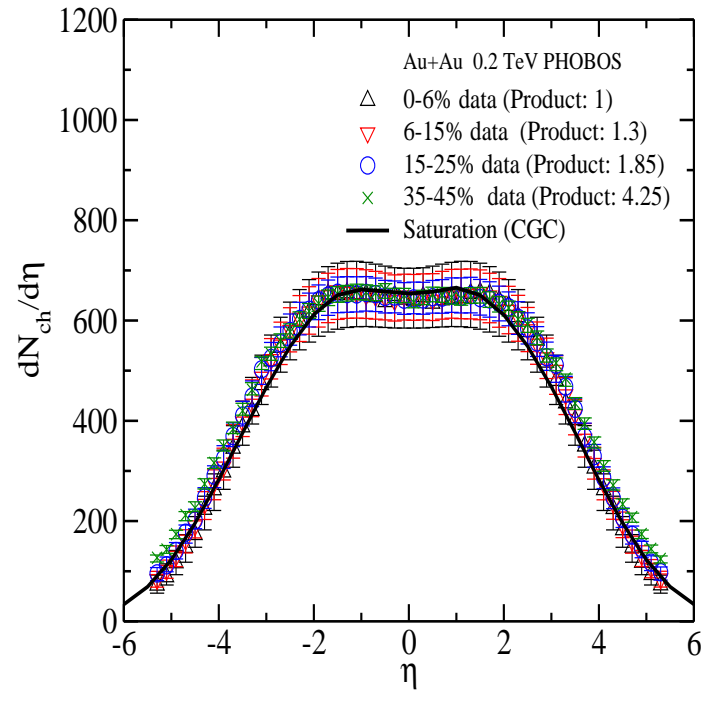

(b)

Figura 2.4: (a) Comportamento da multiplicidade de partículas carregadas produzidas em $\eta=0$ normalizada pelo número de pares de partículas participantes em colisões $A A$ (curva e pontos experimentais superiores) e em colisões $p p$ (curva e pontos experimentais inferiores) com a energia (b) Distribuição de rapidez em colisões $A u A u$ em $\sqrt{s}=200 \mathrm{GeV}$ para diversas janelas de centralidade. Figuras retiradas de [134].

cada como Albacete et. al., descreve os dados experimentais com uma boa qualidade. As três primeiras curvas desta figura, de cima para baixo, por outro lado representam predições vindas de simulações de Monte Carlo que não se baseiam na física do CGC.

\subsubsection{O efeito "ridge" e assimetrias angulares em colisões $p p$ e $p A$}

Outro observável que dá suporte à formação do CGC vem do estudo de correlações angulares de longo alcance (em rapidez) no plano de pseudorapidez relativa, $\Delta \eta$, e do ângulo azimutal relativo, $\Delta \phi$, entre duas ou mais partículas (geralmente com pequeno momento transversal, $1<p_{T}<3 \mathrm{GeV}$ ) produzidas em colisões $p p$ e $p A$ de alta multiplicidade. Devido a forma particular dessas correlações, colimadas em torno de 


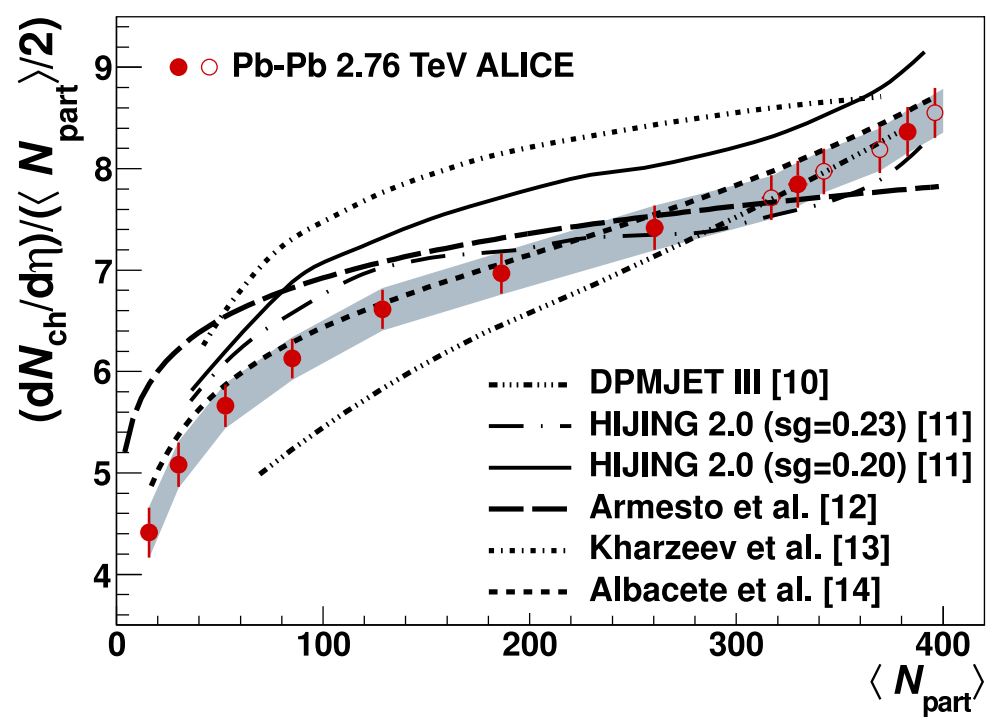

Figura 2.5: (a) Comportamento da multiplicidade de partículas carregadas produzidas em $\eta=0$ normalizada pelo número de pares de partículas participantes em colisões $\mathrm{PbPb}$ em função do número de participantes. Figura retirada de [136].

$\Delta \phi=0$ e se estendendo em um grande intervalo de $\Delta \eta$, esse tipo de estrutura ficou conhecida como efeito "ridge" (ou "cume", em português) na literatura.

Mais precisamente, a observação de correlações que se estendem longamente em pseudorapidez para $\Delta \phi=0$ se deu primeiramente em colisões $A u A u$ no RHIC [137].

Mais tarde, esse fenômeno tambêm foi observado em colisões PbPb no LHC [138, 139]. Independente do sistema colidente, $p p, p A$ ou $A A$, por argumentos de causalidade [140], discute-se ainda hoje se as correlações de longo alcance na (pseudo)rapidez podem ter sua origem poucos instantes após a colisão ocorrer ou até mesmo na função de onda dos hádrons colidentes [140]. Certamente este fato indicaria uma forte influência da dinâmica que ocorre no estado inicial da colisão.

Acredita-se, no entanto, que em colisões de íons pesados a origem desse fenômeno é bem estabelecida e não vem das correlações de estado inicial mas, sim, da evolução hidrodinâmica do sistema após a colisão em resposta às assimetrias presentes no estado inicial da colisão [141-148]. Em colisões $A A$ não centrais, onde a sobreposição das partículas interagentes tem uma forma elíptica [149,150], os gradientes de pressão são 
maiores ao longo do eixo menor dessa elípse, ocasionando uma expansão de matéria maior nessa direção. As assimetrias presentes no estado inicial da colisão (que são correlações no espaço das posições) são então transformadas em correlações no espaço dos momentos através de efeitos de estado final devido à evolução hidrodinâmica do sistema inicial, ou simplesmente fluxo hidrodinâmico. Como a hidrodinâmica descreve a expansão do sistema como um todo e as correlações que geram o efeito ridge envolvem partículas com grande separação em pseudorapidez, diz-se que esse efeito é um efeito coletivo.

Em colisões de íons pesados, as multiplicidades são extremas e se espera a formação do QGP, um meio termalizado que é descrito pela solução das equações hidrodinâmicas, ocasionando, nessas condições, a perda das possíveis correlações (já no espaço dos momentos) existentes no estado inicial e as correlações observadas nos detectores vem de interações de estado final e da resposta da evolução hidrodinâmica do sistema com relação à geometria inicial da colisão. Particularmente por essa razão, a existência do efeito ridge em colisões $A A$ tem sido apontada como uma evidência da formação do QGP, muito embora o debate sobre essa questão continue em vigência.

A ausência desse tipo de estrutura em colisões $p p$ "minimum bias", isto é, quando os sinais gravados pelos detectores de partículas a cada colisão são analisados levandose em consideração todo o alcance cinemático do detector (sem a aplicação de cortes cinemáticos extras além daqueles intrínsecos ao detector), conduziu a comunidade científica a acreditar que colisões $p p$ serviriam apenas como experimentos de controle para posterior efeito de comparação com os dados de colisões de íons pesados. Porém, esse mesmo tipo de estrutura foi observada em colisões $p p$ de altas multiplicidades [151, 152], e foi então natural procurar pelo mesmo fenômeno em colisões $p A$, onde esse mesmo efeito também foi observado [153,154]. A Figura 2.6 mostra a observação experimental do efeito ridge em eventos de altas multiplicidades em colisões $p p, p P b$ e $\mathrm{PbPb}$.

De modo geral, as equações da hidrodinâmica são derivadas assumindo-se uma larga separação entre as escalas de comprimento macroscópica e microscópica. Isso 

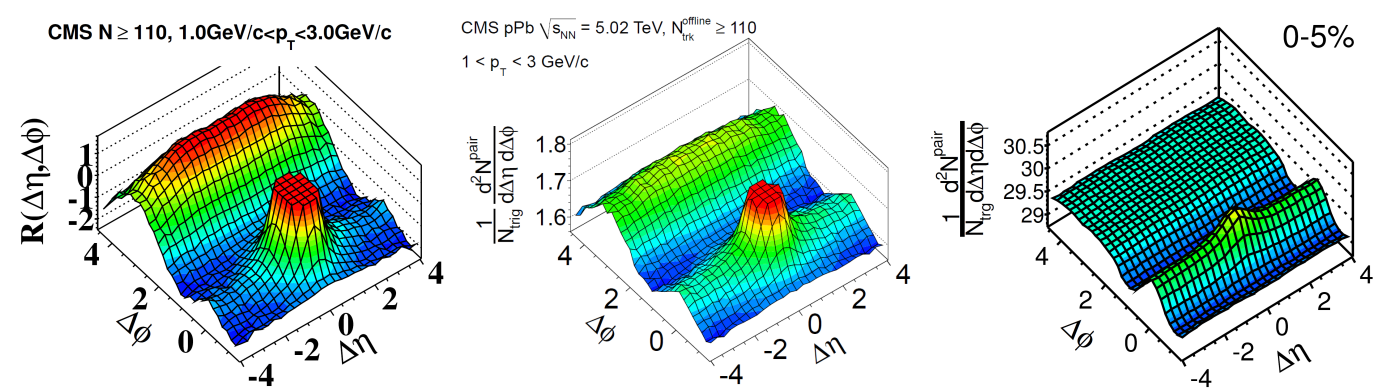

Figura 2.6: Efeito ridge em colisões $p p$ (esquerda), $p P b$ (centro) e $P b P b$ (direita) medidos pela colaboração CMS. $O$ eixo vertical denota a função de correlação entre o número de pares de partículas, $N_{\text {pair }}$ no plano $(\Delta \phi, \Delta \eta)$ normalizada pelo número de partículas que satisfazem o os critérios de seleção aplicados na análise

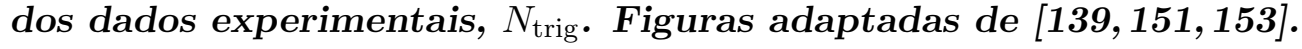

implica que se o tamanho do sistema colidente for diminuído, indo de $A A$ para $p p$, por exemplo, a descrição hidrodinâmica deixaria de ser válida. No entanto, um fenômeno tido como um efeito coletivo foi também observado em colisões de íons pesados, assim como em sistemas "pequenos" $(p p$ e $p A)$. Esse fato gerou o questionamento de se as correlações observadas em um sistema muito menor tinham a mesma origem das correlações observadas em colisões $A A$ ou se a origem física do efeito ridge nesses sistemas era outra.

Em colisões $p A$ (e principalmente $p p$ ) de alta multiplicidade espera-se que, em geral, a densidade de energia depositada no momento da colisão não seja suficiente para formar o QGP e tem-se a esperança de que as correlações de estado inicial não sejam destruídas, podendo ser observadas experimentalmente. Tais eventos, no entanto, são raros e contabilizam apenas entre $1 \%$ e aproximadamente $5 \%$ de todos os eventos analisados pelas colaborações experimentais [151-154]. Sendo assim, parece natural assumir que, nessas condições, provam-se configurações igualmente raras do estado inicial das partículas colidentes.

Assumindo-se que a quantidade de partículas produzidas numa colisão é diretamente correlacionada à densidade do meio partônico nos hádrons colidentes, tem-se, intuitivamente, que os eventos de alta multiplicidade poderiam estar relacionados à co- 
lisão de hádrons no regime denso, onde espera-se a formação do CGC. Nessas condições, as correlações observadas experimentalmente poderiam servir como uma indicação da formação de tal sistema.

Embora fosse esperado que a descrição hidrodinâmica não haveria de ser capaz de descrever bem os dados experimentais relacionados ao efeito ridge nesses casos, devido à dininuição da diferença entre as escalas microscópicas e macroscópicas do sistema colidente e a ausência de efeitos no estado final, esta abordagem é capaz de descrever uma série de medidas experimentais, como, por exemplo, a distribuição de momento transversal e de rapidez de partículas carregadas em colisões $p p$ e $p A$ [155-157] e a dependência das chamadas "assimetrias azimutais" (que serão discutidas no quinto capítulo) com o momento transversal medidas em colisões $p A$ [157-159].

Embora a teoria do CGC também seja capaz de descrever qualitativamente bem o mesmo conjunto de dados experimentais [140,160-177], o mecanismo físico que dá origem as tais correlações é completamente diferente e está relacionado aos fortes campos de cor gerados no estado inicial da colisão e efeitos de coerência nos hádrons colidentes $[140,160,167]$. Isso gera um grande impasse na origem (e portanto no mecanismo físico) por trás do efeito ridge em sistemas pequenos: no CGC essas assimetrias têm origem no estado inicial da colisão, enquanto que na abordagem hidrodinâmica estas mesmas correlações são fruto da expansão anisotrópica do sistema criado após a colisão, assim como outras interações de estado final. A origem do efeito ridge em colisões $p p$ e $p A$ é uma questão em aberto atualmente. A busca por um melhor entendimento da origem desse efeito em sistemas pequenos e igualmente se estes sistemas também apresentam um comportamento "coletivo", é, ainda, um campo de pesquisa muito ativo nos dias de hoje.

Por fim, outra maneira de se estudar os efeitos coletivos que ocorrem em colisões hadrônicas é considerar as chamadas "assimetrias azimutais" mencionadas acima. No início dos anos 1990 foi proposto que assimetrias na distribuição de momento transversal de partículas produzidas em colisões ultra-relativísticas, que são medidas através da correlação angular de um ou mais pares de partículas, seriam uma assinatura 
não-ambígua do comportamento coletivo do sistema formado após a colisão de dois núcleos [178]. Neste caso, o caráter coletivo vem do fato de que as correlações estudadas devem envolver ao menos um par de partículas (que estão bem separados em pseudorapidez). Sendo assim, quanto maior o número de partículas correlacionadas, mais clara é a assinatura do comportamento coletivo. Algumas das várias assimetrias azimutais foram medidas em colisões $p p$ [152], $p(d) A[153,179-185]$ e $A A[138,139]$, parecendo indicar a presença de efeitos coletivos tanto em sistemas colisionais pequenos quanto àqueles formados somente por íons pesados.

A situação atual para este observável é bastante similar àquela do efeito ridge, porém apenas para colisões $p A$ : as abordagens hidrodinâmica e do CGC são capazes de descrever o mesmo conjunto de dados experimentais evocando mecanismos físicos completamente diferentes. Enquanto as assimetrias azimutais na abordagem hidrodinâmica têm origem nas interações de estado final, no CGC tais assimetrias estão presentes nos estágios iniciais da colisão e assume-se que não são destruídas devido à não formação do QGP.

Um estudo detalhado sobre as assimetrias azimutais em colisões $p A$ será desenvolvido no capítulo 5 desta tese onde, além de apresentar e discutir o formalismo matemático deste observável (de acordo com o quê é medido experimentalmente), apresenta-se um modelo capaz de gerar coeficientes de assimetria não nulos no estado inicial da colisão. Posteriormente, foca-se sua dependência com o momento transversal, $p_{T}$.

\subsection{O formalismo de dipolo de cor}

Próximo à região de saturação, $Q^{2}<Q_{s}^{2}$, a densidade de gluons é grande e esperase que as equações de evolução lineares da QCD sejam substituídas por equações de evolução não lineares. Neste regime, todavia, as informações contidas nas PDFs usuais não são suficientes para descrever de maneira satisfatória a dinâmica da interação 
entre projétil e alvo. Grosseiramente falando, as distribuições de pártons fornecem a probabilidade de encontrar um único párton no alvo e a interação com o projétil ocorre somente com esse dado párton. No entanto, no regime de altas densidades gluônicas é improvável que o projétil interaja uma única vez com o núcleo ao passar pelo mesmo. Neste caso, deve-se considerar múltiplas interações entre o projétil e os gluons que populam o alvo, que não são mais descritos pelas distribuições de gluons usuais, $x g\left(x, Q^{2}\right)$.

A descrição de múltiplas interações entre o projétil e o alvo em um espalhamento que ocorre a altas energias e pequenos valores de $x$ (portanto na presença de altas densidades partônicas no alvo) pode ser efetuada através do chamado "formalismo de dipolo de cor" [64-69], que será apresentado a seguir, e que é formulado em termos das chamadas "linhas de Wilson" na teoria efetiva do CGC.

Antes de apresentarmos este modelo faz-se necessário apresentar as coordenadas do cone de luz. Tais coordenadas são muitas vezes utilizadas na descrição de processos de espalhamento que ocorrem a altas energias e provam pequenos valores de $x$, que é exatamente o caso de interesse ${ }^{4}$. Especificamente, as coordenadas do cone de luz são importantes para a construção do modelo de dipolo de cor pois nele se faz necessário o acesso de componentes especificas do estado de Fock do projétil, como veremos nas próximas seções. Tais estados são autoestados da Hamiltoniana da QCD no gauge do cone de luz e podem ser calculados exatamente para alguns casos.

\subsubsection{Coordenadas do Cone de Luz}

No sistema de coordenadas do cone de luz um quadri-vetor qualquer tem a forma $l^{\mu}=\left(l^{+}, l^{-}, \mathbf{l}_{\perp}\right)$. As componentes de um quadri-vetor no cone de luz estão relacionadas

\footnotetext{
${ }^{4}$ Para uma descrição concisa da vantagem de se trabalhar no gauge do cone de luz e se utilizar as variáveis do cone de luz veja [186,187] e a seção 1.2 da referência [43].
} 
às componentes de um quadri-vetor usual $l^{\mu}=\left(l^{0}, l^{1}, l^{2}, l^{3}\right)$, através das relações

$$
l^{+}=\frac{l^{0}+l^{3}}{\sqrt{2}}, \quad l^{-}=\frac{l^{0}-l^{3}}{\sqrt{2}}, \quad \mathbf{1}_{\perp}=\left(l^{1}, l^{2}\right)
$$

Nessas coordenadas o eixo $z$ é escolhido para ser o eixo onde ocorre a colisão. As coordenadas do cone de luz possuem relações de transformação simples fazendo-se um boost na direção do eixo de colisão: $l^{+}=l^{+} e^{\psi}, l^{\prime-}=l e^{-\psi}$ e $\mathbf{l}_{\perp}^{\prime}=\mathbf{l}_{\perp}$. Aqui, $\psi=(1 / 2) \ln [(1+v) /(1-v)]$, onde $v$ é a velocidade da partícula. Nas coordenadas do cone de luz a aplicação de dois boosts seguidos, $\psi_{1}$ e $\psi_{2}$, tem como resultado final $\psi_{1}+\psi_{2}$. O quadri-momento de uma partícula de massa $m$ após a aplicação de um boost é

$$
p^{\mu}=\left(\frac{m}{\sqrt{2}} e^{\psi}, \frac{m}{\sqrt{2}} e^{-\psi}, \mathbf{0}_{\perp}\right)
$$

Pode-se ver claramente que um quadri-momento $\left(p^{\mu}\right)$ apresenta uma componente "grande" e uma componente "pequena" após a aplicação de uma transformação do tipo "boost" ao longo do eixo de colisão.

Por fim, a variável chamada "rapidez", $y$, tem a seguinte definição

$$
y=\frac{1}{2} \ln \left(\frac{p^{+}}{p^{-}}\right)=\frac{1}{2} \ln \left(\frac{E+p^{3}}{E-p^{3}}\right)
$$

onde a última igualdade é válida no sistema cartesiano de coordenadas.

A variável rapidez se transforma de maneira aditiva sob a aplicação de boosts, $y^{\prime}=y+\psi$.

\subsubsection{DIS no referêncial de repouso do alvo}

No capítulo anterior o processo de DIS foi apresentado no referêncial de momento infinito, onde no modelo a pártons um fóton virtual é espalhado por um próton que, por sua vez, consiste de quarks e principalmente gluons. Como pôde ser observado, a estrutura interna do próton em termos dos quarks e gluons era alterada a depender do 
valor de $x$ e $Q^{2}$ provados. O chamado "formalismo de dipolo de cor" [64-69,188] tem sua origem também no estudo de processos de DIS no referêncial de repouso do alvo, aqui considerado como um núcleo, $A$. Como antes, o processo ainda é descrito pela troca de um fóton virtual que, neste referêncial, carrega toda a energia da interação. Todavia, nesse formalismo, quando o fóton virtual prova o alvo em pequenos valores de $x$, ele prova, predominantemente, o campo de cor dos gluons. Certamente, o fóton não interage diretamente com os gluons do alvo, já que contém carga de cor nula. Neste caso, é necessário expandir o fóton virtual na base de estados de Fock em termos de suas possíveis flutuações quânticas ${ }^{5}$ :

$$
\left|\gamma^{*}\right\rangle=\Psi^{\gamma^{*} \rightarrow q \bar{q}}|q \bar{q}\rangle+\Psi^{\gamma^{*} \rightarrow q \bar{q} g}|q \bar{q} g\rangle+\ldots
$$

Na equação acima, $\Psi^{\gamma^{*} \rightarrow q \bar{q}}$ é a função de onda (do cone de luz) do processo onde o fóton virtual flutua num par $q \bar{q}$, que pode ser calculada perturbativamente via QED, e $|q \bar{q}\rangle$ denota o estado $q \bar{q}$, ou seja, um dipolo de cor. Sendo assim, $\left|\Psi^{\gamma^{*} \rightarrow q \bar{q}}\right|^{2}$ fornece a probabilidade do fóton virtual flutuar em um par $q \bar{q}$ e, posteriormente, do par $q \bar{q}$ aniquilar-se e se tornar um fóton. A interpretação é similar para os demais estados de Fock.

Em ordem mais baixa em teoria perturbativa, onde consideramos apenas o primeiro termo na equação (2.7), a altas energias e pequenos valores de $x$, o processo de espalhamento elétron-núcleo é descrito da seguinte maneira no formalismo de dipolo: o elétron incidente emite um fóton virtual, que por sua vez flutua em um par $q \bar{q}$, formando um dipolo de cor, que então se espalha, interagindo elasticamente com os campos de cor dos nucleons. Este processo esta representado diagramaticamente na Figura 2.7, onde $q^{\mu}\left(p^{\mu}\right)$ denota o quadri-momento do fóton (próton) e $z(1-z)$ denota a fração de momento carregada pelo quark (antiquark).

Olhando atentamente para a Figura 2.7 vê-se que o dipolo $q \bar{q}$ possui tamanho transversal $r_{T} \equiv r$ fixo. De fato, essa propriedade é verdadeira caso o tempo de

\footnotetext{
${ }^{5}$ Aqui exclui-se o caso onde o fóton não flutua em um estado de Fock de mais alta ordem pois, não existe interação entre projétil e alvo neste caso.
} 


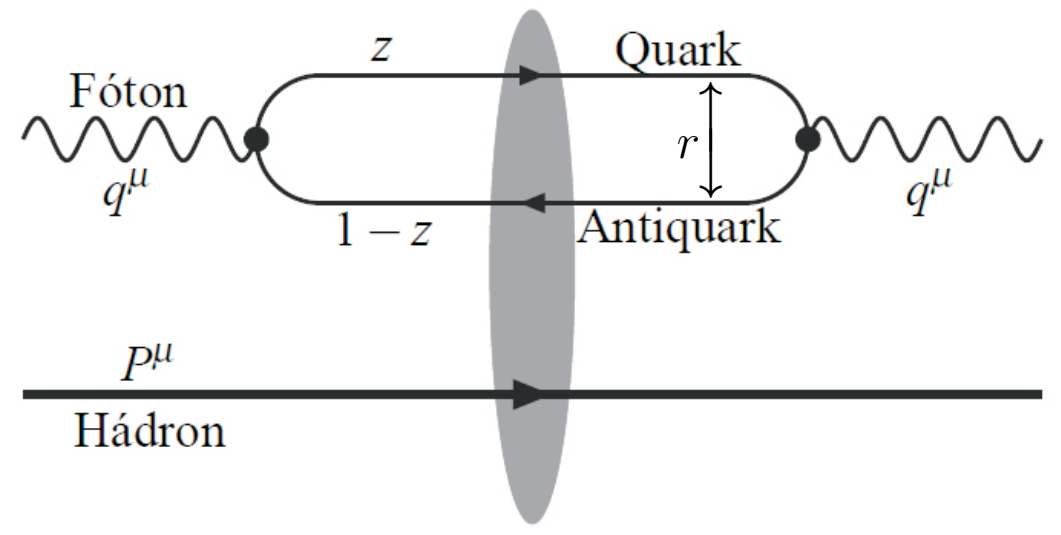

Figura 2.7: DIS no formalismo de dipolo de cor. O fóton virtual flutua em um par quark-antiquark que interage com o hádron alvo.

formação do dipolo (também conhecido como comprimento de coerência), $\tau_{\text {dip }}$, for muito maior que o tempo de interação entre o dipolo e o alvo, $\tau_{\text {int }}$, que é da ordem do raio do alvo $\left(\tau_{\text {int }} \sim R_{A}\right)$. Utilizando-se o princípio da incerteza é possível mostrar que $[41-43,58,106] \tau_{d i p}=1 /\left(2 m_{A} x\right)$, com $m_{A}$ sendo a massa do núcleo alvo e logo, tem-se que $\tau_{d i p} \gg \tau_{\text {int }}$, para $x \rightarrow 0$. Portanto, esse formalismo é ideal para o estudo de processos que ocorrem a altas energias e provam pequenos valores de fração de momento. Nestas condições o dipolo interage com o alvo sem ter seu tamanho alterado (o que é conhecido na literatura como "aproximação eiconal") e, portanto a matriz de espalhamento é diagonal em $r$. Por esse motivo, acredita-se que os dipolos de cor representem os graus de liberdade corretos em espalhamentos a altas energias.

Seguindo a discussão acima, no formalismo de dipolo a seção de choque total do espalhamento $\gamma^{*} A$ se fatoriza no produto do quadrado da função de onda do cone de luz, $\left|\Psi^{\gamma^{*} \rightarrow q \bar{q}}\right|^{2}$, representando o "splitting" do fóton num par $q \bar{q}$ e a seção de choque da interação entre o dipolo e o núcleo, $\sigma^{q \bar{q} A}(r, x)$ :

$$
\sigma^{\gamma^{*} A}\left(x, Q^{2}\right)=\int d z \int d^{2} r\left|\Psi^{\gamma^{*} \rightarrow q \bar{q}}\left(r, x, Q^{2}\right)\right|^{2} \sigma^{q \bar{q} A}(x, r)
$$

A vantagem de tal fatorização vem do fato de que o processo de espalhamento 
é separado em uma parte puramente eletromagnética, descrita pela QED, e outra contendo a dinâmica da QCD. Neste formalismo, $\sigma_{\text {dip }}(x, r) \equiv \sigma^{q \bar{q} A}(x, r)$ é quem carrega toda a informação sobre o alvo e a física das interações fortes e, portanto, sobre os efeitos da saturação. A quantidade $\sigma_{\text {dip }}(x, r)$ pode ser obtida a partir da amplitude de espalhamento elástico dipolo-hádron, $\mathcal{A}_{q \bar{q}}(x, r, \Delta)$, fazendo-se uso do teorema óptico $[106,188]$,

$$
\sigma_{d i p}(x, r)=\operatorname{Im} \mathcal{A}_{q \bar{q}}(x, r, \Delta=0)=\int d^{2} b \mathcal{N}\left(x, r, b \equiv b_{T}\right)=\sigma_{0} \mathcal{N}(x, r),
$$

onde $\Delta$ é o momento transversal perdido pelo hádron emergente e $\mathcal{N}(x, r, b)$ é parte imaginária da amplitude de espalhamento frontal dipolo-alvo, que está relacionada, de modo geral, com a matriz de espalhamento (ou matriz $S$ ) da seguinte maneira,

$$
\mathcal{N}(x, r, b)=1-S(x, r, b)
$$

Na obtenção da ultima igualdade em (2.9) ignorou-se a dependência com o parâmetro de impacto, $b$ e assumiu-se que a integral em $d^{2} b$ fornece um valor constante, $\sigma_{0}$, tratado como um parâmetro livre e, portanto, fixado através da análise de dados experimentais. Tal aproximação é válida para um núcleo infinitamente grande e homogêneo.

Infelizmente $\sigma_{d i p}(x, r)$ não é calculável a partir de primeiros princípios via teoria perturbativa uma vez que espalhamentos elásticos são processos de cunho não perturbativo $^{6}$. Desta maneira, na prática, utilizam-se modelos para $\mathcal{N}(x, r)$. Embora existam modelos para $\mathcal{N}(x, r, b)$ que não se utilizam da fatorização mencionada acima, sua motivação esta no fato de que a dependência das equações de evolução para as amplitudes de dipolo na variável $\vec{b}$ ainda não é bem estabelecida $[189,190]$ e, portanto, sua dependência é introduzida de maneira fenomenológica. Na próxima seção serão apresentados três modelos diferentes para a amplitude de espalhamento dipolo-alvo. Tais

\footnotetext{
${ }^{6}$ Isso pode ser entendido através do fato de que a transferência de momento em um espalhamento elástico é $\Delta=0$, e, portanto, intrinsecamente relacionado ao regime não perturbativo, onde abordagens perturbativas não são aplicáveis.
} 
modelos serão utilizados nos capítulos posteriores para estudar diferentes observáveis físicos de interesse nessa tese.

Nos trabalhos [64-69] os autores provaram que a fatorização apresentada na equação (2.8) é, de fato, válida e mostraram também que $\sigma_{\text {dip }}$ deve satisfazer duas propriedades devido à formação de dipolos com raio transversal muito pequeno ou muito grande. A primeira propriedade diz que no regime de pequenos valores do raio do dipolo $q \bar{q}$, $\sigma_{\text {dip }}$ é proporcional a $r^{2}$. Esta propriedade é conhecida como "transparência de cor", pois traduz o fato de que, se a distância entre o quark e o antiquark do dipolo é muito pequena, cor e anticor encontram-se praticamente sobrepostas e, portanto, a probabilidade de que o par interaja fortemente com o próton é também muito pequena, de forma que a seção de choque de dipolo tenda a zero quando o tamanho do dipolo aproxima-se de zero.

Todavia, no regime de grandes dipolos $(r \rightarrow \infty)$, devido à propriedade de confinamento da QCD, a seção de choque de dipolo deve saturar em um valor máximo $\sigma_{d i p} \sim \sigma_{0}=$ constante (ou seja, $\mathcal{N}(x, r)=1$ ), pois a medida que a distância entre o par quark e antiquark cresce, a seção de choque de dipolo aumenta, uma vez que as cargas de cor ficam mais afastadas e produzem um efeito maior ao interagir com o alvo (até saturar em um valor máximo).

Antes de tratarmos a respeito da importância de múltiplos espalhamentos, é importante mencionar que no formalismo de dipolo é possível utilizar $\sigma^{\gamma^{*} A}$ para calcular as funções de estrutura $F_{1,2}\left(x, Q^{2}\right)$. É simples mostrar que nesse formalismo são válidas as seguintes relações:

$$
\begin{aligned}
2 x F_{1}\left(x, Q^{2}\right) & =\frac{Q^{2}}{4 \pi^{2} \alpha_{\mathrm{QED}}} \sigma_{T}^{\gamma^{*} A}, \\
F_{2}\left(x, Q^{2}\right) & =\frac{Q^{2}}{4 \pi^{2} \alpha_{\mathrm{QED}}}\left(\sigma_{T}^{\gamma^{*} A}+\sigma_{L}^{\gamma^{*} A}\right),
\end{aligned}
$$

onde $\sigma_{T, L}^{\gamma^{*} A}$ correspondem às seções de choque envolvendo gluons com polarização transversal e longitudinal, respectivamente. 
O resultado do espalhamento entre um dipolo de cor e um único nucleon do alvo ${ }^{7}$ em mais baixa ordem em teoria perturbativa, a interação dipolo-nucleon ocorre via troca de dois gluons $[64-69,106]$ e o resultado para a seção de choque de interação dipolo-alvo é, neste caso, dado por

$$
\sigma_{d i p} \approx \frac{2 \pi \alpha_{s}^{2} C_{F}}{N_{c}} r^{2} \ln \left(\frac{1}{r^{2} \Lambda^{2}}\right)
$$

com $C_{F}=\left(N_{c}^{2}-1\right) / 2 N_{c}$ e $\Lambda$ sendo um corte na região do infravermelho.

Na obtenção da equação acima assume-se que o dipolo possui tamanho pequeno, muito menor que o tamanho do nucleon, $r \ll 1 / \Lambda$ e, considerando-se a situação mais simples, onde o nucleon é composto apenas de um quark de valência, tem-se que a distribuição de gluons de um nucleon é [191],

$$
x g\left(x, Q^{2}\right)=\frac{2 \alpha_{s} C_{F}}{\pi} \ln \left(\frac{Q^{2}}{\Lambda^{2}}\right)
$$

de modo que

$$
\sigma_{d i p} \approx \frac{\pi^{2} \alpha_{s}}{N_{c}} r^{2} x g\left(x, Q^{2}=1 / r^{2}\right)
$$

A equação (2.15), válida para o espalhamento $\gamma^{*} A$, pode ser generalizada para o caso do espalhamento entre um dipolo e um nucleon apenas trocando-se a distribuição de gluons, $x g\left(x, Q^{2}\right) \rightarrow x g_{N}\left(x, Q^{2}\right)$, onde $x g_{N}\left(x, Q^{2}\right)$ denota a distribuição de gluons de um nucleon. Como visto na seção 1.3.1, onde estudou-se o limite de duplo logaritmo dominante das equações DGLAP, a seção de choque de dipolo viola a unitaridade para grandes valores de $x g_{N}\left(x, Q^{2}=1 / r^{2}\right)$, quando a altas energias e grandes densidades partônicas, a probabilidade de ocorrer múltiplos espalhamentos se torna grande, devendo ser considerada. A inclusão de múltiplos espalhamentos entre projétil e (núcleo) alvo para o caso da QCD foi desenvolvida na referência [191] e uma derivação mais simples (porém bem menos formal) pode ser encontrada na referência [58].

\footnotetext{
${ }^{7}$ Enquanto aqui focaremos nos resultados e suas interpretações, uma derivação detalhada dos resultados aqui mencionados pode ser encontrada em [106].
} 
Aqui vamos nos restringir a tecer o seguinte comentário: utilizando-se a aproximação de Glauber [192,193] (a ser desenvolvida na seção 3.3), que consiste em considerar um núcleo grande e diluído, com $A \gg 1$ nucleons não correlacionados, ou seja, independentes, e se mantendo em mais baixa ordem em teoria perturbativa, pode-se mostrar que o espalhamento do projétil com dois nucleons se fatoriza no produto de dois espalhamentos independentes do dipolo com um único nucleon. Neste caso, a seção de choque de interação de um dipolo com um número arbitrário de nucleons pode ser obtida via iteração.

Finalmente, a expressão resultante para a amplitude de espalhamento frontal ${ }^{8}$ da interação entre um dipolo de cor e um núcleo incluindo múltiplos espalhamentos é dada por,

$$
\mathcal{N}(r, y=0)=1-\exp \left[-\frac{\alpha_{s} \pi^{2}}{2 N_{c}} \rho T(b) r^{2} x g_{N}\left(x, 1 / r^{2}\right)\right]
$$

onde $\rho$ representa a densidade de nucleons no núcleo $\left(\rho=A /\left[(4 / 3) \pi R_{A}^{3}\right]\right.$, para um núcleo esférico de raio $R_{A}$ ), e $T(b)$ é a função de perfil nuclear, que é igual ao comprimento do meio nuclear a um dado parâmetro de impacto $b\left(T(b)=\sqrt{R_{A}^{2}-b^{2}}\right)$.

Esta expressão é conhecida como formula de Glauber-Mueller [106, 191-193] pois apresenta uma generalização do modelo de Glauber de múltiplas colisões independentes no núcleo para o caso da QCD.

Quando o produto do tamanho do dipolo pela densidade de nucleons no núcleo é pequeno, $\rho r \ll 1$, a amplitude de dipolo é aproximadamente igual a

$$
\mathcal{N}(r, y=0) \approx \frac{\alpha_{s} \pi^{2}}{2 N_{c}} \rho T(b) r^{2} x g_{N}\left(x, 1 / r^{2}\right)
$$

Utilizando-se a expressão (2.14) e definindo-se a escala de saturação como

$$
Q_{s}^{2}(b)=\frac{4 \pi \alpha_{s}^{2} C_{F}}{N_{c}} \rho T(b),
$$

\footnotetext{
${ }^{8}$ Neste caso, é necessário efetuar a integral no parâmetro de impacto para se obter a seção de choque dipolo-alvo.
} 
a amplitude de espalhamento dipolo-núcleo se torna

$$
\mathcal{N}(r, y=0)=1-\exp \left[-\frac{r^{2} Q_{s}^{2}(b)}{4} \ln \left(\frac{1}{r \Lambda}\right)\right]
$$

Se analisarmos o resultado desta última equação, como ponto de partida, já que $\mathcal{N} \leq 1$ para todos os valores (perturbativos) de $r$, é possível perceber que o problema da violação da unitaridade encontrado anteriormente no caso do espalhamento do dipolo com um único nucleon (vide equação (2.15)) deixa de existir. Sendo assim, para que a seção de choque dipolo-alvo não viole a unitaridade se faz mandatório a inclusão de múltiplos espalhamentos!

Nesse ponto é interessante analisar o comportamento da amplitude de espalhamento dipolo-alvo da equação (2.19), que é apresentado (de maneira esquemática) na Figura 2.8 como função de $r$. Para o caso de pequenos dipolos, onde $r \ll Q_{s}^{-1}$, tem-se que $\mathcal{N}(r, y=0) \propto r^{2}$, que é a propriedade de transparência de cor, como apontado anteriormente. Por outro lado, para $r \gtrsim Q_{s}^{-1}$, a amplitude de espalhamento dipolo-alvo satura em um valor máximo, $\mathcal{N}=1$. Este resultado corresponde ao chamado "limite de disco negro" (acima mencionado), já que $\sigma_{d i p}=2 \int d^{2} b \mathcal{N}(x, y, b)=2 \pi R_{A}^{2}$, é o resultado obtido efetuando-se a integral no parâmetro de impacto quando $\mathcal{N}(x, y, b)=1$ em todo o espaço de fase, e se constitui, exatamente, na seção de choque de uma partícula espalhada por uma esfera negra.

Na referência [191] foi mostrado que a saturação da amplitude de espalhamento de dipolo se traduz na saturação da distribuição de pártons no núcleo. Se assim o acreditarmos esse fato pode ser visto de maneira simples através da equação (2.12), onde $F_{2} \sim q(x)+\bar{q}(x) \sim \sigma_{\text {tot }}^{\gamma^{*} A}$. Ou seja, uma vez que a amplitude de espalhamento dipolo-alvo satura, o que corresponde a $\mathcal{N}(x, r, b)=1$ na equação $(2.8), \sigma_{\text {tot }}^{\gamma^{*} A}$ atinge seu valor máximo, assim como a função de estrutura que, por sua vez, relaciona-se com a distribuição de pártons no núcleo.

Nesse ponto é importante denotar o seguinte fato: uma vez que $T(b) \sim A^{1 / 3}$, $Q_{s}^{2}$ também cresce com $A^{1 / 3}$, como discutido anteriormente (vide equação (2.1)). É 


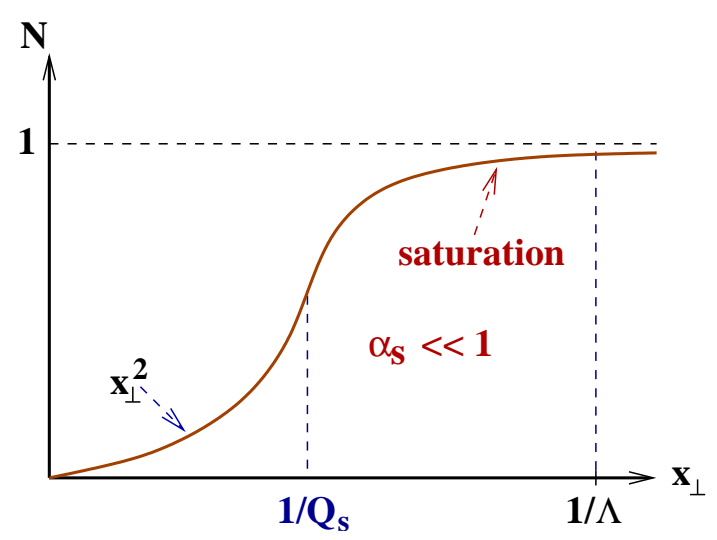

Figura 2.8: Amplitude de espalhamento frontal dipolo-alvo, $\mathcal{N}\left(x, r \equiv x_{\perp}\right)$ como função da separação transversa entre quark e anti-quark, $r$ ( $\equiv x_{\perp}$ nesta figura), que formam um dipolo de cor. Figura extraída de [58].

possível perceber que para um núcleo muito grande, a escala de saturação se torna muito maior que a escala da $\mathrm{QCD}, \Lambda_{Q C D}$. Uma vez que a transição entre os comportamentos $\mathcal{N} \sim r^{2}$ e $\mathcal{N}=1$ ocorre na região onde $r \sim Q_{s}^{-1}$ (vide Figura 2.8), quando $Q_{s} \ll \Lambda_{Q C D}$ a transição para o limite de disco negro ocorre para pequenos valores de $r$ (que correspondem a grandes valores de momento transversal) e, portanto, dentro do regime perturbativo. Essa análise justifica a aproximação considerada na construção da amplitude de espalhamento dipolo-núcleo, onde as múltiplas interações dipolo-nucleon foram descritas via teoria perturbativa (troca de dois gluons) e não por algum mecanismo não perturbativo.

O modelo de dipolo desenvolvido até aqui diz respeito às interações do tipo DIS, que são mediadas via troca de um fóton virtual, $\gamma^{*}$. Neste caso, o dipolo de cor $q \bar{q}$ surge naturalmente como uma flutuação quântica do fóton virtual. Todavia, nos capítulos seguintes trabalharemos com colisões hadrônicas, onde o processo não é mediado pela troca de um fóton virtual. Sendo assim, é importante descrevermos como as amplitudes de espalhamento dipolo-alvo são construídas nesse tipo de colisão e, porquanto, fazse necessário apresentar alguns detalhes referentes à formulação da teoria efetiva do CGC.

Em primeiro lugar, essa teoria efetiva é construída assumindo-se a aproximação eiconal, isto é, na aproximação onde o projétil (digamos um quark) atravessa o alvo 
sofrendo múltiplos espalhamentos porém sem ter sua trajetória desviada, ele se propaga em linha reta ao passar pelo alvo.

Tal formalismo foi construído utilizando-se as chamadas "linhas de Wilson" [41$58,194], V\left(x_{\perp}\right)$, definidas por

$$
V\left(x_{\perp}\right)=\mathcal{P} \exp \left[i g \int_{-\infty}^{\infty} d x^{-} A^{+a}\left(x^{-}, x_{\perp}\right) t^{a}\right]
$$

onde $x_{\perp}$ é a coordenada transversal do quark, $a$ é o índice de cor, $t^{a}$ são os geradores do grupo de cor $(S U(3)$ para a QCD) na representação fundamental e o símbolo $\mathcal{P}$ denota o ordenamento dos campos $A^{+}\left(x^{-}, x_{\perp}\right)=A^{+a}(\vec{x}) t^{a}$, que são matrizes $N_{c} \times N_{c}$, no expoente da direita para a esquerda da expressão acima com o acréscimo do valor de $x^{-}$.

Nesta equação $g$ representa o acoplamento forte, relacionado à constante de acoplamento forte através da equação (1.31). Fisicamente, a equação acima representa o efeito acumulado do campo $A^{\mu}$ em uma partícula (um fermion) passando por ele ao longo de uma linha reta na posição $x_{\perp}$. A interação entre a partícula e o campo contribui com um fator $t^{a}$ e expandindo-se a exponencial na equação (2.20) tem-se que

$$
\begin{aligned}
V\left(x_{\perp}\right) & =1-i g \int_{-\infty}^{\infty} d x^{-} A^{+}\left(x^{-}, x_{\perp}\right) \\
& +\frac{(i g)^{2}}{2} \int_{-\infty}^{\infty} d x^{-} \int_{-\infty}^{x^{-}} d x^{\prime-} \mathcal{P}\left[A^{+}\left(x^{-}, x_{\perp}\right) A^{+}\left(x^{\prime}, x_{\perp}\right)\right]-\ldots
\end{aligned}
$$

A partir dessa equação é possível observar que a equação (2.20) "ressoma" múltiplos espalhamentos. Por outro lado, se na equação (2.20) o gerador do grupo $S U(3)$ estiver na representação adjunta (note-se que o gerador do grupo de cor para o férmion acima encontrava-se na representação fundamental do grupo $S U(3)$ da QCD!), denotado por $T^{a}$, a linha de Wilson (que passa a ser denotada por $U\left(x_{\perp}\right)$ ) representará, simplesmente, a superposição de múltiplas interações entre o gluon que se propaga e o campo gluônico (do alvo) que atravessa. Alternativamente, pode-se escrever os campos gluônicos $\left(A^{\mu}\right)$ em termos das linhas de Wilson $U\left(x^{-}, x_{\perp}\right)$. Todavia, como neste 
momento estamos interessados em apresentar como a amplitude de espalhamento de dipolo se relaciona aos espalhamentos hadrônicos, continuaremos trabalhando com as linhas de Wilson.

Essas linhas se tornam particularmente interessantes quando as escrevemos como "loops de Wilson". O traço de produtos de linhas de Wilson nesse caso formam uma trajetória fechada e, somada à operação de traço, conduzem ao resultado de que os loops de Wilson são automaticamente invariantes de Gauge [195]. De fato, qualquer quantidade invariante de Gauge na teoria pode ser escrita em termos de loops de Wilson [195].

A altas energias, a interação entre dipolo-hádron no DIS ocorre para um tamanho fixo do dipolo $q \bar{q}$, onde essa interação pode ser tratada na aproximação eiconal na teoria efetiva do CGC e qualquer mudança de trajetória do dipolo devido à interação com o campo gluônico do alvo pode ser ignorada. Pode ser mostrado [41-44,48-58] (veja a seção 5.1.3 de [106] para uma discussão mais completa) que a matriz $S$ para um espalhamento dipolo-hádron é obtido calculando-se a correlação de duas linhas de Wilson, ou simplesmente uma "função de dois pontos",

$$
S\left(x_{\perp}, y_{\perp}\right) \equiv \frac{1}{N_{c}}\left\langle\operatorname{Tr}\left(V^{\dagger}\left(x_{\perp}\right) V\left(y_{\perp}\right)\right)\right\rangle
$$

onde "Tr" indica a operação de traço (aqui ele surge do fato do dipolo $q \bar{q}$ ser um estado de cor neutra (branca)).

O "tamanho" do dipolo é dado pela distância entre quark e antiquark, $r_{\perp} \equiv r=$ $\left|x_{\perp}-y_{\perp}\right|$. O simbolo $\langle\ldots\rangle$ indica a média em todas as configurações de cor do alvo ${ }^{9}$. Uma vez determinada a forma da matriz $S$ de um espalhamento dipolo-hádron em um processo do tipo DIS na teoria efetiva do CGC, pode-se facilmente mostrar como essa quantidade aparece em um espalhamento hadrônico. Para isso, deixe-nos considerar agora um espalhamento quark-hádron, onde um hádron colidente é altamente populado

\footnotetext{
${ }^{9}$ A cada espalhamento, o alvo prova uma configuração de cor particular do alvo, portanto deve-se efetuar a média sobre todas as configurações de cores do alvo de modo a calcular um dado observável físico.
} 
por gluons, ou seja, encontra-se no regime denso, onde espera-se a formação do CGC.

A altas energias, e na aproximação eiconal, o quark se propaga em linha reta e a amplitude de espalhamento correspondendo a um espalhamento elástico com momento inicial $p$ e final $q$ é $[196,197]$

$$
\langle\text { out, } q| \text { in, } p\rangle \equiv \bar{u}(q) \tau(q, p) u(p)
$$

onde $u(p)$ e $\bar{u}(q)$ são os espinores do quark nos estados inicial e final, e

$$
\tau(q, p)=2 \pi \delta\left(p^{-}-q^{-}\right) \gamma^{-} \int d^{2} x_{\perp}\left[V\left(x_{\perp}\right)-1\right] e^{i(\vec{p}-\vec{q}) \cdot x_{\perp}}
$$

com $V\left(x_{\perp}\right)$ dado pela equação $(2.20)$.

Quadrando-se a amplitude de espalhamento dada pela equação (2.23), obtém-se a seção de choque total ${ }^{10}[196,197]$

$\frac{d \sigma}{d^{2} b d^{2} k}=\frac{1}{(2 \pi)^{2}} \int d^{2} r e^{-i \vec{k} \cdot \vec{r}}\left(\left\langle\frac{1}{N_{c}} \operatorname{Tr}\left(W(\vec{r}, \vec{b})-V(\vec{b}-\vec{r} / 2)-V^{\dagger}(\vec{b}+\vec{r} / 2)\right)\right\rangle+1\right)$.

Aqui $b_{\perp}=\left(x_{\perp}+y_{\perp}\right) / 2$ é o parâmetro de impacto da colisão, $r_{\perp} \equiv r=x_{\perp}-y_{\perp}$, $W(\vec{r}, \vec{b})$ é um loop de Wilson com de largura $r=|\vec{r}|$. No Gauge covariante, $\partial_{\mu} A^{\mu}=$ 0 , a quantidade $W(\vec{r}, \vec{b})=V^{\dagger}(\vec{b}+\vec{r} / 2) V(\vec{b}-\vec{r} / 2)$ é comummente referida como a distribuição não integrada de gluons [64-69]. A expressão entre parênteses corresponde à matriz $S$ de um dipolo de tamanho e orientação dados por $\vec{r}$.

À primeira vista a expressão (2.25) não corresponde à equação (2.22) já que contém mais termos. Integrando-se a equação (2.25) em $d^{2} k_{T}$ tem-se que os termos adicionais, isto é além de $W(\vec{r}, \vec{b})$, são proporcionais a função delta de Dirac (com argumento $r$ ), $\delta(r)$, e, portanto, são importantes apenas quando o observável de interesse é a seção de choque total, já que, neste caso, deve-se incluir as contribuições dos espalhamentos

\footnotetext{
${ }^{10}$ Uma vez que nosso interesse principal é indicar como a matriz $S$ de um espalhamento dipolohádron aparece na seção de choque hadrônica, vamos omitir as etapas intermediárias na obtenção da equação (2.25). Uma derivação detalhada desta equação pode ser encontrada na seção 3.2 da referência [198].
} 
elásticos, onde a troca de momento é nula [197].

Como em colisões hadrônicas estamos interessados, na imensa maioria das vezes, em processos que ocorrem com momento transversal não nulo, estes termos deixam de ser importantes e, finalmente, tem-se que

$$
\frac{d \sigma}{d^{2} b d^{2} k}=\frac{d N}{d^{2} k}=\frac{1}{(2 \pi)^{2}} \int d^{2} r e^{-i \vec{k} \cdot \vec{r}} \frac{1}{N_{c}}\left\langle\operatorname{tr}\left(V^{\dagger}(\vec{b}+\vec{r} / 2) V(\vec{b}-\vec{r} / 2)\right)\right\rangle
$$

onde vê-se claramente que a expressão resultante é exatamente a transformada de Fourier da matriz $S$ de um dipolo de cor.

No processo de DIS é interessante trabalhar na representação do espaço de configurações onde, em colisões hadrônicas, utilizam-se os ingredientes necessários para o espaço dos momentos.

A seguir descrevemos três modelos para a amplitude de espalhamento dipolo-alvo que utilizaremos nos cálculos do capítulo 4 desta tese. Um quarto modelo de dipolo será apresentado e utilizado em cálculos analíticos no capítulo 5.

\subsubsection{O modelo GBW}

Em 1999, Golec-Biernat e Wüsthoff (GBW) [113,121] propuseram um primeiro modelo fenomenológico para a amplitude de espalhamento frontal dipolo-alvo. De modo geral é assumido que a amplitude de espalhamento pode ser modelada segundo uma função eiconal do tipo [35-43, 57]:

$$
\mathcal{N}(x, r)=1-\exp \left[-\frac{1}{4}\left(r^{2} Q_{s}^{2}\right)^{\gamma\left(x, r^{2}\right)}\right]
$$

onde o expoente $\gamma$ é usualmente referido como a "dimensão anômala" da distribuição de gluons no alvo e

$$
Q_{s}^{2}(x)=Q_{0}^{2}\left(\frac{x_{0}}{x}\right)^{\lambda}
$$

é a escala de saturação, já mencionada anteriormente, uma escala de momento que es- 
tabelece o regime em que os efeitos de saturação passam a ter importância significativa no processo de espalhamento e, ao que tudo indicam os mais recentes experimentos, não deveriam mais ser desprezados.

Na equação acima tem-se que $Q_{0}^{2}=1.0 \mathrm{GeV}^{2}, x_{0}=3.0 \cdot 10^{-4}$ e $\lambda=0.288[113,121]$. Neste modelo considera-se $\gamma\left(x, r^{2}\right)_{G B W}=1$ e os parâmetros $\sigma_{0}, \lambda$ e $x_{0}$ fixados de modo a tornar possível a descrição dos resultados experimentais do HERA . A dependência de $\mathcal{N}(x, r)$, apenas através da combinação $r^{2} Q_{s}^{2}(x)$, implica que a seção de choque do DIS nesse modelo depende somente da combinação $Q_{s}^{2}(x) / Q^{2}=1 / \tau[113,121]$, satisfazendo a propriedade do chamado "scaling geométrico". Uma inspeção rápida da equação (2.27), com $\gamma\left(x, r^{2}\right)=\gamma\left(x, r^{2}\right)_{G B W}$, mostra que o modelo GBW possui as propriedades comentadas anteriormente quando ocorre a formação de um dipolo com raio transversal muito pequeno ou muito grande.

Embora a parametrização GBW descreva bem os dados de HERA [22-25, 29-31] anteriores ao ano 2000, essa descrição deixa de ser boa para os dados obtidos a partir desse ano por conta de uma maior precisão na obtenção dos dados experimentais. Esta limitação vem do fato de que este modelo falha ao descrever a violação do scaling de Bjorken: a grandes valores de $Q^{2}$ o ajuste aos dados da função de estrutura do próton, $F_{2}$, não se iguala àquela obtida utilizando-se as equações DGLAP, que, por sua vez, fornecem justamente o quão forte é a violação do scaling de Bjorken neste observável.

A forma funcional do modelo GBW vem apenas de uma aproximação da QCD não linear [199], uma vez que essa parametrização se assemelha a soluções numéricas da equação BK [200-203], que é uma equação de evolução não linear obtida dentro da teoria efetiva do CGC. Uma discussão qualitativa mais detalhada sobre as equações de evolução será apresentada na próxima seção.

\subsubsection{Os modelos BUW e DHJ}

A principal diferença entre os diversos modelos encontrados na literatura vem 
do comportamento predito pela dimensão anômala, responsável por determinar quão rápido ocorre a transição do regime de saturação (não linear) para o regime diluído (linear).

Na referência [204] Dumitru, Hayashigaki e Jalilian-Marian (DHJ) propuseram a seguinte parametrização para a dimensão anômala,

$$
\gamma(x, r)_{D H J}=\gamma_{s}+\left(1-\gamma_{s}\right) \frac{\left|\log \left(1 / r^{2} Q_{s}^{2}\right)\right|}{\lambda y+d \sqrt{y}+\left|\log \left(1 / r^{2} Q_{s}^{2}\right)\right|},
$$

onde $\gamma_{s}=0.628$, que é o valor obtido para a dimensão anômala a partir da equação BFKL em ordem dominante, $Q_{s}^{2}=A^{1 / 3} Q_{0}^{2}\left(x_{0} / x_{2}\right)^{\lambda}, Q_{0}^{2}=1.0 \mathrm{GeV}^{2}, x_{0}=3.0 \cdot 10^{-4}$, $\lambda=0.288$, e $d=1.2$.

Este modelo foi proposto para descrever o espectro de momento transversal medido em colisões $d A u$ na região frontal na maior energia do RHIC, $\sqrt{s}=200 \mathrm{GeV}$, levando em consideração violações do scaling geométrico. Como dito anteriormente, tais violações são caracterizadas pelos termos dependentes da rapidez do alvo $\left(y=\log \left(1 / x_{2}\right)\right)$ na equação acima, onde o parâmetro $d$ controla a intensidade de termos subdominantes em $y$.

Por outro lado, na referência [205] os autores Boer, Utermann e Wessels (BUW) propuseram a seguinte parametrização para a dimensão anômala:

$$
\gamma\left(\omega=q_{T} / Q_{s}\right)_{B U W}=\gamma_{s}+\left(1-\gamma_{s}\right) \frac{\left(\omega^{a}-1\right)}{\left(\omega^{a}-1\right)+b}
$$

onde $q_{T}$ representa o momento transversal do párton espalhado. Os parâmetros deste modelo, $\gamma_{s}=0.628, a=2.82$ e $b=168$ foram fixados de modo a descrever o espectro de momento transversal de hádrons produzidos em colisões $p p$ e $d A u$ nas energias do RHIC, $\sqrt{s}=62,4 \mathrm{GeV}$ e $200 \mathrm{GeV}$ [205]. Com esses parâmetros e desconsiderando-se a massa dos quarks leves, este modelo também foi capaz de descrever os dados da seção de choque $\sigma_{\gamma^{*} p}$ colisões ep no HERA.

Uma importante característica desta parametrização para a dimensão anômala é a 
realização explícita do scaling geométrico que é predito por soluções das equações de evolução da teoria efetiva do CGC no regime de altíssimas energias.

Uma vez que os mesmos dados experimentais, a saber, o espectro de momento transversal de hádrons medidos nas energias do RHIC, são descritos pelos modelos DHJ e BUW [204,205], não é possível dizer se tais dados experimentais apresentam ou não violações do scaling geométrico. Tais violações são esperadas na região de altos momentos transversais, onde a dimensão anômala tende à unidade e o comportamento predito pelas equações DGLAP deveria ser recuperado. Em princípio, espera-se que avanços com relação a essa questão possam ser obtidos ao se considerar as distribuições de momento transversais de hádrons produzidos nas energias do LHC, uma vez que, nesse caso, os dados experimentais são obtidos numa região maior de momento transversal quando comparados aos dados do RHIC. Essa questão será abordada na seção 4.4 desta tese.

\subsection{A saturação de gluons segundo as equações de evolução}

Muito do desenvolvimento teórico da física relacionada a pequenos valores de $x$, ou seja, a altas energias, aconteceu e ainda acontece em torno da procura de soluções das equações de evolução que se propõem a descrever como os pártons evoluem no interior de um hádron no regime de saturação. Como pode ser visto na Figura 2.9, no regime de saturação a evolução da distribuição de gluons se torna naturalmente não linear: no instante inicial os hádrons colidentes emitem gluons que originam duas diferentes cascatas através de múltiplos processos de emissão e, quando o processo de recombinação de gluons se torna favorável, dois ou mais gluons de diferentes cascatas podem se recombinar fazendo com que a dinâmica se torne não linear.

Embora a discussão acerca da Figura 2.9 seja qualitativa ela possui conexão com 


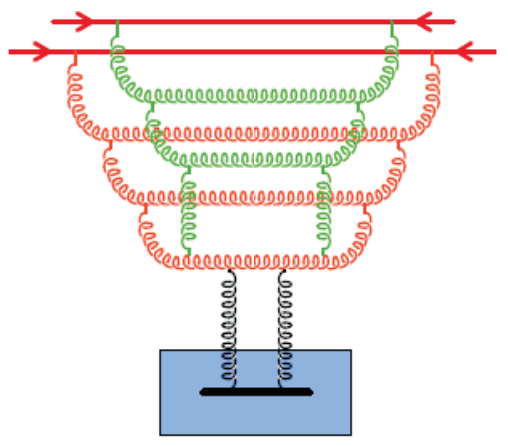

Figura 2.9: Esquema da evolução da distribuição de gluons incorporando o efeito de recombinação durante uma colisão a altas energias. Extraído de [41].

as equações de evolução que consideram o fenômeno da recombinação de gluons. A teoria colinear padrão da QCD não considera processos dessa natureza. Supõe-se que sua inclusão, numa primeira aproximação, poderia ser realizada a partir do cálculo de correções de mais altas ordens para as equações DGLAP. Os primeiros termos dessas correções, que envolvem uma série de diagramas de Feynman, foram calculados por Gribov, Levin e Ryskin [33] e Mueller e Qiu [34], e deram origem às equações hoje conhecidas por GLRMQ:

$$
\begin{aligned}
\frac{\partial x g\left(x, Q^{2}\right)}{\partial \log Q^{2}} & =\left.\frac{\partial x g\left(x, Q^{2}\right)}{\left.\partial \log Q^{2}\right)}\right|_{\text {DGLAP }}-\frac{9 \pi}{2} \frac{\alpha_{s}^{2}}{Q^{2}} \int_{x}^{1} \frac{d y}{y} y^{2} G^{(2)}\left(y, Q^{2}\right), \\
\frac{\partial x \bar{q}\left(x, Q^{2}\right)}{\partial \log Q^{2}} & \left.\approx \frac{\partial x \bar{q}\left(x, Q^{2}\right)}{\left.\partial \log Q^{2}\right)}\right|_{\text {DGLAP }}-\frac{3 \pi}{20} \frac{\alpha_{s}^{2}}{Q^{2}} x^{2} G^{(2)}\left(x, Q^{2}\right)
\end{aligned}
$$

onde $G^{(2)}\left(x, Q^{2}\right)$ representa a correção devida aos processos de fusão de gluons.

As equações de evolução para as distribuições de quarks de valência ficam inalteradas após a adição dessas correções e correções de mais altas ordens para a equação de evolução das distribuições de quarks do mar são desprezadas. O sinal negativo do termo não linear vem do fato de que a inclusão dos diagramas de recombinação de gluons (calculados em [33,34]) claramente devem levar à diminuição da distribuição de gluons.

Devido à dificuldade em determinar $G^{(2)}\left(x, Q^{2}\right)$, Mueller e Qiu estudaram dife- 
rentes fontes de contribuição para $G^{(2)}\left(x, Q^{2}\right)$ [34]. Valendo-se de uma abordagem fenomenológica, um dos casos estudados é aquele em que $G^{(2)}\left(x, Q^{2}\right)$ tem como contribuição processos caracterizados por cascatas independentes de gluons, iniciadas por diferentes quarks de valência ${ }^{11}$. Nesta situação supõe-se que cada um dos três quarks de valência movem-se livremente dentro de uma esfera de raio $R$ e, de maneira qualitativa, $G^{(2)}\left(x, Q^{2}\right)$ podendo ser modelado como [34]

$$
x^{2} G^{(2)}\left(x, Q^{2}\right)=\frac{2}{3} \frac{\left[x g\left(x, Q^{2}\right)\right]^{2}}{\pi R^{2}} .
$$

Quando os dois termos do lado direito da equação (2.31) possuem contribuições comparáveis, isto é, quando $\partial x g\left(x, Q^{2}\right) / \partial \log Q^{2} \approx 0$, os efeitos da recombinação de gluons se tornam importantes fazendo com que a distribuição de gluons sature em um dado valor. Utilizando-se essa equação e a condição $\partial x g\left(x, Q^{2}\right) / \partial \log Q^{2}=0$ na equação (2.31), tem-se que o valor máximo para a distribuição de gluons dentro de um próton é dada por

$$
x g\left(x, Q^{2}\right) \approx \frac{R^{2} Q^{2}}{\pi \alpha_{s}\left(Q^{2}\right)} \approx \frac{25,76}{\pi \alpha_{s}\left(Q^{2}\right)} Q^{2}
$$

onde $R=1 \mathrm{fm}$ e $Q$ é dado em GeV.

A condição representada pela equação (2.34) só pode ser alcançada para valores muito grandes de $1 / x$ ou para valores pequenos de $Q^{2}$. Fora desta região o efeito do termo não linear é desprezível, como esperado.

A despeito das equações GLRMQ ilustrarem o fato de que a inclusão dos efeitos de recombinação nas equações de evolução da QCD faz com que elas se tornem não lineares, percebe-se através desse fato (e também da equação (2.34)) que uma abordagem focada na inclusão de correções não lineares às equações DGLAP não é capaz de levar em conta corretamente os efeitos de saturação, uma vez que sua evolução é importante para altos valores de $Q^{2}$, região onde tais efeitos não são importantes. Outro ponto a ser destacado, que limita o uso da equação GLRMQ na vizinhança da região onde os efeitos de recombinação começam a se tornar importantes, é o fato da necessidade de

\footnotetext{
${ }^{11}$ Esta é exatamente a situação encontrada na Figura 2.9 para dois quarks de valência.
} 
termos de adicionar cada vez mais correções de mais alta ordem, à medida em que $Q^{2}$ vai diminuindo, o que se torna extremamente complicado.

Sendo assim, o cenário mais indicado para o estudo dos fenômenos não lineares implica em se levar em consideração as equações de evolução na variável $x$, mantendose um valor pequeno ou moderado para $Q^{2}$. Em consequência as equações de evolução no regime de saturação se promovem a generalizações da equação BFKL, apresentada no capítulo anterior, uma vez que esta considera apenas os processos de emissão de partículas.

O trabalho pioneiro de Gribov, Levin e Ryskin [33] gerou uma série de outros trabalhos [35-58] cujo objetivo foi desenvolver uma teoria mais sólida para a descrição de sistemas altamente densos, onde o fenômeno da saturação de pártons é imprescindível. Outras equações de evolução foram derivadas levando-se em consideração os processos de fusão de gluons e/ou a inclusão de múltiplos espalhamentos com o intuito de tentar descrever de uma maneira mais adequada a evolução da função de onda hadrônica em colisões a altas energias.
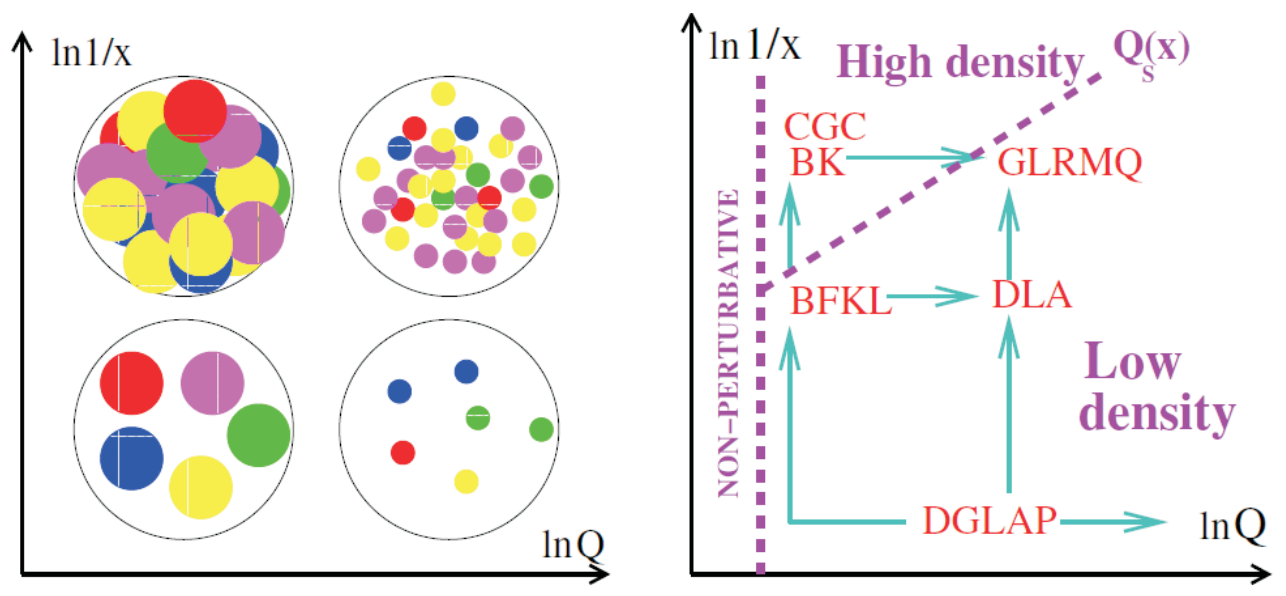

Figura 2.10: Esquema das equações de evolução no plano $\ln (1 / x)-\ln Q$ com a respectiva imagem pictórica a nível partônico. Extraído de [206].

Hoje nosso entendimento sobre esses diferentes regimes, segundo a ótica das equações de evolução, pode ser representada pela Figura 2.10. Na região de pequenos valores de $Q^{2}$ existe a região de confinamento, onde os métodos de pQCD não são aplicáveis. Para 
valores grandes de $Q^{2}$ e valores não tão grandes de $1 / x$ encontramos a região de baixa densidade partônica, onde as equações de evolução usuais, DGLAP e BFKL, podem ser utilizadas e fornecem resultados confiáveis. Para grandes valores de $1 / x$ e $\ln Q^{2}$ adentramos no regime de DLLA onde se encontra a primeira equação de evolução não linear proposta, a equação GLRMQ, que é valida apenas na região próxima à escala de saturação. Finalmente no regime de altas densidades, separado do regime de baixas densidades por uma linha que define justamente a escala de saturação, $Q_{s}$, encontra-se uma generalização da equação BFKL, a equação Balitsky-Kovchegov (BK) [44-47] e a equação desenvolvida por Jalilian-Marian, Iancu, McLerran, Weigert, Leonidov e Kovner (JIMWLK) [48-56], representada nesta figura pela sigla "CGC", através do estudo da evolução de um objeto hadrônico no formalismo do Condensado de Vidro de Cor.

Como pode ser observado, com exceção das equações DGLAP e das equações GLRMQ, derivada no limite de DLLA, as demais equações de evolução aplicadas ao estudo de sistemas densos fornecem a evolução do sistema na variável $\ln (1 / x)$ (com $Q^{2}$ fixo), onde a densidade partônica cresce com o aumento da energia e povoa todo o espaço de fase mais rapidamente, uma vez que os pártons emitidos no processo de evolução possuem tamanho fixo, conforme adiantado nos parágrafos anteriores.

Por fim, algumas observações sobre as equações de evolução no regime de altas densidades são importantes. Conforme mencionado no início da seção 2.3, nessas condições, as informações contidas nas PDFs usuais não são suficientes para descrever a dinâmica da interção entre projétil e alvo e os dipolos de cor passam a ser os graus de liberdade adequados quando do estudo de espalhamentos a altas energias e grandes densidades partônicas. Tendo isso em vista, as equações BK [44-47] e JIMWLK [48-56] mostram ser equações de evolução não lineares na variável $y=\ln (1 / x)$ para a amplitude de dipolo, $\mathcal{N}(x, r)$ e para diversas combinações de pares de linhas de Wilson ${ }^{12}$, respectivamente e não mais para a densidade de gluons, $x g\left(x, Q^{2}\right)$.

\footnotetext{
${ }^{12}$ A rigor, a equação JIMWLK representa uma hierarquia infinita de equações acopladas para correlações de linhas de Wilson [35-40,48-56].
} 


\section{Capítulo 3}

\section{A influência da saturação de pártons nas seções de choque hadrônicas e fotônicas}

Neste capítulo descrevemos os principais ingredientes dinâmicos do formalismo empregado no estudo da influência da saturação de pártons no comportamento da seção de choque inclusiva total com a energia do centro de massa da reação, $\sqrt{s}$, para colisões $p p, p \bar{p}, \gamma p$ e $\gamma \gamma$ e a seção de choque inelástica de produção para colisões $p A r$.

Como é sabido, a propriedade de confinamento da QCD não permite a observação isolada de quarks e gluons. Portanto, em experiências realizadas no laboratório, apenas hádrons e léptons chegam aos detectores. A tentativa de descrever os processos hadrônicos que ocorrem a altas energias através dos subprocessos partônicos deu origem a uma série de modelos onde estes subprocessos são calculados via pQCD e levam em consideração a formação de jatos de pequenos momentos transversais, chamados de "minijatos". Estes modelos se mostraram promissores e ganharam destaque após descreverem dados experimentais em colisões $p p(\bar{p})$ no final da década de 1980 [207-209].

Desde o início da nova geração de colisores de altas energias, começando com o 
Tevatron no Fermilab, situado nos Estados Unidos da América e o HERA no DESY, situado na Alemanha, no início das décadas de 1980 e 1990, respectivamente, passando pelo RHIC no BNL, também nos Estados Unidos da América, no início dos anos 2000 e atualmente no LHC no CERN, localizado na Suíça, existem ainda muitas proposições teóricas que estão a espera de um respaldo experimental. Entre elas esta o comportamento das seções de choque a altas energias, a universalidade do comportamento das seções de choque hadrônicas a altas energias, a natureza da produção múltipla de partículas e a possibilidade da criação de estados de matéria fortemente interagente, entre outras.

Nesse capítulo vamos nos restringir apenas às questões que dizem respeito às seções de choque, principalmente no que se refere ao comportamento destas a altas energias. Para isto vamos, em um primeiro instante, voltar nossas atenções para a seção de choque hadrônica total mais elementar, a seção de choque associada aos espalhamentos $p p$ e $p \bar{p}$.

Na década de 1980 uma explicação para o crescimento dessas seções de choque com a energia foi proposta por Gaisser e Halzen [210], dando origem a um dos primeiros modelos de minijatos, na qual as interações entre os pártons dos hádrons colidentes eram responsáveis por tal comportamento. Nesse cenário, a altíssimas energias, a mais significativa contribuição para a seção de choque hadrônica são as interações entre gluons de baixos momentos que ditam, nesse regime, um rápido crescimento desta com $\sqrt{s}[209]$.

\subsection{A seção de choque de minijatos}

A altas energias existe um significativo crescimento do número de gluons no interior de hádrons colidentes $\left(h_{1}\right.$ e $\left.h_{2}\right)$, conforme apontado no início do capítulo anterior. Nesse tipo de colisão, em ordem dominante na constante de acoplamento forte da QCD, utiliza-se a pQCD e, nessas condições, os processos que contribuem para a 
seção de choque de minijatos são processos elementares, de dois corpos para dois corpos $(i j \rightarrow k l)$, cujo estado final é composto de dois jatos opostos ("back-to-back") com o mesmo momento transversal. A seção de choque de minijatos é então obtida através da convolução das distribuições de momento $f_{i / h_{1}}$ e $f_{j / h_{2}}$ dos pártons do tipo $i$ e $j$ nos hádrons que colidem, $h_{1}$ e $h_{2}$, com a seção de choque elementar para cada processo partônico que contribui, escrita em termos das variáveis de Mandelstan a nível partônico $(\hat{s}, \hat{t}, \hat{u})$.

Na produção de pártons "semihard", que são jatos com momento transversal maior que um dado momento transversal mínimo $\left(p_{T} \geq p_{T_{m i n}}\right)$, que define também a escala de momento em que a pQCD é válida, a seção de choque para cada tipo de párton provado pode ser escrita como sendo [209,211-213]:

$$
\begin{aligned}
\frac{d \sigma_{k l}}{d y}\left(\sqrt{s}, p_{T_{m i n}}\right) & =K \int d p_{T}^{2} d y_{2} \sum_{i, j,<k, l>} x_{1} f_{i / h_{1}}\left(x_{1}, Q^{2}\right) x_{2} f_{j / h_{2}}\left(x_{2}, Q^{2}\right) \times \\
& \times\left[\delta_{f k} \frac{d \hat{\sigma}^{i j \rightarrow k l}}{d \hat{t}}(\hat{t}, \hat{u})+\delta_{f l} \frac{d \hat{\sigma}^{i j \rightarrow k l}}{d \hat{t}}(\hat{u}, \hat{t})\right] \frac{1}{1+\delta_{k l}},
\end{aligned}
$$

onde $x_{1}$ e $x_{2}$ são as frações de momento (variáveis de Bjorken) carregadas pelos pártons $i$ e $j$ nos hádrons $h_{1}$ e $h_{2}, Q^{2}$ e $d \hat{\sigma} / d \hat{t}$ representam, respectivamente, a escala de momento em que as distribuições de momento $f_{i / h_{1}}$ e $f_{j / h_{2}}$ são provadas e a seção de choque elementar para o processo $i j \rightarrow k l$.

Nessa expressão as rapidez desses pártons, $y \equiv y_{1}$ e $y_{2}$, estão relacionadas aos momentos fracionários acima por $x_{1,2}=\xi\left(e^{ \pm y}+e^{ \pm y_{2}}\right)$, onde $\xi \equiv p_{T} / \sqrt{s}$. Uma vez que $0 \leq x_{i} \leq 1$, a rapidez dos pártons semihard produzidos $(k l)$ fica restrita ao alcance $|y| \leq \ln \left(\beta+\sqrt{\beta^{2}-1}\right)$, onde $\beta=\sqrt{s} /\left(2 p_{T_{\min }}\right)$. O fator $1 /\left(1+\delta_{k l}\right)$ é de cunho estatístico e evita a dupla contagem de eventos quando um evento particular possui partículas idênticas no estado final.

As regiões de integração nas variáveis $p_{T}^{2}$ e $y_{2}$ são dadas por $p_{T_{m i n}}^{2} \leq p_{T}^{2} \leq$ $s /\left[4 \cosh ^{2}(y)\right]$ e $-\ln \left(\xi^{-1}-e^{-y}\right) \leq y_{2} \leq+\ln \left(\xi^{-1}-e^{y}\right)$. Os invariantes $\hat{t}$ e $\hat{u}$ estão relacionados a essas quantidades por $\hat{t}, \hat{u}=-p_{T}^{2}\left[1+e^{\mp\left(y-y_{2}\right)}\right]$. O fator $K(\sim 1 \div 2.5)$ 
na equação (3.1) leva em consideração correções em ordens mais altas na constante de acoplamento forte, presente nas seções de choque elementares apresentadas a seguir, e dada pela equação (1.40).

Como é frequente nesse tipo de cálculo, são assumidos pártons sem massa para os estados inicial e final $(\hat{s}+\hat{t}+\hat{u}=0)$. Em nossos cálculos assumimos o número de sabores ativos para quarks e antiquarks, $n_{f}$, igual a 4 . Para todos os resultados apresentados neste capítulo decidiu-se ainda assumir uma postura conservadora fixando-se $K=1$ de modo a não levar em consideração correções de mais altas ordens em $\alpha_{s}$. A escala $Q^{2}$ que figura nas distribuições de pártons foi fixada no momento transversal dos pártons, isto é, $Q^{2}=p_{T}^{2}$.

A soma sobre os estados iniciais inclui, a princípio, todas as combinações de duas espécies de pártons, $i j=g g, g q, q g, g \bar{q}, \bar{q} g, q q, q \bar{q}, \bar{q} q, \bar{q} \bar{q}$, enquanto que os estados finais consistem na soma de todos os pares $<k l>=g g, g q, g \bar{q}, q q, q \bar{q}, \bar{q} \bar{q}$, onde " $<>$ "indica que não deve ser considerada a troca mútua nos índices $k$ e $l$.

A altas energias, a produção total de minijatos é dominada por interações gluônicas. Por essa razão (e também para tornar os cálculos mais simples), consideramos nesse estudo apenas os processos $g g \rightarrow g g, g q(\bar{q}) \rightarrow g q(\bar{q})$ e $g g \rightarrow q \bar{q}$. As seções de choque elementares $\left(\hat{\sigma}^{i j \rightarrow k l}\right)$ em ordem dominante em $\alpha_{s}$ para esses processos são dadas por $[102,209,214]$ :

$$
\begin{gathered}
\frac{d \hat{\sigma}^{g g \rightarrow g g}}{d \hat{t}}(\hat{t}, \hat{u})=\frac{\pi \alpha_{s}^{2}\left(Q^{2}\right)}{\hat{s}^{2}} \frac{9}{2}\left(3-\frac{\hat{t} \hat{u}}{\hat{s}^{2}}-\frac{\hat{u} \hat{s}}{\hat{t}^{2}}-\frac{\hat{s} \hat{t}}{\hat{u}^{2}}\right), \\
\frac{d \hat{\sigma}^{g q(\bar{q}) \rightarrow g q(\bar{q})}}{d \hat{t}}(\hat{t}, \hat{u})=\frac{\pi \alpha_{s}^{2}\left(Q^{2}\right)}{\hat{s}^{2}}\left(\frac{\hat{s}^{2}+\hat{u}^{2}}{\hat{t}^{2}}-\frac{4}{9} \frac{\hat{s}^{2}+\hat{u}^{2}}{\hat{s} \hat{u}}\right), \\
\frac{d \hat{\sigma}^{g g \rightarrow q \bar{q}}}{d \hat{t}}(\hat{t}, \hat{u})=\frac{\pi \alpha_{s}^{2}\left(Q^{2}\right)}{\hat{s}^{2}} \frac{3}{8}\left(\frac{4}{9} \frac{\hat{t}^{2}+\hat{u}^{2}}{\hat{t} \hat{u}}-\frac{\hat{t}^{2}+\hat{u}^{2}}{\hat{s}^{2}}\right) .
\end{gathered}
$$

Devido à simetria entre os canais $\hat{t}$ e $\hat{u}$ tem-se a igualdade,

$$
\frac{d \hat{\sigma}^{i j \rightarrow k l}(\hat{t}, \hat{u})}{d \hat{t}}=\frac{d \hat{\sigma}^{j i \rightarrow k l}(\hat{u}, \hat{t})}{d \hat{t}}
$$


e após a soma sobre os estados partônicos finais, $\operatorname{com} k l=g g, g q(\bar{q}), q \bar{q}$, a equação $(3.1)$ fornece a seção de choque para a produção de gluons, quarks e antiquarks $\left(d \sigma^{g g} / d y\right.$, $d \sigma^{g q(\bar{q})} / d y, d \sigma^{q \bar{q}} / d y$, respectivamente) $\operatorname{com} p_{T}>p_{T_{\min }}$ em colisões $p p(\bar{p})$. Isso significa que a seção de choque total de produção de minijatos é, nessas condições, a soma das contribuições acima:

$$
\sigma_{\text {tot }, p p}^{m j}(s)=\sigma_{\text {hard }}^{g g}(s)+\sigma_{\text {hard }}^{g q(\bar{q})}(s)+\sigma_{\text {hard }}^{q \bar{q}}(s) .
$$

Nessa expressão, as seções de choque para a produção de um minijato de tipo $k l$ são normalizadas tal que:

$$
\int d y \frac{d \sigma^{k l}}{d y} \equiv 2 \sigma_{\text {hard }}^{k l}(s),
$$

onde o fator 2 leva em consideração que em um evento semihard dois pártons interagem e dois minijatos (back-to-back) são produzidos com $k l=g g, g q(\bar{q}), q \bar{q}$.

Na proposta de Gaisser e Halzen, a idéia básica é que a seção de choque total hadrônica pode ser decomposta como [210]

$$
\sigma_{\text {tot }}^{h_{1} h_{2}}=\sigma_{0}+\sigma_{p Q C D}
$$

onde $\sigma_{0}$ caracteriza uma contribuição não-perturbativa de $\sigma_{\text {tot }}$ (geralmente admitida como independente de $\sqrt{s}[215])$ e $\sigma_{p Q C D}$ caracteriza a contribuição perturbativa, identificada com a seção de choque de produção de minijatos, dada pela equação (3.6).

Modelar $\sigma_{\text {tot }}^{h_{1} h_{2}}$ apenas como uma soma direta de uma contribuição não-perturbativa e outra perturbativa como em (3.8) apresenta, no entanto, sérios problemas que impossibilitam a descrição dos dados experimentais hoje existentes (como veremos posteriormente); a despeito do fato de que os dados experimentais relativos à seção de choque de minijatos $(\sqrt{s}=200-900 \mathrm{GeV})$ foram satisfatoriamente descritos à época com esse modelo [209].

De modo ilustrativo, na Figura 3.1 são apresentados os resultados para a seção de 
choque total $p p(\bar{p})$ segundo a proposta das referências [209,210], dada pela equação (3.8), onde $\sigma_{0}=42 m b$ e $\sigma_{p Q C D}$ é dada por (3.6). Para estes cálculos foram utilizadas as distribuições de pártons CTEQ5L [91] e CTEQ6L [92] e, como único parâmetro livre do modelo, foram feitas as escolhas $p_{T_{m i n}}^{C T E Q 5 L}=2,2 \mathrm{GeV}$ e $p_{T_{m i n}}^{C T E Q 6 L}=2,5 \mathrm{GeV}$. O número de sabores de pártons, assim como os valores de $\Lambda_{Q C D}$, assumidos nesses cálculos são os mesmos adotados nessas distribuições. Os dados experimentais são das referências [216-220].

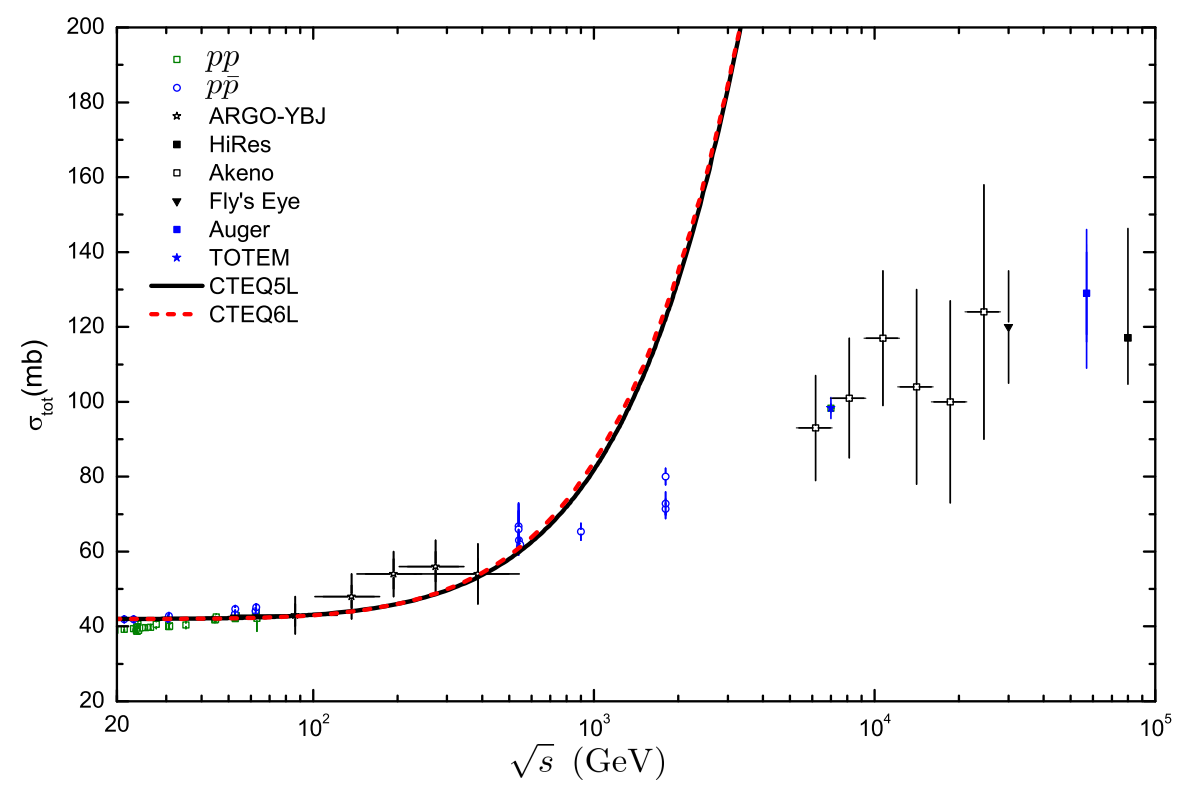

Figura 3.1: Seção de choque total $p p(\bar{p})$ segundo o modelo de minijatos com $\sigma_{0}=42 \mathrm{mb}$. Adaptado de [72].

Como pode ser visto nesta figura (e apontado nas referências [211-213,221-234]) o modelo de minijatos (equação (3.8)) apresenta um crescimento muito forte com $\sqrt{s}$ que viola o limite de Froissart (equação (1.59)) que fornece a taxa máxima de crescimento para as seções de choque totais hadrônicas sem que ocorra a violação da unitaridade da matriz de espalhamento. Isso ocorre devido ao rápido crescimento do número de gluons na região de altas energias.

Como dito anteriormente, o parâmetro $p_{T_{m i n}}$ determina a escala em que os processos semihard passam a ser dominantes. Ainda nos dias de hoje discute-se acerca de seu 
valor ou de uma possível dependência sua com a energia. Em ambos os casos essa liberdade de escolha surge do fato de não haver uma separação bem definida entre as regiões dos processos perturbativos e não-perturbativos.

A seção de choque de minijatos possui, obviamente, uma grande dependência com o valor escolhido para $p_{T_{m i n}}$, cujo papel principal é o de apressar ou retardar a contribuição dos minijatos com a energia. Além disso, quanto maior o seu valor, menor é a contribuição de $\sigma_{p Q C D}$ para $\sigma_{t o t}^{p p(\bar{p})}$, pois deixa-se de computar na equação (3.1) a produção de minijatos com $p_{T} \leq p_{T_{m i n}}$, que é justamente onde se encontra a contribuição dominante. Portanto $\sigma_{\text {tot }}^{p p(\bar{p})}$ apresenta uma taxa de crescimento cada vez menor com $\sqrt{s}$ com o aumento de $p_{T_{\min }}$.

\subsection{Formalismo eiconal}

Um cálculo para a seção de choque hadrônica a altas energias, que seja consistente com a unitaridade da matriz de espalhamento, pode ser realizado através do uso do formalismo eiconal [101,221, 225-230, 235-237], que historicamente foi desenvolvido para contornar esse problema.

Esse formalismo se baseia em uma representação no parâmetro de impacto, $\vec{b}$, e, dentre as diversas opções para a dedução das expressões das seções de choque total, inelástica e elástica, adotamos nesse estudo a abordagem proposta por Glauber [238] por ser mais transparente quando comparado a demais métodos.

\subsubsection{A aproximação eiconal}

O formalismo proposto por Glauber consiste em estudar o limite de altas energias do espalhamento de um partícula por um potencial não-relativístico, $V(\vec{r})$, utilizando 
a equação de Schrödinger

$$
\left(\nabla^{2}-U(\vec{r})+k^{2}\right) \psi(\vec{r})=0
$$

onde

$$
k^{2}=\frac{2 \mu}{\hbar^{2}} E, U(\vec{r})=\frac{2 \mu}{\hbar^{2}} V(\vec{r}) .
$$

Nesta expressão $\mu$ representa a massa reduzida do sistema considerado e $E$ é a energia desse sistema. A chamada aproximação eiconal, válida quando tomamos o limite de altas energias, $E \gg V(\vec{r})$, e pequenos ângulos de espalhamento ${ }^{1}$, se traduz no fato de o comprimento de onda da partícula incidente, $k$ na equação acima, ser muito menor que o alcance da interação, $a: k a \gg 1$ [101,240,241].

Supondo-se que a solução da equação (3.9) possa ser escrita como

$$
\psi(\vec{r})=\varphi(\vec{r}) e^{i \vec{k} \cdot \vec{r}}
$$

substituindo-se (3.11) em (3.9) obtém-se a seguinte equação para $\varphi(\vec{r})$

$$
\left(\nabla^{2}+2 i \vec{k} \cdot \vec{\nabla}-U(\vec{r})\right) \varphi(\vec{r})=0
$$

com a condição de contorno $\varphi(\vec{r})=\varphi(x, y, z=-\infty)=1$.

Utilizando-se o regime de validade da aproximação eiconal, pode-se supor que $V(\vec{r})$ e $\varphi(\vec{r})$ variam muito lentamente se comparados com $e^{i \vec{k} \cdot \vec{r}}$ e é possível descartar o termo proporcional a $\nabla^{2}$ para obter-se

$$
\left(2 i k \frac{\partial}{\partial z}-U(x, y, z)\right) \varphi(x, y, z)=0
$$

onde foi utilizado o fato de que $\vec{k}$ encontra-se orientado na direção $z, \vec{k}=k \hat{u}_{z}$, sendo

\footnotetext{
${ }^{1}$ Embora aqui estejamos considerando tais limites para a dedução dessas expressões, pode-se mostrar que o formalismo no parâmetro de impacto é válido para qualquer valor da energia e do ângulo. Esta demonstração, baseada nas transformações de Watson-Sommerfeld, foi feita por M. M. Islam e pode ser encontrada em [239].
} 
$\hat{u}_{z}$ o versor unitário que define a direção de $z$.

Aplicando-se a condição de contorno mencionada acima à equação (3.13) temos que a função de onda $\psi(\vec{r})$ da partícula espalhada é igual a

$$
\psi(x, y, z)=\varphi(\vec{r}) e^{i k z}=\exp \left[i k z-\frac{i}{2 k} \int_{-\infty}^{z} U\left(x, y, z^{\prime}\right) d z^{\prime}\right]
$$

Como mostra a Figura 3.2, o vetor $\vec{r}$ pode ser decomposto como

$$
\vec{r}=\vec{b}+z \hat{u}_{z}
$$

onde $\vec{b}$ é um vetor perpendicular à direção de propagação, isto é o parâmetro de impacto, o que nos permite reescrever a equação (3.14) como

$$
\psi(\vec{r})=\exp \left[i \vec{k} \cdot \vec{r}-\frac{i}{2 k} \int_{-\infty}^{z} U\left(\vec{b}, z^{\prime}\right) d z^{\prime}\right]
$$

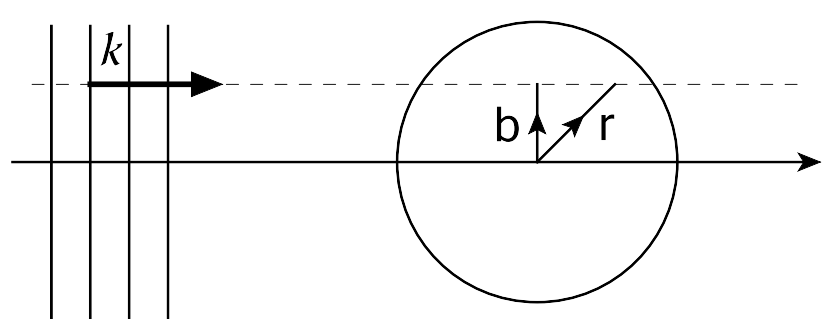

Figura 3.2: Espalhamento de uma onda plana por um potencial. Extraído de [101].

Uma vez obtida a solução completa para a função de onda da partícula espalhada é possível obter a amplitude de espalhamento $f\left(\vec{k}, \overrightarrow{k^{\prime}}\right)$ através da expressão [101,238, $240,241]$

$$
f\left(\vec{k}, \overrightarrow{k^{\prime}}\right)=-\frac{1}{4 \pi} \int e^{-i \overrightarrow{k^{\prime}} \cdot \vec{r}} U\left(\overrightarrow{r^{\prime}}\right) \psi\left(\overrightarrow{r^{\prime}}\right) d^{3} \overrightarrow{r^{\prime}}
$$

Substituindo-se (3.16) em (3.17), e notando-se que $d^{3} \overrightarrow{r^{\prime}}=d^{2} \vec{b} d z$ temos

$$
f\left(\vec{k}, \overrightarrow{k^{\prime}}\right)=-\frac{1}{4 \pi} \int_{-\infty}^{\infty} d z \int d^{2} \vec{b} e^{-i \vec{q} \cdot\left(\vec{b}+k \hat{u}_{z}\right)} U(\vec{b}, z) e^{-\frac{i}{2 k} \int_{-\infty}^{z} U\left(\vec{b}, z^{\prime}\right) d z^{\prime}}
$$


onde $\vec{q}=\overrightarrow{k^{\prime}}-\vec{k}$.

Para pequenos ângulos de espalhamento o vetor $\vec{q}$ é quase ortogonal a $\vec{k}$ e, então, pode-se escrever $\vec{q} \cdot \vec{r} \approx \vec{q} \cdot \vec{b}$. Utilizando-se a identidade

$$
U(\vec{b}, z) e^{-\frac{i}{2 k} \int_{-\infty}^{z} U\left(\vec{b}, z^{\prime}\right) d z^{\prime}}=2 i k \frac{\partial}{\partial z} e^{-\frac{i}{2 k} \int_{-\infty}^{z} U\left(\vec{b}, z^{\prime}\right) d z^{\prime}}
$$

a integração em $d z$ na equação (3.18) é facilmente realizada conduzindo à expressão final da amplitude de espalhamento

$$
f\left(\vec{k}, \overrightarrow{k^{\prime}}\right)=f(\vec{q})=\frac{i k}{2 \pi} \int d^{2} \vec{b} e^{-i \vec{q} \cdot \vec{b}}\left(1-e^{i \chi(\vec{b})}\right)
$$

onde a chamada "função eiconal", $\chi(\vec{b})$, é definida como

$$
\chi(\vec{b})=-\frac{i}{2 k} \int_{-\infty}^{\infty} U(\vec{b}, z) d z
$$

A quantidade

$$
\Gamma(\vec{b}) \equiv 1-e^{i \chi(\vec{b})}
$$

é comumente chamada de "função de perfil" em analogia com a óptica e pode ser obtida a partir de $f\left(\vec{k}, \overrightarrow{k^{\prime}}\right)$ através da transformada de Fourier da equação (3.20),

$$
\Gamma(\vec{b})=-\frac{2 \pi i}{k} \int d^{2} \vec{q} f(\vec{q}) e^{i \vec{q} \cdot \vec{b}}
$$

Uma vez conhecida a expressão para a amplitude de espalhamento é possível obter a expressão para as seções de choque total, elástica e inelástica ${ }^{2}$

$$
\begin{array}{rlr}
\sigma_{\text {tot }}(s) & =\left.\frac{4 \pi}{k} \operatorname{Imf}(\vec{q})\right|_{\vec{q}=0}, \quad \text { (Teorema Óptico) } \\
\sigma_{\mathrm{el}}(s) & \equiv \int|f(\vec{q})|^{2} d \Omega=\frac{1}{k^{2}} \int|f(\vec{q})|^{2} d^{2} \vec{q} \\
\sigma_{\text {inel }}(s) & \equiv \sigma_{\text {tot }}(s)-\sigma_{\mathrm{el}}(s)
\end{array}
$$

\footnotetext{
${ }^{2}$ A rigor o Teorema Óptico é calculado com o ânglo de espalhamento, $\theta$, nulo. Usando-se $|\vec{q}|=$ $k \sin (\theta)$ temos que $\vec{q}=0$ corresponde ao caso $\theta=0$.
} 
onde foi utilizado $d^{2} \vec{q}=\pi d q^{2} \simeq k^{2}(2 \pi \theta d \theta) \simeq k^{2} d \Omega$ na equação (3.25).

Uma grande vantagem do formalismo eiconal é a garantia imediata da unitaridade da matriz de espalhamento. Esta garantia vem através da utilização do Teorema Óptico que, na Mecânica Quântica, é uma consequência da conservação da corrente de probabilidade.

Por fim, utilizando-se as equações (3.24)-(3.26) tem-se as seguintes expressões para as seções de choque total, elástica e inelástica na aproximação eiconal:

$$
\begin{aligned}
\sigma_{\text {tot }}(s) & =2 \int\left[1-e^{-\chi_{I}(b, s)} \cos \left(\chi_{R}(b, s)\right)\right] d^{2} \vec{b}, \\
\sigma_{\text {el }}(s) & =\int\left|1-e^{-\chi_{I}(b, s)+i \chi_{R}(b, s)}\right|^{2} d^{2} \vec{b}, \\
\sigma_{\text {inel }}(s) & =\int\left[1-e^{-2 \chi_{I}(b, s)}\right] d^{2} \vec{b} .
\end{aligned}
$$

Nesse formalismo, a função eiconal $\chi(b, s)$ nas expressões acima contém a dependência na distribuição de matéria e a dinâmica envolvida nas colisões das partículas colidentes com a energia e o parâmetro de impacto, e é dada por

$$
\chi(b, s)=\chi_{R}(b, s)+i \chi_{I}(b, s) .
$$

O modo como se constrói as partes real, $\chi_{R}(b, s)$, e imaginária, $\chi_{I}(b, s)$, da função eiconal é o que diferencia cada um dos modelos que dela se utilizam. A seguir mostrase que é possível estender o formalismo eiconal para colisões próton-núcleo, no entanto, antes disso, é importante mencionar que é possível deduzir o limite de Froissart (equação (1.59)) a partir do formalismo eiconal. Esta dedução, bem menos geral que a dedução original pode ser encontrada na referência [242]. 


\subsection{Colisões hádron-núcleo no formalismo eiconal: a aproximação de Glauber}

As expressões (3.27)-(3.29) são válidas para o cálculo da seção de choque hadrônica $p p(\bar{p})$. Se considerarmos o espalhamento de um hádron $h$ por um núcleo contendo $A$ nucleons, quando uma partícula colide com um sistema composto podem existir uma série de múltiplos espalhamentos com os $A$ nucleons do alvo. O tratamento geral para uma colisão como esta é muito complicado e, portanto, é costumeiro recorrer-se a algumas aproximações.

Para o caso de um espalhamento hádron-núcleo, $h A$, por exemplo, pode-se generalizar a amplitude de espalhamento do espalhamento hádron-hádron (equação (3.20)) obtendo-se [243]

$$
F_{h A}(\vec{q})=\frac{i k}{2 \pi} \int d^{2} \vec{b} e^{-i \vec{q} \cdot \vec{b}}\left\langle A^{\prime}\left|\left(1-e^{i \chi_{A}\left(\vec{b}, \vec{b}_{1}, \vec{b}_{2}, \ldots, \vec{b}_{A}\right)}\right)\right| A\right\rangle,
$$

onde $\vec{b}, \vec{b}_{1}, \vec{b}_{2}, \ldots, \vec{b}_{A}$ são as posições dos nucleons relativas ao eixo da colisão e $|A\rangle$ e $\left|A^{\prime}\right\rangle$ representam os estados inicial e final do núcleo alvo, que estão normalizados segundo a condição:

$$
\int d^{3} \vec{r}_{1} d^{3} \vec{r}_{2} \ldots d^{3} \vec{r}_{A} \delta^{(3)}\left(\vec{r}_{1}+\ldots+\vec{r}_{A}\right)\left|\varphi_{A}\left(\vec{r}_{1}, \vec{r}_{2}, \ldots, \vec{r}_{A}\right)\right|^{2}=1
$$

Supondo-se que cada interação que ocorrer com o hádron projétil seja tratada como uma interação incoerente hádron-nucleon, a função eiconal resultante será a soma de cada função eiconal decorrente de todas as interações hádron-nucleon,

$$
\chi_{A}\left(\vec{b}, \vec{b}_{1}, \vec{b}_{2}, \ldots, \vec{b}_{A}\right)=\sum_{i=1}^{A} \chi\left(\vec{b}-\overrightarrow{b_{i}}\right) .
$$

A aproximação que acabamos de considerar é conhecida como "aproximação de 
Glauber" $[192,193]$ e implica que o projétil pode interagir com cada nucleon do alvo apenas uma única vez, de modo que o número máximo de interações é igual a quantidade de nucleons do núcleo alvo, $A$. Uma visão geométrica do espalhamento $h A$ é apresentada na Figura 3.3.

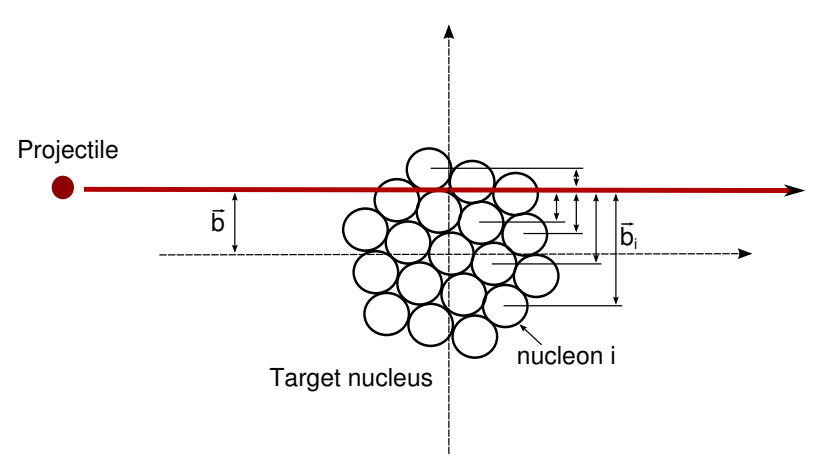

Figura 3.3: Um hádron projétil atingindo um núcleo alvo com um parâmetro de impacto $\vec{b}$. O parâmetro de impacto relativo para cada nucleon, $\vec{b}_{i}$, governa o espalhamento hádron-nucleon que ocorre com cada nucleon $i$. Extraído de [244].

No caso de um espalhamento $h A$ a função de perfil,

$$
\Gamma\left(\vec{b}, \vec{b}_{1}, \vec{b}_{2}, \ldots \vec{b}_{A}\right)=1-e^{i \chi_{A}\left(\vec{b}, \vec{b}_{1}, \vec{b}_{2}, \ldots, \vec{b}_{A}\right)}
$$

possui uma interpretação muito interessante em termos dos espalhamentos múltiplos que podem ocorrer entre o hádron e os nucleons do núcleo.

Utilizando-se a aproximação de Glauber, assim como a equação (2.20), a função de perfil $\Gamma\left(\vec{b}, \vec{b}_{1}, \vec{b}_{2}, \ldots \vec{b}_{A}\right)$ "ressoma" múltiplos espalhamentos:

$$
\begin{aligned}
\Gamma\left(\vec{b}, \vec{b}_{1}, \vec{b}_{2}, \ldots \vec{b}_{A}\right) & =1-\prod_{i=1}^{A} e^{i \chi\left(\vec{b}-\vec{b}_{i}\right)}=1-\prod_{i=1}^{A}\left[1-\Gamma\left(\vec{b}-\vec{b}_{i}\right)\right] \\
& =\sum_{i=1}^{A} \Gamma_{i}\left(\vec{b}-\vec{b}_{i}\right)-\sum_{i>j} \Gamma_{i}\left(\vec{b}-\vec{b}_{i}\right) \Gamma_{j}\left(\vec{b}-\vec{b}_{j}\right)+ \\
& +\sum_{i>j>l} \Gamma_{i}\left(\vec{b}-\vec{b}_{i}\right) \Gamma_{j}\left(\vec{b}-\vec{b}_{j}\right) \Gamma_{l}\left(\vec{b}-\vec{b}_{l}\right)-\ldots+ \\
& +(-1)^{A-1} \prod_{i} \Gamma_{i}\left(\vec{b}-\vec{b}_{i}\right)
\end{aligned}
$$


onde o primeiro termo descreve um único espalhamento do projétil com os nucleons do núcleo, o segundo termo descreve um espalhamento duplo do hádron com os nucleons, e assim por diante, sendo que o último termo descreve a situação em que o projétil é espalhado por todos os nucleons do núcleo.

Considerando-se que o hádron seja espalhado sob um pequeno ângulo pelo núcleo, o número de interações $(n)$ do projétil com os nucleons será pequeno quando comparado ao número de nucleons do alvo, $n \ll A$. Como mostra a Figura 3.3, as colisões ocorrem com nucleons que possuem o mesmo valor do parâmetro de impacto do hádron. Assim, para uma distribuição homogênea de nucleons dentro do núcleo, o número de nucleons (e portanto o número de colisões) que satisfazem essa condição é $n \leq A^{1 / 3} \ll A$.

Nessa situação, a fatorização

$$
\int d^{3} \vec{r}_{1} d^{3} \vec{r}_{2} \ldots d^{3} \vec{r}_{A} \delta^{(3)}\left(\vec{r}_{1}+\ldots+\vec{r}_{A}\right)\left|\varphi_{A}\left(\vec{r}_{1}, \vec{r}_{2}, \ldots, \vec{r}_{A}\right)\right|^{2} \rightarrow \rho\left(\vec{r}_{1}\right) \ldots \rho\left(\vec{r}_{n}\right)
$$

onde $\rho\left(\overrightarrow{r_{i}}\right)(i=1, . ., n)$ é a distribuição de densidade do nucleon $i$, é uma boa aproximação para a distribuição da densidade dos $n$ nucleons dentro do núcleo.

A substituição feita na equação (3.36) significa que esses nucleons são considerados partículas independentes que se movem no interior do núcleo. Quando $n \sim A$ a fatorização empregada deixa de ser válida, pois, neste caso, as correlações entre as distribuições dos nucleons passam a ser importantes.

É importante mencionarmos ainda que na equação (3.35) existem $A$ termos caso ocorra uma interação única $(n=1)$ e $A(A-1) / 2$ termos no caso de interações duplas. Para o caso em que ocorrerem $n$ interações, o número de termos é igual a combinação dos $A$ nucleons, $n$ a $n, C_{A}^{n}=A ! /[n !(A-n) !] \approx A^{n} / n$ !, com $n \ll A$. Desta forma, com auxílio das equações (3.35) e (3.36) pode-se realizar a seguinte substituição na equação (3.31):

$$
\int d^{3} \vec{r}_{1} d^{3} \vec{r}_{2} \ldots d^{3} \vec{r}_{A} \delta^{(3)}\left(\vec{r}_{1}+\ldots+\vec{r}_{A}\right)\left|\varphi_{A}\left(\vec{r}_{1}, \vec{r}_{2}, \ldots, \vec{r}_{A}\right)\right|^{2} \Gamma\left(\vec{b}, \vec{b}_{1}, \vec{b}_{2}, \ldots \vec{b}_{A}\right)
$$




$$
\begin{aligned}
& \longrightarrow \sum_{n=1}^{n_{\max }}(-1)^{n+1} \int d^{3} \vec{r}_{1} \ldots d^{3} \vec{r}_{A} \rho\left(\vec{r}_{1}\right) \ldots \rho\left(\vec{r}_{n}\right) \frac{A^{n}}{n !} \Gamma\left(\vec{b}-\vec{b}_{1}\right) \ldots \Gamma\left(\vec{b}-\vec{b}_{n}\right) \\
& =\sum_{n=1}^{n_{\max }} \frac{-1}{n !}\left(\frac{-A}{2 \pi i k} \int d^{2} \vec{q} \int d^{3} \vec{r} e^{-i \vec{q} \cdot\left(\vec{b}-\vec{b}_{n}\right)} f(\vec{q}) \cdot \rho(\vec{r})\right)^{n},
\end{aligned}
$$

onde, na última igualdade, utilizou-se a equação (3.23) para escrever $\Gamma\left(\vec{b}-\vec{b}_{n}\right)$ em termos da amplitude do espalhamento hádron-hádron, $f(\vec{q})$.

Se a amplitude $f(\vec{q})$ possuir uma dependência fraca com $\vec{q}$, o que evidencia o fato do hádron ser um sistema muito pequeno quando comparado a núcleos com $A>10$, pode-se escrever (vide equação (3.24)):

$$
f(\vec{q}) \simeq \frac{i k}{4 \pi} \sigma_{t o t}^{h N}
$$

onde $\sigma_{\text {tot }}^{h N}$ é a seção de choque total da interação hádron-nucleon.

Utilizando-se as equações (3.31), (3.37) e (3.38) a expressão para a amplitude de espalhamento $h A, F_{h A}(\vec{q})$, pode ser obtida facilmente:

$$
F_{h A}(\vec{q})=\frac{i k}{2 \pi} \int d^{2} \vec{b} e^{-i \vec{q} \cdot \vec{b}}\left[1-\sum_{n=0}^{n_{\max }} \frac{1}{n !}\left(-\frac{1}{2} \sigma_{t o t}^{h N} T(\vec{b})\right)^{n}\right]
$$

onde introduz-se a função de perfil nuclear

$$
T(\vec{b})=A \int d z \rho(\vec{b}, z)
$$

que fornece o número de nucleons em um núcleo $A$ por unidade de área ao longo da direção $z$ (usualmente assumida como a direção de propagação feixe incidente), separado do centro do núcleo por um parâmetro de impacto $\vec{b}$ e onde a quantidade $\rho(\vec{b}, z)$ define a densidade de nucleons no núcleo em função desses parâmetros.

Por fim, considerando-se o caso em que $n_{\max } \sim A^{1 / 3}$ e assumindo-se que $A$ é um número muito grande, pode-se tomar o limite em que $n_{\max } \rightarrow \infty$. Neste caso a série em $n$ na equação (3.39) pode ser identificada como a série de uma função exponencial e, 
como resultado final para a amplitude de espalhamento elástica hádron-núcleo, tem-se

$$
F_{h A}(\vec{q})=\frac{i k}{2 \pi} \int d^{2} \vec{b} e^{-i \vec{q} \cdot \vec{b}}\left[1-e^{-\frac{1}{2} \sigma_{t o t}^{h N} T(\vec{b})}\right]
$$

Nessas condições, as seções de choque total, inelástica e elástica em colisões $h A$ podem ser escritas como sendo dadas por

$$
\begin{aligned}
\sigma_{\text {tot }}^{h A}\left(E_{l a b}\right) & =2 \int d^{2} \vec{b}\left[1-e^{-\frac{1}{2} \sigma_{t o t}^{h N} T(\vec{b})}\right], \\
\sigma_{\mathrm{el}}^{h A}\left(E_{l a b}\right) & =\int d^{2} \vec{b}\left|1-e^{-\frac{1}{2} \sigma_{t o t}^{h N} T(\vec{b})}\right|^{2}, \\
\sigma_{\text {inel }}^{h A}\left(E_{l a b}\right) & =\int d^{2} \vec{b}\left[1-e^{-\sigma_{\text {tot }}^{h N} T(\vec{b})}\right],
\end{aligned}
$$

onde $E_{l a b}$ é a energia da colisão no referêncial do laboratório, relacionada à energia do centro de massa de uma colisão hádron-núcleo, $\sqrt{s}$, por intermédio da expressão:

$$
E_{l a b}=\frac{s-m_{1}^{2}-m_{2}^{2}}{2 m_{2}}
$$

\subsection{Modelo de minijatos eiconalizado com saturação de pártons}

Como apontado anteriormente, quando se utiliza o formalismo eiconal, o modo como se constrói a função eiconal, $\chi(b, s)$ (equação (3.30)), é o que diferencia os diversos modelos existentes. Algumas hipóteses, porém, são comuns à grande maioria destes modelos e é usual assumir a validade da fatorização

$$
\chi(b, s)=W(b) A(s)
$$

onde $W(b)$ é normalizada tal que $\int W(b) d^{2} \vec{b}=1$.

Essa função é conhecida como função de "overlap"e fornece a informação sobre 
a parte espacial da colisão, enquanto que $A(s)$ é (comumente) apenas uma função dependente da energia da colisão.

Os modelos atuais, baseados no formalismo do parâmetro de impacto [245-251], são todos frutos de adaptações e/ou sofisticações dos primeiros modelos eiconais existentes [225-230] e, quando comparados, a maior diferença entre eles está no ingrediente que contém toda a informação dinâmica da colisão, a função $A(s)$.

Visando a construção de um modelo eiconal que considera os efeitos da saturação de pártons e possa ser utilizado para descrever os dados experimentais das seções de choque totais $p p$ e $p \bar{p}$ e da seção de choque inelástica de produção $p-A r$, simultâneamente, utilizamo-nos nessa tese dos trabalhos das referências [221, 225-230] como guia, e assumimos que as seções de choque total, elástica e inelástica para uma colisão hádron-hádron são dadas pelas expressões (3.27)-(3.29). Para o caso de colisões hádron-núcleo as mesmas quantidades são dadas pelas equações (3.42)-(3.44).

Seguindo a idéia dos modelos inspirados em QCD, assumimos igualmente que o crescimento da seção de choque hadrônica é devido ao aumento da produção de minijatos com o aumento da energia do centro de massa da colisão, $\sqrt{s}$ e utilizamos a equação (3.30), inspirados no modelo da referência [210], para escrever a parte imaginária da função eiconal como sendo

$$
\chi_{I}(b, s)=\chi_{s o f t}(b, s)+\chi_{p Q C D}(b, s),
$$

onde a primeira parcela diz respeito às contribuições não perturbativas e a segunda contém as contribuições perturbativas do processo.

A parte real, $\chi_{R}(s, b)$, deixa de ser importante a altas energias [248-251] e foi tomada como nula nesse estudo. O fato de considerarmos $\chi_{R}(s, b) \equiv 0$ na equação (3.30) é baseado no resultado fenomenológico que diz que a razão entre as partes real e imaginária da amplitude de espalhamento elástica frontal é muito pequena a altas energias.

Embora os modelos eiconais baseados na produção de minijatos tenham sido utili- 
zados para descrever a seção de choque total hadrônica, os parâmetros que permitem reproduzir corretamente a dependência com a energia da seção de choque total fornecem uma seção de choque elástica (inelástica), em geral, muito alta (baixa) em relação aos dados experimentais. O mesmo acontece com o nosso modelo eiconal.

Segundo [252], uma possível explicação para a incapacidade de se descrever simultaneamente dois conjuntos de dados experimentais (seções de choque total e inelástica, por exemplo) vem da não inclusão da contribuição dos processos difrativos no formalismo eiconal e que, portanto, deveriam ser tratados a parte. A inclusão dos processos difrativos no formalismo eiconal não é trivial e esta além do escopo desta tese; uma tentativa de inclusão dos processos difrativos no formalismo eiconal pode ser encontrada na referência [252].

Seguindo as referências [211-213,221,225-230], utilizamo-nos aqui do modelo de "fator de forma" onde a quantidade $W(b)$ abaixo é dada pela transformada bidimensional de Fourier do produto de dois fatores de forma,

$$
W(b)=\frac{1}{(2 \pi)^{2}} \int d^{2} \vec{q} e^{i b \cdot q} \mathcal{F}_{1}(q) \mathcal{F}_{2}(q)
$$

Para o caso de colisões $p p(\bar{p})$, os fatores de forma $\mathcal{F}_{1,2}(q)$ foram escolhidos como fatores do tipo dipolo,

$$
\mathcal{F}_{1,2}(q)=\left(\frac{\mu^{2}}{q^{2}+\mu^{2}}\right)^{2}
$$

de forma que

$$
W(b, \mu)=\frac{\mu^{2}}{96 \pi}(\mu b)^{3} K_{3}(\mu b)
$$

onde $\mu$ é um parâmetro de ajuste do modelo e $K_{3}(\mu, b)$ é a função de Bessel modificada de segunda ordem.

No formalismo eiconal existe a possibilidade de se diferenciar as seções de choque $p p$ e $p \bar{p}$ através da modelagem da parte "soft" presente na componente imaginária da função eiconal. Para esta componente, que é importante apenas a baixas energias, 


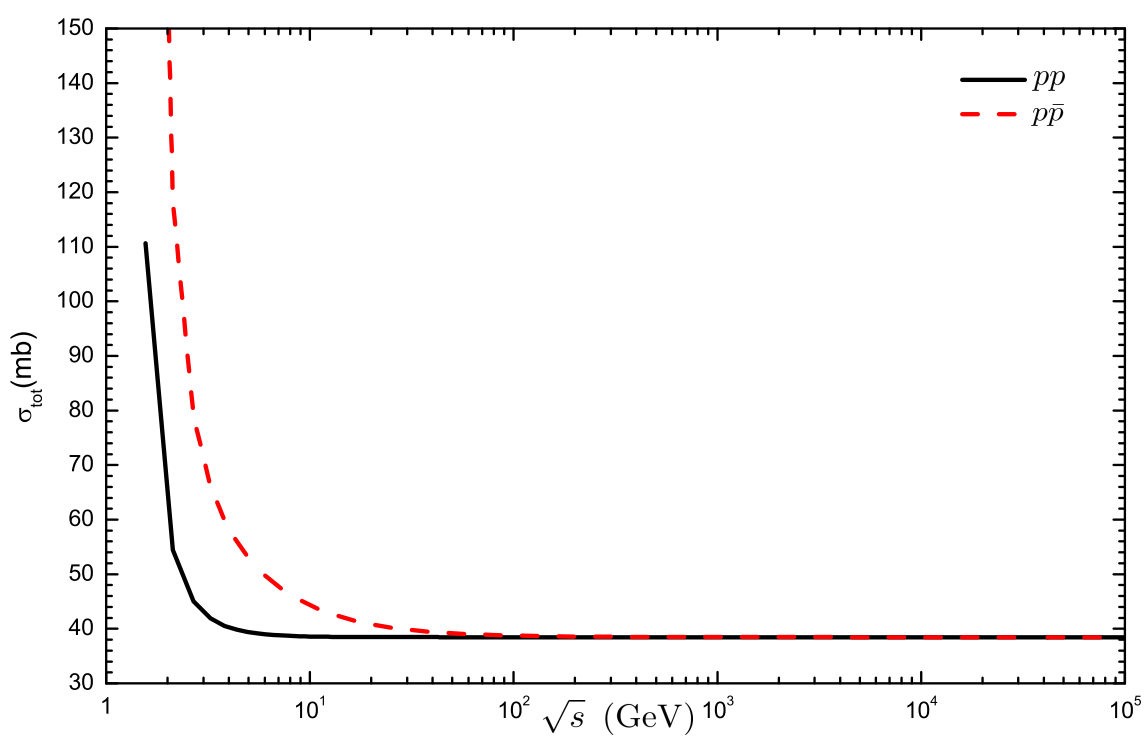

Figura 3.4: Contribuição da parte não perturbativa da função eiconal em colisões $p p(\bar{p})$.

assumimos as mesmas parametrizações da referência [253] nesse estudo:

$$
\begin{aligned}
& A_{p p}\left(E_{l a b}\right)=\sigma_{\text {soft }}^{p p}=47+\frac{46}{E_{l a b}^{1,39}}, \\
& A_{p \bar{p}}\left(E_{l a b}\right)=\sigma_{\text {soft }}^{p \bar{p}}=47+\frac{129}{E_{l a b}^{0,661}}+\frac{357}{E_{l a b}^{2,7}},
\end{aligned}
$$

com $W(b, \mu)=W\left(b, \mu_{\text {soft }}\right)$, por fornecerem um bom ajuste dos dados experimentais na região de baixas energias.

Na Figura 3.4 são apresentados os resultados de nossos cálculos apenas para a contribuição não perturbativa (portanto $\chi_{p Q C D}=0$ ) em colisões $p p$ e $p \bar{p}$ onde o parâmetro $\mu_{\text {soft }}^{2}$ foi fixado em $0,71 \mathrm{GeV}^{2}$. Como pode ser observado, essa contribuição torna-se desprezível a altas energias.

Para a componente perturbativa, $\chi_{p Q C D}(s, b)$, assumimos apenas as contribuições 
partônicas $g g \rightarrow g g, g g \rightarrow g q$ e $g g \rightarrow q \bar{q}$ :

$$
\begin{aligned}
\chi_{\mathrm{pQCD}}(s, b) & =\chi_{\mathrm{gg}}(s, b)+\chi_{\mathrm{gq}}(s, b)+\chi_{\mathrm{q} \overline{\mathrm{q}}}(s, b) \\
& =W\left(b, \mu_{\mathrm{gg}}\right) \sigma_{\mathrm{gg}}(s)+W\left(b, \sqrt{\mu_{\mathrm{q} \overline{\mathrm{q}}} \mu_{\mathrm{gg}}}\right) \sigma_{\mathrm{gq}}(s) \\
& +W\left(b, \mu_{\mathrm{q} \overline{\mathrm{q}}}\right) \sigma_{\mathrm{q} \overline{\mathrm{q}}}(s),
\end{aligned}
$$

onde $\sigma_{k l}(s)$ são as seções de produção de minijatos do tipo gluon-gluon $(g g)$, quarkgluon $(g q)$ e quark-antiquark $(q \bar{q})$, que são dadas pela equação (3.7).

Na equação acima, $\mu_{g g}$ e $\mu_{q \bar{q}}$ são os parâmetros livres de nosso modelo que foram escolhidos de forma a tornar possível a descrição dos dados experimentais. Para $\mu_{g q}$ assumiu-se o vínculo $\mu_{g q}=\sqrt{\mu_{g g} \mu_{q \bar{q}}}$, comumente utilizado em outros trabalhos.

Nesse nosso estudo o principal ingrediente de nosso modelo eiconal é a introdução de termos não lineares na equação de evolução para as distribuições de pártons. No contexto dos modelos de saturação de pártons, adotamos e fizemos uso das distribuições de pártons EHKQS [70,71], cuja evolução é governada pelas equações GLRMQ (equação (2.31)). Como será mostrado a seguir, isto possibilita testar a dinâmica responsável pelo crescimento das seções de choque $p p(\bar{p})$ na presença dos efeitos da saturação de pártons.

Visando comparar os dois regimes da QCD, não linear e linear, onde os efeitos da saturação estão ou não presentes, consideramos neste trabalho as distribuições CTEQ6L [92] e GRV98 [104] como referências. É importante notar que ambas distribuições são governadas pelas equações DGLAP, onde os efeitos da saturação não estão presentes.

Além disso, a estratégia adotada em nossos cálculos foi a seguinte: enquanto que a contribuição não perturbativa do modelo foi parametrizada para descrever as seções de choque $p p$ e $p \bar{p}$ a baixas energias, a altas energias os parâmetros presentes na contribuição perturbativa foram fixados (para cada distribuição de pártons considerada) de modo a descrever os dados mais recentes do LHC $[217,218]$ e o resultado experimental (convertido para colisões pp) das colaborações HiRes [219] e Pierre Auger [220]. 
Uma vez fixados tais parâmetros a dependência energética das seções de choque total e inelástica $p p$ e $p \bar{p}$ fica plenamente estabelecida, possibilitando testar a validade deste modelo em outros processos de interesse, conforme discutido mais adiante.

A seguir apresentamos os resultados obtidos para as seções de choque estudadas.

\subsubsection{Resultados para as seções de choque hadrônicas}

Como dito anteriormente, os parâmetros livres do modelo eiconal que utilizamos haveriam de ser fixados de modo a permitir a descrição das seções de choque $p p$ e $p \bar{p}$ com a energia da colisão. Em todas as figuras apresentadas a seguir as linhas sólidas representam os resultados no regime de evolução não linear (distribuição EHKQS), onde os parâmetros da componente perturbativa foram fixados em $p_{T_{\min }}^{2}=1.51 \mathrm{GeV}^{2}$, $\mu_{g g}=2.00 \mathrm{GeV}, \mu_{q \bar{q}}=0.70 \mathrm{GeV}$. As linhas tracejadas e pontilhadas mostram os resultados no regime de evolução linear do modelo, onde os parâmetros foram fixados, respectivamente, em $p_{T_{\text {min }}}^{2}=2.10 \mathrm{GeV}^{2}, \mu_{g g}=2.03 \mathrm{GeV}, \mu_{q \bar{q}}=0.73 \mathrm{GeV}$ para distribuição CTEQ6L e $p_{T_{\text {min }}}^{2}=1.32 \mathrm{GeV}^{2}, \mu_{g g}=1.88 \mathrm{GeV}, \mu_{q \bar{q}}=1.00 \mathrm{GeV}$ para distribuição GRV98.

A Figura 3.5 mostra as seções de choque totais $p p$ e $p \bar{p}$, dadas pela equação (3.27), utilizando-se os parâmetros acima. Os dados experimentais são das referências [216220]. Como pode ser visto, os resultados obtidos com a equação de evolução não linear também produzem uma boa descrição dos dados experimentais (tão boa quanto os resultados apresentados em [248-251,254,255]) em particular dos dados mais recentes a altas energias (LHC, HiRes e Pierre Auger).

Comparando-se a curva sólida com as curvas tracejada e pontilhada, pode-se concluir que o crescimento da seção de choque a altas energias continua sendo ditado pelo crescimento da distribuição de gluons em pequenos valores de $x$. A evolução não linear (EHKQS), no entanto, conduz a um crescimento um pouco mais moderado da seção de choque com a energia devido aos processos de recombinação de gluons $(g g \rightarrow g)$. 


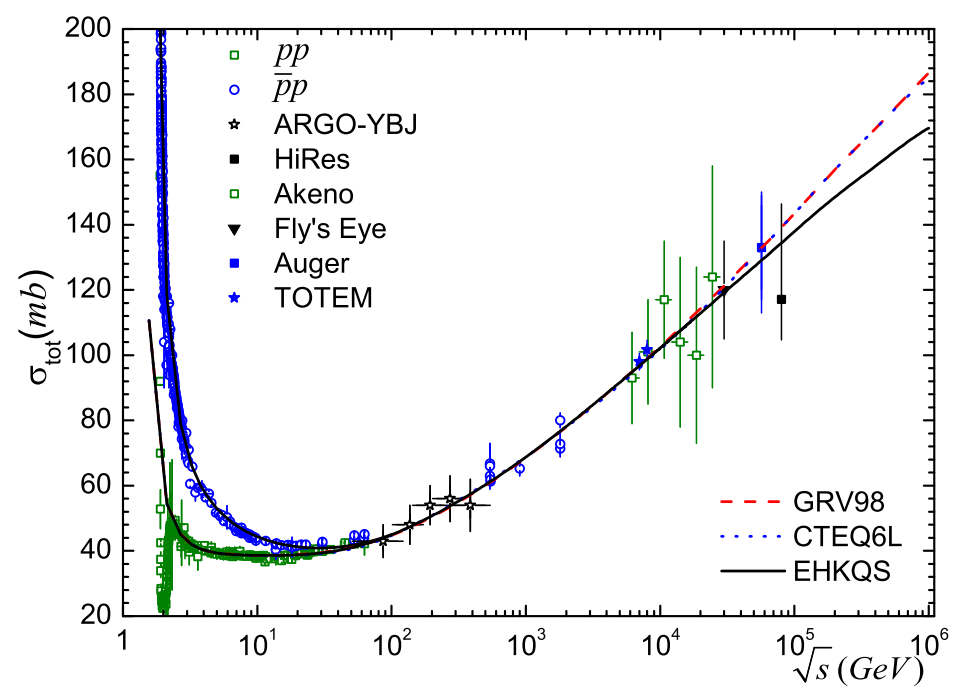

Figura 3.5: Seção de choque total $p p$ e $\bar{p} p$. As linhas sólida, tracejada e pontilhada mostram o resultado do modelo eiconal (equação (3.27)) nos regimes não linear e linear. Os dados experimentais são das referências [216-220]

As Figuras 3.6 (a) e 3.6 (b) mostram, respectivamente, a razão entre as seções de choque da Figura 3.5 e as parametrizações do Particle Data Group (PDG) [216] e Block-Halzen (BH) [224, 256]:

$$
\begin{aligned}
\sigma_{P D G}^{\mp}(s) & =a_{0}+a_{1} A_{1}^{b_{1}} \mp a_{2} A_{1}^{b_{2}}+a_{3} \ln ^{b_{3}}\left(A_{2}\right), \\
\sigma_{B H}^{ \pm}(\nu) & =c_{0}+c_{1} C^{d_{1}} \pm c_{2} C^{d_{2}}+c_{3} \ln (C)+c_{4} \ln ^{d_{3}}(C) .
\end{aligned}
$$

Nessas expressões, o sinal superior (inferior) diz respeito a um espalhamento $p p$ $(p \bar{p}), A_{1} \equiv s / s_{l}, A_{2} \equiv s / s_{h}, C \equiv \nu / m \approx s / 2 m^{2}$, onde $\nu$ e $m$ representam a energia no sistema do laboratório do próton (antipróton) incidente e a massa do próton, respectivamente. Os valores adotados para os parâmetros para cada uma dessas parametrizações são apresentados na tabela 3.1.

Pode-se notar nessas figuras que acima de $\sim 10 \mathrm{TeV}$ todas as curvas caem rapidamente: em $10^{3} \mathrm{TeV}$ as razões com a parametrização PDG são $\sim 0.86$ para os cálculos com CTEQ6L ou GRV98 e 0.79 para EHKQS, e as razões com a parametrização BH são 0.91 para cálculos com CTEQ6L ou GRV98 e 0.84 para EHKQS. 

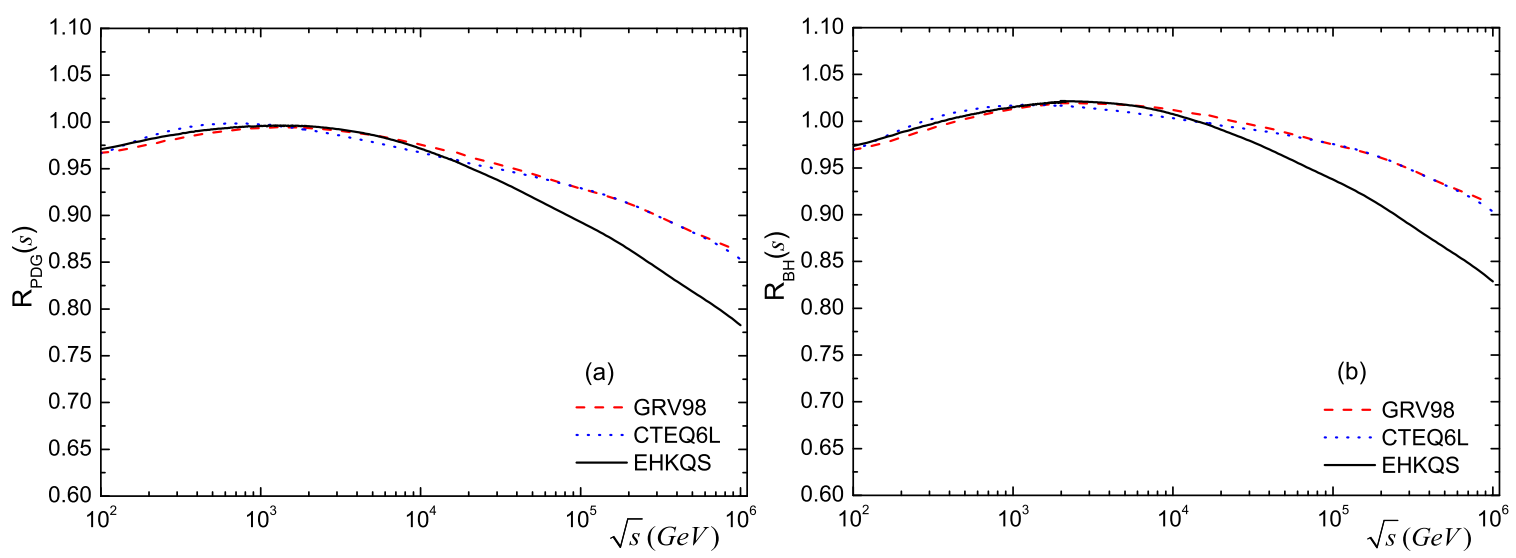

Figura 3.6: Razões entre as seções de choque total apresentadas na Figura 3.5 e as parametrizações dadas pelas equações (3.54) e (3.55).

\begin{tabular}{|c|c|c|c|}
\hline $\mathrm{PDG}$ & {$[216]$} & $\mathrm{BH}$ & {$[224,256]$} \\
\hline$a_{0}(\mathrm{mb})$ & $35.35 \pm 0.48$ & $c_{0}(\mathrm{mb})$ & 37.32 \\
\hline$a_{1}(\mathrm{mb})$ & $42.53 \pm 1.35$ & $c_{1}(\mathrm{mb})$ & 37.10 \\
\hline$a_{2}(\mathrm{mb})$ & $33.34 \pm 1.04$ & $c_{2}(\mathrm{mb})$ & -28.56 \\
\hline$a_{3}(\mathrm{mb})$ & $0.308 \pm 0.010$ & $c_{3}(\mathrm{mb})$ & $-1.440 \pm 0.070$ \\
\hline$b_{1}$ & $-0.458 \pm 0.017$ & $c_{4}(\mathrm{mb})$ & $0.2817 \pm 0.0064$ \\
\hline$b_{2}$ & $-0.545 \pm 0.007$ & $d_{1}$ & -0.5 \\
\hline$b_{3}$ & 2 & $d_{2}$ & -0.585 \\
\hline$s_{l}\left(\mathrm{GeV}^{2}\right)$ & 1.0 & $d_{3}$ & 2 \\
\hline$s_{h}\left(\mathrm{GeV}^{2}\right)$ & $28.9 \pm 5.4$ & & \\
\hline
\end{tabular}

Tabela 3.1: Valor dos parâmetros que figuram nas parametrizações (3.54) e (3.55) cotados nas referências indicadas.

Como pode ainda ser observado, aparentemente nossos resultados a altas energias, em todos os casos, parecem não ser compatíveis com um comportamento tipo Froissart, isto é proporcional a $\ln ^{2}(s)$, apresentado pelas duas parametrizações acima. Todavia, constatamos que (em colisões $p p$ por exemplo) acima de $\sqrt{s} \sim 6 \mathrm{GeV}$ nossas curvas GRV98, CTEQ6L e EHKQS (curvas pontilhada, tracejada e sólida na Figura 3.5) também podem ser descritas pelas seguintes parametrizações à la PDG (equação (3.54), com $s_{l}=s_{h}=1 \mathrm{GeV}^{2}$ ) e à la $\mathrm{BH}$ (equação $(3.55)$, com $m=0.938 \mathrm{GeV}$ ) respectivamente:

$$
\widetilde{\sigma}_{C T E Q 6 L}^{G R V 98}(s)=(27.9 \pm 0.3)+a_{1} s^{b_{1}}-a_{2} s^{b_{2}}+(0.2152 \pm 0.0015) \ln ^{2}(s),(3.56)
$$




$$
\begin{aligned}
\tilde{\sigma}^{E H K Q S}(s) & =(30.5 \pm 0.8)+a_{1} s^{b_{1}}-a_{2} s^{b_{2}}+(0.2014 \pm 0.0035) \ln ^{2}(s), \\
\tilde{\sigma}_{C T E Q 6 L}^{G R V 98}(s) & =(29.74 \pm 0.11)+49.2143 s^{d_{1}}-39.7501 s^{d_{2}}-(0.252 \pm 0.004) \ln (s) \\
& +(0.2230 \pm 0.0004) \ln ^{2}(s) \\
\tilde{\sigma}^{E H K Q S}(s) & =(18.2 \pm 0.5)+49.2143 s^{d_{1}}-39.7501 s^{d_{2}}+(1.80 \pm 0.08) \ln (s) \\
& +(0.1445 \pm 0.0026) \ln ^{2}(s)
\end{aligned}
$$

onde os parâmetros $a_{1}, b_{1}, a_{2}, b_{2}, d_{1}$ e $d_{2}$ são os mesmos apresentados na Tabela 3.1.

Apesar disso, mesmo com as incertezas apresentadas na Tabela 3.1, as parametrizações empregadas em nossos resultados conduzem a um comportamento muito diferente para essas seções de choque, em relação aqueles preditos pelas equações (3.54) e (3.55). Eles sugerem que o crescimento dessas seções de choque com a energia é mais modesto, especialmente quando os efeitos não lineares são considerados. Evidentemente, a altas energias, os coeficientes dos termos $\ln s$ e $\ln ^{2}(s)$ em todas essas parametrizações são muito importantes e determinam o crescimento dessas seções de choque com a energia. De fato, o crescimento da seção de choque hadrônica a altas energias ainda é um problema em aberto [257], que demanda uma investigação (teórica e experimental) mais profunda.

A Figura 3.7 mostra os nossos resultados para as seções de choque inelásticas $p p$ e $\bar{p} p$. Os dados experimentais incluídos vão desde os resultados mais recentes, da colaboração Pierre Auger [220] e do LHC [217, 218,258-260], até os dados mais antigos, acessíveis a partir do Particle Data Group [216], onde os dados experimentais para esta seção de choque foram considerados como a diferença (a nível de medidas experimentais) $\sigma_{\text {inel }}^{p p(\bar{p})}(s)=\sigma_{\text {tot }}^{p p(\bar{p})}(s)-\sigma_{e l}^{p p(\bar{p})}(s)$.

Nos três casos (linhas sólida, tracejada e pontilhada) a descrição desses dados experimentais por nosso modelo não é boa. Como apontado acima, os parâmetros do presente modelo foram fixados apenas fazendo uso dos dados experimentais das seções de choque totais $p p$ e $\bar{p} p$. Esse ajuste determina apenas o comportamento da parte imaginária da função eiconal com a energia e, como consequência, não fornece uma 


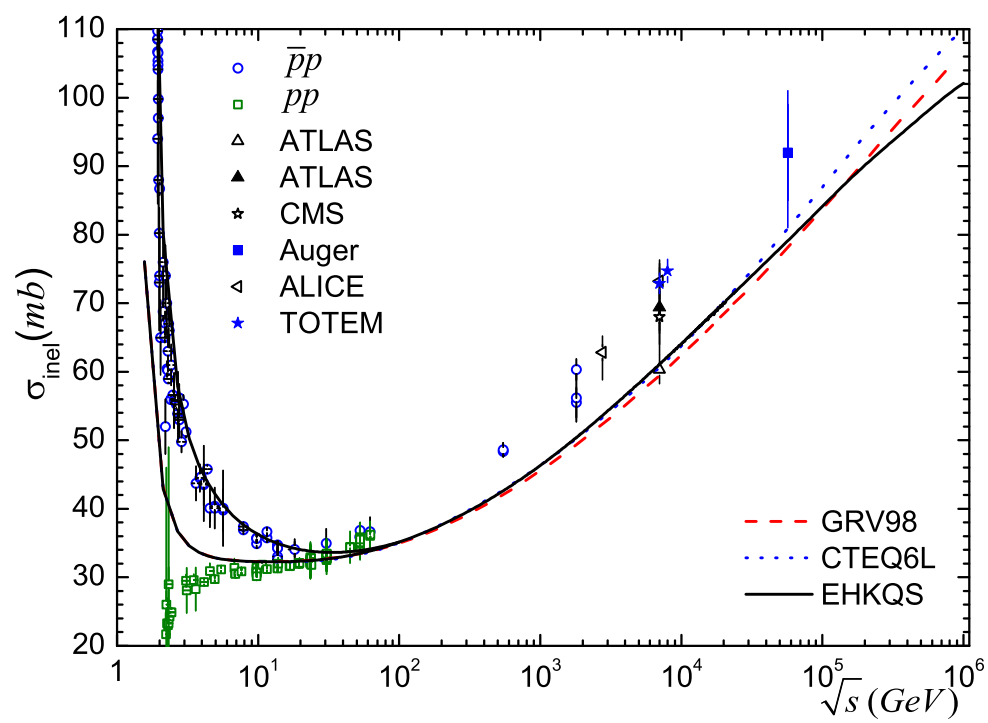

Figura 3.7: Seção de choque inelástica $p p$ e $p \bar{p}$ As linhas sólida, tracejada e pontilhada mostram o resultado do modelo eiconal (equação (3.29)) nos regimes não linear e linear. Os dados experimentais são das referências [217, 218, 220, 258-260].

descrição satisfatória dessas seções de choque. É importante notar, entretanto, que os modelos eiconais convencionais possuem o mesmo problema e também não são capazes de produzir uma descrição satisfatória desses dados experimentais [222, 245-251].

Afim de modificar este comportamento alguns modelos alternativos inspirados em QCD vem sendo propostos. Por exemplo, Luna e colaboradores [261] introduziram uma massa dinâmica para os gluons na região do infravermelho no cálculo de quantidades frontais nos espalhamentos $p p$ e $p \bar{p}$ que (segundo os autores) permite descrever com sucesso as quantidades $\sigma_{\text {tot }}^{p p(\bar{p})}, \rho$ (a razão entre a parte imaginária e real da amplitude frontal de espalhamento), o "slope nuclear", $B$ e a seção de choque diferencial, $d \sigma_{e l}^{p p(\bar{p})}(s, t) / d t$, em muito boa concordância com os dados experimentais (pelo menos até $\sqrt{s}=1.8 \mathrm{TeV}$ ). Por outro lado, Gribov e colaboradores [33] propuseram a inclusão de um corte dependente da energia na região de baixos momentos transversais que, efetivamente, simula os efeitos da saturação. Outra abordagem interessante assume a ressoma de gluons emitidos com baixíssimos momentos transversais de modo a atenuar o crescimento da seção de choque devido ao aumento do número de colisões 
gluon-gluon a altas energias [255]. Como deve ter sido notado, o modelo por nós utilizado não utiliza nenhuma dessas abordagens.

Assim como os demais modelos eiconais mencionados anteriormente, por outro lado, nosso modelo, dada a simplicidade que entendemos interessante resguardar, não inclui processos difrativos. No entanto, como apontado em [262], as medidas recentes da componente difrativa da seção de choque inelástica $p p$ realizadas no LHC indicam que a taxa de eventos difrativos em colisões inelásticas, estimadas a partir da distribuição de pseudorapidez de partículas carregadas, é $\sigma_{S D} / \sigma_{\text {inel }} \simeq 0.20$ para processos unicamente difrativos ("single diffractive") e $\sigma_{D D} / \sigma_{\text {inel }} \simeq 0.12$ para processos duplamente difrativos ("double diffractive"). Claramente a inclusão de uma componente difrativa seria desejável e necessária em nosso modelo de modo a possibilitar uma melhor concordância com os dados experimentais de $\sigma_{\text {inel }}^{p p}$, entretanto, sua inclusão requereria uma abordagem muito mais complexa do que a utilizada nesta tese.

Embora complementar, é ainda importante mencionar que, a Figura 3.7 mostra que o papel dos efeitos da saturação de pártons a altíssimas energias e indica que o uso de outras abordagens ou a inclusão de processos difrativos é realmente necessário.

Neste momento gostaríamos de chamar atenção para os dados mais recentes do LHC [217,218]: eles fornecem informações muito valiosas sobre a produção múltipla de partículas a altas energias e possibilitam uma melhora no entendimento teórico acerca da dinâmica partônica a nível perturbativo e não perturbativo. Eles também mostram a necessidade de ajustes ou até mesmo de reformulações de hipóteses empregadas nos modelos que se propõem a estabelecer o comportamento das seções de choque hadrônicas com a energia.

Igualmente, e de forma complementar, os dados experimentais recentemente obtidos pelas colaborações HiRes [219] e Pierre Auger [220], têm permitido um aprofundamento no entendimento sobre a natureza das partículas produzidas a energias muito maiores que as disponíveis nos aceleradores de partículas atuais e, portanto, vêm estimulando muitas discussões entre as comunidades de físicos de raios cósmicos e de aceleradores sobre questões comuns nessas áreas do conhecimento. 
A dependência energética da seção de choque total hadrônica é, provavelmente, uma das questões mais importantes para a física de raios cósmicos. As regiões relevantes do espaço de fase para o desenvolvimento dos chamados "air showers", isto é, "chuveiros atmosféricos", nos quais partículas são criadas a partir de múltiplas colisões do próton projétil com a atmosfera terrestre, não são diretamente acessíveis nos aceleradores atuais e, por conta disso, as descrições e interpretações dos dados experimentais da física de raios cósmicos a altíssimas energias dependem de maneira crucial das predições oriundas de modelos fenomenológicos [263-268].

Por essa razão é muito interessante testar o alcance de modelos hadrônicos por nós utilizados e verificar se eles permitem uma descrição satisfatória dos dados experimentais obtidos nesses experimentos. Nesse sentido, como uma trajetória que julgamos um tanto que natural, estudamos também o comportamento da seção de choque prótonAr com a energia, cujos valores experimentalmente medidos se estendem desde poucos GeV's até dezenas de TeV's. Utilizando as expressões derivadas na seção 3.3 nos foi possível estender a aplicação de nosso modelo ao estudo da seção de choque inelástica próton-Ar.

Nesta seção mostrou-se que a seção de choque inelástica total para uma colisão próton-núcleo, $\sigma_{\text {inel }}^{p A}$, pode ser obtida, na aproximação eiconal, a partir da seção de choque total nucleon-nucleon, dada por

$$
\sigma_{\text {inel }}^{h A}\left(E_{l a b}\right)=\int d^{2} \vec{b}\left[1-e^{-\sigma_{t o t}^{h N} T(\vec{b})}\right]
$$

onde $E_{l a b}$ relaciona-se com a energia do centro de massa nucleon-nucleon através da equação (3.45).

A seção de choque $\sigma_{\text {inel }}^{h A}\left(E_{l a b}\right)$ leva em consideração todos os canais de produção de partículas, isto é, que incluem também a contribuição de processos onde o núcleo se "quebra" em diversos nucleons e/ou fragmentos nucleares. No entanto, os experimentos dedicados a medir a seção de choque inelástica próton-Ar têm acesso apenas à fração da seção de choque inelástica onde são produzidas novas partículas [269]. 
Para se obter a fração da seção de choque inelástica referente apenas à produção de novas partículas em uma colisão próton-núcleo, isto é, a seção de choque (inelástica) de produção, $\sigma_{\text {prod }}^{p A}$, deve-se subtrair a contribuição dos eventos onde o núcleo se quebra e se fragmenta em outras partículas, da seção de choque inelástica total. Após esse procedimento tem-se que [270]

$$
\sigma_{\text {prod }}^{h A}\left(E_{l a b}\right)=\int d^{2} \vec{b}\left[1-e^{-\sigma_{\text {inel }}^{h N} T(\vec{b})}\right]
$$

A Figura 3.8 mostra os resultados para seção de choque de produção próton-Ar para as três distribuições de partons utilizadas anteriormente em nossos cálculos das seções de choque inelásticas $p p$, mostradas na Fig. (3.7). Em todos os casos a função de perfil nuclear, $T(b)$, foi normalizada a $A=A_{a r}=14,5$ levando-se em consideração a densidade de nucleons na atmosfera terrestre dada por

$$
\rho(b, z)=\frac{\rho_{0}}{1+\exp \left[\left(r-R_{A}\right) / a_{0}\right]},
$$

onde $r=\sqrt{b^{2}+z^{2}}, R_{A}=1,19 A^{1 / 3}-1,61 A^{-1 / 3}$ fm e $a_{0}=0,75 \mathrm{fm}[271]$.

Como pode ser observado uma vez mais, a descrição dos dados experimentais da seção de choque inelástica $p-A r$ a partir de nosso modelo não é boa. No entanto, os resultados obtidos (mesmo com a evolução não linear) a altas energias através da (simples) abordagem adotada possuem a mesma qualidade das predições advindas de simulações de Monte Carlo [282,283]. É interessante notar, porém, que nossos resultados favorecem um crescimento moderadamente mais lento para a seção de choque próton-Ar a altas energias, como indicado pela medida mais recente efetuada pela colaboração Pierre Auger [220]. Em nossa opinião, esses resultados terão implicações sobre medidas vindouras do LHC (cuja primeira análise também indicou um crescimento da seção de choque hadrônica ligeiramente menor do que o esperado por diversos modelos) e, certamente testarão as hipóteses, dinâmicas e predições dos modelos existentes. 


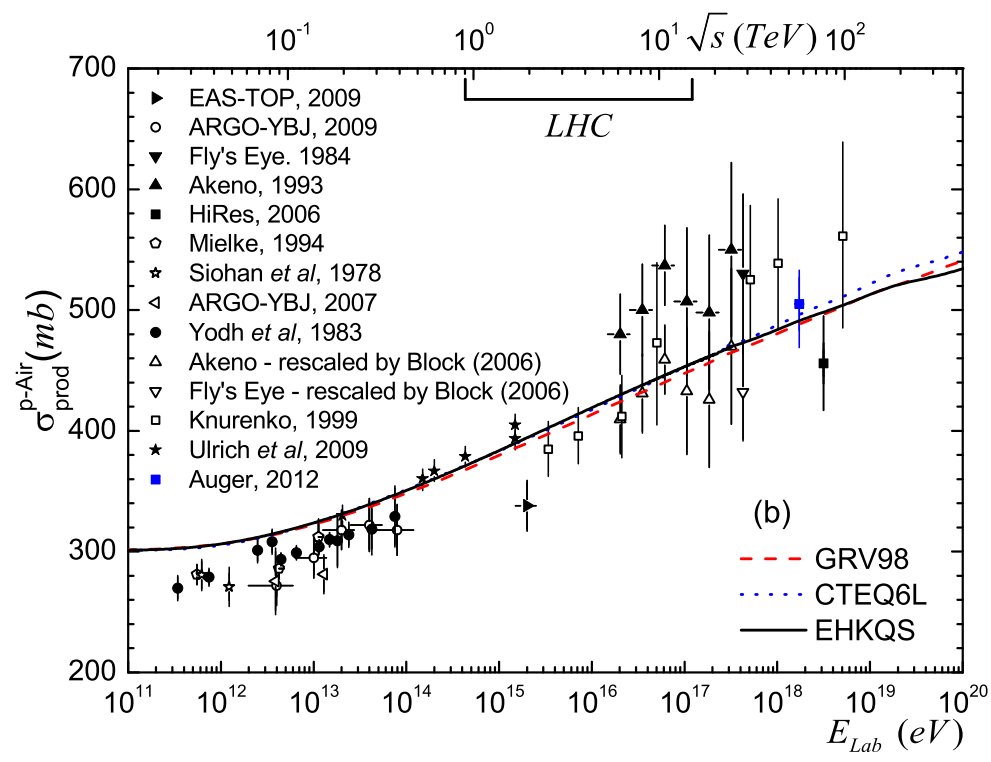

Figura 3.8: Seção de choque inelástica de produção próton-Ar. As linhas sólida, tracejada e pontilhada mostram o resultado do modelo eiconal nos regimes não linear e linear. Os dados experimentais são das referências [220, 224, 244, 272 281].

\subsubsection{Resultados para as seções de choque de fotoprodução}

A segunda escolha para testar o modelo por nós utilizado foram as seções de choque de fotoprodução fóton-próton $(\gamma p)$ e fóton-fóton $(\gamma \gamma)$. A seção de choque para estes processos pode ser derivada a partir da amplitude de espalhamento frontal $p p$ fazendo-se uso da dominância de mésons vetoriais e do modelo aditivo de quarks, com a introdução da probabilidade de que o fóton interaja como um hádron $\left(P_{\text {had }}^{\gamma p(\gamma)}\right)[224,254,255,261,284]$.

Assumindo-se, no espírito da dominância de mésons vetoriais, que a altas energias o fóton se comporte como um estado hadrônico composto de dois quarks, a seção de choque $\gamma p$ é obtida após as substituições $\sigma \rightarrow \frac{2}{3} \sigma$ e $\mu \rightarrow \sqrt{\frac{3}{2}} \mu$ nas duas componentes (soft e pQCD) da função eiconal (equação 3.47), e pode ser escrita como

$$
\sigma_{\text {tot }}^{\gamma p}(s)=2 P_{h a d}^{\gamma p} \int\left[1-e^{-\chi_{I}^{\gamma p}(b, s)} \cos \left(\chi_{R}^{\gamma p}(b, s)\right)\right] d^{2} \vec{b} .
$$


Para se obter a seção de choque $\gamma \gamma$ aplica-se o mesmo procedimento acima realizandose, nesse caso, as substituições $\sigma \rightarrow \frac{4}{9} \sigma$ e $\mu \rightarrow \sqrt{\frac{9}{4}} \mu$ nessas componentes:

$$
\sigma_{\text {tot }}^{\gamma \gamma}(s)=2 P_{h a d}^{\gamma \gamma} \int\left[1-e^{-\chi_{I}^{\gamma \gamma}(b, s)} \cos \left(\chi_{R}^{\gamma \gamma}(b, s)\right)\right] d^{2} \vec{b}
$$

$\operatorname{com} P_{h a d}^{\gamma \gamma}=\left(P_{h a d}^{\gamma p}\right)^{2}$

A formulação mais simples do modelo da dominância de mésons vetoriais leva em consideração apenas os mésons vetoriais mais leves e, nesse caso, tem-se que $P_{\text {had }}^{\gamma p}=$ $\sum_{V=\rho, \omega, \varphi} \frac{4 \pi \alpha_{e m}}{f_{V}^{2}}$, onde $\alpha_{e m}(\sim 1 / 137)$ representa a constante de acoplamento da QED e $f_{V}^{2}$ o acoplamento $\gamma V$. Nesta tese, no entanto, considerou-se $P_{h a d}^{\gamma p}$ como um parâmetro livre a ser fixado pelos dados experimentais a baixas energias.

As Figuras 3.9 (a) e 3.9 (b) mostram o resultado para as seções de choque totais $\gamma p$ e $\gamma \gamma$, respectivamente dadas por (3.63) e (3.64). Os dados experimentais são das referências [216,285-295]. Em ambas as figuras a probabilidade de que o fóton interaja como um hádron foi fixada em $P_{h a d}^{\gamma p}=1 / 221$.
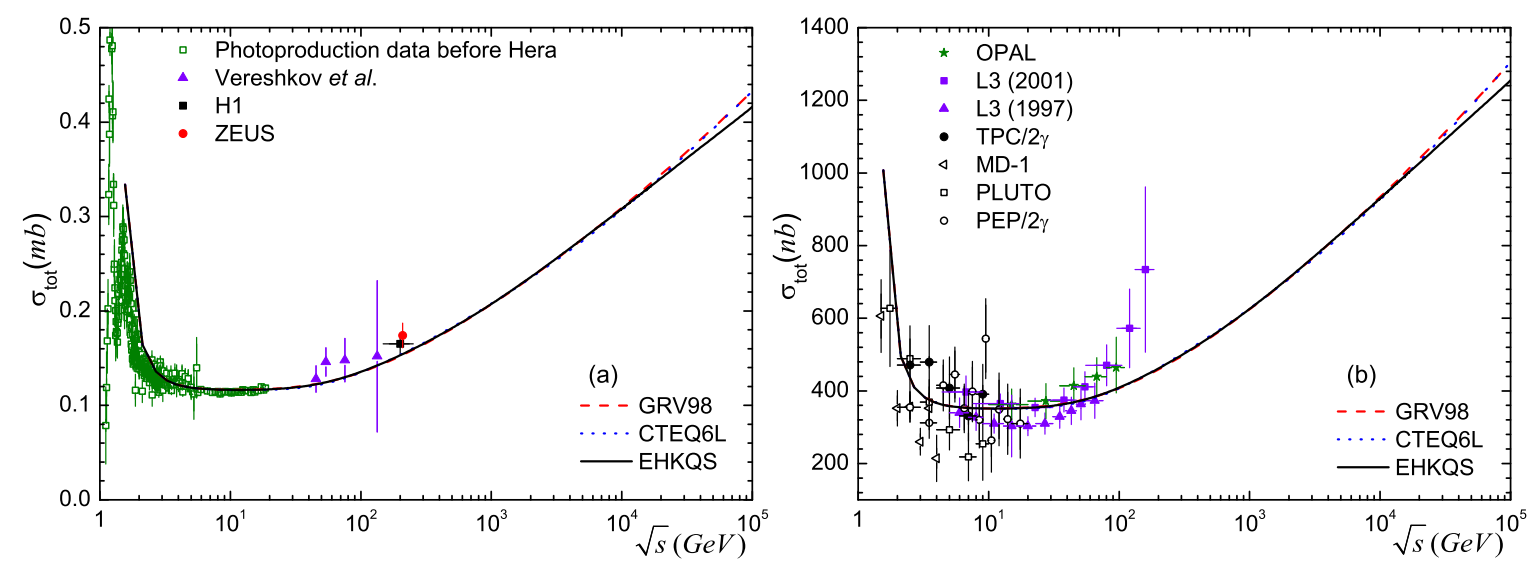

Figura 3.9: (a) Seção de choque total $\gamma p$. As linhas sólida, tracejada e pontilhada representam os resultados do nosso modelo (equação (3.63)) para diferentes distribuições de pártons. Os dados experimentais são das referências [216, 285-287]. (b) Resultados para a seção de choque total $\gamma \gamma$. As linhas sólida, tracejada e pontilhada representam os resultados do nosso modelo (equação (3.64)) para diferentes distribuições de pártons. Os dados experimentais são da referência [288-295].

Considerando-se que a dinâmica e o teor de crescimento das seções de choque 
de fotoprodução foram determinadas a partir dos mesmos parâmetros utilizados nas seções de choque $p p$ e $\bar{p} p$ e pelos "pesos" introduzidos através da dominância de mésons vetoriais e do modelo aditivo de quarks, a concordância com os dados experimentais (em ambos os casos) é razoável.

O modelo, entretanto, subestima os últimos pontos experimentais da seção de choque total $\gamma p$ e não é capaz de descrever os dados experimentais a partir de $\sqrt{s} \sim 100$ $\mathrm{GeV}$ para a seção de choque $\gamma \gamma$. Conforme discutido anteriormente, este também é um problema comum a outros modelos eiconais propostos na literatura.

Por fim, o fato de termos obtido um sucesso razoável no estudo das seções de choque totais hadrônicas e fotônicas utilizando o simples modelo apresentado, nos encorajou a estudar outros observáveis, também incluindo os efeitos da saturação de pártons. O próximo capítulo será dedicado ao estudo da produção de partículas nas regiões central e, principalmente, frontal de rapidez. 


\section{Capítulo 4}

\section{Produção inclusiva de hádrons leves e pesados com efeitos de saturação}

Esse capítulo é dedicado ao estudo da produção de partítulas considerando-se diversos sistemas de colisão (próton-próton, $p p$, próton-núcleo, $p A$ e núcleo-núcleo, $A A$ ) e em diversas regiões cinemáticas levando-se em consideração os efeitos de saturação de pártons através da teoria efetiva do CGC.

Como ponto de partida, ele se inicia com a apresentação das abordagens empregadas no desenvolvimento dos estudos sobre a produção de partículas leves e pesadas das subseções seguintes, a chamada "fatorização $k_{T}$ " e o "formalismo híbrido". Nestas duas abordagens, os efeitos de saturação são incluídos através da amplitude de espalhamento dipolo-hádron, apresentada no Capítulo 2. A seguir, foca-se na produção de partículas na região frontal de rapidez. Primeiramente apresenta-se um estudo sobre a produção líquida de bárions, isto é, o número de bárions menos o número de antibárions, $\Delta B \equiv B-\bar{B}$ e, posteriormente, um outro estudo sobre a produção de mésons $D$. Em ambos os casos estuda-se, entre outros observáveis, a distribuição de rapidez, $y$, e de $x$-Feynman, $x_{F}$, dessas partículas. Uma análise sobre o comportamento do momento transversal médio com a energia e a rapidez, $\left\langle p_{T}(y, \sqrt{s})\right\rangle$, de partículas 
carregadas produzidas em colisões $p p$ e $p A$ encerra o estudo sobre a produção de partículas nesta região cinemática. Por fim, apresenta-se um estudo sobre as distribuições de rapidez e de densidade de partículas carregadas na região central de rapidez. Nosso principal objetivo será fornecer uma primeira estimativa (qualitativa) de como as correções em $\alpha_{s}$ recentemente incluídas no formalismo da fatorização $k_{T}$ alteram esses observáveis.

\subsection{Produção de partítulas na teoria efetiva do CGC: a fatorização $k_{T}$ e o formalismo híbrido}

Nesta subseção sumariza-se como a produção de partículas em colisões a altas energias é calculada segundo a teoria efetiva do CGC. Ao contrário do capítulo anterior, onde a cinemática de processos de dois corpos para dois corpos, $i j \rightarrow k l$, foi utilizada, na teoria efetiva do CGC, utilizamos aqui a cinemática de dois corpos para um, detalhada no apêndice da referência [73]. Neste caso, considerando-se a produção inclusiva de um párton de sabor $f\left(q, \bar{q}\right.$ ou $g$ ) com momento transversal $q_{T}$ e rapidez $y$, projétil e alvo são provados com as frações de momento $x_{1,2}=\left(q_{T} / \sqrt{s}\right) e^{ \pm y}$, respectivamente, com $\sqrt{s}$ sendo a energia do centro de massa do espalhamento.

Obviamente, partículas podem ser produzidas em todo o espaço de fase disponível numa dada colisão, no entanto, projétil e alvo são provados em regiões cinemáticas diferentes, dependendo do setor do espaço de fase considerado. A teoria efetiva do CGC fornece duas abordagens distintas, porém complementares, para a produção de partículas dependendo da região cinemática provada. Em ambas as abordagens, no entanto, os hádrons são descritos através da distribuição não integrada de gluons (apropriada para cada uma das abordagens mencionadas) quando provados no regime de grandes densidades partônicas e pequenos valores de $x_{1,2}$, como será visto a seguir.

Caso as partículas sejam produzidas na região central de rapidez, $|y| \lesssim 2$, em 
colisões a altas energias, projétil e alvo são provados com pequenos valores de $x_{1,2}$ $(\lesssim 0,01)$. Nesta situação, conforme discutido anteriormente, a função de onda dos hádrons colidentes é dominada por gluons e os efeitos não lineares da QCD passam a ser importantes (tanto no projétil quanto no alvo) e não podem ser desprezados.

$\mathrm{Na}$ teoria efetiva do CGC, a dinâmica da produção de partículas nesta região cinemática é descrita fazendo-se uso do chamado formalismo da "fatorização $k_{T}$ ", onde os hádrons colidentes são caracterizados através da seguinte distribuição não integrada de gluons ${ }^{1}$,

$$
\phi_{h_{i}}(\boldsymbol{k}, y)=\frac{C_{F}}{\alpha_{s}(2 \pi)^{3}} \int d^{2} b d^{2} r e^{-i \boldsymbol{k} \cdot \boldsymbol{r}} \nabla_{r}^{2} \mathcal{N}_{h_{i}}^{G}(\boldsymbol{r}, \boldsymbol{b}, y)
$$

onde $\mathcal{N}_{h_{i}}^{G}(\boldsymbol{r}, \boldsymbol{b}, y)$ representa a amplitude de espalhamento frontal dipolo-hádron $h_{i}$ para um dipolo de tamanho transversal $\boldsymbol{r}$ formado por gluons, $\boldsymbol{b}$ representa o parâmetro de impacto do espalhamento, e $\phi_{h_{i}}(\boldsymbol{k}, y)$ representa uma função adimensional que corresponde ao número de gluons por unidade área transversal e por unidade de momento transversal no hádron $h_{i}$.

O formalismo da fatorização $k_{T}$ para a produção de gluons na região central de rapidez foi proposto em [33], e posteriormente derivado nas aproximações de $\log (1 / x)$ dominante (isto é, onde apenas divergências do tipo $\alpha_{s} \log (1 / x)$ são ressomadas) e de acoplamento fixo ( $\alpha_{s}$ constante) [74] considerando-se o espalhamento de um sistema partônico diluído por outro denso (uma colisão $p A$, por exemplo, onde a escala de saturação do núcleo é muito maior que a escala de saturação do próton, $Q_{s, A} \gg Q_{s, p}$ ).

Nessa abordagem, a seção de choque invariante para a produção inclusiva de gluons com rapidez $y$ e momento transversal $k_{T}$ a partir da colisão de dois hádrons, $h_{1,2}$, é dada por

$$
\frac{d^{3} \sigma}{d^{2} k_{T} d y}=\frac{2}{C_{F}} \frac{1}{\boldsymbol{k}^{2}} \int d^{2} q \alpha_{s} \phi_{h_{1}}(\boldsymbol{q}, y) \phi_{h_{2}}(\boldsymbol{k}-\boldsymbol{q}, Y-y)
$$

\footnotetext{
${ }^{1}$ Variáveis em negrito denotam vetores no plano transversal; $\boldsymbol{k}=\left(k^{1}, k^{2}\right)$, por exemplo.
} 
onde $Y$ é o intervalo total de rapidez da colisão, $C_{F}=\left(N_{c}^{2}-1\right) / 2 N_{c}$, com $N_{c}(=3)$ representando o número de cores da QCD.

É importante notar que a equação acima é simétrica na troca $\boldsymbol{q} \leftrightarrow \boldsymbol{k}-\boldsymbol{q}$, devido à simetria existente na troca projétil $\leftrightarrow$ alvo no processo de espalhamento para o caso de colisões envolvendo hádrons idênticos. No caso de uma colisão $p A$, por outro lado, deve-se também efetuar a troca $y \leftrightarrow Y-y$.

A fatorização $k_{T}$ vem sendo aplicada com sucesso na descrição de dados experimentais medidos em colisores de partículas. Nas referências $[134,166,296]$ mostra-se que este formalismo é capaz de descrever, por exemplo, a distribuição de rapidez de partículas produzidas em colisões de íons pesados, o chamado "fator de modificação nuclear", $R_{p A}$, de partículas carregadas em colisões $p A$, que é definido como a razão entre as seções de choque de produção de partículas carregadas em colisões $p A$ e $p p$ (essa última quantidade escalonada pelo número de participantes em uma colisão, $N_{\text {part }}{ }^{2}$ ), a dependência do momento transversal médio com a energia medido em colisões $p p$ e, por fim, a dependência com a energia da multiplicidade de hádrons carregados na região de rapidez central, $y \sim 0$, medida em colisões $p p$ e $A A$.

Como mencionado anteriormente, a equação (4.2) foi deduzida assumindo-se uma constante de acoplamento fixa. Todavia, correções em $\alpha_{s}$ para a equação (4.2) foram calculadas recentemente em [76] para rapidez nula e uma conjectura (a priori válida para todo o espaço de fase) foi proposta para a seção de choque de produção de gluons. A validade ou não desta conjectura é uma questão em aberto atualmente. Essa e outras questões relacionadas ao formalismo da fatorização $k_{T}$ serão exploradas em maiores detalhes na seção 4.5, onde apresenta-se um estudo qualitativo sobre o impacto das correções em $\alpha_{s}$ incluídas na equação (4.2) na distribuição de rapidez de partículas carregadas e na dependência com a energia da multiplicidade de hádrons carregados produzidos na região central de rapidez em colisões $p p, p A$ e $A A$.

Alternativamente, quando os hádrons são produzidos na região frontal de rapidez,

\footnotetext{
${ }^{2}$ Essa não é a única quantidade utilizada na definição de $R_{p A}$. Como pode ser visto nas referências [78,166,297], também é possível utilizar, por exemplo, o número de colisões binárias, $N_{\text {coll }}$, em colisões $p A$ na normalização dessa razão.
} 
$|y| \gg 2$, o projétil passa a ser provado em sua região de fragmentação, isto é na região de grandes valores de $x_{1}(\gg 0,01)$, enquanto o alvo é provado em valores ainda menores de $x_{2}(\ll 0,01)$, cada vez mais dentro do regime de saturação. Como resultado, tem-se uma colisão altamente assimétrica onde o projétil encontra-se no regime diluído e o alvo no regime denso. Neste caso, o formalismo da fatorização $k_{T}$ falha em capturar a contribuição dominante do processo de espalhamento, que também pode ocorrer com um quark de valência do projétil, por exemplo.

O chamado "formalismo híbrido" da teoria efetiva do CGC, proposto em [73], é mais indicado para estudar a produção de partículas nesta região cinemática. Nesse formalismo, os graus de liberdade com altos valores de $x_{1}$ do projétil (usualmente um próton) são descritos pelas PDFs usuais, $f\left(x, \mu^{2}\right)$, onde a dependência com a escala $\mu^{2}$ obedece as equações DGLAP, apresentadas no capítulo 1. O alvo, por outro lado, é descrito pela seguinte distribuição não integrada de gluons,

$$
\widetilde{\mathcal{N}}_{F, A}\left(x, p_{T}\right)=\int d^{2} r e^{i \overrightarrow{p_{T}} \cdot \vec{r}}\left[1-\mathcal{N}_{\mathcal{F}, \mathcal{A}}(x, r)\right]
$$

onde $r$ denota o tamanho transversal do dipolo, $\mathcal{N}_{\mathcal{F}}(x, r)$ e $\mathcal{N}_{\mathcal{A}}(x, r)$ representam as amplitudes de espalhamento dipolo-hádron, escritas na representação fundamental (onde os constituintes do dipolo são um par quark-antiquark) e na representação adjunta (onde o dipolo é formado por um par de gluons), respectivamente.

A amplitude $\mathcal{N}_{F}(x, r)$ acima pode ser obtida através da amplitude $\mathcal{N}_{A}(x, r)$ efetuandose um reescalonamento da escala de saturação $Q_{s, F}^{2}=\left(C_{F} / C_{A}\right) Q_{s, A}^{2}$, onde $C_{F} / C_{A}=$ 4/9 [205], onde $C_{F}=\left(N_{c}^{2}-1\right) / 2 N_{c}$ e $C_{A}=N_{c}$, denotam, respectivamente, os operadores de Casimir na representação fundamental e adjunta do grupo de simetria da QCD, o $S U\left(N_{c}\right)$, com $N_{c}=3$.

Assim como a distribuição não integrada de gluons utilizada na fatorização $k_{T}$, $\phi_{h_{i}}(\boldsymbol{q}, y)$, as distribuições $\widetilde{\mathcal{N}}_{F, A}\left(x, p_{T}\right)$ carregam toda a informação sobre o espalhamento hadrônico e, portanto, sobre os efeitos não lineares e quânticos na função de onda hadrônica. 
Neste formalismo, a seção de choque invariante para a produção inclusiva de hádrons do tipo $h$ que carregam uma fração de momento $z$ em um espalhamento envolvendo dois hádrons, $h_{1,2}$ é obtida através da convolução das distribuições $f\left(x, \mu^{2}\right)$ e $\widetilde{\mathcal{N}}_{F, A}\left(x, p_{T}\right)$ com uma função de fragmentação correspondente, $D_{h / f}\left(z, \mu^{2}\right)$, que converte o párton $f(=q, \bar{q})$ no hádron $h$ :

$$
\begin{aligned}
\frac{d N_{h}}{d y d^{2} p_{T}}= & \frac{K(y)}{(2 \pi)^{2}} \int_{x_{F}}^{1} \frac{d z}{z^{2}}\left[\sum_{f=q, \bar{q}} x_{1} f_{f / p}\left(x_{1}, \mu^{2}\right) \widetilde{\mathcal{N}}_{F}\left(q_{T}, x_{2}\right) D_{h / f}\left(z, \mu^{2}\right)\right. \\
& \left.+x_{1} f_{g / p}\left(x_{1}, \mu^{2}\right) \widetilde{\mathcal{N}}_{A}\left(q_{T}, x_{2}\right) D_{h / g}\left(z, \mu^{2}\right)\right]
\end{aligned}
$$

Na equação acima, $p_{T}, y$ e $x_{F}=\left(p_{T} / \sqrt{s}\right) e^{y}$ representam, respectivamente, o momento transversal, a rapidez e o $x$-Feynman do hádron produzido e $q_{T}=p_{T} / z$ representa o momento transversal do párton interagente. O fator $K(y)$ leva em consideração correções de mais altas ordem e, de forma efetiva, outros efeitos dinâmicos não incluídos na formulação do CGC.

No formalismo híbrido o processo de hadronização, descrito pelas funções de fragmentação, pode ser tratado utilizando-se a teoria perturbativa padrão da QCD, da mesma maneira que as divergências colineares são ressomadas pelas equações DGLAP. Ao contrário, uma vez que a ressoma de divergências colineares não está inclusa no formalismo da fatorização $k_{T}$, o uso de tais funções de fragmentação, nesse caso, não é inteiramente justificado.

A equação (4.4) pode ser entendida de uma maneira simples e intuitiva. O primeiro termo corresponde a um espalhamento elástico de um quark/antiquark $\left(f_{q / \bar{q}}\right)$ presente no projétil com o alvo que se encontra no regime denso, descrito pela física do CGC. O párton incidente, inicialmente de momento transversal nulo, adquire então momento transversal da ordem da escala de saturação do alvo após sofrer múltiplos espalhamentos ao atravessá-lo. O segundo termo possui a mesma interpretação, com a diferença de que a interação acontece com um gluon originário do projétil.

Assim como a fatorização $k_{T}$, o formalismo híbrido apresentado acima também 
vem sendo aplicado com sucesso. Na referência [298], mostrou-se que este formalismo é capaz de descrever as distribuições de rapidez da quantidade líquida de bárions nas energias do RHIC. Já na referência [299], mostrou-se que é possível obter uma boa descrição da distribuição de momento transversal e da distribuição de rapidez de partículas carregadas produzidas nas energias do RHIC e do LHC. Posteriormente, essa descrição foi aperfeiçoada na referência [300] fazendo-se uso de um tratamento melhorado da geometria nuclear em colisões hádron-núcleo.

Na próxima subseção, mostramos a aplicação desse formalismo no estudo da produção líquida de bárions em colisões $p p, p A$ e $A A$ na região de grande rapidez. Dentre outros resultados interessantes, mostramos que utilizando a amplitude de espalhamento de dipolo BUW, apresentada na seção 2.3.4, é possível melhorar a descrição da distribuição de momento transversal da produção líquida de bárions apresentada na referência [301].

\subsection{Produção líquida de bárions no formalismo do CGC}

Em colisões hadrônicas a produção de bárions ocorre nas regiões central $(|y| \lesssim 2)$ e frontal $(|y| \gg 2)$ de rapidez, conforme mencionado anteriormente. No primeiro caso os bárions são produzidos juntamente com os antibárions e o número líquido de bárions, $\Delta B$, é pequeno. Entretanto, na região frontal, os experimentos revelam uma produção majoritária de bárions [302-309] e, portanto, esse número líquido é grande.

A altas energias e grandes valores de rapidez esse fato experimental sugere que a produção de bárions nessa região ocorra devido a interação dos quarks de valência do projétil, que carregam uma grande fração de momento, com pártons do alvo (predominantemente gluons) que, ao contrário, carregam uma pequena fração de momento. Os bárions assim produzidos são denominados "dominantes", uma vez que carregam uma grande fração de momento do projétil. 
Conforme apontado no capítulo 2, neste regime, espera-se a formação de um estado contendo uma alta densidade de pártons onde a dinâmica da QCD torna-se qualitativamente diferente da dinâmica no regime de curtas distâncias e de altos momentos transferidos, o Condensado de Vidro de Cor [33-58]. Diferentemente do formalismo utilizado no capítulo 3, nesse cenário, os efeitos da saturação de pártons estão contidos na amplitude de espalhamento dipolo de cor-hádron, construída de maneira fenomenológica, como discutido no capítulo 2.

Nessas condições, a interação entre um quark (antiquark) de valência do projétil com os campos de cor do projétil pode ser descrita fazendo-se uso do formalismo híbrido. Mais especificamente, messa abordagem, a seção de choque diferencial para a produção líquida de bárions, $\Delta B$, com momento transversal $p_{T}$ e rapidez, $y$, é dada pelo primeiro termo da equação (4.4),

$$
\frac{d N}{d y d^{2} p_{T}}=\frac{K(y)}{(2 \pi)^{2}} \int_{x_{F}}^{1} \frac{d z}{z^{2}} \sum_{f=q, \bar{q}} x_{1} f_{f / p}\left(x_{1}, \mu^{2}\right) \widetilde{\mathcal{N}}_{F}\left(q_{T}, x_{2}\right) D\left(z, \mu^{2}\right)
$$

repetida aqui por completeza.

Na equação acima, $x_{1} f_{f / p}\left(x_{1}, \mu^{2}\right) \equiv x_{1} q_{v / p}\left(x_{1}, \mu^{2}\right)$ denota a distribuição de quarks de valência no projétil e a função de fragmentação da quantidade líquida de bárions é definida por:

$$
D\left(z, \mu^{2}\right) \equiv D_{\Delta B / q_{v}}\left(z, \mu^{2}\right)=D_{B / q_{v}}\left(z, \mu^{2}\right)-D_{\bar{B} / q_{v}}\left(z, \mu^{2}\right)
$$

onde as parcelas acima representam as funções de fragmentação de quarks (antiquarks) em bárions (antibárions), comumente utilizadas na literatura, $z\left(=E_{B} / E_{q_{v}}\right)$ representa a fração de energia do quark de valência que se fragmenta e que é carregada pelo bárion (antibárion) emergente, $B(\bar{B})$.

Em (4.5), as frações de momento dos quarks (antiquarks) de valência no projétil e dos gluons no alvo são $x_{1,2}=q_{T} e^{ \pm y} / \sqrt{s}$, onde $q_{T}=\sqrt{p_{T}^{2}+m^{2}} / z, x_{F}=\sqrt{p_{T}^{2}+m^{2}} e^{y} / \sqrt{s}$ e $m$ representam, respectivamente, o momento transversal do gluon no alvo, o $x$ - 
Feynman e a massa do bárion emergente.

A distribuição de gluons no alvo utilizada neste estudo, $\widetilde{\mathcal{N}}_{F}\left(q_{T}, x_{2}\right)$, é a principal diferença em relação à abordagem assumida nos estudos anteriores. Aqui ela é dada pela equação (4.3), onde o subscrito F indica que essa distribuição é calculada na representação fundamental.

É importante ressaltar que, em primeiro lugar, esta distribuição leva em consideração que os pártons do alvo possuem momentos transversais não nulos, fato este que foi ignorado no formalismo empregado nos estudos do capítulo anterior. Em segundo lugar, os efeitos da saturação de gluons encontram-se contidos na quantidade $\mathcal{N}(x, r)$ que, formalmente, resulta da solução de uma equação de evolução não linear, como as equações BK [44-47] ou JIMWLK [48-56]. Todavia uma solução analítica completa para uma dessas equações não lineares ainda não é conhecida e, por essa razão, inúmeros modelos fenomenológicos têm sido propostos (três destes modelos para $\mathcal{N}(x, r)$ foram apresentados nas seções 2.3 .3 e 2.3.4).

Conforme mencionado no capítulo 2, em geral assume-se que a amplitude de dipolo quark-hádron, $\mathcal{N}(x, r)$, pode ser modelada segundo uma função eiconal (vide equação (2.27)), onde a principal diferença entre os diversos modelos encontrados na literatura vem do comportamento predito pela chamada "dimensão anômala", $\gamma\left(x, r^{2}\right)$, responsável por determinar quão rápido ocorre a transição do regime de saturação (não linear) para o regime diluído (linear). Os modelos GBW [113] e BUW [205], para os quais essas quantidades são dadas por,

$$
\begin{aligned}
\gamma\left(x, r^{2}\right)_{G B W} & =1 \\
\gamma\left(x, r^{2}\right)_{B U W} & =\gamma_{s}+\left(1-\gamma_{s}\right) \frac{\left(\omega^{a}-1\right)}{\left(\omega^{a}-1\right)+b},
\end{aligned}
$$

onde $\omega \equiv 1 /\left(r Q_{s}\right), \gamma_{s}=0.628, a=2.82$ e $b=168$, foram considerados neste estudo.

O modelo GBW empregado nas referências [298,301], onde a produção líquida de bárions também foi estudada, também foi incluso nesse estudo, motivado pela possibilidade de comparação de nossos resultados com aqueles previamente apresentados 
nessas referências. No primeiro caso, é possível calcular a integral apresentada em (4.3) analiticamente obtendo-se

$$
\widetilde{\mathcal{N}}_{F}\left(q_{T}, x_{2}\right)=\frac{4 \pi}{Q_{s}^{2}\left(x_{2}\right)} \exp \left(-\frac{q_{T}^{2}}{Q_{s}^{2}\left(x_{2}\right)}\right)
$$

onde a escala de saturação no alvo é dada por $Q_{s}^{A}\left(x_{2}\right)=A^{1 / 3} Q_{s}^{p}\left(x_{2}\right)=A^{1 / 3} Q_{0}\left(x_{0} / x_{2}\right)^{\lambda / 2}$.

Embora não seja possível calcular exatamente a integral que figura em (4.3) no caso do modelo BUW, é possível obter que $\widetilde{\mathcal{N}}_{F}\left(q_{T}, x_{2}\right) \propto 1 / q_{T}^{4}$ para grandes valores de $q_{T}$ [205]. Este é exatamente o comportamento qualitativo esperado para a distribuição não integrada de gluons no limite de grandes valores de momento transversal, quando os efeitos de saturação deixam de ser importantes (regime linear da QCD) e a pQCD padrão, baseada nas equações DGLAP, funciona cada vez melhor com o aumento de $q_{T}$.

A distribuição dos quarks de valência em um núcleo $A, x_{1} q_{v}^{A}\left(x_{1}, Q^{2}\right)$, é obtida através da distribuição de quarks de valência no próton, $x_{1} q_{v}^{p}\left(x_{1}, Q^{2}\right)$, por meio da relação $x q_{v}^{A}\left(x_{1}, Q^{2}\right)=N_{\text {part }} x_{1} q_{v}^{p}\left(x_{1}, Q^{2}\right)$, onde $N_{\text {part }}$ representa o número de nucleons participantes em uma colisão hadrônica $\left(N_{\text {part }}=1\right.$ para colisões $\left.p p\right)$. Nos resultados apresentados a seguir, assumimos que $x_{1} q_{v}^{p}\left(x_{1}, Q^{2}\right)$ é sempre descrita pela distribuição de pártons MRST01LO [105], que também foi utilizada previamente nas referências $[298,301]$. Tal escolha foi simplesmente assumida de modo a possibilitar a comparação direta dos nossos resultados com aqueles obtidos nessas referências.

A Figura 4.1 mostra a comparação de nossos resultados para a distribuição de rapidez da quantidade líquida de bárions, $d N / d y$, em colisões centrais $P b P b$ no SPS $(\sqrt{s}=17.3 \mathrm{GeV})$ e $A u A u$ no RHIC $(\sqrt{s}=64,2$ e $200 \mathrm{GeV})$ com os resultados apresentados nas referências $[298,301]$. De acordo com a abordagem que aqui adotamos, a distribuição de rapidez da produção líquida de bárions é obtida integrando-se a equação $(4.5)$ em $p_{T}\left(0 \leqslant p_{T} \leqslant \sqrt{s} e^{-y}\right.$, onde o limite superior leva em consideração a condição cinemática $\left.x_{F}<1\right)$.

Em todos os painéis que ela apresenta, a curva rotulada por "MT" representa os 
resultados obtidos nas referências [298, 301], onde a distribuição não integrada de gluons é dada pelo modelo GBW (equação (4.9)) e a função de fragmentação pela parametrização fenomenológica [310] $D_{p-\bar{p}}(z)=N z^{a}(1-z)^{b}$, com $N=520142$, $a=11.6, b=6.74$. Conforme discutido nessas referências, este modelo descreve

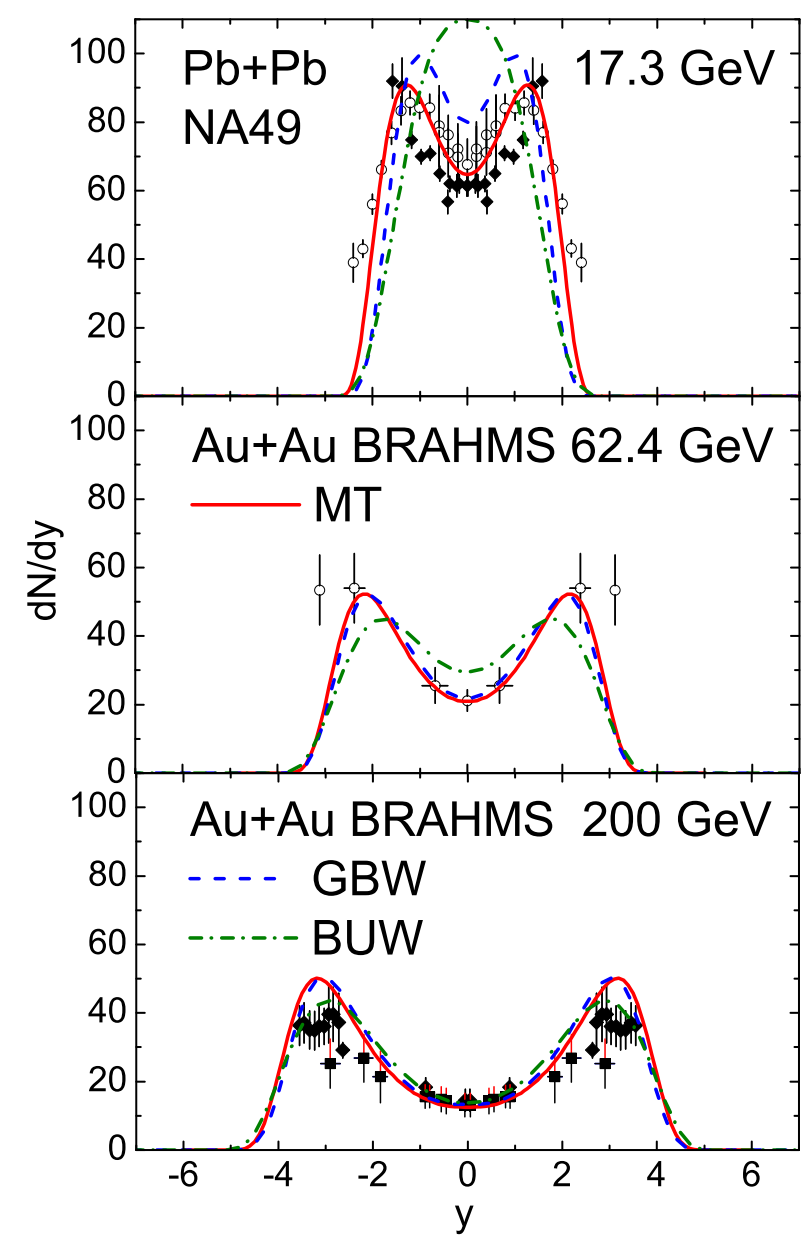

Figura 4.1: Distribuição de rapidez da quantidade líquida de bárions produzida em colisões centrais $P b P b$ no SPS e $A u A u$ no RHIC. Os dados experimentais são das referências [302-306]

razoavelmente bem os dados experimentais para a distribuição de rapidez da produção líquida de bárions nas energias $\sqrt{s}=17,3$ e $64,2 \mathrm{GeV}$, porém superestima os dados para $\sqrt{s}=200 \mathrm{GeV}$ em grandes rapidez.

As curvas rotuladas como "GBW" nesta figura representam os resultados por nós obtidos com a substituição da função de fragmentação fenomenológica acima pela 
função de fragmentação KKP [311]. Apesar do refinamento adotado, é possível observar nessas figuras que a inclusão de um modelo mais realístico ${ }^{3}$ para a fragmentação dos pártons em hádrons implica na não descrição dos dados experimentais na região frontal de rapidez, porém em energias mais baixas. Por outro lado, chama a atenção, os resultados obtidos com MT e GBW são bastante semelhantes a energias mais altas.

Finalmente, as curvas identificadas como "BUW" representam nossos resultados com uso da distribuição de gluons não integrada obtida através do modelo de dipolo BUW (equação (4.8)) que assim como nos resultados apresentados com o modelo GBW, empregamos as parametrizações MRST01LO e KKP para as distribuições de quarks de valência no projétil e para as funções de fragmentação, respectivamente. Como é possível observar, a utilização desses ingredientes melhora a descrição dos dados experimentais na região frontal de rapidez para $\sqrt{s}=200 \mathrm{GeV}$, embora sejam insuficientes para descrever os dados experimentais em energias mais baixas, indicando uma possível limitação desta abordagem nesta situação.

Na Figura 4.2 (a) apresentamos nossas predições com o modelo BUW para as distribuições de rapidez da quantidade líquida de bárions nas energias do LHC. Para efeito de comparação, apresentamos também nossos resultados com os modelos "MT" e "GBW". Como pode ser observado, as três predições são semelhantes ${ }^{4}$. Por completeza, a Figura 4.2 (b) apresenta as previsões para essas mesmas quantidades, porém em colisões minimum bias $p P b$, também nas energias do LHC.

A Figura 4.3 apresenta os resultados para a distribuição de momento transversal da produção líquida de bárions em colisões centrais $A u A u$ no RHIC, obtidos a partir da equação (4.5), para diferentes valores de rapidez. Como na referência [301] assumimos $N_{\text {part }}=315$ e 357 para $\sqrt{s}=62,4$ e $200 \mathrm{GeV}$, respectivamente.

\footnotetext{
${ }^{3}$ Enquanto que a distribuição $D_{p-\bar{p}}(z)$ empregada pelos autores dessas referências resultam de um estudo meramente fenomenológico, a distribuição KKP por nós empregada é oriunda de uma análise global de dados experimentais, de maneira semelhante ao processo utilizado na determinação das PDFs.

${ }^{4}$ Devido à semelhança entre os resultados "MT"e "GBW", onde a função de fragmentação foi o único ingrediente alterado, apresentaremos adiante apenas os resultados obtidos com a função de fragmentação KKP em combinação com cada um dos modelos de dipolo considerados.
} 


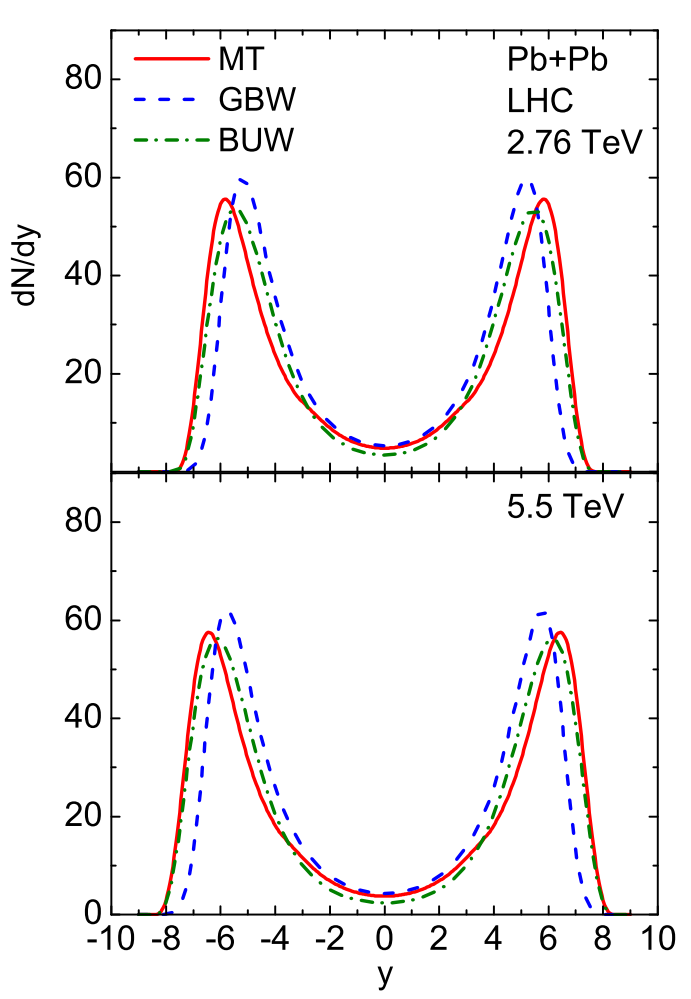

(a)

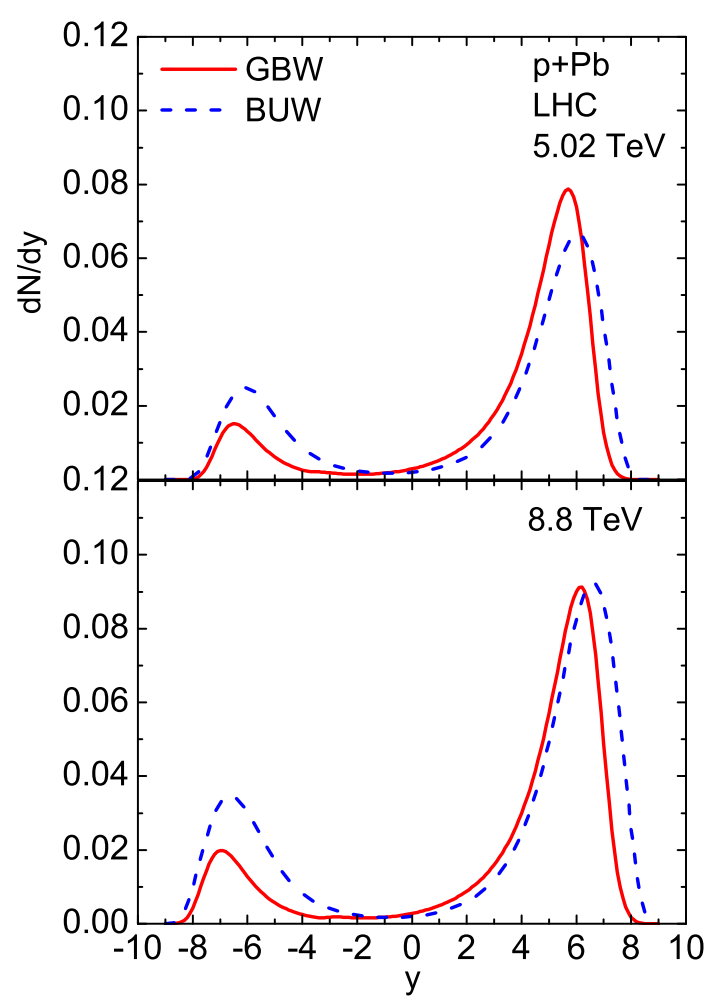

(b)

Figura 4.2: Distribuição de rapidez para a quantidade líquida de bárions produzida em (a) colisões centrais $P b P b$ e (b) colisões minimum bias $p P b$ nas energias do $\mathrm{LHC}$.

É importante notar que os resultados obtidos mostram duas características interessantes. Em primeiro lugar, observa-se um bom acordo entre os dados experimentais quando o espectro de momento transversal é obtido utilizando-se o modelo BUW e, ao mesmo tempo, um desacordo, para $p_{T}>1 \mathrm{GeV}$, quando esta mesma quantidade é obtida considerando-se o modelo GBW. Isto ocorre por conta da amplitude de dipolo no modelo GBW não conter nenhuma informação advinda das equações DGLAP no regime de grande valores de $Q^{2}$ e, portanto, não devendo ser capaz de reproduzir os dados experimentais, como é o caso. Por outro lado, o modelo BUW é capaz de descrever os dados experimentais, já que produz o comportamento correto (com relação aos dados experimentais) no limite de altos valores de $p_{T}$, conforme mencionado anteriormente nesta seção. 

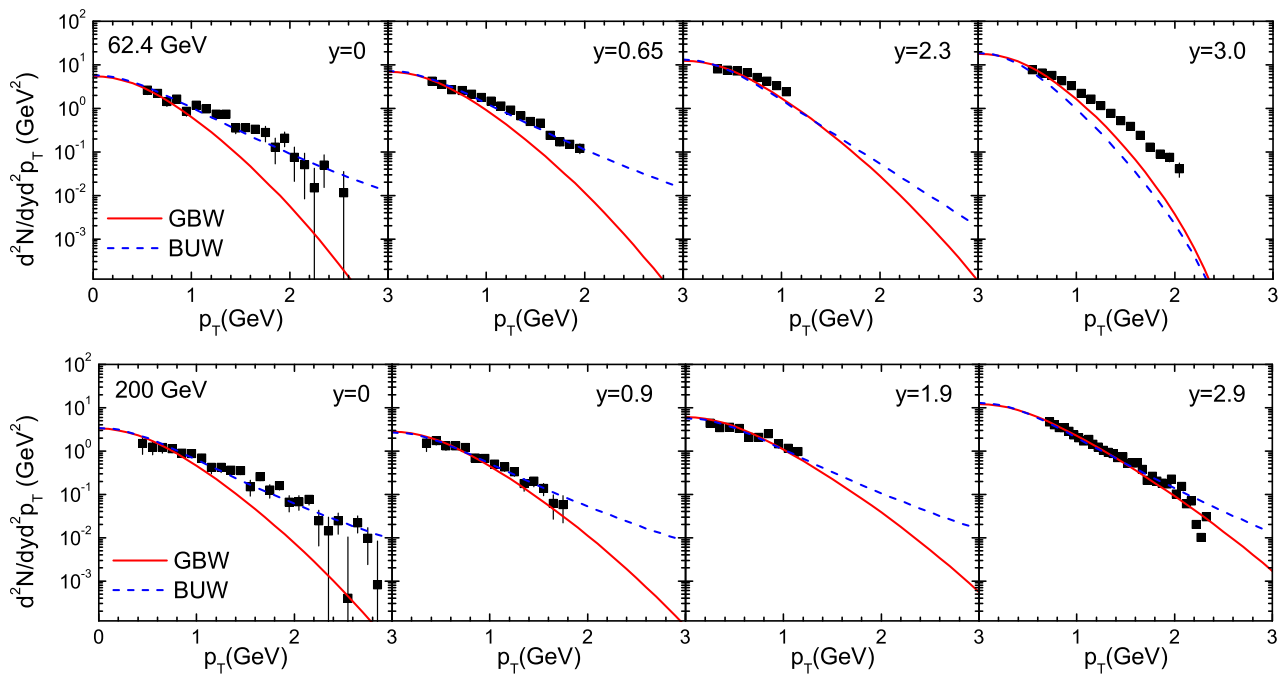

Figura 4.3: Distribuição de momento transversal da produção líquida de bárions em colisões centrais $A u A u$ nas energias do RHIC para diferentes valores de rapidez. Os dados experimentais são das referências [303-306].

A segunda característica importante, que também pode ser notada na Figura 4.3, é a falha do formalismo para maiores valores de rapidez na energia mais baixa considerada. Este fato pode ser uma indicação de que, na região mencionada, os bárions não são produzidos via fragmentação de quarks independentes, que é a física assumida na distribuição KKP. Isso significa que, mais provavelmente, os bárions sejam formados através do processo de coalescência, isto é, a recombinação dos quarks de valência incidentes que emergem, próximos uns aos outros, como partículas dominantes após atravessarem o núcleo alvo.

Na Figura 4.4 apresentamos as previsões para a distribuição de momento transversal de píons (curvas superiores) e prótons (curvas inferiores) produzidos colisões $\mathrm{PbPb}$ em $\sqrt{s}=2.76 \mathrm{TeV}$, considerando-se diferentes valores de rapidez.

Seguindo a tendência já observada nas energias do RHIC, as predições dos modelos BUW e GBW são bastante distintas na região de grandes momentos transversais, em especial na região $y<5$. Para maiores valores de rapidez ambas as predições coincidem, o que está diretamente associado à limitação do espaço de fase disponível para a energia considerada. 


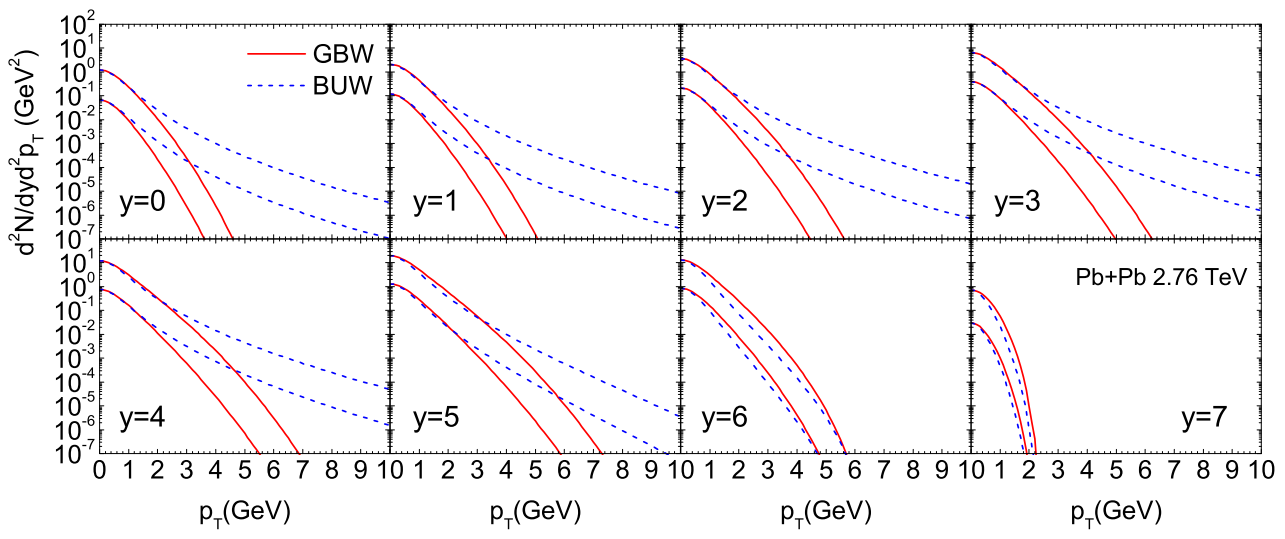

Figura 4.4: Distribuição de momento transversal de pions (curvas superiores) e prótons (curvas inferiores) produzidos em colisões $\mathrm{PbPb}$ a $\sqrt{s}=2.76 \mathrm{TeV}$ e diferentes valores de rapidez.

Visando quantificar a magnitude dos efeitos nucleares na produção líquida de prótons, calculamos o fator de modificação nuclear em colisões próton-núcleo, $R_{p A}$, definido como a razão entre as seções de choque de produção da quantidade líquida de prótons em colisões $p A$ e $p p$, escalonada pelo número de colisões binárias:

$$
R_{p A}=\frac{\frac{d^{2} N_{p A}}{d y d^{2} p_{T}}}{A \frac{d^{2} N_{p p}}{d y d^{2} p_{T}}}
$$

Esse observável físico possuí a seguinte interpretação: quanto mais longe essa quantidade estiver da unidade, mais forte é a indicação de que uma colisão $p A$ não é uma mera superposição de colisões próton-próton independentes, indicando assim a presença de efeitos nucleares. A Figura 4.5 apresenta nossos resultados para essa quantidade considerando-se a produção líquida de prótons utilizando-se o modelo BUW ${ }^{5}$. Como esperado, é possível observar uma supressão da produção da quantidade líquida de prótons na região de pequenos momentos transversais (dentro do regime de saturação) que se intensifica com o aumento da energia. Esta supressão está ligada à presença de efeitos não lineares no (núcleo) alvo.

O formalismo híbrido da teoria efetiva do CGC também pode ser utilizado para

\footnotetext{
${ }^{5} \mathrm{O}$ modelo GBW não foi levado em consideração uma vez que não fornece o comportamento correto da distribuições de momento transversal para grandes valores de $p_{T}$, o que invalida suas predições.
} 


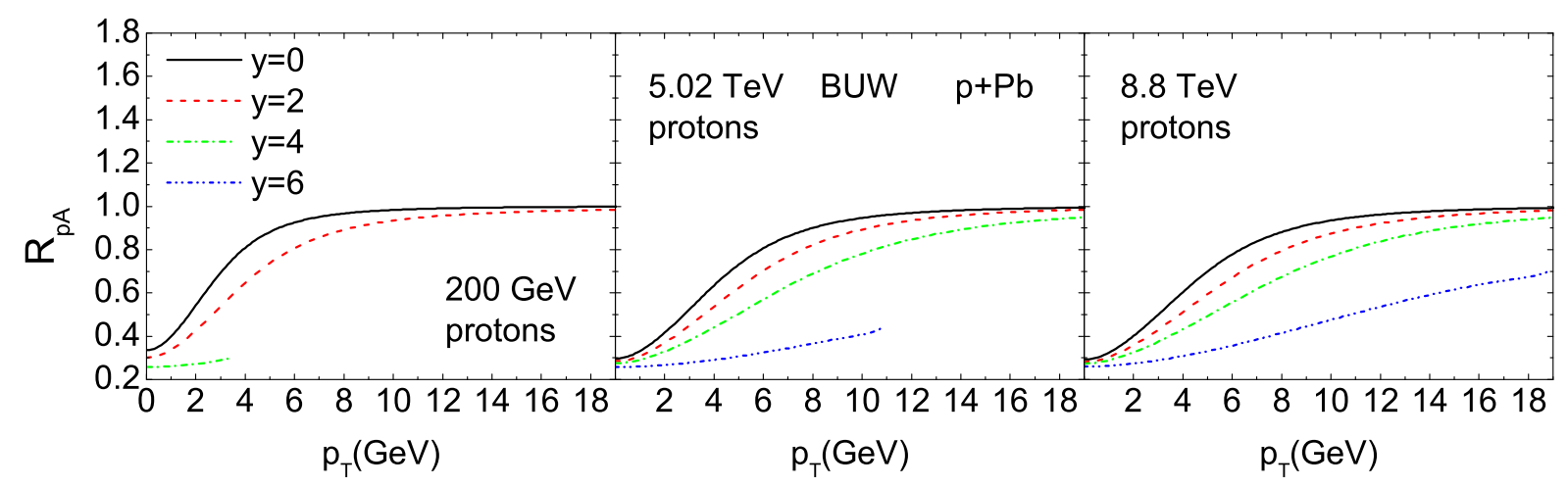

Figura 4.5: Fator de modificação nuclear para a produção líquida de prótons em colisões $p P b, R_{p P b}$ para diferentes valores de energia e rapidez utilizando o modelo BUW.

estudar a diferença com relação à produção da quantidade líquida de prótons e píons na região frontal de rapidez. É oportuno mencionar neste momento que medidas recentes realizadas no LHC [312] confirmaram a existência da chamada "anomalia bariônica", que se traduz no fato da razão entre a produção líquida de prótons e de píons em função do momento transversal ser muito grande (da ordem de 1, a depender do valor de $\left.p_{T}\right)$.

Do ponto de vista teórico, o comportamento exatamente oposto é esperado, uma vez que em uma colisão a altas energias muito mais píons do que prótons deveriam ser produzidos. Esse fato pode ser entendido através de uma simples contagem de partículas elementares: um próton é formado por três quarks de valência, enquanto um méson $\pi$ é formado por apenas um par quark-antiquark o que sugere ser mais "difícil" produzir um próton do que um píon. Outro ponto que corrobora, com essa ideia é o valor de suas massas: a massa de um méson $\pi$ é cerca de 6,7 menor que a massa de um próton, tendo, portanto, um "custo" menor de produção.

A Figura 4.6 mostra os resultados obtidos com o modelo BUW para razão da seção de choque de produção de prótons e píons como função de $p_{T}$. Como pode ser visto a razão $p / \pi$ é pequena, depende fracamente da rapidez, $y$, da energia da colisão e decresce com $p_{T}$. Este é um forte contraste com os dados experimentais, que mostram esta quantidade crescendo com o valor de $p_{T}$ e alcançando valores próximos 


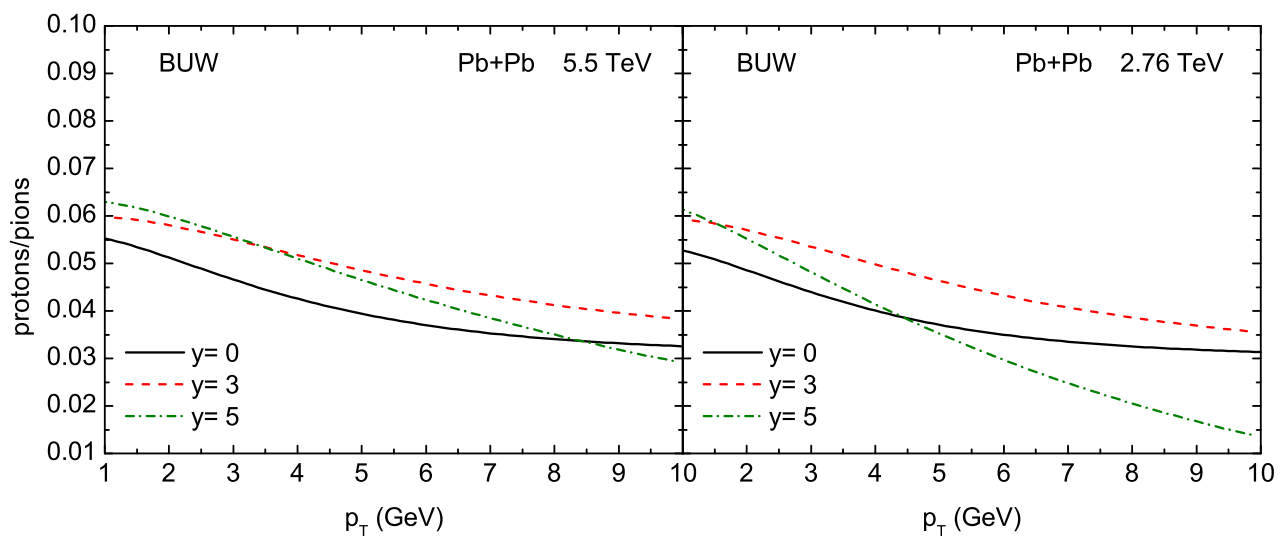

Figura 4.6: Dependência com o momento transversal da razão entre as seções de choque de produção líquida de prótons e píons calculada com o modelo BUW em colisões $P b P b$ para diferentes energias do LHC e diferentes valores de rapidez.

de 1. Consequentemente, conclui-se que no formalismo do CGC não existe anomalia bariônica e píons são sempre mais abundantes que prótons. Isso significa que, segundo a abordagem aqui adotada, essa anomalia deve ter sua origem para prótons e píons produzidos por gluons e quarks do mar na região de rapidez central.

É importante destacar que a produção de bárions na região frontal de rapidez é muito importante para a física de raios cósmicos. Partículas altamente energéticas alcançam o topo da atmosfera terrestre e estão sujeitas a sucessivos espalhamentos a altas energias e em cada uma dessas colisões, um próton projétil, por exemplo, perde energia ao colidir com os íons presentes no meio e cria "chuveiros" de partículas secundárias, eventualmente emergindo como um bárion dominante.

A interpretação de dados experimentais neste tipo de processo depende do conhecimento preciso do espectro de momento dos bárions dominantes e sua dependência com a energia. Uma das questões cruciais a ser resolvida é a existência ou não do chamado "scaling de Feynman", que diz que a distribuição em $x_{F}$ das partículas secundárias produzidas nessas condições é independente da energia.

Eventos envolvendo raios cósmicos provam essencialmente a região de grandes valores de $x_{F}$ do projétil, a região de fragmentação e, portanto, pode-se utiliar o formalismo híbrido da teoria efetiva do CGC no estudo da existência ou não do scaling de Feyn- 
man. Uma motivação adicional para este estudo é que a existência desse scaling será investigada experimentalmente no LHC pela colaboração LHCf $[313,314]$.

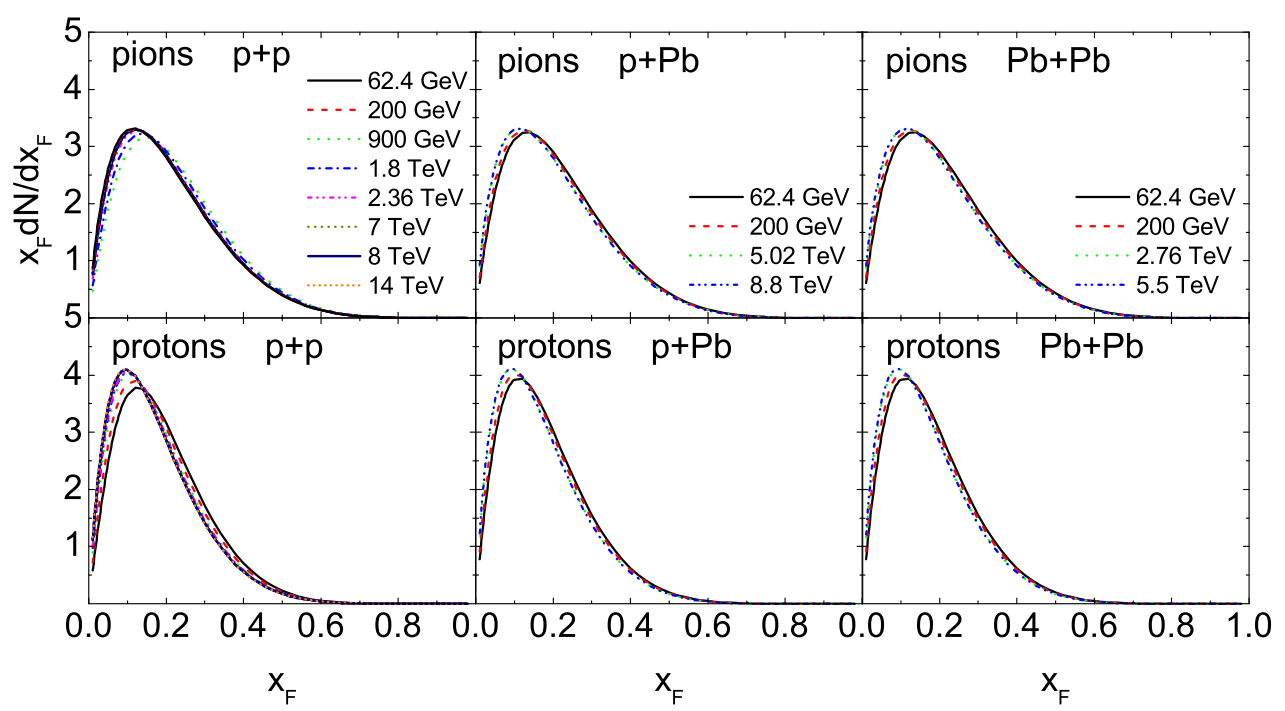

Figura 4.7: Distribuição de $x$-Feynman para píons e prótons dominantes produzidos em colisões $p p, p A$ e $P b P b$ considerando-se diversas energias de colisão.

O espectro em $x_{F}$ de partículas dominantes no formalismo híbrido é obtido simplesmente integrando-se a equação (4.5) em $p_{T}$ e efetuando-se a mudança de variável $y \rightarrow x_{F}$, para a qual $d y=d x_{F} / x_{F}$. A Figura 4.7 mostra nossos resultados para as distribuições em $x$-Feynman para prótons e píons dominantes, produzidos em colisões $p p$, $p P b$ e $\mathrm{PbPb}$ em diversas energias de colisão. Em cada painel pode-se ver claramente um desvio para pequenos valores de $x_{F}$ com o aumento da energia, o que indica um "amolecimento" do espectro de partícula dominante e, portanto, a violação do scaling de Feynman nessas colisões. Esses resultados são compatíveis com aqueles encontrados em [315,316], onde diferentes mecanismos são responsáveis pela sua geração.

A seguir, apresentamos um estudo sobre a produção de mésons $D$ na região de grandes valores de rapidez e de $x_{F}$ em colisões $p p$ onde estimamos a influência da existência do chamado "charme intrínseco" na função de onda do projétil nas distribuições em rapidez e em $x_{F}$ nas energias de interesse para a física de aceleradores e de raios cósmicos. 


\subsection{Produção de mésons $D$ em colisões $p p$ : esti- mando a contribuição do charme intrínseco}

Diferentemente da seção anterior, onde estudou-se a produção de partículas leves esta seção é voltada para a produção de partículas pesadas, mais especificamente mésons.

Partículas leves, tais como os prótons e píons, são compostos por quarks leves: o próton é composto por dois quarks $u$ e um quark $d$ e os mésons $\pi\left(\pi^{+}, \pi^{0}, \pi^{-}\right)$ são compostos por pares quark-antiquark $(u \bar{d}, u \bar{u}$ ou $d \bar{d}, d \bar{u}$, respectivamente). Por outro lado, os mésons pesados possuem ao menos um quark (ou antiquark) pesado, isto é quarks (antiquarks) $c$ ou $b(\bar{c}$ ou $\bar{b})$, em sua composição. Outra característica dos mésons (ou quaisquer outros sistemas) compostos por quarks pesados é o valor da sua massa, $m_{H M} \gtrsim 1 \mathrm{GeV}$, bem maior que a escala de momento do regime não perturbativo da $\mathrm{QCD}, \Lambda_{Q C D}$. Por conta disso, as interações que ocorrem na escala $m_{H M}$ são naturalmente perturbativas.

A produção de estados pesados, tais como mésons $D$ que contém um um quark (antiquark) $c(\bar{c})$ em sua composição, em colisões $p p, p A$ e $A A$ é uma parte importante do programa do LHC e também de futuros colisores [317]. Uma das razões para se estudar a produção de quarks pesados é que se espera que tais partículas sejam sensíveis aos efeitos não lineares da QCD [318-325] e, conforme discutido na seção 4.1, estes realçados na região frontal de rapidez.

Outro motivo vem do fato de que o entendimento da produção de mésons pesados é fundamental para o entendimento do fluxo dos chamados "neutrinos atmosféricos" total a altissímas energias, $E \gtrsim 1 \mathrm{PeV}$ (no referencial do laboratório) [326-329]. Esses neutrinos se originam do decaimento dos hádrons produzidos na interação de partículas altamente energéticas que entram na atmosféra terrestre, os raios cósmicos. Existem duas componentes diferentes para o fluxo de neutrinos atmosféricos: a dos chamados neutrinos "convencionais", que vem do decaimento de mésons carregados leves, como 
píons e kaons, e a componente "direta", onde os neutrinos são produzidos diretamente do decaimento de um méson pesado, tipicamente contendo um quark chame.

Enquanto que o fluxo de neutrinos convencionais diminui rapidamente com a energia [330], uma vez que mésons leves perdem energia ao interagirem com outras partículas antes de poderem decair [327], o mesmo não ocorre com a componente direta, uma vez que os mésons pesados decaem em neutrinos quase que instantaneamente, mesmo a altissímas energias, devido ao seu curto tempo de vida. Como resultado, o fluxo de neutrinos produzidos diretamente do decaimento de mésons pesados domina o fluxo total de neutrinos atmosféricos a altissímas energias. Por fim, a produção direta de neutrinos em processos envolvendo raios cósmicos é um processo que atrapalha em muito [331-333] o estudo do fluxo dos chamados "neutrinos astrofísicos", que são neutrinos produzidos fora da atmosféra terrestre. É interessante mencionar que o fluxo de tais neutrinos pôde ser medido pela colaboração IceCube [334,335].

Como apontado em [329], por exemplo, a principal contribuição para a produção direta de neutrinos vem da produção de mésons pesados na região extremamente frontal de rapidez, além daquela alcançada pelos detectores do LHC, onde novos efeitos podem estar presentes.

No que se segue investigamos a contribuição de quarks pesados "intrínsecos"na função de onda hadrônica [336,337] das partículas colidentes na produção de mésons pesados na região frontal de rapidez.

Quarks pesados no "mar" do próton podem ser gerados perturbativamente, através do "splitting" de um gluon em um par $Q \bar{Q}$, onde $Q(\bar{Q})$ denota um quark (antiquark) pesado. Os quarks gerados desta forma são usualmente ditos quarks pesados "extrínsecos". Por outro lado, os quarks pesados "intrínsecos" possuem múltiplas conexões com os quarks de valência do próton (veja $[336,337]$ para uma discussão mais completa) e, portanto, são de origem não perturbativa.

A maior parte do conteúdo de charme do mar do próton é de origem extrínseca e vem da evolução da distribuição inicial de gluons através das equações DGLAP [15-18]. Este processo é, portanto, bem entendido via pQCD. A existência de uma compo- 
nente de charme intrínseco (intrinsic charm - IC) foi proposta há muito tempo na referência [338] e desde então outros modelos para esta componente vêm sendo discutidos.

No modelo original [338,339], a criação de um par $c \bar{c}$ foi estudada em detalhes. Foi assumido que a função de onda do nucleon possuía estados de Fock de mais alta ordem, um deles sendo $\mid q q q c \bar{c}>$. A probabilidade de encontrar o nucleon nesta configuração é dado pelo inverso do quadrado da massa invariante do sistema. Por conta da presença da massa dos quarks charme e anticharme, esta probabilidade, em função da fração de momento dos quarks, $P(x)$, é muito "dura" (isto é, é não nula para grandes valores de $x(\gtrsim 0.1))$ em comparação com a mesma distribuição obtida a partir das equações DGLAP, quando o par $c \bar{c}$ é gerado perturbativamente. Este modelo ficou conhecido na literatura como BHPS, com cada letra sendo a inicial do sobrenome de cada um dos autores da referência [338]

Existem outros modelos que se propõem a descrever a componente IC além do BHPS. O modelo de nuvem mesônica (Meson Cloud - MC) possui uma abordagem mais dinâmica: neste modelo o nucleon flutua em um estado intermediário composto por um bárion e um méson, um contendo um quark pesado e o outro um antiquark pesado [340-342], que carregam a maior parte do momento do hádron a que está confinado.

A principal diferença entre os modelos BHPS e MC é que este último prediz uma assimetria na distribuição de momento do charme e anticharme que, por sua vez gera uma assimetria na distribuição de momento de mésons $D^{+}$e $D^{-}$[343], por exemplo. Isto acontece pelo fato de charme e anticharme estarem em diferentes estados hadrônicos ligados: um deles em uma configuração bariônica e o outro numa configuração mesônica.

A componente IC da função de onda do próton foi considerada na análise global de dados feita pela colaboração CTEQ em 2006 [344]. Neste "update" a colaboração CTEQ determinou a forma e a normalização da distribuição de IC, seguindo o mesmo procedimento já utilizado para as demais espécies de pártons. Posterior a isso, diversas 
distribuições para a componente IC encontradas na literatura mostraram-se por essa colaboração compatíveis com os dados globais considerados (maiores detalhes sobre os dados experimentais considerados podem ser encontrados em [344]) .

Além dos modelos BHPS e MC, já mencionados, a colaboração CTEQ testou outro modelo para a componente IC. Este modelo consiste em assumir que numa escala de momento pequena, antes mesmo das equações DGLAP serem válidas, já existe algum conteúdo de charme no próton, possuindo uma distribuição de momento típica dos quarks do mar $(\simeq 1 / \sqrt{x})$ com a normalização a ser fixada através do ajuste de dados experimentais. Por essa razão este modelo para a componente IC no próton ficou conhecido como "tipo mar" (Sea Like - SL).

As distribuições da fração de momento resultantes (calculadas na escala $Q=4 m_{c}$, sendo $m_{c}$ a massa do quark charme) para os quarks charme são apresentadas na Figura 4.8 (curvas inferiores), onde a curva "no IC" representa a predição "padrão" da colaboração CTEQ, isto é, sem considerar a presença de uma componente IC nas condições iniciais necessárias para as equações DGLAP. As demais curvas indicam a mesma quantidade, porém na presença de uma componente IC, que é descrita seguindo cada um dos modelos mencionados nos parágrafos anteriores ${ }^{6}$. Como pode ser observado, os modelos BHPS2 e MC2 predizem um grande aumento desta distribuição na região de grandes valores de $x(\gtrsim 0.1)$. Em contraste, o modelo SL2 prediz um aumento menor nesta mesma região, porém prevê um aumento maior na região de pequenos valores de $x(\lesssim 0.2)$. As distribuições de gluons correspondente a cada um dos casos também é apresentada nesta mesma figura (curvas superiores).

Devido à liberdade na regra de soma da fração de momento carregada pelos gluons, a distribuição de gluons também é modificada pela presença de uma componente IC na função de onda do próton. Em particular, como esperado, os modelos BHPS2 e MC2 sugerem uma supressão na distribuição de gluons na região de grandes valores de $x$.

\footnotetext{
${ }^{6}$ Embora existam limites superiores para a fração de momento carregada pela componente IC [336, 344], não há, no entanto, um valor fixo e bem conhecido para esta quantidade. Sendo assim, a colaboração CTEQ considerou dois diferentes valores para fração de momento carregada pelos quarks da componente IC. O sufixo "2" nessas distribuições indica que considerou-se o segundo caso, onde a fração de momento carregada pelos quarks IC é sempre maior quando comparado ao caso "1".
} 


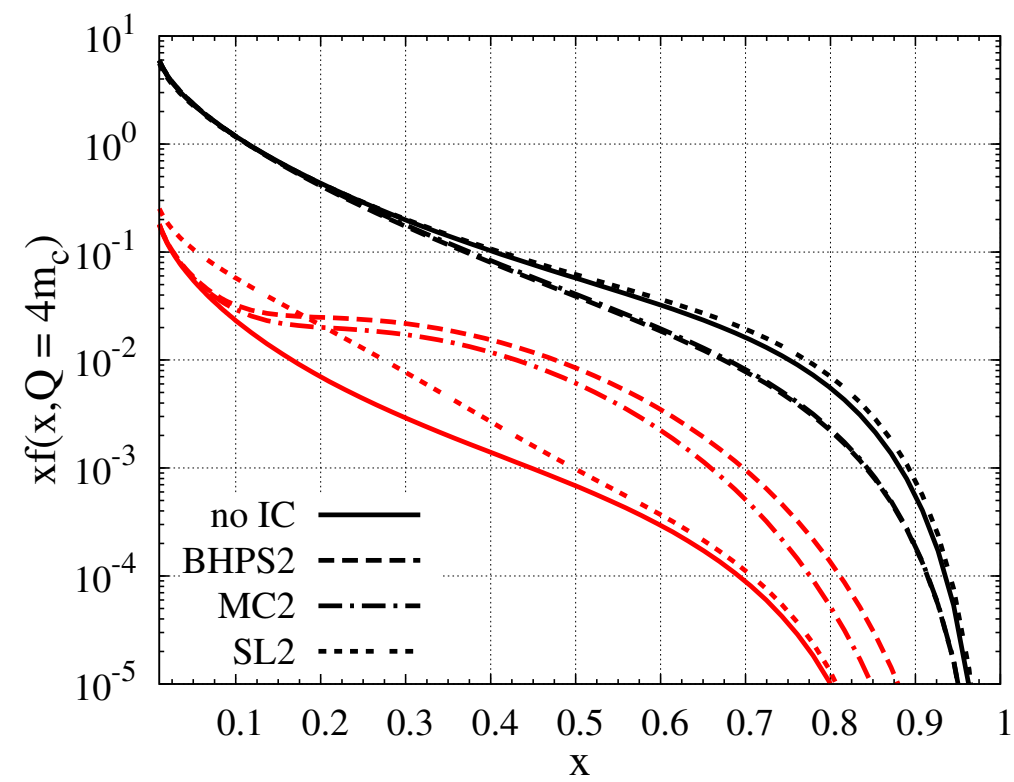

Figura 4.8: Predições para a dependência com a fração de momento $x$ dos quarks charme (curvas inferiores) e dos gluons (curvas superiores) obtidas pela colaboração CTEQ [344] considerando-se (curvas "BHPS2", "MC2"e "SL2") ou não (curva "no IC") a presença de uma componente IC no próton.

Este decréscimo vem diretamente do fato de que a soma total da fração de momento deve ser igual à unidade. Uma discussão mais detalhada pode ser encontrada na referência [345]. Conforme será discutido mais detalhadamente na subseção 4.3.2, esta modificação em $x g\left(x, Q^{2}\right)$ tem implicações importantes na produção de mésons $D$ na região frontal de rapidez.

O aumento da distribuição de quarks charme na região de grandes valores de $x$, que é associado à presença dos (anti)charme intrínsecos, motivou um grande número de estudos fenomenológicos visando confirmar a presença (ou a ausência) dessa componente na função de onda hadrônica. Uma das consequências diretas da existência do charme intrínseco é que ele dá origem a mésons pesados que carregam uma grande fração de momento com relação as demais partículas do feixe colidente. Este fato altera, por exemplo, as distribuições em $x_{F}$ e em $y$ de partículas constituídas por quarks $c$ ou $\bar{c}$. Esta alteração foi explorada anteriormente nas referências [346-348]. Além disso, a presença do charme intrínseco também altera a seção de choque para a produção de 
bósons de Higgs [349] e de fótons [350,351] na região de grandes valores de $x_{F}$.

Novas parametrizações para a distribuição de pártons, levando em consideração a existência de uma componente IC, surgiram nos últimos dois anos [352,353], motivando um intenso debate sobre a quantidade de IC na função de onda do próton $[353,354]$. Ao mesmo tempo, novas implicações foram sendo discutidas em $[355,356]$. A proposta da construção de um experimento com alvos fixos utilizando os mesmos feixes de partículas já utilizados no LHC, para operar a altas energias e fornecer uma grande taxa de eventos (o chamado "AFTER@LHC", veja [357-361] para maiores detalhes), igualmente tem motivado novos estudos teóricos sobre a possibilidade de se medir a componente IC do nucleon [336]. Em particular, e de grande interesse nessa tese, o efeito da componente IC no fluxo de neutrinos atmosféricos medidos pelo IceCube foi estudado nas referências [331-333,362] considerando-se diferentes modelos fenomenológicos para o tratamento da componente intrínseca.

Nesta seção considera-se a produção de mésons $D$ na região frontal de rapidez, ou grandes valores de $x_{F}$, equivalentemente, em colisões hadrônicas. A exemplo da discussão na seção 4.1 e do estudo apresentado na seção anterior, nessas condições a cinemática da colisão é altamente assimétrica e o sistema de colisão projétil-alvo é descrito como um sistema diluído-denso. Os efeitos não lineares da QCD devido à formação de um meio altamente denso no alvo devem, portanto, ser levados em consideração, o que aqui é feito através do uso da teoria efetiva do CGC.

A descrição satisfatória dos dados experimentais nesta região cinemática pela teoria efetiva do CGC $[80,204,205]$ indica que esta abordagem é a ferramenta apropriada para estudar a produção de partículas na região de grandes valores de rapidez. Nesta mesma linha, em [348] o formalismo proposto em [73,204] para a produção de mésons leves foi generalizado para a produção de mésons $D$ em colisões $p p$ e $p A$ na região frontal de rapidez considerando-se a presença de IC na função de onda do projétil ${ }^{7}$.

Em [348] foram apresentadas predições para a distribuição de momento transversal

\footnotetext{
${ }^{7}$ Recentemente a principal equação proposta em [348], equação (1) deste trabalho, foi reobtida em [325].
} 
de mésons $D$ na região frontal de rapidez em colisões $p p$ e $p A$ nas energias do RHIC e do LHC. Os resultados obtidos indicaram que a presença de uma componente IC modifica fortemente este observável. Todavia, a produção de quarks charme extrínsecos, que ocorre via o processo perturbativo $g+g \rightarrow c+\bar{c}$, já mencionado anteriormente, não foi incluída de uma maneira sistemática; de fato esta contribuição foi considerada como um processo de fundo e foi estimada utilizando-se o gerador de eventos PYTHIA.

Um dos objetivos do estudo apresentado nesta seção é a inclusão sistemática desta contribuição, levando-se em consideração os efeitos não lineares da QCD na região de pequenos valores de fração de momento, bem como as modificações na distribuição de gluons que ocorrem na região de grande fração de momento preditas pelos diferentes modelos da componente IC.

Na próxima subseção apresenta-se uma breve revisão das abordagens utilizadas no cálculo da produção de mésons $D$ na região de grandes valores de rapidez e $x_{F}$. Em particular, revisa-se a abordagem proposta em [348] para a contribuição da produção de tais mésons via componente IC e a produção de quarks pesados via interações gluônicas, desenvolvida nas referências [363-365] e posteriormente aplicada em estudos fenomenológicos nas energias do LHC na referência [323]. Na subseção 4.3.2 apresentam-se predições para a seção de choque total de produção de quarks charme em colisões $p p$ nas energias do LHC, bem como a comparação com os dados experimentais já existentes. Resultados para a distribuição de rapidez de mésons $D$, onde estima-se a região onde a componente IC é mais importante, também serão apresentados. Por fim, estima-se a distribuição em $x_{F}$ de quarks charme em colisões $p p$ nas energias do LHC e também de raios cósmicos, $\sqrt{s} \sim 200 \mathrm{TeV}$, onde demonstra-se que esta distribuição é fortemente alterada na região $x_{F} \geq 0.2$ quando da presença de uma componente IC na função de onda do projétili ${ }^{8}$.

\footnotetext{
${ }^{8} \mathrm{~A}$ motivação para esta análise vem do fato de que a distribuição em $x_{F}$ de quarks pesados, especialmente $c$ e $\bar{c}$, é o principal ingrediente no cálculo do fluxo de neutrinos diretos.
} 


\subsubsection{Produção de mésons $D$ na região frontal de rapidez}

Nesta seção trata-se da produção direta de mésons $D$, isto é, desconsiderando-se a contribuição da produção de tais mésons a partir do decaimento de mésons mais pesados. Neste caso, a produção desses mésons é determinada pela seção de choque de produção de quarks pesados, quarks $c / \bar{c}$ mais especificamente.

A seção de choque de produção de quarks pesados pode ser obtida utilizando-se a fatorização colinear da QCD. Neste caso, a produção dessas partículas é descrita em termos de dois processos partônicos básicos: a fusão de gluons, $g+g \rightarrow c+\bar{c}$ e a fusão de um par quark-antiquark, $q+\bar{q} \rightarrow c+\bar{c}$, (embora este último processo seja desprezível a altas energias). A seção de choque elementar desses processos é então convoluída com a PDF e a função de fragmentação correspondente.

Todavia, na região de pequenos valores de fração de momento, $x$, a fatorização colinear deve ser generalizada para ressomar as potências de $\alpha_{s} \ln \left(s / q_{T}^{2}\right)$, onde $q_{T}$ representa o momento transversal do estado final, $\sqrt{s}$ representa a energia do centro de massa da colisão e $\alpha_{s}$ representa a constante de acoplamento forte. Essa ressoma é, em geral, feita na abordagem da fatorização $k_{T}$. Porém, uma vez que nosso interesse é a produção de mésons $D$ na região frontal de rapidez, essa abordagem falha em capturar os graus de liberdade que contém altos valores de $x$ no projétil.

Uma maneira de se estudar a produção de quarks pesados a partir de interações gluon-gluon é considerar o formalismo de dipolo de cor, desenvolvido nas referências [363365], que leva em consideração dois ingredientes chaves: os efeitos não lineares na dinâmica da QCD e correções de mais alta ordem em $\alpha_{s}$ [366]. A ideia básica dessa abordagem é que, antes de interagir com o alvo, um gluon é emitido pelo projétil e flutua em um par $Q \bar{Q}$. O diagrama desse processo é apresentado na Figura 4.9, onde o par $Q \bar{Q}$ foi assumido como um par $c \bar{c}$. Considerando-se que os quarks pesados que formam o dipolo de cor, bem como o gluon incidente (antes de flutuar no par $Q \bar{Q}$ ) podem interagir com o alvo, a distribuição de rapidez, $y$, para uma colisão envolvendo 


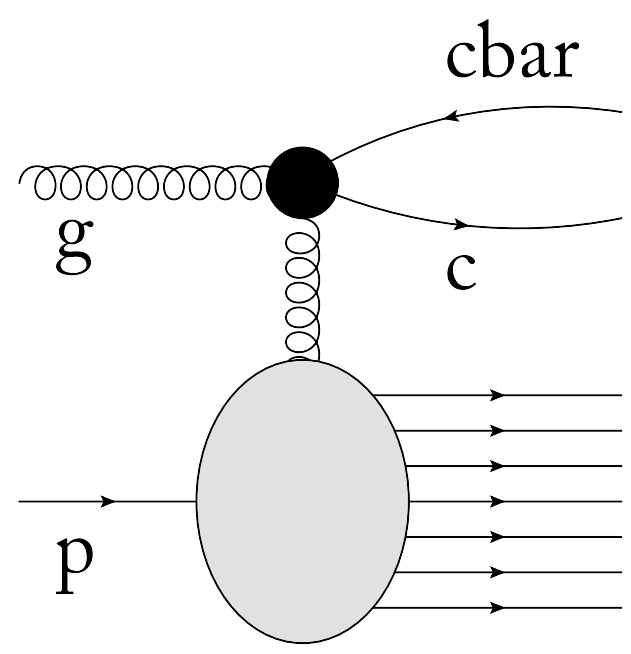

Figura 4.9: Contribuição para a produção de mésons $D$ na região frontal de rapidez a partir de interações gluon-gluon.

dois hádrons, $h_{1} h_{2}$, pode ser escrita da seguinte maneira [365]:

$$
\frac{d \sigma\left(h_{1} h_{2} \rightarrow\{Q \bar{Q}\} X\right)}{d y}=x_{1} g_{h_{1}}\left(x_{1}, \mu_{F}^{2}\right) \sigma\left(g h_{2} \rightarrow\{Q \bar{Q}\} X\right)
$$

onde $x_{1} g_{h_{1}}\left(x_{1}, \mu_{F}\right)$ é a distribuição de gluons no projétil, a seção de choque $\sigma\left(g h_{2} \rightarrow\right.$ $\{Q \bar{Q}\} X)$ descreve a produção do par de quarks pesados em uma interação gluonnucleon, $y$ é a rapidez do par $Q \bar{Q}$ emergente e $\mu_{F}$ é a escala de fatorização utilizada nesses cálculos.

A seção de choque do processo $g+h_{2} \rightarrow Q \bar{Q} X$ é dada por [323,363-365]:

$$
\sigma\left(g h_{2} \rightarrow\{Q \bar{Q}\} X\right)=\int_{0}^{1} d \alpha \int d^{2} \rho\left|\Psi_{g \rightarrow Q \bar{Q}}(\alpha, \rho)\right|^{2} \sigma_{q \bar{q} g}^{h_{2}}(\alpha, \rho)
$$

onde $\alpha(\bar{\alpha} \equiv 1-\alpha)$ representa a fração de momento longitudinal carregada pelo quark (antiquark), $\rho$ representa a separação transversal do par de quarks pesados, $\Psi_{G \rightarrow Q \bar{Q}}$ representa a função de onda (no cone de luz) que descreve a transição $g \rightarrow Q \bar{Q}$

Esta última quantidade pode ser calculada perturbativamente e é dada por

$$
\left|\Psi_{G \rightarrow Q \bar{Q}}(\alpha, \rho)\right|^{2}=\frac{\alpha_{s}\left(\mu_{R}\right)}{(2 \pi)^{2}}\left[m_{Q}^{2} K_{0}^{2}\left(m_{Q} \rho\right)+\left(\alpha^{2}+\bar{\alpha}^{2}\right) m_{Q}^{2} K_{1}^{2}\left(m_{Q} \rho\right)\right]
$$


onde $K_{0}$ e $K_{1}$ representam funções modificadas de Bessel do segundo tipo de ordem 0 e 1 , respectivamente.

Finalmente, $\sigma_{q \bar{q} g}^{h_{2}}$ é a seção de choque de espalhamento do sistema (neutro de cor) quark-antiquark-gluon pelo hádron alvo, $h_{2}$, que é escrita em termos da amplitude de espalhamento dipolo-nucleon, $\sigma_{q \bar{q}}$, da seguinte maneira [363-366]:

$$
\sigma_{q \bar{q} g}^{h_{2}}(\alpha, \rho)=\frac{9}{8}\left[\sigma_{q \bar{q}}(\alpha \rho)+\sigma_{q \bar{q}}(\bar{\alpha} \rho)\right]-\frac{1}{8} \sigma_{q \bar{q}}(\rho)
$$

Como apontado no capítulo 2, a seção de choque dipolo-nucleon pode ser obtida através da amplitude de espalhamento frontal dipolo-nucleon, $\mathcal{N}(\alpha, \rho)$, que carrega toda a informação da dinâmica da QCD,

$$
\sigma_{q \bar{q}}(\alpha, \rho)=\sigma_{0} \mathcal{N}(\alpha, \rho)
$$

onde $\sigma_{0}(=21 \mathrm{mb}$ ) é um parâmetro livre (sua origem esta ligada à integração no parâmetro de impacto, a qual foi desconsiderada neste estudo) que é usualmente fixado através dos dados experimentais do colisor HERA.

De acordo com isso a produção de quarks pesados via formalismo de dipolo de cor é associada às interações gluon-gluon e é determinada pela distribuição de gluons no projétil, assim como pelo modelo assumido para a amplitude de espalhamento dipolonucleon. Obviamente, essa produção depende do valor da massa do quark pesado, um quark charme neste caso, $m_{Q}=m_{c}$, e também da constante de acoplamento forte, $\alpha_{s}$. Por fim, de modo a estimar a seção de choque de produção de mésons $D$, é necessário convoluir a seção de choque de produção de quarks pesados com uma função de fragmentação a ser escolhida.

Como discutido na seção 4.1, o sistema de colisão considerado aqui é do tipo diluído-denso, com os pártons do projétil que carregam grande fração de momento, $x \rightarrow 1$, interagindo com o campo de cor do alvo, que é provado na região de pequenos valores de $x(\rightarrow 0)$. Sendo assim, a seção de choque depende da estrutura partônica 
do projétil na região de grandes frações de momento, $x \gtrsim 0.1$. Caso a componente IC esteja presente na função de onda do projétil, aqui tomado como um próton, ela modifica fortemente a distribuição de pártons exatamente nesta região, e é, portanto, natural esperar que ela também altere a seção de choque de produção de mésons $D$. Ao mesmo tempo, como pequenos valores de $x$ são provados no alvo, os efeitos não lineares da QCD também devem ser considerados. O diagrama de Feynman associado aos mésons $D$ produzidos a partir de uma componente IC no projétil é apresentado na Figura 4.10.

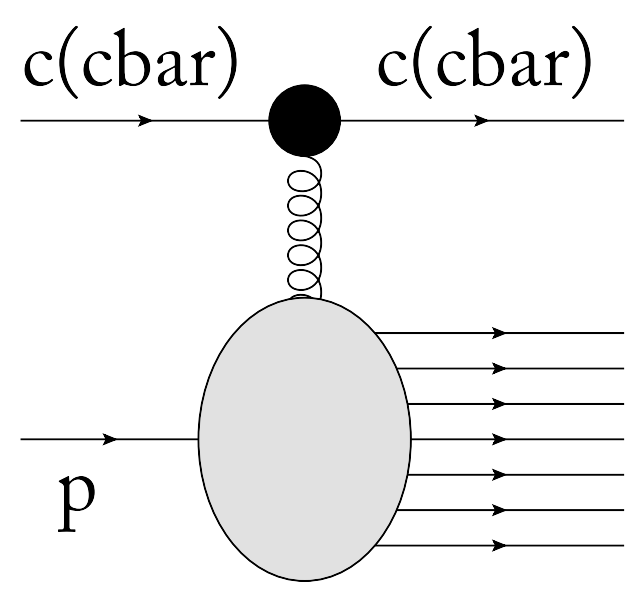

Figura 4.10: Contribuição para a produção de mésons $D$ na região frontal de rapidez a partir de um quark charme no estado inicial. O processo de fragmentação do quark $c / \bar{c}$ em mésons $D$ não é mostrado.

Esse é exatamente o caso onde o formalismo híbrido da teoria efetiva do CGC, discutido em detalhes na seção 4.1, pode ser aplicado. Nesta abordagem, a seção de choque diferencial para a produção de mésons $D$ levando-se em consideração a presença de um quark charme já no estado inicial é dada pelo primeiro termo da equação (4.4) [348],

$$
\frac{d \sigma}{d y d^{2} p_{T}}=\frac{1}{(2 \pi)^{2}} \int_{x_{F}}^{1} \frac{d z}{z^{2}} c_{c / p}\left(x_{1}, q_{T}^{2}\right) \sigma_{0} \tilde{\mathcal{N}}\left(x_{2}, q_{T}\right) D_{D / c}\left(z, \mu^{2}\right)
$$

repetida aqui para o caso de interesse.

Como antes, as variáveis $x_{F}$ e $x_{1,2}$ são definidas por $x_{F}=\sqrt{p_{T}^{2}+m^{2}} e^{y} / \sqrt{s}$ e $x_{1,2}=$ 
$q_{T} e^{ \pm y} / \sqrt{s}$, onde $q_{T}=p_{T} / z$, com $z$ representando a fração de momento do quark charme carregada pelo méson $D$ produzido. Além disso, $c_{c / p}\left(x_{1}, q_{T}^{2}\right)$ representa a distribuição de quarks charme no projétil, $D_{D / c}\left(z, \mu^{2}\right)$ representa a função de fragmentação do charme em um méson $D$ e $\widetilde{\mathcal{N}}\left(x, q_{T}\right)$ representa a transformada de Fourier da amplitude de espalhamento de dipolo na representação fundamental, conforme a equação (4.3).

$\mathrm{Na}$ próxima seção apresentamos resultados para as distribuições de rapidez e $x_{F}$ de mésons $D$ produzidos através das duas contribuições discutidas acima, ou seja, via interações gluon-gluon e a partir de uma componente IC no estado inicial do projétil. De modo a ser consistente, as equações (4.11) e (4.16) são calculadas utilizando-se o mesmo conjunto de PDFs para as distribuições de gluon e de charme, no alvo e os mesmos modelos para a amplitude de espalhamento dipolo-alvo e função de fragmentação já utilizadas anteriormente. Estes resultados encontram-se na referência [79] e estendem a análise da distribuição de momento transversal feita na referência [348], onde a produção de quarks pesados através de interações gluon-gluon não foi considerada.

\subsubsection{Resultados e discussões}

Nesta seção apresentamos predições para a seção de choque total de produção de quarks charme e para as distribuições de rapidez e de $x_{F}$ para a produção de mésons $D$ na região frontal de rapidez e grandes valores de $x_{F}$, considerando-se as duas contribuições discutidas na seção anterior.

Como pode ser observado, ambas as contribuições são escritas em termos da amplitude de espalhamento frontal dipolo - alvo. Na obtenção dos resultados apresentados a seguir considerou-se o modelo BUW [205], apresentado na seção 2.3.4, para esta quantidade. Recentemente, este modelo teve seus parâmetros atualizados em [80] para descrever simultaneamente as distribuições de momento transversal nas energias do RHIC e do LHC em colisões $p p$ e $p A$ e também os dados em colisões ep no HERA ${ }^{9}$.

\footnotetext{
${ }^{9}$ Maiores detalhes sobre o procedimento e os dados experimentais considerados nesta atualização podem ser encontrados na seção 4.4 .
} 
Diferente do estudo apresentado na seção 4.2, em que se considerou-se os valores originais da referência [205], no que se segue utilizamos os parâmetros obtidos em [80]: $a=2, b=125, \gamma_{s}=0,74$ e $\sigma_{0}=21 \mathrm{mb}$.

De modo a quantificar o impacto da componente IC considerando-se o maior número possível de modelos para esta componente, fizemos uso da parametrização CTEQ6.5 [344] para distribuição de pártons no projétil. Esta parametrização possui dois conjuntos diferentes para os modelos da componente IC discutidos na introdução (BHPS, MC e SL), cada um deles considerando um valor diferente para a fração de momento carregada pelo par $c \bar{c}$ no estado inicial do projétil. Como já apontado, aqui são apresentados apenas os resultados obtidos com o conjunto que assume a maior fração de momento carregada pela componente IC para cada modelo considerado (denotados por BHPS2, MC2 e SL2).

É importante mencionar neste momento que a colaboração CTEQ-TEA também realizou uma nova análise global, incluindo dados experimentais mais recentes, não disponíveis na época em que a análise global que deu origem à parametrização CTEQ6.5 foi feita, onde também considerou-se uma componente IC, resultando na distribuição de pártons CT14 [352]. Entretanto, a análise em que a componente IC foi considerada foi realizada em duas ordens seguintes à dominante em $\alpha_{s}$ e não incluiu o modelo MC. Como as equações consideradas neste estudo sobre a produção de mésons $D$ foram derivadas em ordem dominante em $\alpha_{s}$, acreditamos que seria mais consistente utilizar uma distribuição de pártons nesta mesma ordem, que é o caso da distribuição CTEQ6.5. Visando analisar a influência dessa PDF mais recente, porém em ordem dominante, apresentamos em alguns dos nossos resultados as predições obtidas com a PDF CT14 LO, que não considera a existência de uma componente IC.

Nossos resultados mostraram que a seção de choque total da produção de charme e as distribuições de rapidez e $x_{F}$ são observáveis que não são fortemente afetados pela modelagem da função de fragmentação. Por esta razão, assumimos um modelo ingênuo, isto é, sem dinâmica, para a função de fragmentação de um quark charme em um méson $D: D_{h / c}(z)=f_{h} \delta(1-z)$, onde $f_{h}$ é a fração de quarks charme que se 
fragmenta em um hádron $h$. Para o méson $D^{0}$ considerou-se $f_{D^{0}}=0.565$, o mesmo valor utilizado na referência [327]. Além disso, assumimos que a escala de fatorização na equação (4.11) é igual a $\mu_{F}^{2}=4 m_{c}^{2}$ e que $\alpha_{s}=\alpha_{s}\left(\mu_{F}^{2}\right)$. Como consequência, nossos resultados dependeram apenas da escolha do valor da massa do quark charme.

Finalmente, é importante enfatizar que as predições apresentadas aqui com o uso das equações (4.11) e (4.16) podem ser modificadas pela inclusão de correções de mais alta ordem, que, neste e em diversos outros casos, são realizadas de maneira efetiva no valor de um fator multiplicativo constante de modo a tornar possível a descrição dos dados experimentais. Em nossa análise adotamos uma postura mais conservadora e assumimos o valor $K=1$, ou seja, não consideramos correções de mais alta ordem às equações equações (4.11) e (4.16).

A Figura 4.11 (a) mostra a comparação dos resultados obtidos com as escolhas discutidas acima e diferentes valores para a massa do quark charme, para a seção de choque total de produção de charme com os dados experimentais existentes a altas energias. O rótulo "total" indica que estes resultados referem-se à soma das contribuições de quarks charme produzidos via interações gluônicas e diretamente através de um quark charme no estado inicial do projétil. O rótulo "no IC"indica que assumiuse que a distribuição de gluons e de charme no projétil são dadas pela parametrização CTEQ6.5 sem a presença de uma componente IC.

Como os resultados obtidos através da teoria efetiva do CGC não são confiáveis a baixas energias, optamos por não considerar essa comparação na região $\sqrt{s} \lesssim 100$ GeV. No entanto, como pode ser observado, os dados experimentais são descritos razoavelmente bem na região de altas energias para $m_{c}=1,4 \mathrm{GeV}$ e $1,5 \mathrm{GeV}$. Os resultados apresentados a partir da Figura 4.11 (b) foram obtidos com $m_{c}=1,4 \mathrm{GeV}$. No sentido de comparar e também estimar a dependência de nossos resultados com a escolha da escala de fatorização, $\mu_{F}$, apresentamos nessa figura nossas predições com a PDF CT14 LO para diferentes valores de $\mu_{F}$. Considerando-se o intervalo $m_{c}^{2} \leqslant \mu_{F}^{2} \leqslant 16 m_{c}^{2}$, observa-se que a parametrização CT14 LO dá origem à região hachurada apresentada em ambas as figuras, demonstrando que o comportamento a 


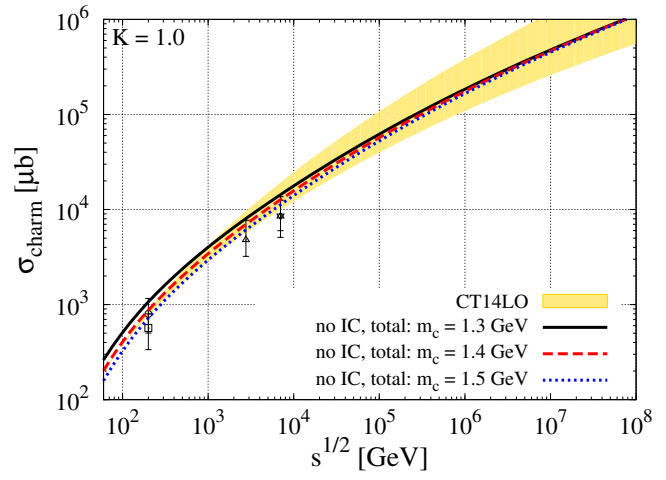

(a)

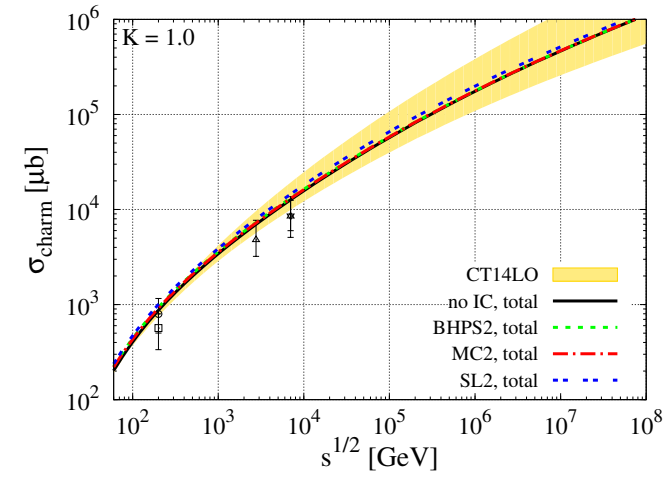

(b)

Figura 4.11: Dependência da seção de choque total para a produção de quarks charme com a energia considerando (a) diferentes valores para a massa do charme e (b) diferentes modelos para a componente intrínseca utilizando-se $m_{c}=1,4$ GeV. Os dados experimentais são das referências [367-372].

altas energias desse observável depende fortemente da escolha para a escala $\mu_{F}$.

Na Figura 4.11 (b) investigamos o impacto dos diferentes modelos para a componente IC na seção de choque total para a produção de charme. É possível perceber que as predições são quase idênticas, o que é esperado, já que este observável é dominado pela produção de charme na região central de rapidez, associada à contribuição gluônica. Como consequência, as modificações associadas à presença de uma componente IC no projétil é negligível para este observável.

A seguir, as predições para a distribuição de rapidez de mésons $D^{0}$ e $\bar{D}^{0}$ produzidos em colisões $p p$ em $\sqrt{s}=7 \mathrm{TeV}$ e $13 \mathrm{TeV}$ são apresentadas nas Figuras 4.12 (a) e (b), respectivamente. Considerando-se os resultados com a distribuição CT14 LO é possível observar que as predições na região central são extremamente sensíveis à escolha de $\mu_{F}$, com as incertezas diminuindo na região de grandes valores de rapidez. As predições com a distribuição CTEQ6.5 sem a inclusão da componente IC e com a inclusão desta componente por meio dos modelos BHPS2 e MC2 predizem um comportamento similar na região de pequenos valores de rapidez, diferindo apenas na região extremamente frontal de rapidez. Por outro lado, o modelo SL2 prediz um aumento na distribuição de rapidez na região de valores intermediários de $y$, que indica um comportamento diretamente associado ao aumento da distribuição de charme para $x \leq 0.2$ neste 


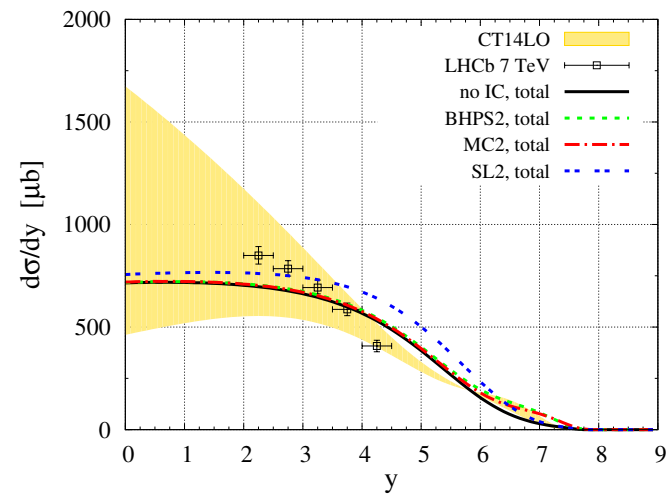

(a)

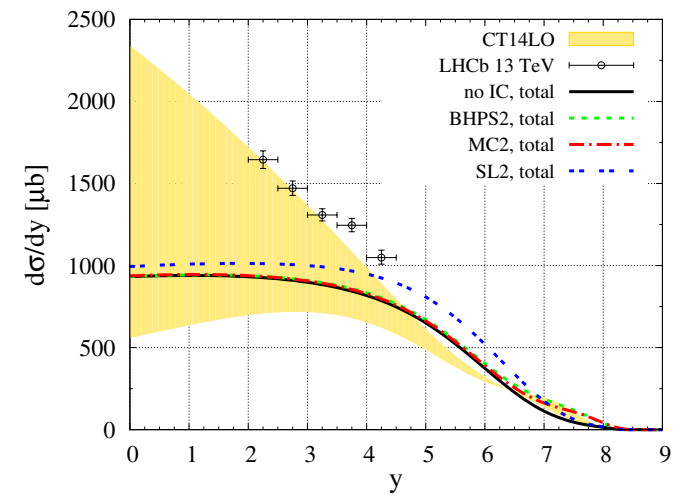

(b)

Figura 4.12: Distribuição de rapidez de mésons $D^{0}+\bar{D}^{0}$ produzidos em colisões pp em (a) $\sqrt{s}=7 \mathrm{TeV}$ e (b) $\sqrt{s}=13 \mathrm{TeV}$. Os dados experimentais são das referências [371, 372].

modelo (vide Figura 4.8).

Com o intuito de quantificar a influência da componente intrínseca e determinar a região cinemática alterada por sua presença, na Figura 4.13 mostramos a dependência com a rapidez da razão entre as predições que consideram a componente intrínseca e a predição da distribuição CTEQ6.5 sem essa componente (denotada por "no IC") nas mesmas energias da figura anterior. O comportamento dessa razão revela que a

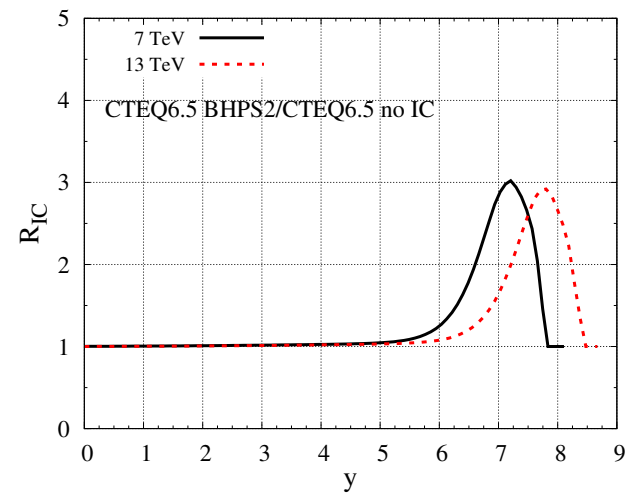

(a)

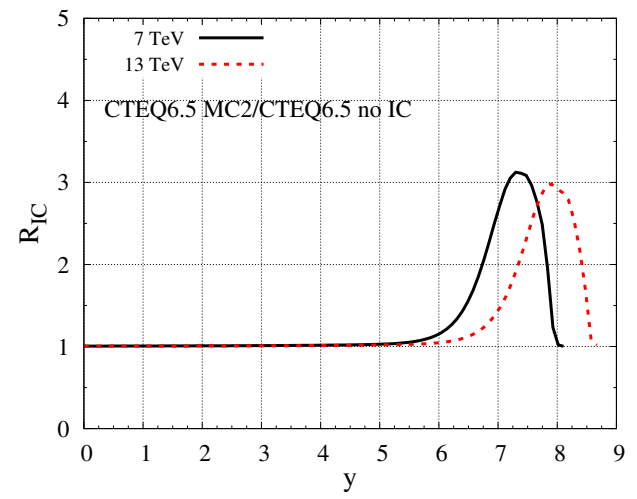

(b)

Figura 4.13: Dependência com a rapidez da razão entre os resultados com e sem a componente IC da parametrização CTEQ6.5 nas energias $\sqrt{s}=7$ e $13 \mathrm{TeV}$ considerando (a) o modelo BHPS e (b) o modelo MC.

componente intrínseca modifica a distribuição de rapidez na região extremamente fron- 
tal, além daquelas alcançadas pela colaboração LHCb, cujo valor máximo alcançado é $y \sim 5$ [373]. Além disso, essa razão chega a ser aumentada por um fator $\approx 3$, com a posição do aumento máximo se deslocando para valores maiores de rapidez a medida que a energia do centro de massa aumenta. Esse comportamento pode ser facilmente entendido lembrando-se que os valores de $x$ provados no projétil são aproximadamente $x \approx\left(m_{T} / \sqrt{s}\right) \exp (+y)$, onde $m_{T}=\sqrt{4 m_{c}^{2}+p_{T}^{2}}$. Consequentemente, o mesmo valor de $x$ no projétil é provado para um valor de rapidez maior a medida que a energia aumenta.

Embora, segundo as predições apresentadas, a componente IC se manifesta em uma região de rapidez além daquela alcançada pelo LHC, essa componente pode trazer implicações em outros observáveis, em particular o fluxo de neutrinos diretos, brevemente discutido na seção 4.3, que é fortemente dependente das características da produção de mésons $D$ na região extremamente frontal. Como assinalado anteriormente [329], a principal contribuição para o fluxo desses neutrinos vem de valores de rapidez além daqueles provados pelos detectores do $\mathrm{LHCb}$, exatamente onde nossos resultados apontam o maior impacto da componente IC.

Um dos principais ingredientes para o cálculo do fluxo de neutrinos a partir do decaimento de mésons contendo quarks charme é a distribuição em $x_{F}$ desses mésons. Sendo assim, é interessante analisar a influência da componente IC nesse observável para diferentes energias. A Figura 4.14 mostra as nossas predições para a distribuição em $x_{F}$ de mésons $D^{0}$ e $\bar{D}^{0}$ produzidos em colisões $p p$ a $\sqrt{s}=13 \mathrm{TeV}$. Nesta figura, as duas contribuições para a produção de tais mésons, isto é, a partir de interações gluônicas ou diretamente pelo quark charme no estado inicial do projétil, são apresentadas separadamente e a curva denotada por "total" representa a soma dessas duas contribuições.

A título de comparação, apresentamos na Figura 4.14 (a) as predições para a distribuição em $x_{F}$ quando a distribuição CTEQ6.5 não considera uma componente IC nas condições iniciais da evolução DGLAP. Neste caso, a distribuição em $x_{F}$ é dominada pela produção de mésons D's a partir de interações gluon-gluon, que é sempre 


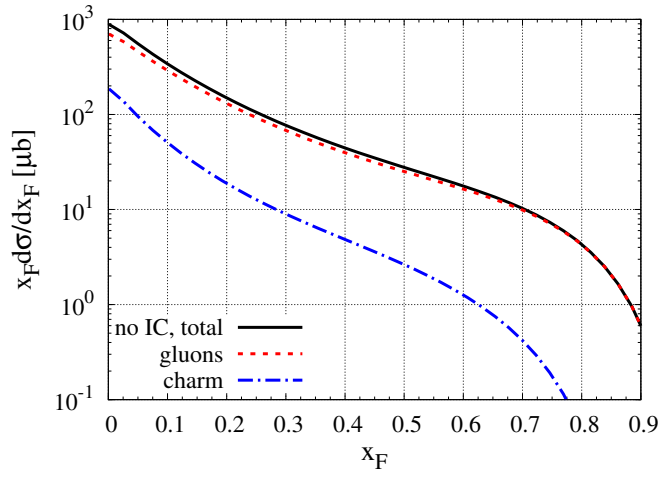

(a)

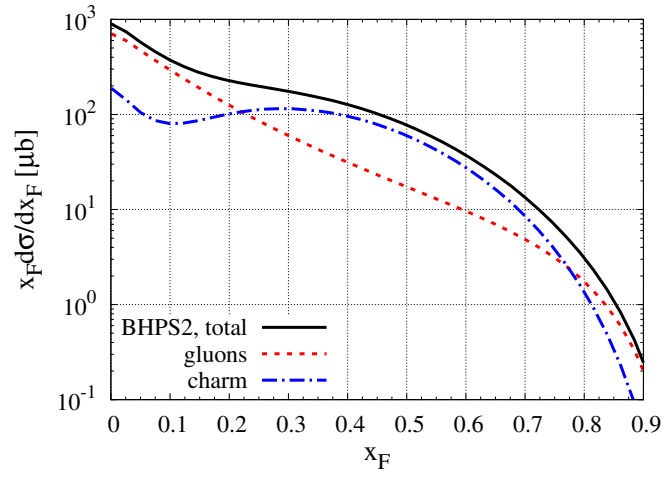

(b)

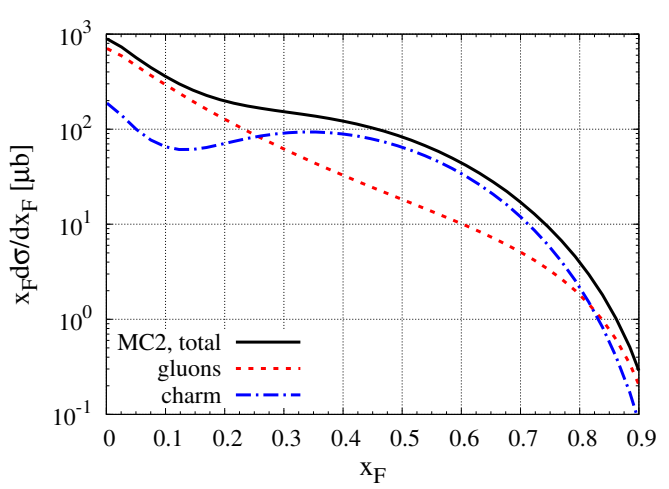

(c)

Figura 4.14: Distribuição de $x$-Feynman de mésons $D^{0}+\bar{D}^{0}$ produzidos em colisões $p p$ em $\sqrt{s}=13 \mathrm{TeV}$ considerando a distribuição CTEQ6.5 (a) sem uma componente IC, (b) com modelo BHPS2 para a componente IC e (c) com modelo MC2 para a componente IC. A contribuição gluônica e de charme são apresentadas separadamente.

maior que a contribuição de mésons de $D$ 's produzidos diretamente a partir de quarks charme. Em contraste, quando a componente IC é considerada, o comportamento dessa distribuição na região de valores intermediários de $x_{F}\left(0.2 \leq x_{F} \leq 0.8\right)$ é fortemente alterado, como pode ser observado nas Figuras 4.14 (b) e (c), onde os modelos BHPS2 e MC2 para a componente IC foram considerados. É importante enfatizar que a contribuição gluônica é maior na região de grandes valores em $x_{F}$ devido ao fato que a distribuição de quarks charme decresce mais rapidamente que a distribuição de gluons na região de grandes valores de $x$, como pode ser visto na Figura 4.8.

De modo a analisar a dependência com a energia da distribuição em $x_{F}$, na Figura 


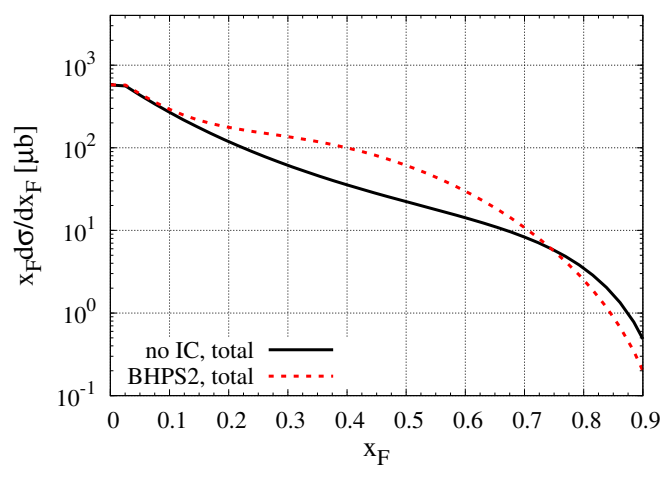

(a)

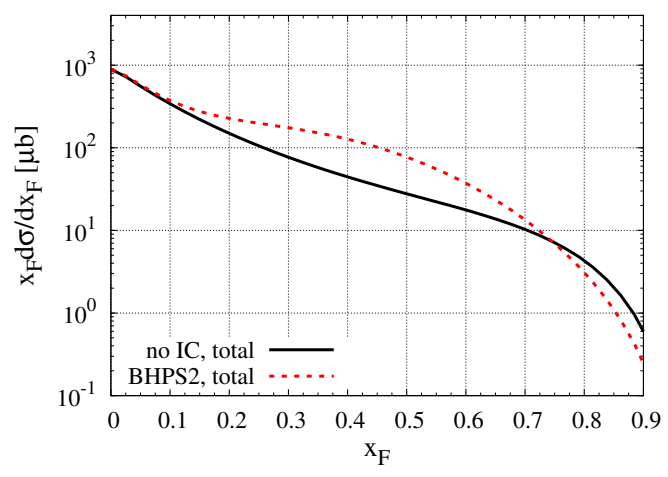

(b)

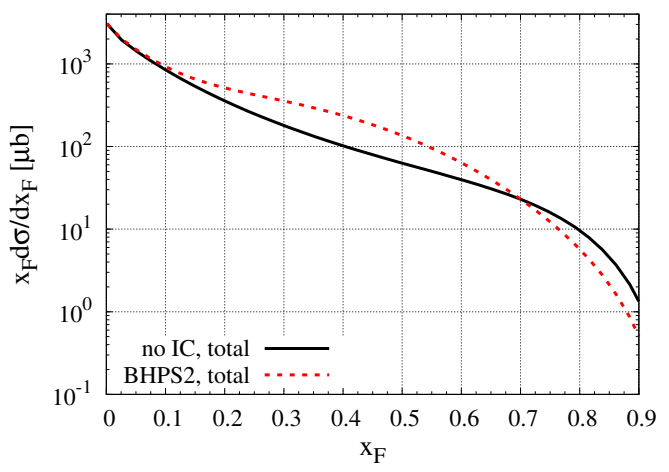

(c)

Figura 4.15: Distribuições de $x$-Feynman de mésons $D^{0}+\bar{D}^{0}$ produzidos em colisões $p p$ em (a) $\sqrt{s}=7 \mathrm{TeV}$, (b) $\sqrt{s}=13 \mathrm{TeV}$ e (c) $\sqrt{s}=200 \mathrm{TeV}$ a distribuição CTEQ6.5 sem a componente IC e com a componente IC descrita pelo modelo BHPS2. Os resultados com o modelo SL2 para a componente IC são similares aos resultados "no IC" $\mathrm{I}$ os resultados com o modelo MC2 para a componente IC são similares ao resultados do modelo BHPS2.

4.15 apresentamos a predição para a distribuição de mésons $D$ 's produzidos em colisões $p p$ em (a) $\sqrt{s}=7 \mathrm{TeV}$, (b) $\sqrt{s}=13 \mathrm{TeV}$ e (c) $\sqrt{s}=200 \mathrm{TeV}$. Este último valor é equivalente à energia provada quando raios cósmicos ultra energéticos interagem com a atmosfera terrestre. Nestas figuras apenas as predições sem a componente IC e com a componente IC descrita pelo modelo BHPS2 são apresentadas. Os resultados com os modelos SL2 e MC2 são semelhantes aos resultados obtidos sem a componente IC e com o modelo BHPS2, respectivamente.

Um aspecto interessante dessa análise é que a predição BHPS2, assim como a predição $\mathrm{MC} 2$, é maior que a predição sem a componente $\mathrm{IC}$, e portanto à predição 


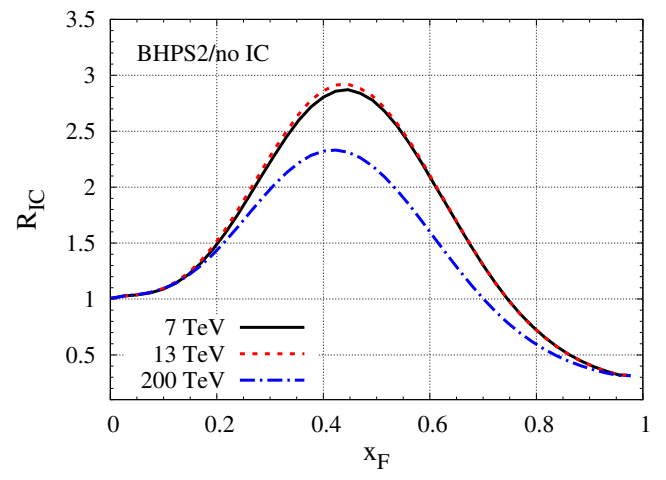

(a)

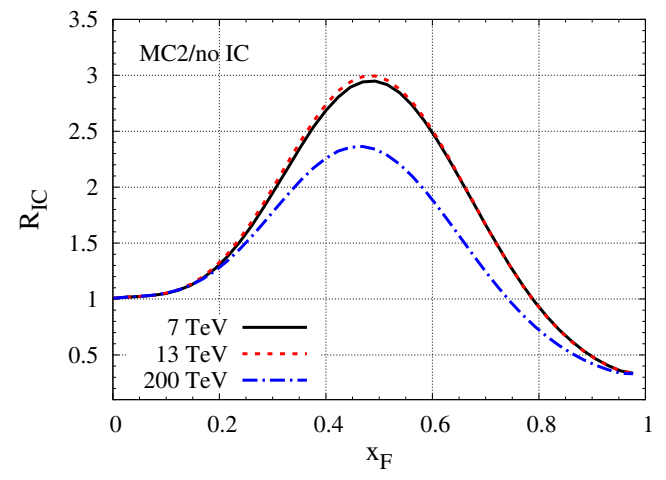

(b)

Figura 4.16: Dependência com $x$-Feynman da razão entre a predição com os modelos para a componente IC e a predição sem a componente IC considerandose (a) o modelo BHPS2 e (b) o modelo MC2 para a componente IC.

SL2, nos valores intermediários de $x_{F}$, mas torna-se menor em valores muito grandes de $x_{F}$. Este comportamento está relacionado a dois fatores: primeiro, o fato de que a contribuição gluônica domina na região de extremos valores de $x_{F}$, como pode ser visto na Figura 4.14 e, segundo, o fato de que a distribuição de gluons na região de grandes valores de $x$ obtida pela colaboração CTEQ6.5 sem a componente IC é maior que aquela que assume sua existência (descrita pelo modelo BHPS2) como pode ser visto na Figura 4.8.

Afim de determinar a magnitude do impacto da componente IC e a região cinemática influenciada pela sua presença, apresentamos nas Figuras 4.16 (a) e (b) o comportamento da razão das distribuições em $x_{F}$ obtidas com os modelos de IC (BHPS2 e MC2) com aquelas onde a componente IC está ausente.

É importante notar que, como consequncia dos resultados da Figura 4.15, os modelos BHPS2 e MC2 predizem um aumento na região de $x_{F}$ intermediário e uma supressão na região de valores muito grandes de $x_{F}$, como esperado. Além disso, o aumento dessa razão é semelhante para ambos os modelos, chegando a um valor 2,2 3 nas energias consideradas.

Finalmente, o principal aspecto dessa análise é o fato de que o aumento dessa razão, devido à presença da componente $\mathrm{IC}$, ocorre exatamente na região de $x_{F}$ onde 
a contribuição de mésons $D$ 's para a produção de neutrinos diretos é dominante [329]. Consequentemente, espera-se que a presença de uma componente IC modifique as previsões para o fluxo de neutrinos diretos produzidos na atmosfera medidos pela colaboração IceCube. Essa verificação, no entanto, está além dos objetivos do estudo apresentado nessa seção.

A seguir, apresentamos um estudo sobre a dependência com a rapidez do momento transversal médio de partículas produzidas em colisões $p p$ e $p P b$, um observável que pode ser medido experimentalmente no LHC.

\subsection{O momento transversal médio de partículas em colisões hadrônicas e sua dependência com a rapidez}

Com o início das atividades do LHC e o fabuloso aumento de energia experimentalmente disponível para as colisões hadrônicas, regiões cinemáticas antes inacessíveis passaram a ser alcançadas tornando possível testar a QCD em novos regimes. Em particular, processos físicos associados à região frontal de rapidez (como, por exemplo ${ }^{10}$, processos difrativos, produção exclusiva de partículas e a dinâmica da região de pequeno valores de $x$ ) têm impulsionado inúmeros estudos, cujas hipóteses, modelos e resultados estão sendo colocados à prova.

Em geral, os processos que ocorrem nessas condições, de altas energias e grandes valores de rapidez, são caracterizados pela produção de partículas com pequeno momento transversal e associados ao regime não perturbativo da QCD, portanto, cujo estudo ainda não pode ser feito a partir de primeiros princípios.

Todavia, nessas condições espera-se a formação do CGC, em cujo cenário a função de onda do projétil em tais processos é provada na região de grandes valores de $x$,

\footnotetext{
${ }^{10}$ Maiores exemplos podem ser encontrados em [374].
} 
podendo ter sua evolução descrita pelas equações de evolução padrão da QCD, as equações DGLAP, enquanto que o alvo é provado na região de pequenos valores de $x$, onde a distribuição de gluons satura devido à inclusão dos processos de recombinação e efeitos coerentes [35-47]. Como discutido na seção 4.1, o meio formado nessas colisões fica caracterizado pelo surgimento de uma nova escala dinâmica, a escala de saturação $Q_{s}$, que passa a controlar os principais ingredientes da produção de partículas, que evoluem segundo uma hierarquia infinita de equações acopladas para as correlações das chamadas "linhas de Wilson" [35-40,44-47].

A altas energias e grandes valores de rapidez, é esperado que $Q_{s}$ se torne muito maior que a escala de confinamento da $\mathrm{QCD}, \Lambda_{Q C D}$, justificando uma abordagem perturbativa para a produção de parículas nesta região. Além disso, espera-se que a escala de saturação determine o momento transversal típico dos pártons produzidos na interação entre as partículas colidentes. Isso implica que a medida do momento transversal médio das partículas produzidas em colisões hadrônicas, $\left\langle p_{T}\right\rangle$, pode fornecer informações importantes sobre a dinâmica da QCD.

Outra importante motivação para a uma análise detalhada de $\left\langle p_{T}\right\rangle$ em colisões $p p$ e $p A$ é a recente sugestão feita na referência [375] de que esta quantidade poderia ser utilizada para separar as predições da hidrodinâmica e do CGC para o efeito ridge (discutido na seção 2.2.3) observado em colisões $p p$ e $p(d) A$ de alta multiplicidade $[151,153,179,181,184,185,376,377]$. Enquanto esse tipo de estrutura (ridge) observada em colisões de íons pesados $(A A)$ no RHIC e no LHC é tida como uma evidência da natureza hidrodinâmica do QGP [378,379] ainda não existe uma razão convincente para que esses sistemas de colisão menores também exibam um comportamento hidrodinâmico, embora modelos que se valem desse tipo de abordagem sejam capazes de descrever dados experimentais oriundos desses sistemas [142, 143, 146, 380].

A razão por trás disso é o fato de as equações hidrodinâmicas são derivadas assumindo-se uma clara separação entre as escalas de comprimento macroscópicas (o tamanho do sistema colidente, por exemplo) e microscópicas (regiões densas com comprimento da ordem de $1 / Q_{s}$ presentes nos núcleos colidentes, por exemplo). Nos 
casos onde essa separação é clara, o sistema comporta-se como um fluído e tem seu comportamento governado pelas equações da hidrodinâmica relativística. Entretanto, no caso onde a separação dos graus de liberdade microscópicos e macroscópicos não é clara, o que ocorre quando o tamanho do sistema colidente diminui (colisões $p p$ e $p(d) A)$ a descrição hidrodinâmica deveria, a priori, deixar de ser válida. Essa é uma questão em aberto e vem sendo bastante investigada nos últimos anos, principalmente porque modelos apoiados na abordagem no CGC também fornecem uma descrição qualitativamente satisfatória do mesmo conjunto de dados experimentais descritos pela abordagem hidrodinâmica [140,160,162,163, 167-177].

Assim como o efeito ridge, o entendimento quanto as chamadas "assimetrias azimutais" que têm sido observadas em colisões $p P b$ nas energias do LHC pelas colaborações ALICE [179], ATLAS [181-183] e CMS [153,184], também se encontra sujeito a diferentes explicações teóricas [82,141-148, 165,380-383]. Este ponto será desenvolvido em maiores detalhes mais adiante, no próximo capítulo desta tese.

Em [375], os autores dessa referência estudaram a dependência do momento transversal médio de parículas carregadas com a rapidez utilizando a fatorização $k_{T}$ e argumentos bastante gerais que levaram a expressões analíticas igualmente simples. O modelo de dipolo GBW [113] foi utilizado para descrever a distribuição não integrada de gluons, mas, no entanto, a conversão dos pártons interagentes nos hádrons observados no estado final da colisão, realizada por funções de fragmentação, não foi implementada.

Considerando colisões $p P b$ a $5.02 \mathrm{TeV}$, esses autores mostraram que o momento transversal médio de partículas carregadas, $\left\langle p_{T}(\sqrt{s}, y)\right\rangle$, cresce com a rapidez quando comparado ao seu valor em $y=0$ na abordagem do CGC. Esse resultado está de acordo com o esperado a nível qualitativo, uma vez que na teoria efetiva do CGC o momento transversal médio é dado pela escala de saturação do núcleo $\left(Q_{s, A}^{2} \sim Q_{0}^{2} N_{\text {part }} e^{\lambda y}\right.$, onde $Q_{0}^{2}$ e $\lambda$ assumem valores constantes e $N_{\text {part }}$ representa o número de partículas participantes em uma colisão $p A$ [35-43]), que, por sua vez, cresce a medida que $y$ aumenta. 
Como pode ser visto na Figura 4.17, este comportamento é totalmente diferente do esperado (nas mesmas condições) considerando-se a abordagem hidrodinâmica, onde $\left\langle p_{T}(\sqrt{s}, y)\right\rangle$ decresce devido à diminuição da multiplicidade de partículas produzidas.

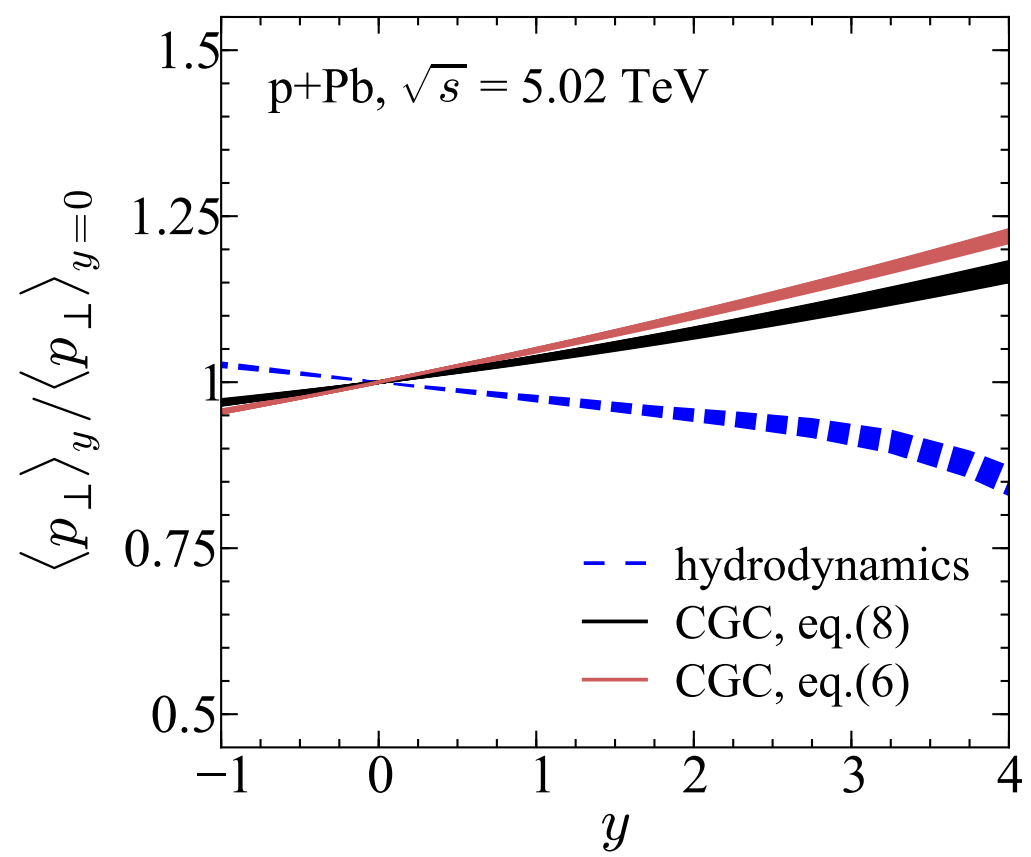

Figura 4.17: Momento transversal médio de partículas produzidas em colisões $p P b$ em função da rapidez, dividido pelo valor do momento transversal médio em $y=0$. Os resultados utilizando-se duas aproximações diferentes no formalismo do CGC, equações (6) e (8) de [375], diferem qualitativamente dos resultados obtidos considerando-se a abordagem hidrodinâmica. Figura extraída de [375].

A predição destes dois comportamentos distintos foi uma grande motivação para uma análise detalhada da dependência do momento transversal médio com a energia e a rapidez de partículas carregadas nessas colisões. Procurando verificar o quão realista é a conclusão apresentada nessa referência, um estudo mais completo deste observável utilizando a teoria efetiva do CGC é apresentado a seguir. Os resultados deste encontram-se publicados na referência [80].

No formalismo do CGC os hádrons são descritos pelas distribuições não integradas de gluons que, por sua vez, são obtidas através dos modelos de dipolo, $\mathcal{N}(x, r)$. A exemplo dos resultados apresentados para a produção líquida de bárions em colisões $A A[78]$ (vide Figura 4.3), o modelo GBW não é capaz de descrever o espectro de 
momento transversal de partículas carregadas medido em colisões $p p$ e $p A$, já que esse observável também não apresenta uma dependência exponencial com o aumento de $p_{T}[384-390]$ tal como a produzida por este modelo. Por este motivo, dois outros modelos fenomenológicos para a amplitude de espalhamento dipolo-alvo foram considerados neste estudo.

Analisando-se os resultados de [375], compilados na Figura 4.17, vê-se que a diferença entre os resultados obtidos com CGC e hidrodinâmica vai ficando mais acentuada conforme a rapidez aumenta em direção à região de fragmentação do próton $(y>0)$. Conforme mencionado no capítulo 2 e na seção 4.1, a produção de hádrons na região frontal de rapidez $(y \gtrsim 2)$ em colisões hadrônicas é um típico exemplo de processo do tipo "diluído-denso", processo no qual a função de onda do projétil é provada na região de altos valores de $x_{1}$. Nessa situação o regime do sistema partônico formado é diluído e pode ser descrito pelas equações de evolução usuais da QCD, enquanto que a função de onda do alvo é provada na região de pequenos valores de $x_{2}$, onde espera-se a formação do CGC devido à existência de um meio partônico altamente denso. Nessas circunstancias, a presença de efeitos não lineares da QCD alteram as equações de evolução, tornando-as igualmente não lineares.

Assim como nas seções 4.2 e 4.3, é natural considerar o formalismo híbrido para o estudo da dependência do momento transversal médio de partículas produzidas em colisões hadrônicas com a rapidez de posse que a seção de choque invariante para a produção inclusiva de hádrons em processos hadrônicos pode ser descrita no formalismo do CGC pela equação (4.4).

As quantidades $\mathcal{N}_{\mathcal{A}, \mathcal{F}}(x, r)$ na equação acima mencionada carregam toda informação sobre o espalhamento hadrônico e, consequentemente, sobre os efeitos quânticos e não lineares na função de onda hadrônica, e podem ser obtidas resolvendo-se a equação de evolução BK [44-47], que é a equação de evolução mais simples para a amplitude de espalhamento dipolo-hádron no regime de saturação (uma aproximação de campo médio para primeira equação da hierarquia de equações JIMWLK [35-40,44-47]). Diversos estudos sobre a solução da equação BK com a inclusão de correções de mais 
altas ordens em $\alpha_{s}$ foram realizados nos últimos anos [200,391,392] e, posteriormente, estas soluções foram utilizadas como ingrediente nas análises da produção de hádrons em colisões $p p$ e $p A$ onde obteve-se uma descrição muito boa dos dados experimentais $[166,300,393,394]$.

No que se segue, ao invés de nos utilizarmos da solução da equação BK, consideramos dois modelos de dipolo fenomenológicos (apresentados na seção 2.3.4), a saber, os modelos BUW e DHJ, cujas construções são baseadas em soluções analíticas dessa equação, que nos permite investigar a possibilidade de se obter uma primeira indicação de se os dados do LHC para o espectro de momento transversal de partículas carregadas são ou não sensíveis à violação do scaling geométrico na região de altos valores de $p_{T}$. Além disso, é importante salientar que os modelos de dipolo escolhidos diferem do modelo GBW, apresentando uma a dependência da dimensão anômala com o momento transversal e, por conta disso, abrem ambiente para o esclarecimento da origem das diferenças entre as diversas previsões encontradas na literatura. Tal análise se torna uma difícil tarefa quando a solução numérica da equação BK é utilizada como ingrediente nos os cálculos aqui apresentados.

É importante mencionar que a abordagem fenomenológica utilizada neste estudo possui limitações, sendo válida apenas em uma região restrita de momento transversal $\left(p_{T} \lesssim 20 \mathrm{GeV}\right)$ e não é competitiva com relação a parametrizações mais recentes que foram utilizadas para descrever o fator de modificação nuclear, $R_{p A}[166,299$, 300,393-395]. Como mostrado nessas referências, um tratamento mais preciso da geometria nuclear e/ou das condições iniciais é necessário para que esse observável seja descrito satisfatoriamente, porém tal tarefa encontra-se além dos objetivos de nossa investigação e tais pontos não serão tratados no que se segue. Por outro lado, o comportamento de $\left\langle p_{T}\right\rangle$ parece ser determinado pela região de pequenos valores de momento transversal e o uso de modelos fenomenológicos que descrevem os dados experimentais nessa região cinemática são capazes de fornecer predições realísticas para esta quantidade.

O momento transversal médio, $\left\langle p_{T}\right\rangle$, das partículas produzidas em colisões hadrônicas 
é definido como

$$
\left\langle p_{T}\right\rangle=\frac{\int d^{2} p_{T} p_{T} \frac{d N_{h}}{d y d^{2} p_{T}}}{\int d^{2} p_{T} \frac{d N_{h}}{d y d^{2} p_{T}}}
$$

que obviamente depende da rapidez dessas partículas, isto é, $\left\langle p_{T}\right\rangle \equiv\left\langle p_{T}(y, \sqrt{s})\right\rangle$ e também do limite inferior de integração no momento transversal, $p_{T, \min }$.

Visando fornecer predições realísticas para este observável nas energias do LHC é fundamental utilizar um modelo que descreva os dados experimentais para o espectro de momento transversal das partículas produzidas. A primeira etapa, portanto, consiste em comparar as predições para esse espectro utilizando-se os modelos BUW e DHJ com os recentes dados do LHC.

Além dos modelos de dipolo mencionados, nessa comparação utilizamos a distribuição de pártons CTEQ5L [91] e a função de fragmentação KKP na equação (4.4). A massa do hádron produzido foi escolhida como sendo o valor médio da soma das massas do píon, do kaon e do próton. Nossos cálculos referem-se a valores centrais de $\eta$ na região de pseudorapidez considerada pelos experimentos e colisões do tipo minimum bias, onde tem-se que $A \equiv A_{\text {min. bias }}=20$ (18.5) para colisões $p P b(d A u)$ [205].

A Figura 4.18 apresenta a comparação dos resultados obtidos com o uso dos modelos de dipolo BUW e DHJ, considerando-se os parâmetros originais destes modelos, rotulados por "BUW - old" e "DHJ - old", com os dados do LHC para o espectro em $p_{T}$ de partículas carregadas produzidas em colisões $p P b$ em $\sqrt{s}=5.02 \mathrm{TeV}$ e diferentes valores de rapidez [297]. Como pode ser observado, estes modelos não são capazes de descrever os dados da colaboração ALICE [297] na região de grandes momentos transversais com seus parâmetros originais. Por essa razão, como um procedimento natural averiguamos se um novo ajuste dos parâmetros livres desses modelos poderia melhorar a descrição destes dados experimentais.

Como ponto de partida os parâmetros livres das amplitudes de espalhamento dos modelos BUW e DHJ foram fixados de modo a descreverem a distribuição de momento transversal de partículas produzidas em colisões $p P b$ a $\sqrt{s}=5.02 \mathrm{TeV}$. Na 


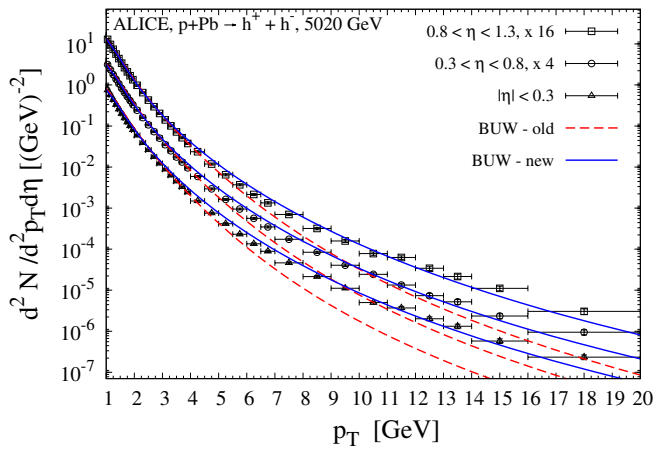

(a)

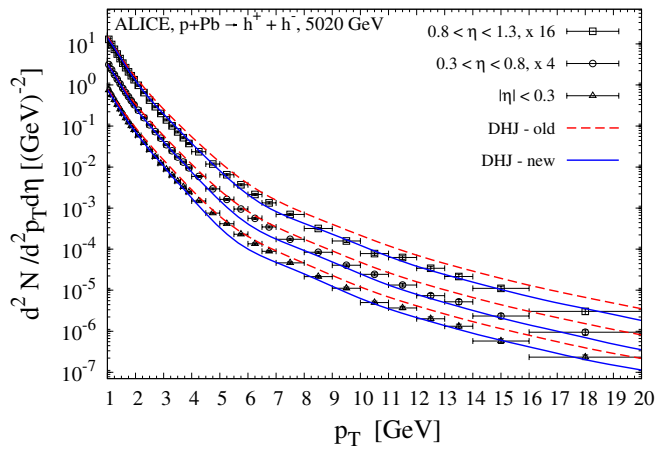

(b)

Figura 4.18: Comparação entre as predições dos modelos (a) BUW e (b) DHJ para o espectro de momento transversal de partículas carregadas produzidas em colisões $p P b$ e os dados da colaboração ALICE [297]. Para a nova versão do modelo $B U W$ assumiu-se $K=3.7$ para todas as janelas de pseudorapidez enquanto que para a nova versão do modelo DHJ $K=3.0,3.0$ e 3.7 para $\langle\eta\rangle=0$, 0.55 e 1.05 , respectivamente.

sequência, utilizando-nos dos mesmos modelos e parâmetros assim fixados, comparamos nossos resultados aos dados experimentais medidos em colisões $p p$ em outras energias e intervalos de rapidez, porém assumindo que a quantidade $\gamma_{s}$ que figura na dimensão anômala (o parâmetro $\gamma\left(x, r^{2}\right)$ que figura nas equações $(2.29)$ e (2.30)) fosse um parâmetro livre a ser ajustado para descrever satisfatoriamente tais dados ${ }^{11}$.

Os resultados obtidos para colisões $p p$ denotados por "BUW - new"e "DHJ - new", são apresentados nas Figuras 4.18 (a) e 4.18 (b), respectivamente, com a fixação dos seguintes parâmetros: $a=2,0, b=125$ e $\gamma_{s}=0,74$ para o modelo BUW; $d=1,0$ e $\gamma_{s}=0,7$ para o modelo DHJ. Como pode ser observado, os dados são melhor descritos assumindo-se valores maiores de $\gamma_{s}(\geq 0,7)$, o que é consistente com os resultados obtidos através da equação BFKL em ordem seguinte à dominante com acoplamento fixo [396, 397].

Com a adoção desses parâmetros os modelos BUW e DHJ se ajustam qualitativamente bem aos dados experimentais. Na região de $4<p_{T}<7 \mathrm{GeV}$ o modelo DHJ apresenta uma espécie de "arco", cujo comportamento é uma reminescência da

\footnotetext{
${ }^{11}$ Segundo as referências [204,205], os parâmetros originais para os modelos de dipolo utilizados aqui num primeiro momento fixam essa quantidade em $\gamma_{s} \sim 0,63$, valor obtido a partir da equação BFKL em ordem dominante.
} 
transformada de Fourier que é calculada numericamente ${ }^{12}$. A despeito dessas flutuações numéricas é importante ressaltar que o momento transversal médio é afetado apenas marginalmente por essas pequenas oscilações e os ajustes qualitativos apresentados aqui para os modelos de dipolo considerados é suficiente para fornecer predições realísticas para este observável, já que as contribuições mais relevantes vêm da região de pequenos momentos transversais.

Uma vez fixados novos parâmetros para os modelos BUW e DHJ através dos dados experimentais para o espectro de momento transversal de hádrons produzidos em colisões $p P b$, pode-se agora comparar as predições desses modelos com os recentes dados experimentais do LHC para o espectro de momento transversal de partículas carregadas e píons neutros produzidos em colisões $p p$ em diferentes valores de energia e intervalos de rapidez. Neste caso, o único parâmetro livre de nossas predições é o fator $K$, que pode ser dependente da energia e da rapidez ${ }^{13}$. Esse parâmetro será fixado de modo a tornar possível a descrição dos dados experimentais na região de pequenos valores de momento transversal. Os resultados obtidos encontram-se apresentados nas Figuras 4.19 e 4.20, onde é possível observar que ambos os modelos descrevem os dados experimentais razoavelmente bem na região de pequenos valores de $p_{T}$. Além disso, tem-se que as predições com o modelo BUW se tornam piores com o aumento do valor de $p_{T}$ conforme a energia do centro de massa aumenta. Em contraste, o modelo DHJ também é capaz de descrever esta mesma região razoavelmente bem, fato que pode ser associado à contribuição de violações do scaling geométrico considerados neste modelo.

Na Figura 4.19 (a) os fatores $K=2.47(K=2.3), 2.07$ (1.85) e 1.77 (1.6) em $\sqrt{s}=0.9,2.76$ e $7 \mathrm{TeV}$, respectivamente, foram utilizados para o modelo BUW (DHJ). Já na Figura 4.19 (b) assumiu-se $K=3.3,2.5$ e 2.3 para ambos os modelos. Na Figura 4.20 (a), os fatores $K=3.0,2.5$ e 2.3 para $\sqrt{s}=0.9,2.36$ e $7 \mathrm{TeV}$, respectivamente,

\footnotetext{
${ }^{12}$ A existência desse comportamento já era conhecida e foi discutida em [204].

${ }^{13}$ Como será visto adiante, nossos resultados para o comportamento de $\left\langle p_{T}\right\rangle$ com a rapidez serão independentes deste parâmetro já que trabalharemos com razões como as apresentadas na Figura 4.17 .
} 


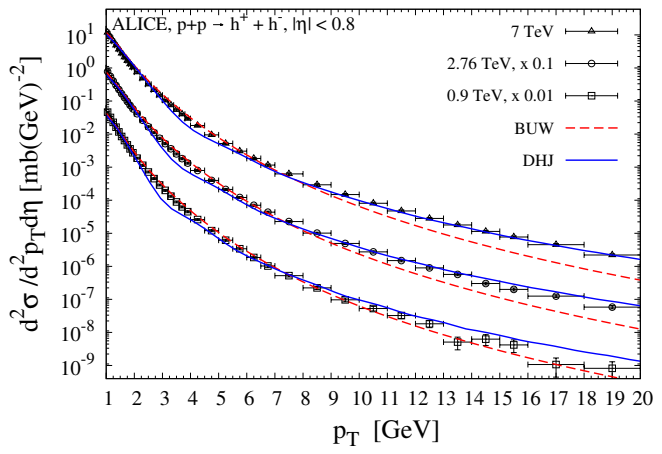

(a)

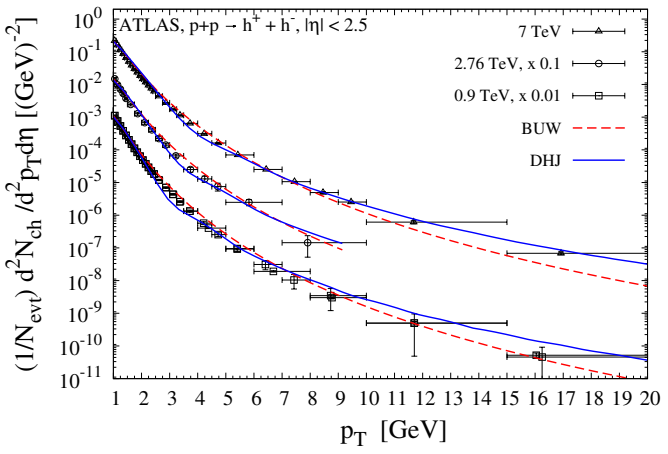

(b)

Figura 4.19: Resultados dos modelos BUW e DHJ para o espectro de momento transversal de partículas carregadas em colisões pp. (a) Comparação com os dados da colaboração ALICE [385]. (b) Comparação com os dados da colaboração ATLAS [386, 387].

foram utilizados para ambos os modelos. Por fim, na Figura 4.20 (b) foram utilizados os fatores $K=1,2$ e 2, 0 para as energias $\sqrt{s}=0.9$ e $7 \mathrm{TeV}$, respectivamente.

Finalmente, enquanto uma última verificação, nas Figuras 4.21 (a) e (b) são apresentadas as comparações dos modelos BUW e DHJ (levando em consideração a fixação dos parâmetros acima mencionada) com os dados para o espectro de momento transversal de hádrons carregados e neutros nas regiões central e frontal em colisões $p p$ e $d A u$ nas energias do RHIC. É possível observar que, como antes, ambos os modelos descrevem bem os dados experimentais na região frontal. Na região central de rapidez, por outro lado, o modelo BUW descreve bem os dados experimentais de colisões $p p$ para $p_{T} \leq 10 \mathrm{GeV}$, mas falha para $p_{T} \geq 3 \mathrm{GeV}$ no caso de colisões $d A u$. Em contraste, os resultados com o modelo DHJ não são mostrados nessa região de rapidez pois são altamente afetados por oscilações para $p_{T} \gtrsim 5 \mathrm{GeV}$. A falha na descrição dos dados experimentais na região central de rapidez nas energias do RHIC não é uma surpresa já que a energia da colisão não é muito grande e o formalismo aqui empregado é adequado para o estudo de colisões assimétricas, tais as que ocorrem na região frontal de rapidez, onde a função de onda do alvo é provada na região de pequenos valores de $x$.

Finalmente, na Figura 4.22 é demonstrado que a nova versão do modelo BUW também satisfaz a propriedade de scaling geométrico e é capaz de descrever os dados 


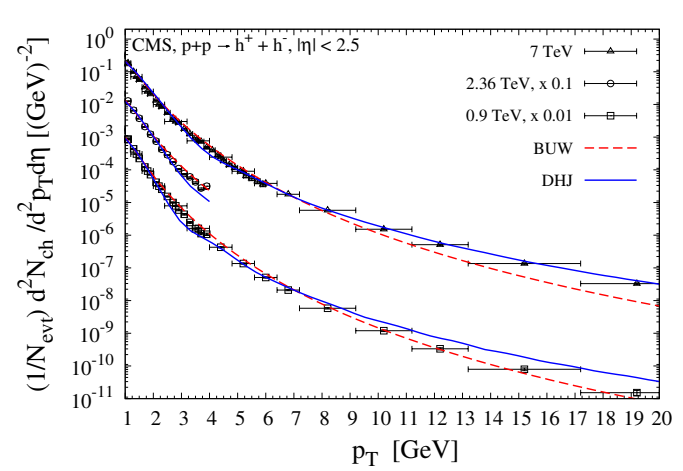

(a)

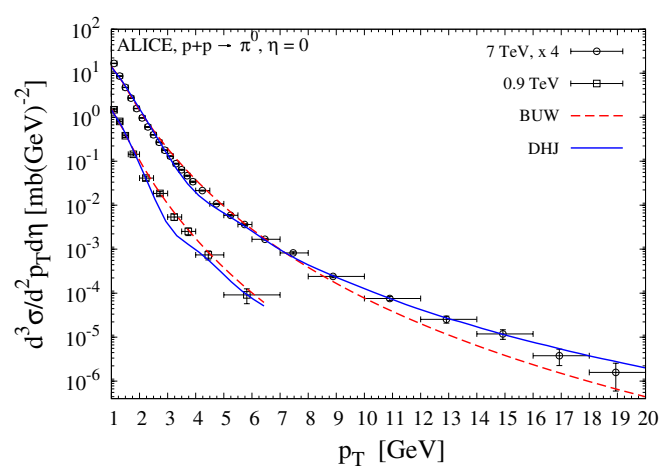

(b)

Figura 4.20: Resultados dos modelos BUW e DHJ para (a) o espectro de momento transversal de partículas carregadas em colisões pp comparados com os dados da colaboração CMS [388-390] e (b) para o espectro de momento transversal de píons neutros produzidos em colisões $p p$ comparados com os dados da colaboração ALICE [398].

da seção de choque total $\gamma^{*} p$ medidos em colisões ep no HERA para uma extensa região de virtualidades do fóton.

Os resultados apresentados nas Figuras 4.18 - 4.21 permitiu-nos obter resultados realísticos para o momento transversal médio em colisões $p p$ e $p P b$ e nos encorajou traçar sua dependência com a energia e a rapidez através da razão

$$
R=\frac{\left\langle p_{T}(y, \sqrt{s})\right\rangle}{\left\langle p_{T}(0, \sqrt{s})\right\rangle}
$$

onde o denominador representa o momento transversal médio das partículas produzidas para rapidez nula.

A motivação para o estudo dessa razão é que ela reduz as incertezas nos cálculos efetuados, tais como as relacionadas às funções de fragmentação e à escolha do momento transversal mínimo presente no cálculo de $\left\langle p_{T}\right\rangle$. No que se segue analisamos a dependência das nossas predições com relação aos modelos utilizados, assim como o impacto da inclusão das funções de fragmentação.

Na Figura 4.23 comparamos as predições dessa razão obtidas com os modelos BUW e DHJ com aquelas obtidas com o modelo GBW onde assumimos, em todos os casos, o valor $p_{T, \text { min }}=1 \mathrm{GeV}$ para o momento transversal mínimo que figura na equação 


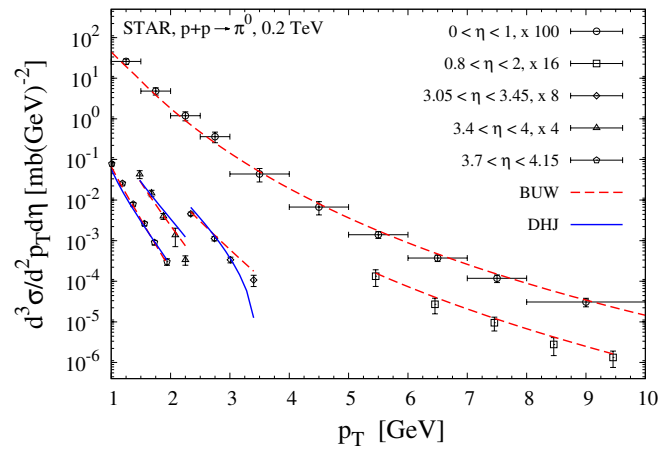

(a)

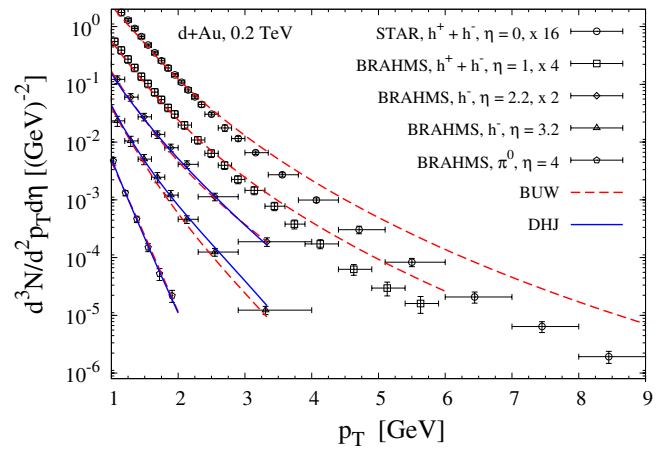

(b)

Figura 4.21: (a) Resultados dos modelos BUW e DHJ para o espectro de momento transversal de píons neutros produzidos em colisões $\mathrm{pp}$. Os fatores $K=1.5$ para $\langle\eta\rangle=0.5$ e $K=1.2$ para $\langle\eta\rangle=1.4,3.25,3.7,3.925$ foram utilizados para os resultados com o modelo BUW. Para o modelo DHJ utilizou-se $K=1.2$ para $\langle\eta\rangle=3.25,3.7,3.925$. Os dados experimentais são da referência [399]; (b) Resultados dos modelos BUW e DHJ para o espectro de momento transversal de hádrons produzidos em colisões $d A u$. Os fatores $K=2,9,2,5,2,0,1,0$ e 1, 0 for $\eta=0,1,2,2,3,2$ e 4, respectivamente, foram utilizados nos resultados com o modelo BUW. Para o modelo DHJ tem-se que $K=2,5,2,4,1,5$ para $\eta=2,2,3,2$ e 4, respectivamente. Os dados experimentais são da referência [400-402].

(4.17). É importante mencionar, uma vez mais, que o modelo GBW não é capaz de descrever os dados experimentais para o espectro de momento transversal de hádrons produzidos em colisões hadrônicas, já que nesse modelo a amplitude de espalhamento de dipolo decresce exponencialmente para grandes valores do momento transversal. Todavia, como este modelo é usualmente considerado para a obtenção de resultados analíticos para diversos observáveis, é relevante verificar se suas predições para $\left\langle p_{T}\right\rangle$ são realísticas.

As Figuras 4.23 (a) e (c) apresentam as predições para a razão apresentada na equação (4.18) desconsiderando-se as funções de fragmentação ${ }^{14}$ enquanto que nos painéis (b) e (d) a fragmentação dos pártons nos hádrons observados experimentalmente é levada em consideração. É oportuno mencionar que os resultados com o modelo GBW sem a inclusão da fragmentação, obtidos aqui com o uso do formalismo

\footnotetext{
${ }^{14}$ Neste caso, tem-se que $D_{h / f}(z, \mu)=\delta(1-z)$, com $f=q, \bar{q}, g$, na equação (4.4) e, portanto, o momento transversal médio hadrônico coincide com o momento transversal médio partônico, ou seja, $\left\langle p_{T}\right\rangle=\left\langle q_{T}\right\rangle$.
} 


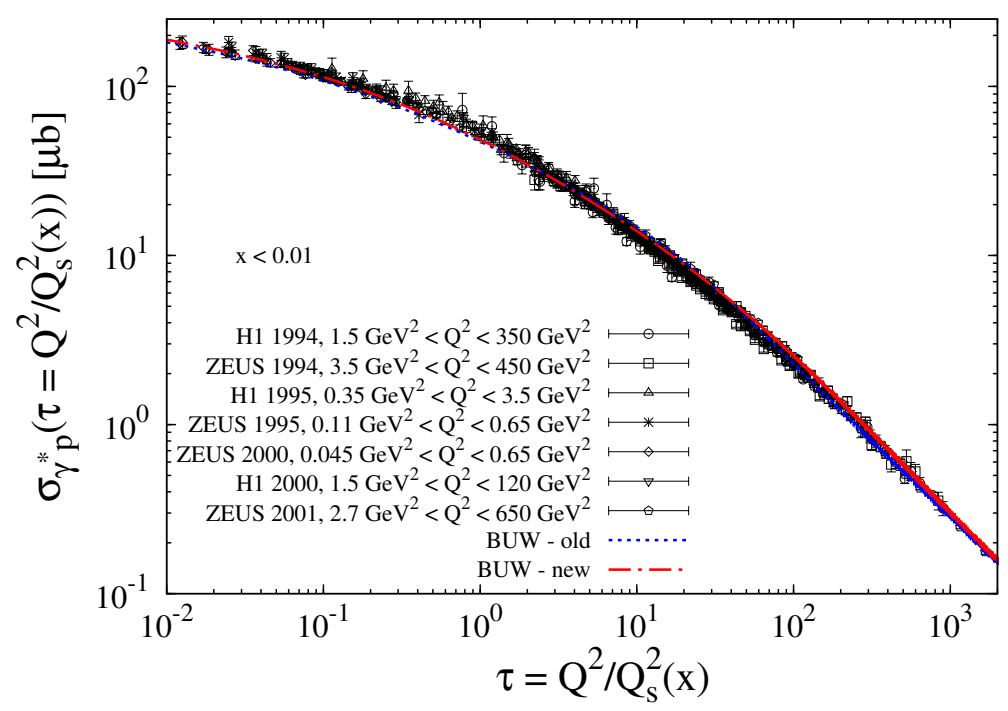

Figura 4.22: Comparação entre o resultado do modelo BUW (com os parâmetros originais da referência [205] e os parâmetros novos) e os dados experimentais para a seção de choque total $\gamma^{*} p[23,24,27,30,32,403,404]$ medidos a partir de colisões ep no HERA.

híbrido, são similares àqueles obtidos na referência [375] com a fatorização $k_{T}$.

Nessas figuras é possível observar que o uso dos modelos BUW e DHJ fornecem um comportamento semelhante para essa razão (quando comparados entre si) porém diferem significativamente do comportamento quando o modelo GBW é utilizado: enquanto o modelo GBW prediz um crescimento da razão estudada para $y \leq 6$, os modelos BUW e DHJ predizem que esta mesma razão é quase constante ou decresce com a rapidez. Por outro lado, a inclusão da fragmentação dos pártons em hádrons modifica a dependência com a rapidez dessa razão, implicando em um menor crescimento para a predição com o modelo GBW. No caso das predições com os modelos BUW e DHJ, a inclusão da fragmentação implica que a queda da razão considerada começa em valores menores de rapidez.

Essa simples análise mostra que a inclusão da fragmentação dos pártons em hádrons possui um importante impacto no comportamento de $\left\langle p_{T}\right\rangle$. Todavia, a principal diferença entre nossas predições e aquelas apresentadas na referência [375] vem do modelo utilizado para descrever a dinâmica da QCD a altas energias, isto é, dos modelos de 


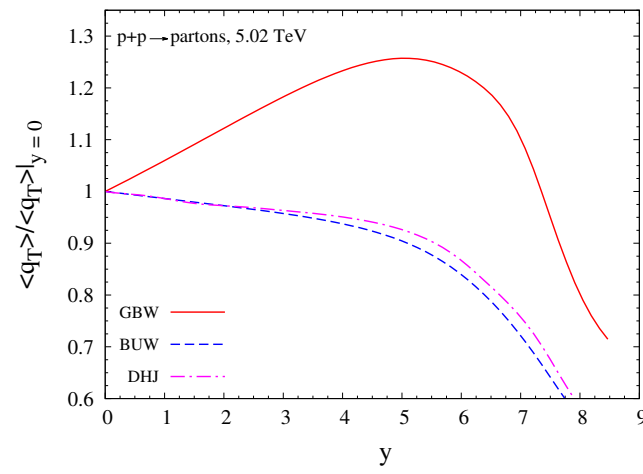

(a)

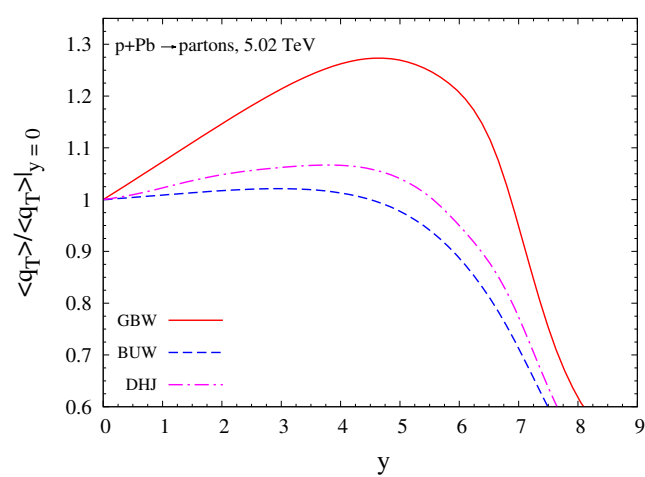

(c)

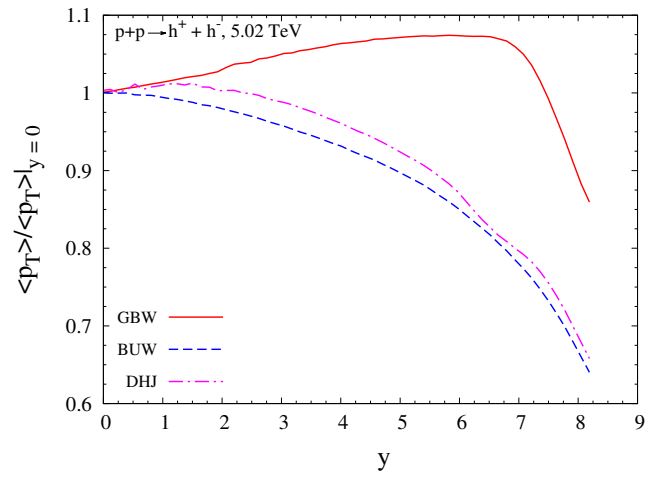

(b)

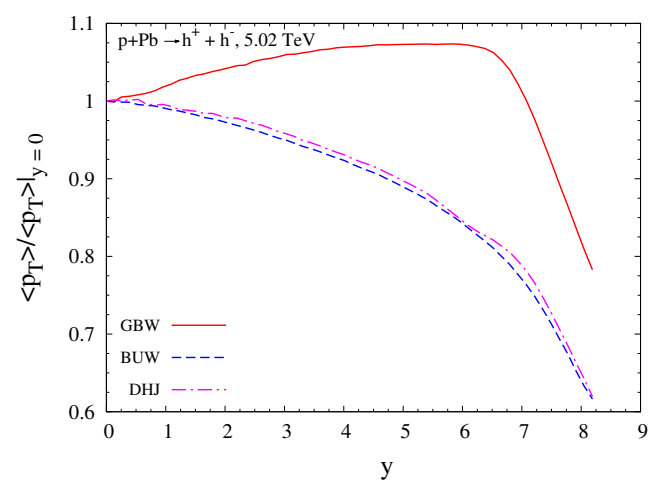

(d)

Figura 4.23: Dependência da razão $\left\langle p_{T}(y, \sqrt{s})\right\rangle /\left\langle p_{T}(0, \sqrt{s})\right\rangle$ com relação à amplitude de espalhamento frontal dipolo-hádron em colisões $p p$ e $p P b$. Os resultados dos painéis (a) e (c) foram obtidos desconsiderando-se o processo de fragmentação enquanto os resultados apresentados nos painéis (b) e (d) incluem este processo através da distribuição KKP.

dipolo considerados. Além disso, o comportamento dessa razão a altíssimos valores de rapidez, é determinado por restrições cinemáticas associadas à limitação do espaço de fase uma importante característica não presente nos resultados da referência [375] (os resultados apresentados nessa referência, e rotulados como "CGC" (vide Figura 4.17), crescem indefinidamente com a rapidez).

Todos os resultados para a razão $R$, equação (4.18), apresentados até aqui foram obtidos com $p_{T, \text { min }}=1 \mathrm{GeV}$. Na Figura 4.24 a dependência dos nossos resultados com este corte arbitrário no momento transversal é analisada. Para estes cálculos comparou-se o valor da escala de saturação para um dado valor de momento transversal e rapidez com o valor correspondente de $p_{T}$ e assumiu-se que a escala de fatorização, 


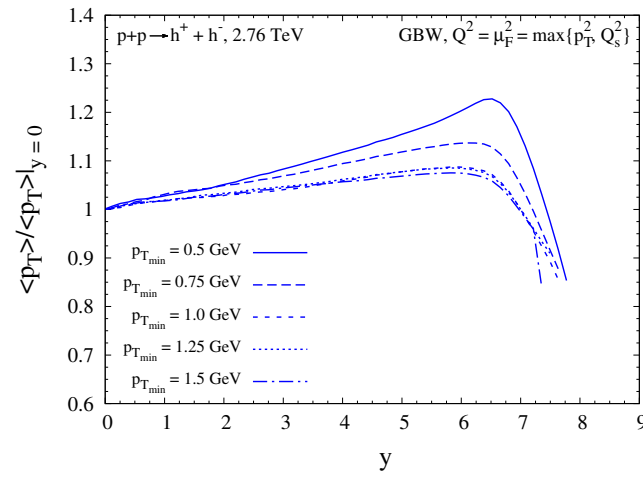

(a)

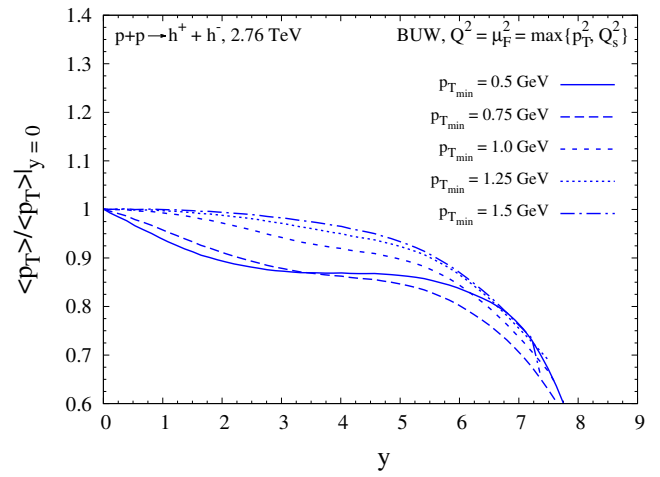

(b)

Figura 4.24: Dependência da razão $\left\langle p_{T}(y, \sqrt{s})\right\rangle /\left\langle p_{T}(0, \sqrt{s})\right\rangle$ com o valor do momento transversal mínimo, $p_{T, \text { min }}$, considerando (a) o modelo $G B W$ e (b) o modelo BUW para a amplitude de espalhamento frontal.

$Q^{2}$, que figura nas distribuições de pártons, $x f\left(x, Q^{2}\right)$, é dada pela maior escala ${ }^{15}$. No entanto, resultados semelhantes podem ser obtidos "congelando-se" a escala de fatorização no valor mínimo de $Q^{2}$ permitido pelas distribuições de pártons e funções de fragmentação utilizadas quando menores valores de $p_{T}$ são provados nos cálculos. Os resultados apresentados na Figura 4.24 indicam que o comportamento da razão $R$ com a rapidez não é fortemente modificado pela escolha de $p_{T, \min }$ e, por esse motivo, mantivemos o valor adotado nos resultados a seguir.

A Figura 4.25 apresenta o comportamento da razão $\left\langle p_{T}(y, \sqrt{s})\right\rangle /\left\langle p_{T}(0, \sqrt{s})\right\rangle$ em colisões $p p$ e $p P b$ para diferentes energias do centro de massa da colisão. Como resultado, vê-se que as predições com os modelos BUW (linhas azuis) e DHJ (linhas vermelhas) são semelhantes, porém com aqueles obtidos com o modelo DHJ sendo ligeiramente maiores que aqueles obtidos com o modelo BUW. Para além do fato dessa razão crescer com a energia, para um valor de rapidez fixo, pode-se observar que esta razão é maior para colisões $p p$ em comparação com colisões $p P b$ para um dado valor de energia, como já parece sugerir a Figura 4.26. Esses resultados indicam que a altíssimas energias as predições para a razão $R$ em colisões $p p$ e $p P b$ se tornam iguais demarcando

\footnotetext{
${ }^{15}$ Esta suposição foi utilizada na referência [405] a fim de se estender o formalismo híbrido para a produção de hádrons na região de $p_{T}$ muito pequeno, obtendo-se uma descrição muito boa dos dados da colaboração LHCf.
} 


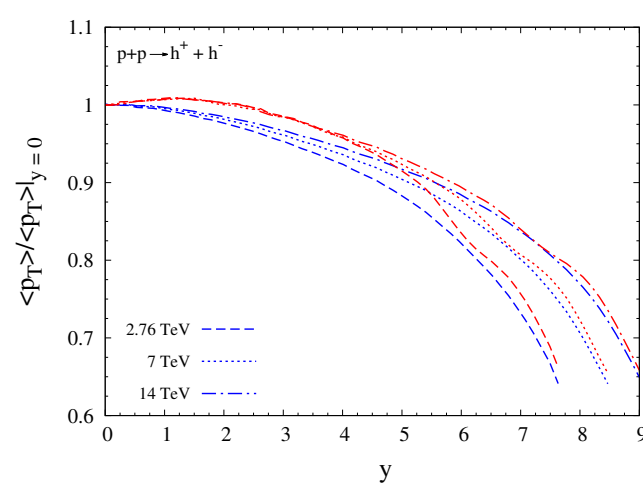

(a)

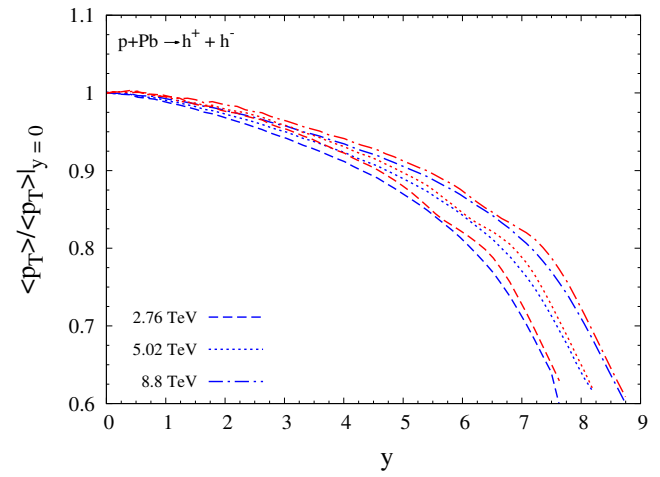

(b)

Figura 4.25: Dependência com a rapidez da razão $R=\left\langle p_{T}(y, \sqrt{s})\right\rangle /\left\langle p_{T}(0, \sqrt{s})\right\rangle$ em (a) colisões $p p$ e (b) colisões $p P b$ para diferentes energias. As predições com o modelo BUW (DHJ) são representadas pelas curvas azuis (vermelhas).

um importante teste para o formalismo híbrido e, portanto, para o cenário do CGC.

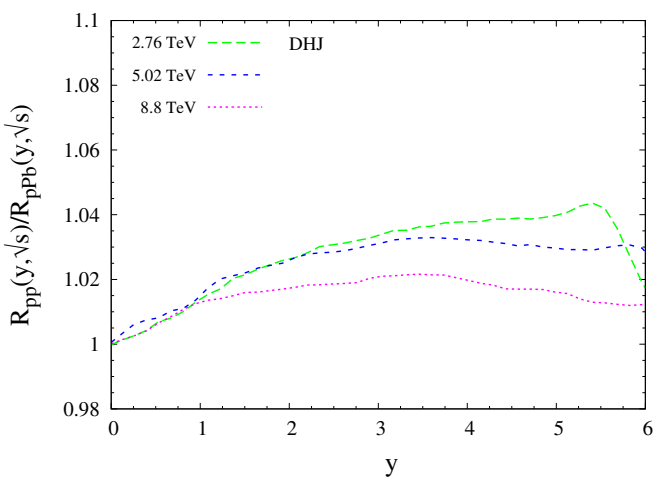

(a)

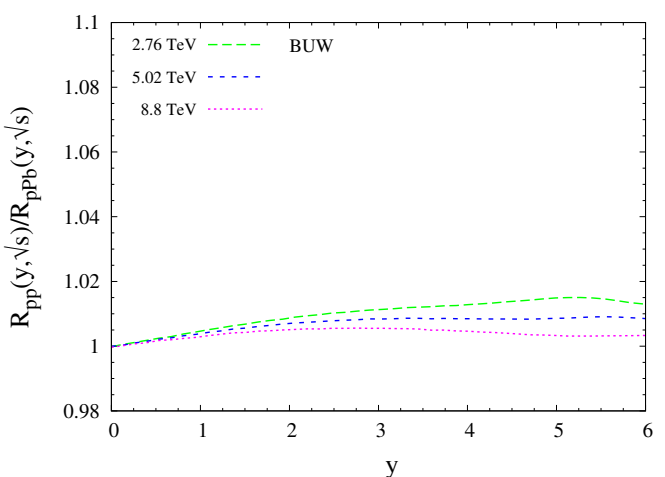

(b)

Figura 4.26: Dependência com a rapidez da razão entre as predições para $R=$ $\left\langle p_{T}(y, \sqrt{s})\right\rangle /\left\langle p_{T}(0, \sqrt{s})\right\rangle$ em colisões $p p$ e colisões $p P b$ considerando os modelos de dipolo (a) DHJ e (b) BUW.

À luz dos resultados apresentados, acreditamos que a análise da razão $R$ em colisões $p p$ e $p P b$ pode ser útil para provar a dinâmica da QCD na região frontal de rapidez. Os resultados apresentados na Figura 4.25 indicam que esta razão decresce com a rapidez para colisões $p P b$ para energias do LHC, apresentando um comportamento semelhante ao obtido através da abordagem hidrodinâmica (vide curva azul na Figura 4.17), o que implica que este observável, em princípio, quando analisado isoladamente, não pode ser utilizado para discriminar as abordagens do CGC e da hidrodinâmica na descrição 
de eventos de altas multiplicidades.

Esta conclusão é oposta àquela obtida na referência [375], cujas diferenças residem aos seguintes fatores: em primeiro lugar, os resultados rotulados como "CGC" na referência [375] foram obtidos utilizando-se aproximações analíticas para uma distribuição não integrada de gluons específica que não é capaz de descrever (nem a nível qualitativo) o espectro de momento transversal medido experimentalmente; em segundo lugar, os resultados obtidos via física do CGC e apresentados em [375] não incluem a importante contribuição do processo de fragmentação no cálculo do momento transversal médio. Em terceiro e último lugar, as restrições cinemáticas associadas às limitações no espaço de fase para altos valores de rapidez não foram incluídas em [375] e, como visto na Figura 4.23, elas desempenham um papel importante mesmo quando o momento transversal médio é calculado a nível partônico.

Em contraste, na análise aqui apresentada, a razão $R$ foi calculada utilizando-se dois diferentes modelos para a amplitude de espalhamento frontal que são capazes de descrever os dados experimentais atuais para o espectro de momento transversal de partículas carregadas e píons neutros produzidos em colisões $p p$ e $p P b$ no LHC. Além disso, os efeitos da fragmentação dos pártons em hádrons e as restrições no espaço de fase também foram incluídos. Embora tenhamos utilizado o formalismo híbrido ao invés da fatorização $k_{T}$, verificamos que ambas as abordagens fornecem um comportamento semelhante quando o modelo GBW é considerado e os efeitos da fragmentação dos pártons não são levados em consideração. Sendo assim, nossos resultados para a razão $R$ demonstram que a principal diferença entre os resultados aqui apresentados vem do tratamento da dinâmica da QCD a altas energias.

Com o uso do formalismo da fatorização $k_{T}$, na próxima seção estudamos a produção de partículas em colisões $p p, p A$ e $A A$ na região central de rapidez e estimamos, pela primeira vez, a influência das correções em $\alpha_{s}$ incluídas na seção de choque invariante para a produção de gluons nesse formalismo nas distribuições de rapidez e de multiplicidade de hádrons carregados medidas nessas colisões. 


\subsection{Produção de partículas com a fatorização $k_{T}$}

Nas seções anteriores estudou-se a produção de partículas e a dinâmica da QCD na região frontal de rapidez aplicando-se o formalismo híbrido à produção líquida de bárions, à produção de mésons pesados e também à produção de partículas carregadas e píons neutros nas energias do RHIC e do LHC. Esta seção, no entanto, encontra-se voltada à dinâmica da QCD na região central de rapidez, $|y| \lesssim 2$, até então marginalmente explorada nesta tese. Os resultados aqui apresentados deram origem à publicação [81].

Diferente das situações estudadas anteriormente, em colisões que ocorrem na região central de rapidez, a função de onda do projétil e do alvo são provadas na região de pequenos valores de $x_{1,2}$, onde a fatorização híbrida, apresentada na seção 4.1, aplicada nos estudos mencionados acima deixa de ser válida, pois os efeitos não lineares e múltiplos espalhamentos precisam ser ressomados em ambos hádrons colidentes. Desta forma, conforme discutido em mais detalhes também na seção 4.1, faz-se necessário considerar a fatorização $k_{T}$ para a produção de partículas quando da utilização da teoria efetiva do CGC.

O formalismo da fatorização $k_{T}$ para a produção de gluons na região central de rapidez foi proposto na referência [33] e posteriormente derivado nas aproximações de $\log (1 / x)$ dominante e acoplamento fixo $\left(\alpha_{s}=\right.$ constante $)$ na referência [74], considerandose o espalhamento de um sistema partônico diluído por outro denso.

Em uma série de trabalhos [38-40], Kharzeev, Levin e Nardi (KLN) estudaram a produção de partículas na região de rapidez central em colisões $p p, p A$ e $A A$ em termos da fatorização $k_{T}$. Em seus estudos, os autores assumiram que as propriedades relacionadas à produção de hádrons, tais como sua distribuição de momento transversal e rapidez, são determinadas nos estágios iniciais da colisão pela interação entre gluons com momento transversal da ordem do valor da escala de saturação, $Q_{s}{ }^{16}$.

\footnotetext{
${ }^{16}$ A presença desta escala regulariza o comportamento da distribuição de momento transversal dos pártons ao mesmo tempo que justifica a aplicação de uma abordagem perturbativa para o processo
} 
Após as predições obtidas através da abordagem utilizada por KLN serem qualitativamente confirmadas pelos dados experimentais do RHIC e do LHC [38-40,164] diversos estudos foram focados na melhora do formalismo utilizado visando obter uma descrição quantitativa dos mesmos e de outros dados experimentais. Em particular, diferentes distribuições não integradas de gluons e/ou tratamentos para a geometria nuclear foram considerados nas referências $[134,135,164,166,296,395,406,407]$. Todavia, analisando-se com mais detalhes as predições apresentadas nessas referências, observa-se que elas foram obtidas utilizando-se diferentes valores para as escalas de momento que figuram nas constantes de acoplamento presentes neste formalismo. Embora tal incerteza tenha sido esperada e inerente em relação aos cálculos efetuados em ordem dominante em $\alpha_{s}$ (onde as escalas mencionadas não são conhecidas) a inclusão de correções de ordem mais alta na constante de acoplamento forte na seção de choque para produção de gluons resultou ser uma etapa importante na a obtenção de predições quantitativas, com maior precisão.

$\mathrm{Na}$ referência [76], os autores calcularam correções na constante de acoplamento forte para a seção de choque de produção de gluons em mais baixa ordem utilizando o método de fixação de escala proposto por Brodsky, Lepage e Mackenzie (BLM), detalhado na referência [408]. Como resultado, encontrou-se que a seção de choque obtida pode ser expressa em termos de sete fatores de $\alpha_{s}$, ao invés dos três presentes no cálculo da mesma seção de choque para acoplamento fixo (vide equação (4.2)). Em particular, dois desses acoplamentos dependem da escala de momento, $Q$, com componente complexa, sendo uma a conjugada da outra, implicando em uma seção de choque real, como será visto adiante. Outro importante ponto é que este cálculo fixa a escala de todas as constantes de acoplamento que aparecem na seção de choque, diferente do resultado com acoplamento fixo, onde as escalas ficam indeterminadas.

Baseados nesse resultado, que foi formalmente derivado apenas para $y=0$, os autores de [76] propuseram uma conjectura para a forma funcional da seção de choque invariante da fatorização $k_{T}$ com correções em $\alpha_{s}$, onde espera-se que seja válida no de interação. 
mesmo regime de aplicabilidade da fórmula original, derivada em ordem dominante, na qual, no entanto, o acoplamento é fixo. Essa é uma questão em aberto atualmente e embora apenas cálculos numéricos de cunho quantitativo sejam capazes de responder tais questões (sobre a validade e o regime de aplicabilidade da fórmula proposta) definitivamente, os resultados apresentados nas próximas seções, embora possuam cunho qualitativo, podem ajudar a apontar uma direção para etapas futuras.

Embora uma prova formal da conjectura para a seção de choque invariante da produção de gluons corrigida em $\alpha_{s}$ no formalismo da fatorização $k_{T}$, mencionada acima, também seja uma questão em aberto, é esperado que tal conjectura possa ser uma boa aproximação para a resposta exata. Tal expectativa motiva a análise fenomenológica desenvolvida na próxima subseção desta tese.

A seguir comparam-se as predições para a distribuição de rapidez de partículas carregadas produzidas nas energias do LHC e também as predições para o comportamento da multiplicidade de partículas carregadas na região de pseudorapidez nula com a energia obtidas de três maneiras diferentes: a partir da seção de choque invariante da produção de gluons da fatorização $k_{T}$ corrigida em $\alpha_{s}$ (apresentada adiante) e a partir da mesma quantidade, porém sem as correções em $\alpha_{s}$ (equação (4.2)), assumindo-se $i$ ) um acoplamento fixo e $i i$ ) duas diferentes prescrições para as escalas de momento que figuram na constante de acoplamento da mesma maneira em que $[134,135,164,166,296,395,406,407,409]$.

\subsubsection{Produção inclusiva de gluons no formalismo da fato- rização $k_{T}$}

Essa seção inicia-se com uma discussão sobre a produção inclusiva de gluons na região central de rapidez na teoria efetiva do CGC que vai além da apresentada na seção 4.1. Contudo, existe um importante comentário a ser feito antes de apresentarmos as principais expressões a serem utilizadas. Como descrito na seção 4.1, a 
seção de choque para o processo mencionado foi proposta na referência [33] e provado em [74] considerando-se o espalhamento de um sistema "diluído-denso", a constante de acoplamento fixa na aproximação de que apenas as divergências em $\log (1 / x)$ são ressomadas. Consequentemente, sua aplicação é bem justificada apenas para produção de gluons na região central de rapidez em colisões $p A$.

Por outro lado, no caso de colisões $p p$ e $A A$ a altas energias, os jatos de gluons na região central de rapidez passam a ser produzidos devido à interação de dois sistemas (partônicos) densos. Nestes casos, espera-se que efeitos que levam à quebra da fatorização existente em colisões de sistemas diluído-denso sejam significantes [57] e incluam correções às fórmulas básicas da fatorização $k_{T}$. Todavia, a magnitude dessas correções ainda é um assunto que está sob intenso debate e sua contribuição no regime cinemático provado no LHC não é bem conhecida. Aliado a isso, o fato de que o formalismo da fatorização $k_{T}$ nos permite obter uma boa descrição dos dados experimentais atuais, sugere que estas correções não devem ser grandes, e que, portanto, este formalismo pode ser considerado uma aproximação razoável para o tratamento da produção de gluons em colisões $p p$ e $A A$ na região central de rapidez.

Portanto, no que se segue, deixe-nos aplicar a fatorização $k_{T}$ à descrição da produção de gluons em colisões $p p, p A$ e $A A$, e vamos agora considerar a produção de um gluon com momento transversal $k_{T}$ e rapidez $y$ em uma colisão entre dois hádrons, $h_{1}$ e $h_{2} \operatorname{com} h_{i}=p$ ou $A$. Tendo em vista que as expressões básicas da fatorização $k_{T}$ já foram apresentadas e discutidas na seção 4.1, aqui vamos fazer uma breve recapitulação dos pontos mais importantes para efeito de comparação com outros resultados apresentados nesta seção.

No formalismo da fatorização $k_{T}$, a seção de choque invariante para o processo de interesse é dada por [74] (vide equação (4.2))

$$
\frac{d^{3} \sigma}{d^{2} k_{T} d y}=\frac{2}{C_{F}} \frac{1}{\boldsymbol{k}^{2}} \int d^{2} q \alpha_{s} \phi_{h_{1}}(\boldsymbol{q}, y) \phi_{h_{2}}(\boldsymbol{k}-\boldsymbol{q}, Y-y)
$$

onde $Y$ é o intervalo total de rapidez da colisão, $C_{F}=\left(N_{c}^{2}-1\right) / 2 N_{c}$ e as variáveis 
em negrito denotam vetores no plano transversal, $\boldsymbol{k}=\left(k^{1}, k^{2}\right)$. Além disso, $\phi_{h_{i}}\left(x_{i}, \boldsymbol{q}\right)$ denota a distribuição de gluons não integrada:

$$
\phi_{h_{i}}(\boldsymbol{k}, y)=\frac{C_{F}}{\alpha_{s}(2 \pi)^{3}} \int d^{2} b d^{2} r e^{-i \boldsymbol{k} \cdot \boldsymbol{r}} \nabla_{r}^{2} \mathcal{N}_{h_{i}}^{G}(\boldsymbol{r}, \boldsymbol{b}, y)
$$

onde $\mathcal{N}_{h_{i}}^{G}(\boldsymbol{r}, \boldsymbol{b}, y)$ representa a amplitude de espalhamento frontal dipolo-hádron $h_{i}$ para um dipolo de tamanho transversal $\boldsymbol{r}$ formado por gluons, $\boldsymbol{b}$ representa o parâmetro de impacto do espalhamento.

Embora existam avanços no que se refere à inclusão do parâmetro de impacto na equação BK [410-414], uma das equações que fornecem a evolução de $\mathcal{N}_{h_{i}}^{G}(\boldsymbol{r}, \boldsymbol{b}, y)$ (e portanto de $\left.\phi_{h_{i}}(\boldsymbol{k}, y)\right)$ com a rapidez no formalismo do CGC, a presença de divergências devido à integração em $d^{2} b$ na equação acima é um problema conhecido $[189,190]$ e não solucionado até o momento. Desta maneira, a dependência em $\boldsymbol{b}$ de $\mathcal{N}_{h_{i}}^{G}(\boldsymbol{r}, \boldsymbol{b}, y)$ é, em geral, incluída através de um "ansatz" (geralmente do tipo gaussiano) nos modelos de dipolo fenomenológicos baseados na teoria do CGC e que possuem os parâmetros fixados pelos dados experimentais [134, 135, 164, 166, 296, 395, 406, 407, 409].

$\mathrm{Na}$ fatorização $k_{T}$ a seção de choque invariante, equação (4.20), é proporcional à constante de acoplamento, $\alpha_{s}$, que foi tomada como fixa em sua dreviação. Além disso, $\alpha_{s}$ também aparece na distribuição não integrada de gluons. Correções em $\alpha_{s}$ foram calculadas para a hierarquia de equações BK-JIMWLK nos últimos anos e este fato possibilitou estimar a contribuição dessas correções em $\mathcal{N}_{h_{i}}^{G}(\boldsymbol{r}, \boldsymbol{b}, y)$. No entanto, ainda não é claro como determinar a escala de momento que figura em $\alpha_{s}$ na equação (4.20).

Este fato motivou a generalização ("ingênua") das equações (4.19) e (4.20) pela inclusão de correções na constante de acoplamento [134, 135, 164, 166, 296, 395, 406, 407,409]. Em geral, os estudos citados assumem que a fatorização presente em (4.19) é preservada após a inclusão dessas correções e que a constante de acoplamento nas equações (4.19) e (4.20) dependem de escalas de momento diferentes.

A escolha dessas escalas é arbitrária e existem diferentes escolhas possíveis na literatura. Por exemplo, em $[134,296,409]$ os autores assumiram que $\alpha_{s}=\alpha_{s}\left(k_{T}^{2}\right)$ 
na equação (4.19) e $\alpha_{s}=\alpha_{s}\left(Q_{s}^{2}\left(x_{i}\right)\right)$ na equação (4.20). Por outro lado, em [166] foi assumido que $\alpha_{s}=\alpha_{s}\left(Q^{2}\right)$ na equação (4.19), $\operatorname{com} Q^{2}=\max \left\{k^{2},(k-q)^{2}\right\}$ e a escala que figura em $\alpha_{s}$ na equação (4.20) foi tomada como sendo igual ao momento transversal do gluon.

Uma característica comum dessas abordagens é que elas assumem, em conjunto com a equação (4.19), a expressão em ordem dominante para a constante de acoplamento,

$$
\alpha_{s}\left(Q^{2}\right)=\frac{12 \pi}{\beta_{0} \ln \left(Q^{2} / \Lambda_{Q C D}^{2}\right)} ; \quad \beta_{0}=33-2 n_{f}
$$

onde $\Lambda_{Q C D}$ é uma escala não perturbativa e $n_{f}$ é o número de férmions, como apresentado na seção 1.2.1.

Como $\alpha_{s}\left(Q^{2}\right)$ diverge para pequenos valores de $Q^{2}$, muitas vezes é assumido um congelamento suave do acoplamento nesta região. Por exemplo, em [134, 296,409] o acoplamento forte é fixado em $\alpha_{s}\left(Q^{2}\right)=0.5$ quando $Q^{2} \leq 0.8 \mathrm{GeV}^{2}$.

Como apontado nas referências [134,135,164,166,296,395,406,407,409], a inclusão das correções em $\alpha_{s}$, mesmo que dessa maneira ingênua, melhora a descrição dos dados experimentais. No entanto, como discutido em detalhes em [76], não é claro que a equação (4.19) manterá a sua forma depois da inclusão formal dessas correções. Visando esclarecer este ponto, os autores de [76] estimaram as correções em $\alpha_{s}$ na seção de choque de produção de gluons, derivada em ordem mais baixa da teoria perturbativa (equação (4.19)), encontrando que os três fatores de $\alpha_{s}$ que figuram na expressão não corrigida devem ser substituídos por sete fatores de $\alpha_{s}$. Dois dos acoplamentos possuem escalas de momento com componente imaginária, porém a estrutura do resultado obtido implica em uma seção de choque real. Além disso, a seção de choque é simétrica com respeito à troca das escalas.

Como o cálculo da inclusão dessas correções foi feita apenas para $y=0$ devido à dificuldade envolvida, os autores de [76] também conjecturaram uma generalização da expressão para a seção de choque de produção de gluons em mais baixa ordem, de modo a incluir estas correções de mais alta ordem e também a evolução com a energia 
(ou rapidez):

$\frac{d^{3} \sigma}{d^{2} k_{T} d y}=\frac{2 C_{F}}{\pi^{2}} \frac{1}{\boldsymbol{k}^{2}} \int d^{2} q \bar{\phi}_{h_{1}}(\boldsymbol{q}, y) \bar{\phi}_{h_{2}}(\boldsymbol{k}-\boldsymbol{q}, Y-y) \frac{\alpha_{s}\left(\Lambda_{\mathrm{coll}}^{2} e^{-5 / 3}\right)}{\alpha_{s}\left(Q^{2} e^{-5 / 3}\right) \alpha_{s}\left(Q^{* 2} e^{-5 / 3}\right)}$,

com as distribuições não integradas de gluons definidas por

$$
\bar{\phi}_{h_{i}}(\boldsymbol{k}, y)=\alpha_{s} \phi_{h_{i}}(\boldsymbol{k}, y)=\frac{C_{F}}{(2 \pi)^{3}} \int d^{2} b d^{2} r e^{-i \boldsymbol{k} \cdot \boldsymbol{r}} \nabla_{r}^{2} \mathcal{N}_{h_{i}}(\boldsymbol{r}, \boldsymbol{b}, y)
$$

onde $\Lambda_{\text {coll }}^{2}$ é um corte na região de pequenos momentos transversais e a escala de momento $Q$ é dada por

$$
\begin{aligned}
\ln \frac{Q^{2}}{\mu_{\mathrm{MS}}^{2}} & =\frac{1}{2} \ln \frac{\boldsymbol{q}^{2}(\boldsymbol{k}-\boldsymbol{q})^{2}}{\mu_{\mathrm{MS}}^{4}}-\frac{1}{4 \boldsymbol{q}^{2}(\boldsymbol{k}-\boldsymbol{q})^{2}\left[(\boldsymbol{k}-\boldsymbol{q})^{2}-\boldsymbol{q}^{2}\right]^{6}}\left\{\boldsymbol{k}^{2}\left[(\boldsymbol{k}-\boldsymbol{q})^{2}-\boldsymbol{q}^{2}\right]^{3}\right. \\
& \times\left\{\left[(\boldsymbol{k}-\boldsymbol{q})^{2}\right]^{2}-\left(\boldsymbol{q}^{2}\right)^{2}\right]\left[\left(\boldsymbol{k}^{2}\right)^{2}+\left((\boldsymbol{k}-\boldsymbol{q})^{2}-\boldsymbol{q}^{2}\right)^{2}\right]+2 \boldsymbol{k}^{2}\left[\left(\boldsymbol{q}^{2}\right)^{3}-\left[(\boldsymbol{k}-\boldsymbol{q})^{2}\right]^{3}\right] \\
& \left.-\boldsymbol{q}^{2}(\boldsymbol{k}-\boldsymbol{q})^{2}\left[2\left(\boldsymbol{k}^{2}\right)^{2}+3\left[(\boldsymbol{k}-\boldsymbol{q})^{2}-\boldsymbol{q}^{2}\right]^{2}-3 \boldsymbol{k}^{2}\left[(\boldsymbol{k}-\boldsymbol{q})^{2}+\boldsymbol{q}^{2}\right]\right] \ln \left(\frac{(\boldsymbol{k}-\boldsymbol{q})^{2}}{\boldsymbol{q}^{2}}\right)\right\} \\
& +i\left[(\boldsymbol{k}-\boldsymbol{q})^{2}-\boldsymbol{q}^{2}\right]^{3}\left\{\boldsymbol{k}^{2}\left[(\boldsymbol{k}-\boldsymbol{q})^{2}-\boldsymbol{q}^{2}\right]\left[\boldsymbol{k}^{2}\left[(\boldsymbol{k}-\boldsymbol{q})^{2}+\boldsymbol{q}^{2}\right]-\left(\boldsymbol{q}^{2}\right)^{2}-\left[(\boldsymbol{k}-\boldsymbol{q})^{2}\right]^{2}\right]\right. \\
& \left.+\boldsymbol{q}^{2}(\boldsymbol{k}-\boldsymbol{q})^{2}\left(\boldsymbol{k}^{2}\left[(\boldsymbol{k}-\boldsymbol{q})^{2}+\boldsymbol{q}^{2}\right]-2\left(\boldsymbol{k}^{2}\right)^{2}-2\left[(\boldsymbol{k}-\boldsymbol{q})^{2}-\boldsymbol{q}^{2}\right]^{2}\right) \ln \left(\frac{(\boldsymbol{k}-\boldsymbol{q})^{2}}{\boldsymbol{q}}\right)\right\} \\
& \left.\times \sqrt{2 \boldsymbol{q}^{2}(\boldsymbol{k}-\boldsymbol{q})^{2}+2 \boldsymbol{k}^{2}(\boldsymbol{k}-\boldsymbol{q})^{2}+2 \boldsymbol{q}^{2} \boldsymbol{k}^{2}-\left(\boldsymbol{k}^{2}\right)^{2}-\left(\boldsymbol{q}^{2}\right)^{2}-\left[(\boldsymbol{k}-\boldsymbol{q})^{2}\right]^{2}}\right\} .
\end{aligned}
$$

Como é possível notar, diferentemente da equação (4.19), na expressão corrigida (equação (4.22)), todas as escalas de momento que figuram nas constantes de acoplamento são especificadas via generalização de um resultado formal. Em [76] os autores reivindicam que esta equação, assim como a equação (4.19), deve ser válida nos regimes linear e não linear da QCD. Porém, como enfatizado neste mesmo trabalho, apenas cálculos exatos são capazes de verificar a validade desta conjectura.

Espera-se que a equação (4.22) seja uma boa aproximação para a resposta exata, 
uma vez que generaliza um resultado exato, porém obtido para rapidez fixa. Esta expectativa motiva um estudo fenomenológico utilizando a fórmula da fatorização $k_{T}$ com correções na constante de acoplamento. Na próxima seção apresentamos os ingredientes utilizados neste estudo fenomenológico bem como os resultados obtidos.

\subsubsection{Resultados}

Nesta seção as predições da fórmula da fatorização $k_{T}$ com correções na constante de acoplamento, dada pela equação (4.22) e denotada por CF daqui em diante, são comparadas com aquelas obtidas através da fórmula original, equação (4.19). Neste último caso a seção de choque inclusiva para produção de gluons foi calculada assumindo-se um valor fixo para constante de acoplamento (os resultados obtidos por meio desta escolha são denotados por FC) e também assumindo-se que os acoplamentos são dados pela equação (4.21) com as escalas $Q^{2}$ seguindo a prescrição das referências [166] e $[134,296,409]$. Os resultados obtidos com esta configuração são denotados por RC1 e $\mathrm{RC} 2$, respectivamente.

De modo a estudar o impacto das correções em $\alpha_{s}$ na fórmula da fatorização $k_{T}$ as seguintes aproximações foram tomadas: $i$ ) toda e qualquer dependência da distribuição não integrada de gluons com o parâmetro de impacto foi ignorada e considerou-se apenas colisões do tipo "minimum bias", onde, como na seção 4.4, assumiu-se que $A_{\text {eff }}=20(18.5)$ para $\mathrm{Pb}(\mathrm{Au})$; ii) assumiu-se a validade da chamada "Local PartonHadron Duality" [415-417], significando que a forma de uma dada distribuição (de rapidez, por exemplo) para hádrons difere da mesma distribuição a nível partônico (isto é, para gluons) apenas por um fator constante. Esse procedimento introduz uma massa efetiva, $m_{h}$ (fixada em $0,350 \mathrm{GeV}$ ) que incorpora efeitos não perturbativos e iii) apenas um modelo para a distribuição não integrada de gluons foi, o modelo KLN [38-40], que incorpora os aspectos básicos da dinâmica da QCD no regime não 
linear (ou de saturação), dado por,

$$
\begin{aligned}
\phi_{K L N}(\boldsymbol{k}, y) & =\frac{2 C_{F}}{3 \pi^{2} \alpha_{s}}, \quad k \leq Q_{s} \\
& =\frac{2 C_{F}}{3 \pi^{2} \alpha_{s}} \frac{Q_{s}^{2}}{k^{2}}, \quad k>Q_{s},
\end{aligned}
$$

onde a escala de saturação é dada por $Q_{s}^{2}=A_{\text {eff }}^{1 / 3} Q_{0}^{2}\left(x_{0} / x\right)^{\lambda}$, com $Q_{0}=1 \mathrm{GeV}$, $x_{0}=3 \times 10^{-4}$ e $\lambda=0.288$ [113]. Como comumente utilizado na literatura [38-40,134, 296, 409], multiplicamos a distribuição $\phi_{K L N}$ pelo fator $(1-x)^{4}$ de modo a simular o comportamento dessa distribuição na região de grandes valores de $x_{1,2}(\rightarrow 1)$ como prescrito pelas regras de contagem de quarks [418,419].

Embora as aproximações aqui utilizadas possam e devam ser melhoradas em um calculo quantitativo dos observáveis análisados nesta seção, acreditamos que nossa análise simplificada possa fornecer uma primeira indicação de como as correções na constante de acoplamento (incluídas na fórmula da fatorização $k_{T}$ ) influenciam esses observáveis.

A seção de choque para a produção inclusiva de hádrons nessa abordagem é dada por

$$
\frac{d^{3} N}{d \eta d^{2} k_{T}}=\frac{K}{\sigma_{s}} h\left(y, k_{T}, m_{h}\right) \cdot \frac{d^{3} \sigma}{d^{2} k_{T} d y},
$$

onde $\eta$ representa a pseudorapidez, $h\left(y, k_{T}, m_{h}\right)$ representa o jacobiano da transformação da variável rapidez para pseudorapidez, dado por

$$
h\left(y, k_{T}, m_{h}\right)=\sqrt{1-\frac{m_{h}^{2}}{m_{T}^{2} \cosh ^{2} y}},
$$

onde $m_{h}$ representa a massa do hádron produzido na colisão, $m_{T}$, definido pela relação $m_{T}^{2}=k_{T}^{2}+m_{h}^{2}$, representa sua massa transversal e $\sigma_{s}$ representa a área de interação média dos hádrons colidentes.

Como em outras seções, o fator $K$ que figura nessa equação, incorpora (de uma maneira efetiva) correções de mais alta ordem e também efeitos que não estão incluídos 
no formalismo do CGC, assim como incertezas na conversão dos pártons em hádrons. Da mesma forma que em trabalhos anteriores [134, 296, 409], a substituição $k_{T} \rightarrow$ $\sqrt{k_{T}^{2}+m_{h}^{2}}$ foi efetuada na definição da vairável de Bjorken, $x_{1,2}\left(k_{T} e^{ \pm y} / \sqrt{s}\right)$ e no fator $1 / \boldsymbol{k}^{2}$ que figura nas equações (4.19) e (4.22), de modo a corrigir os elementos cinemáticos dos cálculos devido à presença de uma escala de massa. Além disso, escolheu-se $\alpha_{s}=0.25$ nos resultados obtidos com a constante de acoplamento fixa, enquanto que as predições denotadas por RC1 e RC2, por outro lado, foram obtidas fixando-se $N_{c}=3, n_{f}=3$ e $\Lambda_{Q C D}=0.240 \mathrm{GeV}$ na equação (4.21). No caso da expressão corrigida em $\alpha_{s}$ assumiu-se $\alpha_{s}\left(\Lambda_{\text {coll }}^{2} e^{-5 / 3}\right)=0.25$ e o valor de $\mu_{\overline{\mathrm{MS}}}$ foi fixado exigindo-se que $\mu_{\overline{\mathrm{MS}}}^{2} e^{5 / 3}=\Lambda_{Q C D}^{2}$. Finalmente, o fator de normalização $K / \sigma_{s}$ foi tratado como um parâmetro livre, a ser fixado pela comparação com os dados experimentais para um dado valor de energia e rapidez.

A Figura 4.27 apresenta os resultados obtidos para a distribuição em pseudorapidez em colisões $p p$ para diferentes energias do centro de massa. A normalização das diferentes curvas, dada pelo fator $K / \sigma_{s}$ na equação (4.26), foi fixada para cada valor de energia de modo a reproduzir os dados experimentais em $\eta=0$. As predições FC e RC1 são muito parecidas e diferem dos outros resultados na região de grande valores de $\eta$; por outro lado, o resultado RC2 exibe um aumento (e queda) mais acentuado com a pseudorapidez. A curva CF produz uma descrição razoável dos dados experimentais em colisões $p p$. Enquanto que, nesse caso, a mudança necessária na normalização dessas predições entre as energias $\sqrt{s}=0.9 \mathrm{TeV}$ e $7 \mathrm{TeV}$ foi menor que $1 \%$, ela foi maior que $19 \%$ para todas as outras predições.

Na Figura 4.28 são apresentados os resultados para a distribuição em pseudorapidez para a produção inclusiva de hádrons em colisões $d A u$, onde a energia de colisão é $\sqrt{s}=0.2 \mathrm{TeV}$, e em colisões $p P b$ onde a energia de colisão é $\sqrt{s}=5.02 \mathrm{TeV}$. A normalização dos resultados para colisões $d A u$ foi escolhida de modo a descrever simultaneamente os dados experimentais na direção do dêuteron (isto é, a região de pseudorapidez negativa) e a região de $\eta=0$. Já para as colisões $p P b$, as curvas foram normalizadas de modo a descrever os dados experimentais na região de $\eta=0$. Os 


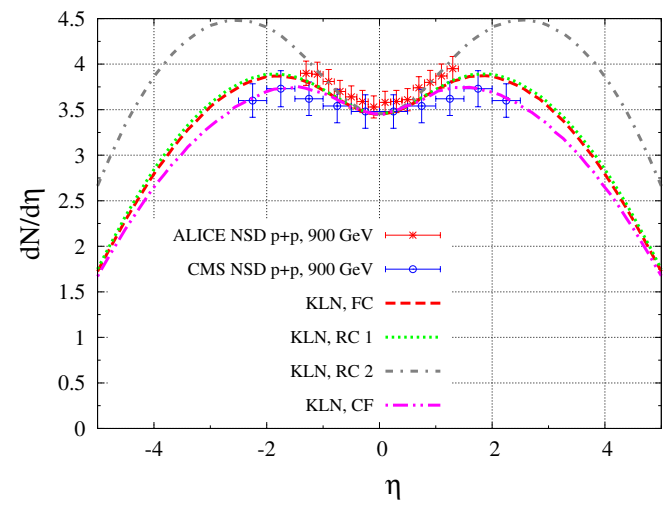

(a)

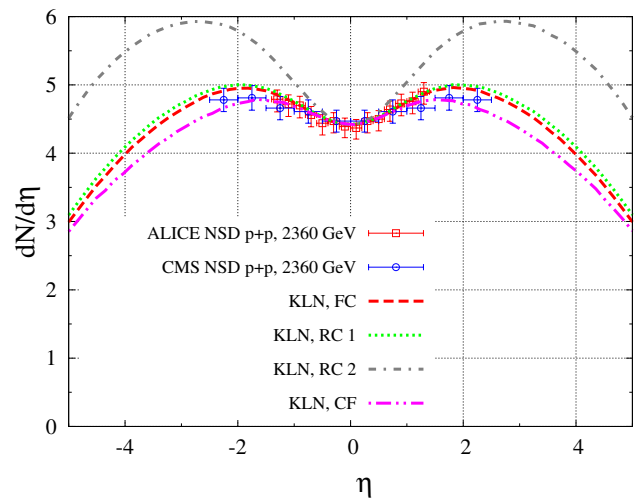

(b)

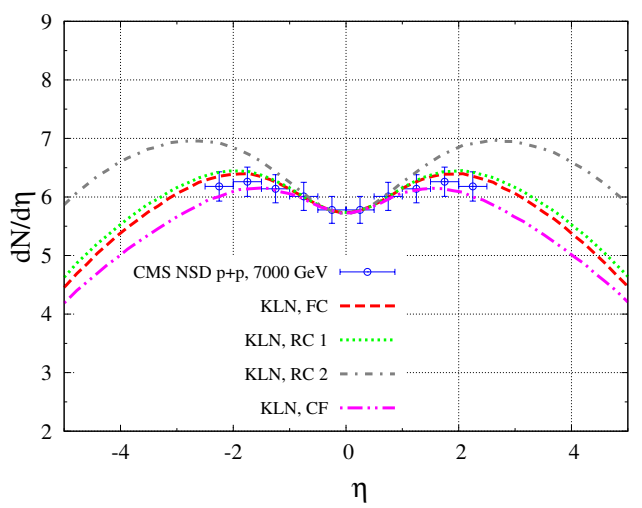

(c)

Figura 4.27: Distribuições de pseudorapidez para a produção inclusiva de hádrons em colisões pp para diferentes valores de $\sqrt{s}$ : (a) $0,9 \mathbf{T e V}$, (b) 2,36 $\mathrm{TeV}$ e (c) $7 \mathrm{TeV}$. Os dados experimentais são das referências [388, 389, 420].

resultados obtidos para essas distribuições nesses casos repetem os comportamentos dos resultados obtidos para as mesmas distribuições em colisões $p p$, apresentadas na Figura 4.27 e, portanto, conduzem à uma análise equivalente a já apresentada acima. A discrepância existente para grandes valores de $\eta$ em colisões $d A u$, por outro lado, pode ser atribuído ao tratamento simplificado para a geometria nuclear considerado nos nossos cálculos.

A normalização $K / \sigma_{s}$ necessária para descrever esses dados experimentais nas diferentes energias consideradas é de cerca de $17 \%$ para as predições CF enquanto a mudança para as outras predições é da ordem de 40\%. Uma possível interpretação para este fato é que a fórmula corrigida captura importantes correções de mais altas or- 


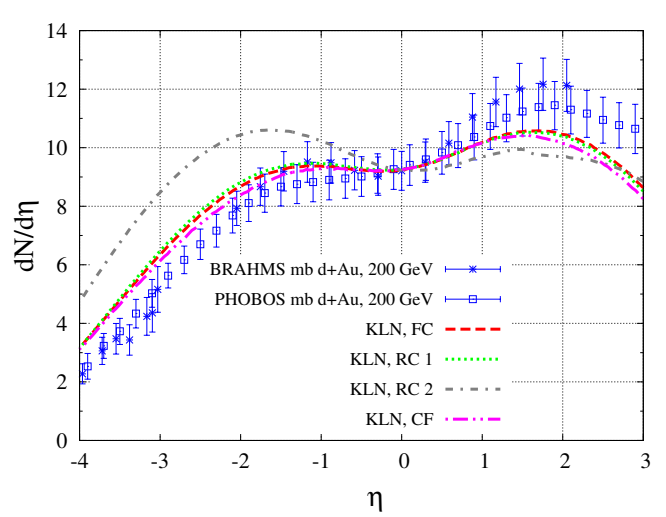

(a)

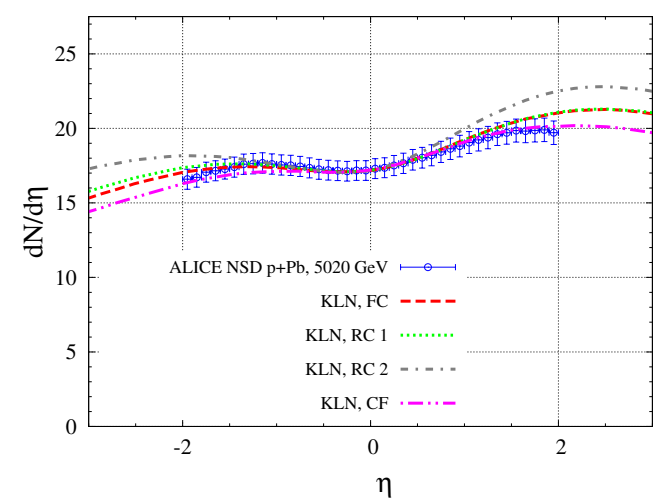

(b)

Figura 4.28: Distribuições de pseudorapidez para a produção inclusiva de hádrons em (a) colisões $d A u(\sqrt{s}=0.2 \mathrm{TeV})$ e (b) colisões $p P b(\sqrt{s}=5.02 \mathrm{TeV})$. Os dados experimentais são das referências [421-423].

dens que são dependentes da energia, uma vez que o mesmo modelo para a distribuição de gluons não integrada foi utilizado em todas as predições.

Os resultados para a distribuição de rapidez em colisões $A u A u$ em $0.2 \mathrm{TeV}$ e colisões $\mathrm{PbPb}$ em $2.76 \mathrm{TeV}$ são apresentados na Figura 4.29 Novamente, a normalização das diferentes curvas foi fixada de modo que os dados na região de $\eta=0$ fossem reproduzidos. Neste caso, a dependência das diferentes curvas é semelhante, com o resultado $\mathrm{CF}$ fornecendo uma descrição mais razoável dos dados experimentais. Em contraste com os casos anteriores (colisões $p p$ e $d A u / p P b$ ) em colisões de íons pesados a mudança necessária na normalização, indo da energia mais baixa para a mais alta, é sempre maior, mesmo para o resultado $\mathrm{CF}[\mathcal{O}(25 \%)]$. Este fato pode ser um indício de que um tratamento mais cuidadoso da geometria nuclear deve ser considerado em colisões de íons pesados.

A predição das diferentes fórmulas da fatorização $k_{T}$ para a multiplicidade de hádrons carregados na região central de pseudorapidez em função da energia da colisão $(p p, p A$ e $A A)$ é agora analisada. As predições em colisões $p A$ e $A A$ foram normalizadas a $\left\langle N_{\text {part }}\right\rangle$ e $2 /\left\langle N_{\text {part }}\right\rangle$, respectivamente, onde $\left\langle N_{\text {part }}\right\rangle$ representa o número médio de participantes numa colisão. Os valores de $\left\langle N_{\text {part }}\right\rangle$ em colisões minimum bias $p P b$ e em 3\% das colisões $A A$ mais centrais, apresentados nas referências [423,428], foram 


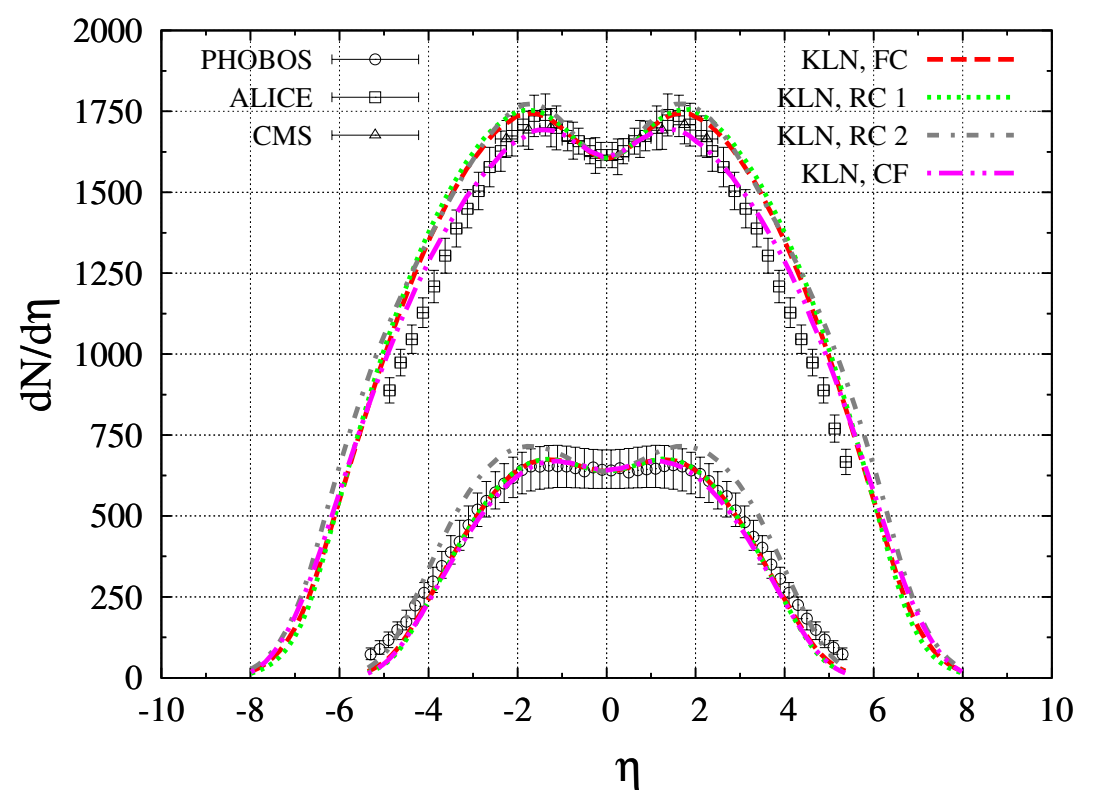

Figura 4.29: Distribuições de pseudorapidez para a produção inclusiva de hádrons em colisões $A u A u$ em $\sqrt{s}=0.2 \mathrm{TeV}$ (curvas inferiores) e em colisões $\mathrm{PbPb}$ em $\sqrt{s}=2.76 \mathrm{TeV}$ (curvas superiores). Os dados experimentais são das referências [424-427].

utilizados.

A normalização utilizada nesses resultados foi a mesma utilizada nas distribuições de $d N / d \eta$ apresentados anteriormente em colisões $p p$ a $\sqrt{s}=0.9 \mathrm{TeV}$, colisões $d A u$ a $\sqrt{s}=0.2 \mathrm{TeV}$ e colisões $A u A u$ a $\sqrt{s}=0.13 \mathrm{TeV}$. Com esse expediente, as predições para energias maiores tornam-se independentes de ajustes de parâmetros.

Como pode ser visto na Figura 4.30, a curva $\mathrm{CF}$ apresenta um crescimento mais lento com a energia em comparação com as predições obtidas via fórmula original da fatorização $k_{T}$. Chama a atenção que, mesmo com o uso de um modelo simplificado para a distribuição não integrada de gluons e um tratamento não muito refinado para a geometria nuclear, a fórmula da fatorização $k_{T}$ com correções na constante de acoplamento fornece uma descrição satisfatória dos dados experimentais em colisões $p p$ e $p A$. Em particular, a predição CF descreve bem os dados experimentais em colisões $p p$ na região de baixas e altas energias quando contrastada com outras abordagens que, utilizando os mesmos ingredientes, falham em reproduzir os dados experimentais para $\sqrt{s}<0.9 \mathrm{TeV}$. Além disso, a fórmula corrigida fornece uma predição que está 


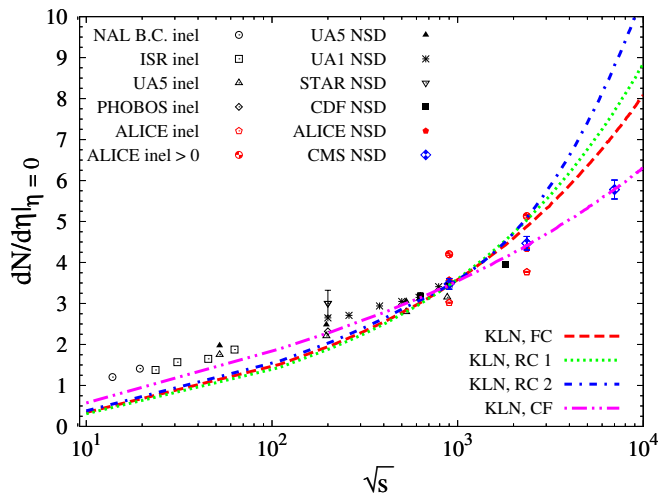

(a)

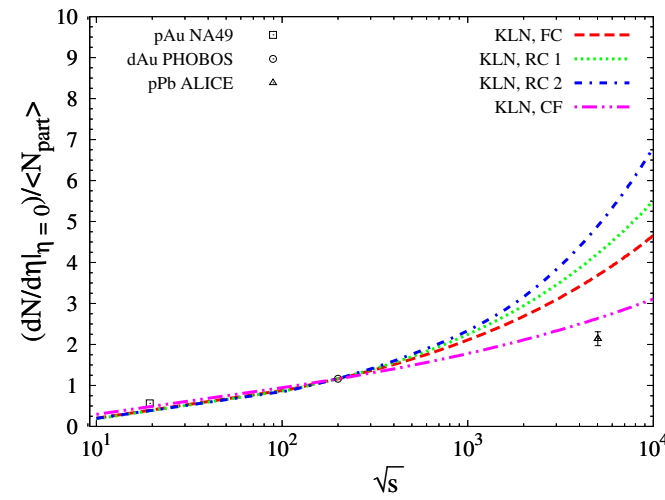

(b)

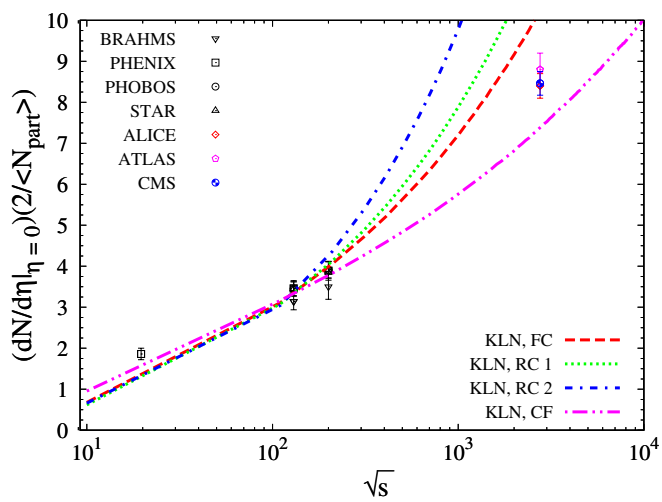

(c)

Figura 4.30: Dependência energética da multiplicidade de hádrons na região central de pseudorapidez para colisões (a) pp, (b) $p A$ e (c) $A A$. Os resultados para colisões $p A$ e $A A$ são normalizados por $N_{\text {part }}$ e $2 /\left\langle N_{\text {part }}\right\rangle$, respectivamente. Os dados experimentais são das referências [136, 388, 420, 423, 427-440].

mais próxima dos dados experimentais no caso de colisões $p A$. Para colisões $A A$, a predição CF subestima os dados experimentais na região de altas energias, fato que pode indicar que um tratamento mais preciso da distribuição não integrada de gluons e da geometria nuclear seja fundamental para uma melhor descrição desses dados. 


\section{Capítulo 5}

\section{Assimetrias angulares em colisões próton-núcleo a altas energias}

Neste capítulo estudamos as assimetrias angulares observadas em colisões $p A$ no LHC. Usualmente tais assimetrias são descritas fazendo-se uso de simulações hidrodinâmicas, onde a anisotropia do sistema formado é gerada no estado final da colisão, conforme discutido brevemente no capítulo 2. Aqui, no entanto, apresentamos um estudo de cunho exploratório acerca do surgimento de assimetrias angulares através da anisotropia gerada no estado inicial da colisão $p A$ devido a flutuação das cargas de cor no núcleo alvo. No que se segue, apresentamos as ideias que fundamentam o modelo proposto, os ingredientes utilizados em sua construção, bem como os resultados obtidos.

\subsection{Correlação de partículas}

Colisões $p A$ e $A A$ a altas energias, como as que ocorrem no RHIC e no LHC, têm como resultado a produção de um número muito grande de partículas. Uma vez que apenas bárions e mésons são observados experimentalmente, devido à propriedade 
do confinamento da QCD, faz-se necessário encontrar observáveis físicos que sejam sensíveis à dinâmica partônica do processo colisional que carreguem informações sobre os estágios iniciais da colisão, onde espera-se a formação de um meio extremamente denso e colorido no qual importantes efeitos nucleares encontram-se presentes.

Em [178] foi proposto que a presencça de anisotropias na distribuição de momento transversal das partículas produzidas em colisões núcleo-núcleo poderiam fornecer assinaturas não ambíguas do fluxo coletivo dessas partículas e, portanto, seriam sensíveis às informações sobre a evolução do sistema partônico criado nos estágios iniciais da colisão. A nível experimental, medidas dos chamados "harmônicos azimutais" em colisões hadrônicas permitem investigar a presença ou não de anisotropias na distribuição acima mencionada com relação ao chamado "plano de reação", $\Psi^{R P}$. Na abordagem usual $[149,178]$, o plano de reação é formado pelo parâmetro de impacto $(\vec{b})$, que aponta para o centro dos dois núcleos colidentes e a direção de propagação dos feixes de partículas colidentes, z. É importante mencionar que a existência de um plano de reação leva à quebra espontânea da simetria rotacional do sistema, justamente por definir uma direção preferencial para sua evolução após a colisão.

Em uma colisão $A A$ não central, isto é, com um parâmetro de impacto não nulo e finito, a geometria inicial da colisão é anisotrópica com relação ao plano de reação na direção azimutal, como mostra a Figura 5.1. A maneira usual de se caracterizar os vários padrões de anisotropia é realizar uma expansão em série de Fourier da seção de choque invariante com respeito ao ângulo do plano de reação [441,442],

$$
E \frac{d^{3} N}{d p^{3}}=\frac{d^{3} N}{p_{T} d p_{T} d \phi_{p} d y}\left(1+2 \sum_{n=1}^{\infty} v_{n}\left(p_{T}, \phi\right) \cos \left[n\left(\phi-\Psi_{n}^{R P}\right)\right]\right)
$$

onde $E, p_{T}, \phi_{p}, y$ e $\Psi_{n}^{R P}$ representam, respectivamente, a energia do processo, o momento transversal, o ângulo azimutal, a rapidez das partículas produzidas e o ângulo do plano de reação do $n$-ésimo harmônico azimutal.

Nessa expressão, ambos os ângulos, $\phi$ e $\Psi_{n}^{R P}$, são medidos no referencial do laboratório. Os coeficientes $v_{n}\left(p_{T}, \phi\right)$ são justamente os harmônicos azimutais mencionados 
acima, dados por:

$$
v_{n}\left(p_{T}, \phi\right) \equiv\left\langle e^{i n\left(\phi-\Psi_{n}^{R P}\right)}\right\rangle=\left\langle\cos \left[n\left(\phi-\Psi_{n}^{R P}\right)\right]\right\rangle
$$

onde " \langle\rangle " denota uma média sobre todas as partículas de um evento, com a posterior média tomada sobre todos os eventos ${ }^{1}$.

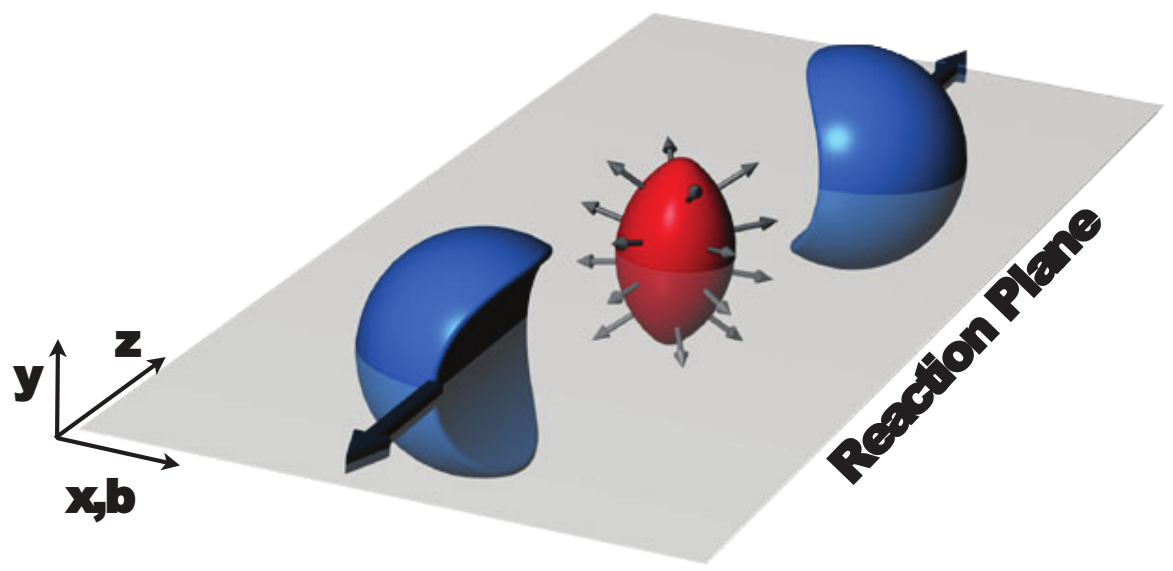

Figura 5.1: Esquema tridimensional de uma colisão AA não central. $O$ volume em forma de amêndoa representa as partículas participantes na colisão; na forma em azul encontram-se os nucleons que não participam da colisão ("espectadores") e seguem em sentidos opostos. O plano de reação é formado por $x$, que esta orientado na direção do parâmetro de impacto $(\vec{b})$ e $z$, a direção de propagação do feixe. Extraído de [149].

Uma vez que o ângulo do plano de reação, $\Psi_{n}^{R P}$, não pode ser observado diretamente, tem-se que os coeficientes $v_{n}\left(p_{T}, \phi\right)$ também não podem ser medidos diretamente. Entretanto, $\Psi_{n}^{R P}$ pode ser estimado a partir da distribuição azimutal das partículas produzidas evento a evento [150] e, portanto, os diferentes harmônicos azimutais podem ser reconstruídos a partir de correlações angulares entre duas ou mais partículas.

Por simplicidade, o n-ésimo harmônico azimutal devido à correlação de duas partículas

\footnotetext{
${ }^{1} \mathrm{Na}$ equação $(5.2)$, os termos proporcionais a $\sin \left[n\left(\phi-\Psi_{n}^{R P}\right)\right]$ se anulam quando assumido que a produção de partículas é simétrica com relação ao plano de reação.
} 
é dado por [443]

$$
\left(v_{n}\{2\}\right)^{2}=\left\langle e^{n i\left(\left(\phi_{1}-\Psi_{n}^{R P}\right)-\left(\phi_{2}-\Psi_{n}^{R P}\right)\right)}\right\rangle=\left\langle e^{n i\left(\phi_{1}-\phi_{2}\right)}\right\rangle
$$

onde $\phi_{j}$ é o ângulo azimutal da $j$-ésima partícula.

É importante notar que este observável é sempre definido de modo a ser invariante por uma rotação global, $\phi_{i} \rightarrow \phi_{i}+\varphi$ e, por conta disso, a dependência com o ângulo do plano de reação desaparece.

O primeiro cálculo do "momento de quadrupolo", $v_{2} \equiv\langle\cos (2 \phi)\rangle$, gerado no estado inicial de colisões hadrônicas a altas energias, pode ser encontrado em [444], onde um $v_{2}$ não nulo emerge do fato de que a seção de choque para a produção de dois gluons é favorecida a um ângulo relativo $\phi_{1}-\phi_{2} \sim[0, \pi]$ porém é suprimida para $\phi_{1}-\phi_{2} \sim \pm \pi / 2$.

Por envolver a correlação de apenas duas partículas, o valor de $v_{2}\{2\}$ não é constante. Na verdade, seu valor flutua evento a evento devido à presença de outras fontes de correlações entre duas partículas produzidas após a colisão [153, 154, 179-182]. A emissão de jatos, decaimentos em ressonâncias e a conservação de momento das partículas produzidas são exemplos de outras fontes de correlação. Uma vez que correlações desse tipo ocorrem de maneira independente do plano de reação, elas são classificadas como correlações do tipo "não fluxo" (non-flow). Estudos experimentais realizados com dados do RHIC [445] mostraram que as correlações do tipo non-flow contribuem de forma não desprezível na região de grandes momentos e afetam principalmente a correlação de duas partículas.

Todavia, a natureza coletiva das correlações entre as parículas produzidas em um dado evento pode ser utilizada para suprimir a contribuição de correlações do tipo nonflow. Isto é feito estudando-se a correlação entre um número maior de partículas, os chamados "cumulantes de mais alta ordem" [443]. Por exemplo, o segundo harmônico obtido a partir da correlação entre dois pares de partículas, $v_{2}\{4\}$, é dado por [443]

$$
\left(v_{2}\{4\}\right)^{4}=2\left\langle e^{2 i\left(\phi_{1}-\phi_{3}\right)}\right\rangle\left\langle e^{2 i\left(\phi_{2}-\phi_{4}\right)}\right\rangle-\left\langle e^{2 i\left(\phi_{1}+\phi_{2}-\phi_{3}-\phi_{4}\right)}\right\rangle .
$$


É importante mencionar que o fato de se ter $v_{2}\{2\} \neq 0$ e $v_{2}\{4\} \neq 0$ não implica que exista uma quebra espontânea da simetria rotacional devido à correlação destas partículas com um plano de reação. Para um número finito de partículas, as correlações entre múltiplas partículas que contribuem para $v_{2}$ também podem emergir de flutuações [446-448].

A despeito disso, no estudo que aqui apresentamos, tivemos cuidado em verificar se a dependência com $p_{T}$ de $v_{2}$ é compatível com a quebra espontânea da simetria rotacional devido à formação de um condensado do campo elétrico de cor $(\vec{E})$ no núcleo alvo [170], que implica em $v_{2 n} \neq 0$, como será mostrado a seguir.

Em [170], Kovner e Lublinsky sugeriram a formação de condensados de campos elétricos de cores com comprimento de correlação dado pela escala de saturação $(\lambda \sim$ $\left.1 / Q_{s}\right)$ em um núcleo grande que se move relativisticamente. Os detalhes da origem de tais condensados ainda estão sob investigação. No entanto, foi suposto que sua formação é devida às flutuações na evolução em pequeno $x$ 's dependente do parâmetro de impacto, de um sistema nuclear que apresenta um comprimento de correlação da ordem de $1 / Q_{s}$. Caso exista um comprimento de correlação da ordem de $1 / Q_{s}$ aparenta ser razoável que, nessa escala de distâncias, o vetor $\vec{E}$ possa apontar em uma direção fixa, porém aleatória, a cada evento.

A Figura 5.2 apresenta um esquema das configurações do campo elétrico de cor típicas em um núcleo; cada região pode ser pensada como um "domínio"de $\vec{E}$. Este é, portanto, um típico efeito de coerência.

Tal condensado formado por $\vec{E}$ quebra espontaneamente a simetria rotacional na direção do feixe. Enquanto que a direção de $\vec{E}$ em um dado evento particular é, com certeza, aleatória, a distribuição azimutal da seção de choque para a produção inclusiva de pártons com relação a essa direção preferencial é não uniforme ${ }^{2}$. O condensado fornece a direção preferencial para qualquer partícula espalhada por um campo elétrico de cor $\vec{E}$ particular.

\footnotetext{
${ }^{2}$ Nesse sentido, o $v_{2}$ gerado em colisões $p A$ a partir de um condensado vetorial é análogo ao $v_{2}$ "padrão" oriundo de colisões $A A$ não centrais, onde a simetria rotacional é espontâneamente quebrada por um parâmetro de impacto, $\vec{b}$, não nulo que aponta em uma direção aleatória.
} 


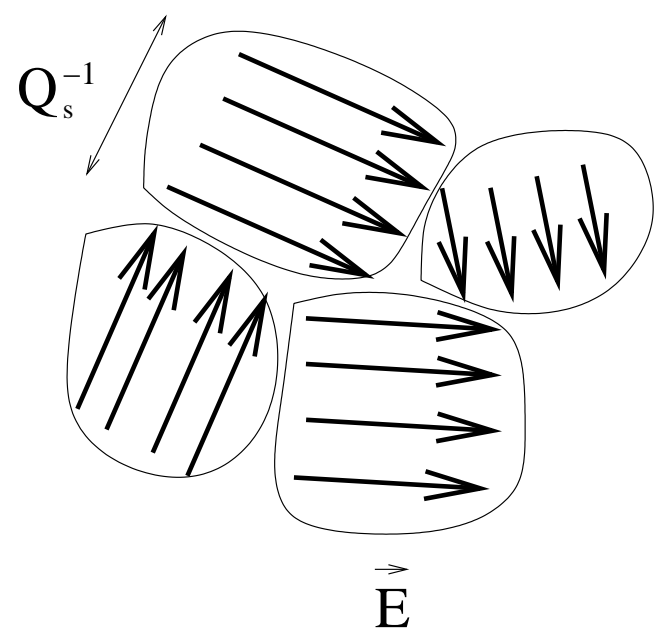

Figura 5.2: Configurações do campo elétrico de cor típicas em um núcleo. Cada região representa um “domínio"diferente de $\vec{E}$. Extraído de [170]

Um dos principais objetivos neste capítulo consiste no cálculo de $v_{2}\left(p_{T}\right)$ e $v_{4}\left(p_{T}\right)$ para uma interação dipolo-campo elétrico de cor, $\vec{r} \cdot \vec{E}$. Embora tenhamos nos focado no cálculo de harmônicos azimutais para uma rapidez fixa, neste caso as correlações se estendem sobre um grande intervalo de rapidez devido à invariância (aproximada) dos campos do núcleo alvo por transformações de Lorentz do tipo "boost" [169]. É importante mencionar que nesses cálculos também não foram incluídas flutuações que contribuem para $v_{2} \neq 0$ (de fato, o resultado obtido para $v_{2 n}$ é nulo sem a presença de um condensado) e, portanto, os resultados aqui apresentados estão mais próximos de serem comparados a $v_{2}\{4\}$ do que a $v_{2}\{2\}^{3}$.

Outro ponto importante é que, em ordem dominante no número de cores, $N_{c}$, nesta abordagem, a seção de choque invariante para a produção de $n$ partículas se fatoriza em $n$ seções de choque invariantes, cada uma contendo as informações específicas de uma dada partícula produzida. Em outras palavras, cada partícula é produzida de maneira independente, com cada uma delas exibindo uma correlação angular com o plano de reação definido por $\vec{E}$ [169]. Os diagramas que consideram a correlação

\footnotetext{
${ }^{3}$ Deve-se enfatizar novamente que, para um número finito de partículas, flutuações podem gerar correlações entre duas ou mais partículas no espaço de coordenadas [446-448] que, por sua vez, podem gerar correlações no espaço dos momentos no estado final. Tais flutuações afetam, predominantemente, $v_{2}\{2\}[445]$.
} 
genuína entre as várias partículas produzidas [140,160-163] são de ordem $\sim 1 / N_{c}^{2} \mathrm{e}$ fornecem correções à fatorização das correlações angulares $[165,176]$.

Harmônicos azimutais ímpares, $v_{2 n+1}$, também podem ser gerados a partir de flutuações com paridade de carga ímpar [378]. Estes momentos emergem de contribuições de paridade ímpar (sob a transformação $\phi \rightarrow \phi+\pi$ ) para a distribuição inclusiva de partículas. No cenário do modelo de dipolo de cor [64-69] estas flutuações estão relacionadas com a parte imaginária da matriz de espalhamento [449], a matriz $S$, exatamente da mesma forma que as assimetrias de spin geradas em colisões de núcleos (alvo) pesados por prótons polarizados $[450,451]$. No estudo que apresentamos, os momentos ímpares, $v_{2 n+1}\left(p_{T}\right)$, foram gerados a partir de flutuações espontâneas na densidade de matéria do núcleo alvo [449].

Outro objetivo do estudo apresentado neste capítulo é fornecer um simples modelo para $v_{1}$ e $v_{3}$ (sem considerar interações de estado final), e extrair uma amplitude e uma escala para as flutuações de paridade de carga ímpar. Como assumido no cálculo dos harmônicos pares, $v_{2 n}$, supõem-se que todos os vetores de onda das flutuações com paridade de carga ímpar apontam na mesma direção e, portanto, fornecem uma direção "global" para os hamônicos $v_{2 n+1}$ que quebra a invariância rotacional espontaneamente.

A seguir apresentamos os ingredientes básicos do modelo utilizado no estudo das assimetrias azimutais, os detalhes de sua formulação e os resultados obtidos.

\subsection{A distribuição de momento de quarks espalha- dos em colisões $p A$}

A exemplo dos capítulos anteriores, a abordagem do CGC para o desenvolvimento dos cálculos a serem apresentados nas próximas seções foi empregada. Mais especificamente, utilizamos o formalismo híbrido, apresentado na seção 4.1 e aplicado em três diferentes estudos, já discutidos no capítulo anterior. 
Neste caso, a distribuição de momento transversal de um quark que sofre um espalhamento elástico com o alvo é dada pela equação (2.26). Quando combinada com a equação (2.10), a equação (2.26) pode ser escrita como,

$$
\begin{aligned}
\frac{d N}{d^{2} k} & =\frac{1}{(2 \pi)^{2}} \int d^{2} r e^{-i \vec{k} \cdot \vec{r}} \frac{1}{N_{c}}\left\langle\operatorname{tr}\left(V^{\dagger}(\vec{b}+\vec{r} / 2) V(\vec{b}-\vec{r} / 2)\right)\right\rangle \\
& =\frac{1}{(2 \pi)^{2}} \int d^{2} r e^{-i \vec{k} \cdot \vec{r}} S(\vec{r}) .
\end{aligned}
$$

Na equação acima, " \langle\rangle " denota que se deve tomar a média sobre todas as configurações de carga de cor do alvo. Para um operador $\mathcal{O}$ qualquer tem-se [35-43]

$$
\langle\mathcal{O}\rangle=\frac{\int D[\rho] \mathcal{O} W[\rho]}{\int D[\rho] W[\rho]}
$$

onde $\rho \equiv \rho^{a}\left(x^{-}, x_{\perp}\right)$ representa a densidade de carga de cor de índice a localizada na coordenada longitudinal $x^{-}$e no plano transversal $x_{\perp}$ do alvo,

$$
D[\rho] \equiv \prod_{a} \prod_{x^{-}} \prod_{x_{\perp}} d \rho^{a}\left(x^{-}, x_{\perp}\right)
$$

e o funcional $W[\rho]$ representam, respectivamente, a medida funcional de todas as configurações de cor do alvo e a probabilidade de distribuição dessas cargas no alvo.

Tomando-se a média sobre um conjunto de campos de cor com paridade de carga par na equação (5.5) obtém-se a parte real da matriz S para o dipolo de cor, $D(\vec{r})$, que possui paridade par sob a transformação $r \rightarrow-r$. Por outro lado, neste estudo, flutuações com paridade de carga ímpar fornecem o valor esperado para a parte imaginária da matriz $\mathrm{S}, \operatorname{Im} S=O(\vec{r})$, que tem paridade ímpar sob a transformação $r \rightarrow-r$.

Claramente, a equação (5.5) pode ser convertida na seção de choque inclusiva para a produção de um hádron do tipo $h$ em uma colisão $p A,(p A \rightarrow h+X)$, considerando-se as convoluções com as distribuições de pártons no projétil, $f\left(x, Q^{2}\right)$, e um modelo para a função de fragmentação, $D_{h / f}\left(z, Q^{2}\right)$, responsável pela conversão do quark emergente 
$f$ em hádrons do tipo $h[73,452,453]$. Como resultado final, obtém-se exatamente o primeiro termo da equação (4.4).

Por se tratar de um estudo de cunho exploratório, aqui não se pretendeu comparar diretamente os resultados obtidos para $v_{n}\left(p_{T}\right)$ com os dados experimentais disponíveis e, portanto, as convoluções mencionadas acima foram desconsideradas. De fato, o objetivo deste estudo foi mais modesto: simplesmente nos empenhamos a tentar obter algum entendimento sobre comportamento dos harmônicos azimutais, $v_{n}$, a partir da equação (5.5).

Deve-se salientar que, embora a equação (5.5) inclua a contribuição de múltiplos espalhamentos do párton projétil com o núcleo alvo, assumimos, intrínsecamente, que o projétil é diluído e os efeitos não lineares no próton podem ser desprezados. É claro, esta pode não ser uma boa hipótese para colisões $p A$ com alta multiplicidade nas energias do LHC, exceto na região de fragmentação do próton [196].

Dessa forma, a distribuição de momento dos quarks espalhados pelos campos de cor do núcleo pode ser escrita como

$$
\begin{aligned}
\frac{d N}{k_{T} d k_{T} d \phi_{k}} & =\frac{1}{(2 \pi)^{2}} \int d^{2} r e^{-i \vec{k}_{T} \cdot \vec{r}} S(\vec{r}) \\
& =\frac{1}{(2 \pi)^{2}} \int d r r d \phi_{r} e^{-i k_{T} r \cos \left(\phi_{k}-\phi_{r}\right)} S\left(r, \phi_{r}\right)
\end{aligned}
$$

com $S(\vec{r})$ representando a matriz $\mathrm{S}$ de espalhamento de um dipolo de tamanho $r$ e orientação $\vec{r}, \vec{k}_{T}$ o momento do quark espalhado e $\phi_{k}$ o ângulo de espalhamento nesse processo.

Nessas condições, a distribuição de momento transversal das partículas produzidas é uma função real e portanto:

$$
S\left(r, \phi_{r}\right)=S^{*}\left(r, \phi_{r}+\pi\right)
$$

Uma vez que as partes real e imaginária de $S(\vec{r})$ na representação fundamental 
satisfazem as relações [450]

$$
\begin{aligned}
& S\left(r, \phi_{r}\right)=D\left(r, \phi_{r}\right)+i O\left(r, \phi_{r}\right) \\
& D\left(r, \phi_{r}\right)=D\left(r, \phi_{r}+\pi\right), \\
& O\left(r, \phi_{r}\right)=-O\left(r, \phi_{r}+\pi\right),
\end{aligned}
$$

a parte real, $D(\vec{r})$, é par sob a transformação $\phi_{r} \rightarrow \phi_{r}+\pi$ (ou $\vec{r} \rightarrow-\vec{r}$, equivalentemente) enquanto que a parte imaginária, $O(\vec{r})$, é ímpar.

Por outro lado, a matriz $S$ para um dipolo na representação adjunta (isto é, para um dipolo formado por dois gluons), $S_{a d j}$, é real. Quando escrita em termos das partes real e imaginária da matriz $S$ na representação fundamental (equação (5.10)), tem-se que:

$$
\begin{aligned}
\operatorname{Tr} L_{a d j} & =\left|\operatorname{Tr} L_{f}\right|^{2}-1, \quad L \in S U(N) \\
\frac{1}{N_{c}^{2}-1} \operatorname{Tr} L_{a d j} & =S_{a d j}=\frac{\left|\operatorname{Tr} L_{f}\right|^{2}-1}{N_{c}^{2}-1} \\
S_{a d j} & =\frac{N_{c}^{2}\left|\frac{1}{N_{c}} \operatorname{Tr} L_{f}\right|^{2}-1}{N_{c}^{2}-1}=\frac{N_{c}^{2}}{N_{c}^{2}-1}\left[\left|S_{f}\right|^{2}-1\right] \\
S_{\text {adj }}(\vec{r}) & =\frac{N_{c}^{2}\left[D^{2}(\vec{r})+O^{2}(\vec{r})\right]-1}{N_{c}^{2}-1} \rightarrow D^{2}(\vec{r})+O^{2}(\vec{r}),
\end{aligned}
$$

onde $S \equiv S_{f}$ e a expressão mais a direita aplica-se no regime de grandes $N_{c}$.

Claramente, $S_{\text {adj }}(\vec{r})=S_{\text {adj }}(-\vec{r})$ e, assim, apenas os harmônicos pares, $v_{2 n}$, são não nulos no caso de espalhamentos entre gluons do projétil e o núcleo alvo. Portanto, tem-se que neste caso os harmônicos azimutais ímpares devem ser gerados a partir de flutuações no núcleo alvo. Esta conclusão, de fato, não é nova. Na verdade já havia sido apontada em [169]. A geração de harmônicos azimutais ímpares através de algum outro mecanismo (que não as flutuações) é, atualmente, um problema que requer uma maior investigação teórica $[165,382]$. No estudo que aqui apresentamos, no entanto, consideramos apenas as assimetrias angulares geradas no estado inicial da colisão para espalhamentos entre quarks do projétil e o núcleo alvo. 


\subsection{Os harmônicos azimutais}

Pode-se definir diferentes momentos de assimetria, $v_{n}$, através da seguinte formulação:

$$
v_{n}\left(k_{T}\right)=\left\langle\cos n \phi_{k}\right\rangle=\frac{1}{\mathcal{N}} \int \frac{d \phi_{k}}{2 \pi} \cos \left(n \phi_{k}\right) \frac{d N}{k_{T} d k_{T} d \phi_{k}},
$$

onde

$$
\mathcal{N}=\int \frac{d \phi_{k}}{2 \pi} \frac{d N}{k_{T} d k_{T} d \phi_{k}}=\frac{1}{\pi} \frac{d N}{d k_{T}^{2}}
$$

Momentos pares (ímpares) possuem paridade positiva (negativa) uma vez que:

$$
\begin{aligned}
\left\langle\cos 2 n \phi_{k}\right\rangle & =+\left\langle\cos 2 n\left(\phi_{k}+\pi\right)\right\rangle, \\
\left\langle\cos (2 n+1) \phi_{k}\right\rangle & =-\left\langle\cos (2 n+1)\left(\phi_{k}+\pi\right)\right\rangle .
\end{aligned}
$$

No caso em que a amplitude de espalhamento, $S\left(r, \phi_{r}\right)$, é independente da orientação do dipolo, então, $v_{n}=0$ para todo $n$. Uma dependência angular na sua parte real, $D\left(r, \phi_{r}\right)$, gera momentos não nulos de paridade par, $v_{2 n}$, enquanto que uma dependência angular em $O\left(r, \phi_{r}\right)$ produz momentos ímpares $v_{2 n+1}$.

A seguir, consideramos modelos de dipolo para a matriz $S$, de modo a possibilitar a obtenção de resultados analíticos que permitam evidenciar as características qualitativas dos harmônicos azimutais, $v_{n}\left(k_{T}\right)$.

\subsection{Modelos para a parte real de $S\left(r, \phi_{r}\right)$}

\subsubsection{Modelo de dipolo quase-clássico}

O primeiro modelo de dipolo considerado nesta seção será o modelo McLerranVenugopalan (MV) [131-133], que foi construído assumindo-se um núcleo com grande número atômico composto inicialmente apenas por quarks de valência. Tais quarks 
de valência, que carregam grande fração de momento do alvo, são responsáveis pela emissão de gluons com pouca fração de momento (gluons "suaves" ou "soft"), gerando um meio de alta densidade gluônica. Uma vez que os gluons emitidos possuem pouca fração de momento, assume-se costumeiramente que esses quarks não sofrem alterações em suas trajetôrias ao emitirem ou absorverem um desses gluons; assim a aproximação eiconal na teoria do CGC torna-se aplicável.

No modelo MV, os gluons soft's são descritos por campos clássicos de cor $^{4}$ emitidos pelos quarks de valência que, por sua vez, são representados por uma densidade de carga de cor por unidade de área transversal e comprimento longitudinal $x^{-}$, $\rho^{a}\left(x^{-}, x_{\perp}\right)$. No limite de grandes densidades de cargas de cor, as flutuações dessas cargas são descritas por uma ação efetiva (do tipo gaussiana) segundo o teorema do limite central,

$$
S_{M V}[\rho]=\int d^{2} x_{\perp} \int_{-\infty}^{\infty} d x^{-} \frac{\rho^{a}\left(x^{-}, x_{\perp}\right) \rho^{a}\left(x^{-}, x_{\perp}\right)}{2 \mu^{2}\left(x^{-}\right)}
$$

onde $\mu^{2}\left(x^{-}\right) d x^{-}$é a densidade de fontes de carga cor por unidade de área transversal entre $x^{-}$e $x^{-}+d x^{-}$.

A equação (5.18) é frequentemente utilizada como "função peso" na equação (5.6) para calcular a média das configurações de cor do alvo no modelo MV. Para a função de dois pontos tem-se que

$$
\begin{aligned}
D(r) & \equiv \frac{1}{N_{c}}\left\langle\operatorname{Tr} V\left(x_{\perp}\right) V^{\dagger}\left(y_{\perp}\right)\right\rangle \\
& =\exp \left(-\frac{g^{2} C_{F}}{16 \pi} \int_{-\infty}^{\infty} x^{-} d x^{-} \mu^{2}\left(x^{-}\right) r^{2} \log \frac{1}{r \Lambda}\right) \\
& =\exp \left(-\frac{r^{2} Q_{s}^{2}}{4} \log \frac{1}{r \Lambda}\right),
\end{aligned}
$$

onde $r \equiv\left|x_{\perp}-y_{\perp}\right|, \Lambda$ é um corte no infravermelho introduzido para evitar o aparecimento de divergências e $Q_{s}^{2}$ é a escala de saturação dos quarks de valência.

\footnotetext{
${ }^{4}$ Isto é, o campo de cor do núcleo no regime de pequenos valores de $x$ é obtido resolvendo-se as equações clássicas de Yang-Mills.
} 
Os detalhes destes cálculos podem ser encontrados nas referências [35-43, 57, 58, 131-133,454]. É interessante notar que na expressão acima o modelo MV não possui nenhuma dependência energética, isto é, sua escala de saturação tem um valor fixo. Por esse motivo, este modelo é amplamente utilizado como condição inicial para a resolução das equações de evolução do CGC, as equações BK e JIMWLK, que se resolvidas podem possibilitar o comportamento quanto à dependência energética do modelo MV.

Na obtenção da expressão (5.19) calculou-se a média sobre todas as configurações de cor do alvo, conforme mencionado acima, e, portanto, $D(r)$ possui simetria rotacional. No limite de grandes momentos, $k_{T} \gg Q_{s}$, a distribuição de momento transversal decorrente de (5.19) é dada por [35-43,57, 58, 131-133,454]

$$
\frac{d N}{k_{T} d k_{T} d \phi_{k}}=\frac{1}{2 \pi} \frac{Q_{s}^{2}}{k_{T}^{4}}+\cdots
$$

No entanto, seguindo Kovner e Lublinsky [170], neste estudo considerou-se a média sobre todas as configurações de carga de cor no alvo para um ângulo relativo (entre o dipolo e o alvo) fixo. Desta maneira, a simetria rotacional local no plano transversal é espontaneamente quebrada em um evento particular pela direção de $\vec{E}$ em um dado domínio. De acordo com a referência [455], a presença de tal condensado pode fornecer uma possível explicação para os grandes valores de $v_{2}\{4\}$ observados em colisões de altas multiplicidades no LHC.

Considerando-se uma rotação global de cada evento, de forma que $\vec{E}=(E, 0)$ aponte na direção de $\vec{x}^{5}$, tem-se que $(\vec{r} \cdot \vec{E})^{2}=E^{2} r^{2} \cos ^{2} \phi_{r}$, com $\phi_{r}$ sendo o ângulo relativo entre o dipolo e o alvo. Assim, pode-se escrever [82]

$$
D(\vec{r})=\exp \left[-\frac{1}{4} r^{2} Q_{s}^{2}\left(1-\mathcal{A}+2 \mathcal{A} \cos ^{2} \phi_{r}\right) \log \frac{1}{\Lambda r}\right]
$$

onde $\mathcal{A}$ determina o grau de polarização dos campos de cor do alvo, com $\mathcal{A}=1$

\footnotetext{
${ }^{5}$ Este procedimento está de acordo com a convenção de que a distribuição de momento resultante é simétrica sob a transformação $\phi_{k} \rightarrow-\phi_{k}$ e que $v_{2} \equiv\left\langle\cos 2 \phi_{k}\right\rangle$ é maximizado.
} 
correspondendo a uma polarização perfeita, enquanto que com $\mathcal{A}=0$ essa expressão se reduz ao resultado não polarizado, dado pela equação (5.19).

Claramente $D(\vec{r})$ satisfaz (5.11), isto é, possui paridade par sob a transformação $\vec{r} \rightarrow-\vec{r}$ e, portanto, tem-se $v_{2 n+1}=0$ para todo $n$.

É importante enfatizar que em (5.21) não se efetuou explicitamente a média sobre múltiplos domínios de $\vec{E}$, como descrito em [170], mas sim assumiu-se a existência de um único domínio de $\vec{E}$ no núcleo alvo com polarização efetiva $\mathcal{A}$. Todavia, como será visto adiante, também considerou-se um (simples) modelo de domínio cujo o propósito é ilustrar as consequências da existência de diferentes domínios de $\vec{E}$ no núcleo alvo.

Considerando-se agora a equação (5.21), a distribuição de momento transversal dos quarks espalhados no regime $k_{T} \gg Q_{s}$ é dada por

$$
\frac{d N}{k_{T} d k_{T} d \phi_{k}}=\frac{1}{2 \pi} \frac{Q_{s}^{2}}{k_{T}^{4}}\left[1-2 \mathcal{A}\left(k_{T}\right)+4 \mathcal{A}\left(k_{T}\right) \cos ^{2} \phi_{k}\right]
$$

que, como esperado, possui uma dependência angular e conduz ao seguinte harmônico azimutal,

$$
v_{2}\left(k_{T}\right) \equiv\left\langle\cos 2 \phi_{k}\right\rangle=\mathcal{A}\left(k_{T}\right)
$$

com todos os outros $v_{n \neq 2}=0$. Assim, tem-se que $v_{2}\left(k_{T}\right)$ está relacionado com a polarização do campo do alvo na escala $k_{T}$.

Espera-se que $\mathcal{A}\left(k_{T}\right)$ decresça para valores de momento além da escala de saturação, já que os modos de pequenos comprimentos de onda de $\vec{E}$ devem possui orientações aleatórias, uma vez que não são afetados pelo comprimento de correlação.

Pode-se obter também a transformada de Fourier de (5.21) no regime $k_{T} \ll Q_{s}$. Neste caso o tamanho máximo da separação do dipolo, $r$, não é dado pela fase na transformada de Fourier, $r \sim 1 / k_{T}$, mas sim pela escala de saturação, $r \sim 1 / Q_{s}$. Nessas condições, obtém-se a seguinte distribuição de momento transversal

$$
\frac{d N}{k_{T} d k_{T} d \phi_{k}}=\frac{1}{\pi Q_{s}^{2} \log Q_{s} / \Lambda} \frac{1}{\sqrt{1-\mathcal{A}^{2}}} \exp \left[-\frac{k_{T}^{2}}{Q_{s}^{2} \log Q_{s} / \Lambda}\left(\frac{\cos ^{2} \phi_{k}}{1+\mathcal{A}}+\frac{\sin ^{2} \phi_{k}}{1-\mathcal{A}}\right)\right]
$$


O fator de normalização, $\mathcal{N}$, que figura na equação (5.15) é nesse caso

$$
\mathcal{N}=\frac{1}{\pi Q_{s}^{2} \log Q_{s} / \Lambda} \frac{1}{\sqrt{1-\mathcal{A}^{2}}} I_{0}\left(\frac{\mathcal{A}}{1-\mathcal{A}^{2}} \frac{k_{T}^{2}}{Q_{s}^{2} \log Q_{s} / \Lambda}\right) \exp \left[-\frac{k_{T}^{2}}{Q_{s}^{2} \log Q_{s} / \Lambda} \frac{1}{1-\mathcal{A}^{2}}\right]
$$

e, portanto, a distribuição angular normalizada é dada por

$$
\frac{1}{\mathcal{N}} \frac{d N}{k_{T} d k_{T} d \phi_{k}}=\frac{1}{I_{0}\left(\frac{\mathcal{A}}{1-\mathcal{A}^{2}} \frac{k_{T}^{2}}{Q_{s}^{2} \log Q_{s} / \Lambda}\right)} \exp \left[\frac{k_{T}^{2}}{Q_{s}^{2} \log Q_{s} / \Lambda} \frac{\mathcal{A}}{1-\mathcal{A}^{2}}\left(\cos ^{2} \phi_{k}-\sin ^{2} \phi_{k}\right)\right] .
$$

É importante notar que, neste caso, a amplitude de polarização é medida na escala $Q_{s}, \mathcal{A}=\mathcal{A}\left(Q_{s}\right)$ e é independente de $k_{T}$. Por fim, a equação (5.26) conduz aos seguintes harmônicos azimutais:

$$
\begin{aligned}
& v_{2}\left(k_{T}\right)=\frac{I_{1}\left(\frac{\mathcal{A}}{1-\mathcal{A}^{2}} \frac{k_{T}^{2}}{Q_{s}^{2} \log Q_{s} / \Lambda}\right)}{I_{0}\left(\frac{\mathcal{A}}{1-\mathcal{A}^{2}} \frac{k_{T}^{2}}{Q_{s}^{2} \log Q_{s} / \Lambda}\right)} \simeq \frac{\mathcal{A}}{2} \frac{k_{T}^{2}}{Q_{s}^{2} \log Q_{s} / \Lambda}, \\
& v_{4}\left(k_{T}\right)=\frac{I_{2}\left(\frac{\mathcal{A}}{1-\mathcal{A}^{2}} \frac{k_{T}^{2}}{Q_{s}^{2} \log Q_{s} / \Lambda}\right)}{I_{0}\left(\frac{\mathcal{A}}{1-\mathcal{A}^{2}} \frac{k_{T}^{2}}{Q_{s}^{2} \log Q_{s} / \Lambda}\right)} \simeq \frac{\mathcal{A}^{2}}{8}\left(\frac{k_{T}^{2}}{Q_{s}^{2} \log Q_{s} / \Lambda}\right)^{2},
\end{aligned}
$$

onde as expressões mais a direita são válidas quando a amplitude de polarização dos campos elétricos de cor do alvo é fraca, $\mathcal{A} \ll 1 \mathrm{e}$, portanto, tem-se que $v_{4}\left(k_{T}\right)$ não é nulo no regime de saturação, embora seja menor que $v_{2}\left(k_{T}\right)$ por uma potência adicional de $\mathcal{A}$ e de $k_{T}^{2} / Q_{s}^{2}$.

\subsubsection{Modelo de dipolo com dimensão anômala}

A amplitude de dipolo (5.21) adquire um expoente $\gamma$, conhecido como dimensão anômala, quando flutuações quânticas no regime de pequenas frações de momento, $x$, são consideradas,

$$
D(\vec{r})=\exp \left[-\frac{1}{4} r^{2} Q_{s}^{2}\left(1-\mathcal{A}+2 \mathcal{A} \cos ^{2} \phi_{r}\right) \log \frac{1}{\Lambda r}\right]^{\gamma}
$$


Na presença da dimensão anômala, logaritmos de $1 / r$ não afetam qualitativamente a distribuição de momento transversal, já que $\gamma<1$.

Nesta subseção, discutimos as distribuições angulares e seus momentos, $v_{n}\left(k_{T}\right)$, na presença da dimensão anômala. Restringimos-nos ao caso $\gamma=1 / 2$, pois este valor nos permite obter resultados analíticos. Os resultados provenientes desta análise devem ser suficientes para ilustrar o efeito qualitativo da dimensão anômala sobre $v_{n}\left(k_{T}\right)$.

No caso do limite isotrópico, onde $\mathcal{A}=0$, a distribuição de momento transversal tem a forma

$$
\frac{1}{\pi} \frac{d N}{d k_{T}^{2}}=\frac{1}{4 \pi} \frac{Q_{s}}{\left(k_{T}^{2}+Q_{s}^{2}\right)^{3 / 2}} .
$$

Na presença de um condensado polarizado, isto é para $\mathcal{A}>0$, é necessário considerar os limites $k_{T} \gg Q_{s}$ e $k_{T} \ll Q_{s}$ separadamente. No limite de grandes momentos pode-se expandir (5.29) em potências de $r$. A contribuição dominante $(\sim r)$ para $\mathcal{A} \ll 1$ fornece

$$
\frac{d N}{d^{2} k_{T}}=\left(1-\frac{3 \mathcal{A}}{2}+3 \mathcal{A} \cos ^{2} \phi_{k}\right) \frac{1}{4 \pi} \frac{Q_{s}}{k_{T}^{3}} \quad\left(k_{T} \gg Q_{s}, \mathcal{A} \ll 1\right) .
$$

A partir dessa expressão tem-se que

$$
\begin{aligned}
v_{2}\left(k_{T}\right) & =\frac{3}{4} \mathcal{A}\left(k_{T}\right) \\
v_{2 n \geq 4} & =0
\end{aligned}
$$

Portanto, para $k_{T} \gg Q_{s}$, a dimensão anômala não afeta o comportamento de $v_{2}\left(k_{T}\right)$ e $v_{4}\left(k_{T}\right)$ qualitativamente, como dito anteriormente.

Para $k_{T} \ll Q_{s}$ é possível calcular a integral radial da transformada de Fourier obtendo-se

$$
\frac{d N}{d^{2} k_{T}}=\frac{1}{\pi} \int_{-\pi}^{\pi} d \phi_{r} \frac{1}{\left(Q_{s} \sqrt{1+\mathcal{A} \cos 2 \phi_{r}}+2 i k_{T} \cos \left(\phi_{r}-\phi_{k}\right)\right)^{2}} .
$$

Expandindo-se o integrando em potências de $k_{T}$ encontra-se que a distribuição de 
momento transversal e o correspondente fator de normalização são dados por

$$
\begin{aligned}
\frac{d N}{d^{2} k_{T}} & =\frac{1}{Q_{s}^{2}}\left(\frac{2}{\sqrt{1-\mathcal{A}^{2}}}-12 \frac{k_{T}^{2}}{Q_{s}^{2}} \frac{1-\mathcal{A} \cos 2 \phi_{k}}{\left(1-\mathcal{A}^{2}\right)^{3 / 2}}\right) \\
\mathcal{N} & =\frac{1}{Q_{s}^{2}}\left(\frac{2}{\sqrt{1-\mathcal{A}^{2}}}-12 \frac{k_{T}^{2}}{Q_{s}^{2}} \frac{1}{\left(1-\mathcal{A}^{2}\right)^{3 / 2}}\right)
\end{aligned}
$$

Por fim, obtém-se os seguintes resultados para os harmônicos azimutais

$$
\begin{aligned}
v_{2}\left(k_{T}\right) & =\frac{6}{\mathcal{N}} \frac{k_{T}^{2}}{Q_{s}^{4}} \frac{\mathcal{A}}{\left(1-\mathcal{A}^{2}\right)^{3 / 2}}=\frac{3 \mathcal{A} k_{T}^{2}}{\left(1-\mathcal{A}^{2}\right) Q_{s}^{2}-6 k_{T}^{2}}, \\
v_{4}\left(k_{T}\right) & =0 .
\end{aligned}
$$

É importante notar que essa expressão é válida apenas enquanto $\mathcal{N}>0$, isto é para $k_{T}^{2} \ll\left(1-\mathcal{A}^{2}\right) Q_{s}^{2} / 6$. Portanto, para $\gamma=1 / 2$, tem-se que $v_{4}$ (e todos os harmônicos de mais alta ordem) são nulos na região de pequenos momentos transversais em contraste com o modelo clássico, onde $v_{4} \sim \mathcal{A}^{2} k_{T}^{4}$ (equação (5.28)).

A tabela 5.1 resume os resultados das equações $(5.23,5.27,5.28,5.32,5.33,5.37)$.

\begin{tabular}{c|c|c|c}
\hline & $v_{2}$ & $v_{4}$ & $\gamma$ \\
\hline$k_{T} \gg Q_{s}$ & $\mathcal{A}\left(k_{T}\right)$ & 0 & $\gamma \rightarrow 1$ \\
& $\mathcal{A}\left(k_{T}\right)$ & 0 & $\gamma=1 / 2$ \\
\hline$k_{T} \ll Q_{s}$ & $\mathcal{A} k_{T}^{2}$ & $\mathcal{A}^{2} k_{T}^{4}$ & $\gamma \rightarrow 1$ \\
& $\mathcal{A} k_{T}^{2}$ & 0 & $\gamma=1 / 2$ \\
\hline
\end{tabular}

Tabela 5.1: Síntese das contribuições dominantes em $k_{T}$ e $\mathcal{A}$ para $v_{2}$ and $v_{4}$.

A seguir, discutimos os modelos para a parte imaginária da amplitude de espalhamento de dipolo e os harmônicos azimutais resultantes.

\subsection{Modelos para a parte imaginária de $S\left(r, \phi_{r}\right)$}

Nesta seção consideramos a distribuição angular devido à parte imaginária da amplitude de espalhamento frontal de um dipolo de raio transversal $r, O(\vec{r})$. Restringindo- 
se à aproximação quase-clássica tem-se que [450]

$$
i O(\vec{r}) \sim i \vec{r} \cdot \vec{\nabla}_{b} D(\vec{r}, \vec{b})
$$

A constante de proporcionalidade nessa expressão foi calculada em [450] e será especificada posteriormente. Aqui, $\vec{b}$ denota a coordenada transversal do centro de massa do dipolo (relativo ao centro de núcleo): $\vec{b}=(\vec{x}+\vec{y}) / 2$, com $\vec{x}$ e $\vec{y}$ sendo as extremidades do dipolo. $D(\vec{r}, \vec{b})$, a parte real da matriz $\mathrm{S}$, depende de $\vec{b}$ através de $Q_{s}^{2}(\vec{b})$.

Daqui em diante considera-se a aproximação de que o núcleo é infinito e homogêneo de modo que, na média, $Q_{s}^{2}(\vec{b})=Q_{s}^{2}$ é constante. No entanto, o dipolo projétil ainda pode se acoplar com flutuações de pequenos comprimentos de onda no alvo e fornecer contribuições não nulas para $O(\vec{r})$. Para considerar o acoplamento das flutuações na escala do raio do dipolo, $r$, é necessário generalizar (5.39) para [82]

$$
i O(\vec{r}) \sim i \int_{\vec{y}}^{\vec{x}} d \vec{s} \cdot \vec{\nabla} D(\vec{r}, \vec{s})
$$

onde $\vec{s}$ é o vetor que conecta o quark e o antiquark que constituem o dipolo de cor.

No caso onde as flutuações ocorrem em escalas maiores que $r$ a equação (5.40) se reduz a (5.39), uma vez que o gradiente ao longo do dipolo pode ser calculado no ponto médio $\vec{b}$. Em um primeiro momento, assume-se que o campo elétrico possui polarização aleatória, portanto $\mathcal{A}=0$ na notação da seção anterior. Os efeitos do condensado induzido por $\vec{E}$ serão discutidos posteriormente. No limite de campos clássicos, $D(\vec{r}, \vec{s})$ é dado por $(5.21)$ e tem-se que

$$
i O(\vec{r}) \sim-\frac{i}{4} r^{2} \log \frac{1}{\Lambda r} e^{-\frac{1}{4} r^{2} Q_{s}^{2} \log \frac{1}{\Lambda r}} \int_{\vec{y}}^{\vec{x}} d \vec{s} \cdot \vec{\nabla} Q_{s}^{2}(\vec{s})
$$

Supondo-se que as flutuações no núcleo alvo são dominadas por um único modo, 
pode-se escrever

$$
\begin{aligned}
\frac{Q_{s}^{2}(\vec{s})}{Q_{s}^{2}} & =1+\int \frac{d^{2} q}{(2 \pi)^{2}} \delta f(\vec{q}) e^{i \vec{q} \cdot \vec{s}}, \\
\delta f(\vec{q}) & =\frac{(2 \pi)^{2}}{2} \mathcal{B}\left(q_{0}\right)\left[(1+i) \delta\left(\vec{q}-\vec{q}_{0}\right)+(1-i) \delta\left(\vec{q}+\vec{q}_{0}\right)\right]
\end{aligned}
$$

onde $\vec{q}_{0}=\left(q_{0}, 0\right)$ determina a escala onde acontecem as flutuações na escala de saturação e a direção de onde ocorre a quebra espontânea da simetria rotacional e $\mathcal{B}\left(q_{0}\right)$ representa a amplitude dessas flutuações (é importante notar que o ansatz proposto para as flutuações satisfaz a igualdade $\delta f(\vec{q})=\delta f^{*}(-\vec{q})$, de modo que $Q_{s}^{2}(\vec{s})$ é real).

A princípio, a direção do vetor de onda das flutuações, $\vec{q}$, pode apontar para qualquer direção e, portanto, não precisa necessariamente coincidir com a direção do condensado que dá origem aos harmônicos pares. Todavia, neste estudo, não foram consideradas possíveis correlações entre os "planos de evento" de $v_{n}$ e fixou-se $\vec{q}=\left(q_{0}, 0\right)$ de modo que $\vec{q}$ apontasse para a mesma direção induzida pelo condensado formado por $\vec{E}$ discutido na seção anterior.

Com este ansatz obtém-se,

$$
\begin{aligned}
\int_{\vec{y}}^{\vec{x}} d \vec{s} \cdot \vec{\nabla} Q_{s}^{2}(\vec{s}) & =-Q_{s}^{2} \mathcal{B}\left(q_{0}\right) \int_{\vec{y}}^{\vec{x}} d \vec{s} \cdot \vec{q}_{0}\left[\sin \left(\vec{q}_{0} \cdot \vec{s}\right)+\cos \left(\overrightarrow{q_{0}} \cdot \vec{s}\right)\right] \\
& =-2 Q_{s}^{2} \mathcal{B}\left(q_{0}\right) \sin \frac{\vec{q}_{0} \cdot \vec{r}}{2},
\end{aligned}
$$

onde parametrizou-se a linha de $\vec{y}$ para $\vec{x}$ como $\vec{s}(\sigma)=\vec{b}+\vec{r} \sigma$ com $\sigma \in\left[-\frac{1}{2}, \frac{1}{2}\right]$ e, então, fez-se $\vec{b}=0$.

Substituindo-se (5.45) em (5.41) tem-se

$$
i O(\vec{r}) \sim \frac{i}{2} r^{2} Q_{s}^{2} \mathcal{B}\left(q_{0}\right) \sin \left(\frac{1}{2} r q_{0} \cos \phi_{r}\right) \log \frac{1}{\Lambda r} e^{-\frac{1}{4} r^{2} Q_{s}^{2} \log \frac{1}{\Lambda r}}
$$

De modo a obter um entendimento qualitativo dos resultados apresentados, consideramos o limite de altos momentos ou, correspondentemente, pequenos dipolos, 
$r \ll 1 / Q_{s}$. Neste limite a exponencial em (5.46) pode ser ignorada resultando em

$$
i O(\vec{r}) \sim \frac{i}{2} r^{2} Q_{s}^{2} \mathcal{B}\left(q_{0}\right) \sin \left(\frac{1}{2} r q_{0} \cos \phi_{r}\right) \log \frac{1}{\Lambda r} \quad\left(r Q_{s} \ll 1\right)
$$

Uma flutuação com comprimento de onda muito grande (comparado com o tamanho do dipolo) corresponde a $r q_{0} \ll 1$. Utilizando-se a aproximação de pequenos ângulos, $\sin (\theta) \sim \theta$, é possível calcular a transformada de Fourier analiticamente obtendo-se

$$
\frac{d N}{d^{2} k_{T}} \sim \mathcal{B}\left(q_{0}\right) \frac{q_{0}}{k_{T}} \frac{Q_{s}^{2}}{k_{T}^{4}} \cos \left(\phi_{k}\right)
$$

De fato, o limite $r q_{0} \ll 1$ equivale ao cálculo do gradiente de $D(\vec{x}, \vec{y})$ no ponto médio $\vec{b}$, como na equação (5.39). Esta distribuição angular corresponde a $v_{1}\left(k_{T}\right) \neq 0$, evidentemente, porém fornecendo $v_{3}\left(k_{T}\right)=0$.

As flutuações que ocorrem na escala $r q_{0} \gtrsim 1$ podem ocasionar o aparecimento de harmônicos de mais alta ordem. Para ilustrar este fato, integra-se a equação (5.46) sob um espectro invariante de flutuações

$$
\mathcal{B}\left(q_{0}\right) \rightarrow \frac{1}{2} \mathcal{B} \int \frac{d q_{0}^{2}}{q_{0}^{2}}
$$

agindo na função seno.

É claro, ao realizar-se este procedimento, estamos nos restringindo ao caso extremo, onde todos os vetores de onda $\vec{q}_{0}$ estão apontando na mesma direção. Isto transforma a equação (5.46) em

$$
i O(\vec{r}) \sim \frac{i \pi}{4} r^{2} Q_{s}^{2} \mathcal{B} \operatorname{sgn}\left(\cos \phi_{r}\right) \log \frac{1}{\Lambda r} e^{-\frac{1}{4} r^{2} Q_{s}^{2} \log \frac{1}{\Lambda r}}
$$

É possível generalizar esta equação adicionando-se um corte do tipo exponencial para flutuações que ocorrem além de uma dada escala de momento $\left(Q_{c}\right)$

$$
\mathcal{B}\left(q_{0}\right) \rightarrow \frac{1}{2} \mathcal{B} \int \frac{d q_{0}^{2}}{q_{0}^{2}} e^{-q_{0} / Q_{c}}
$$


que, novamente, age na função seno na equação (5.46).

Assim, a parte imaginária da matriz $S$ com paridade de carga ímpar é dada por

$$
i O(\vec{r}) \sim \frac{i}{2} r^{2} Q_{s}^{2} \mathcal{B} \arctan \left(\frac{1}{2} r Q_{c} \cos \phi_{r}\right) \log \frac{1}{\Lambda r} e^{-\frac{1}{4} r^{2} Q_{s}^{2} \log \frac{1}{\Lambda r}}
$$

de modo que a equação (5.50) é reproduzida quando $Q_{c} \rightarrow \infty$.

Considerando-se o limite de pequenos dipolos, $r^{-1} \gg Q_{c}$ e $Q_{s}$, tem-se que

$$
i O(\vec{r}) \sim i r^{3} Q_{c} Q_{s}^{2} \mathcal{B} \cos \phi_{r}\left[1-\frac{r^{2}}{4}\left(\frac{Q_{c}^{2} \cos ^{2} \phi_{r}}{3}+Q_{s}^{2}\right)\right]
$$

A equação acima revela que $v_{1}$ é gerado em ordem $r^{3}$ enquanto que $v_{3}$ é gerado apenas em ordem $r^{5}$ e, portanto, decresce com uma potência adicional de $1 / k_{T}^{2}$ no limite de grandes momentos.

Finalmente, restaurando-se o fator multiplicativo de $i O(\vec{r})$ como dado na referência [450], tem-se:

$$
i O(\vec{r})=i \frac{\alpha_{s}\left(N_{c}^{2}-4\right)}{2^{6}} \mathcal{B} \arctan \left(\frac{1}{2} r Q_{c} \cos \phi_{r}\right) \rho^{2} e^{-\rho^{2}}
$$

com

$$
\rho^{2}=\frac{1}{4} r^{2} Q_{s}^{2} \log \frac{1}{\Lambda r}
$$

Nessa situação, o efeito do condensado gerado por $\vec{E}$ modifica a equação (5.55) para

$$
\rho^{2} \rightarrow \rho_{\mathcal{A}}^{2}=\rho^{2}\left(1-\mathcal{A}+2 \mathcal{A} \cos ^{2} \phi_{r}\right)
$$

Nessa equação assume-se que as flutuções que geram os harmônicos ímpares, $v_{2 n+1}$, estão perfeitamente alinhados na direção do condensado formado por $\vec{E}$, que, por sua vez, é responsáavel por gerar os momentos pares, $v_{2 n}$. Por simplicidade, assumiu-se aqui que este não é o caso e, ao invés disso, considerou-se que os planos de evento de $v_{2 n}$ e $v_{2 n+1}$ possuem orientações relativas aleatórias. Em outras palavras, os harmônicos pares e ímpares são produzidos de modo independente. Portanto, no que se segue, faremos uso da equação (5.55). 


\subsection{Resultados}

Nesta seção apresentamos os resultados numéricos para os harmônicos azimutais, $v_{n}$, dados por

$$
v_{n}(k)=e^{i \pi \delta_{n 1}}\left(2 \frac{d N}{d k^{2}}\right)^{-1} \int d \phi_{k} \frac{d N}{d^{2} k} \cos n \phi_{k} .
$$

Para efetuar tais cálculos discretizou-se o espaço das posições, $\vec{r}$, em uma rede bidimensional $\left(\vec{r}=\left(r_{x}, r_{y}\right)\right)$ e calculou-se a transformada de Fourier para a rede recíproca, isto é, uma rede bidimensional no espaço dos momentos, $\vec{k}$.

Mesmo quando $d N / d^{2} k$ é escolhido como sendo simétrico sob a transformação $\phi_{k} \rightarrow-\phi_{k}$, existe, em geral, uma fase indeterminada, $\exp (i \pi j)$, onde $j$ representa um número inteiro, que é escolhida por convenção. Como será visto adiante, o sinal negativo adicional na definição de $v_{1}$ é devido à convenção de que $v_{1}\left(p_{T}\right)<0$ na região de pequenos momentos, passando para $v_{1}\left(p_{T}\right)>0$ na região de grandes momentos.

No cálculo númerico de $d N / d^{2} k$ assumimos que $Q_{s} / \Lambda=10$, de modo que qualquer dependência com o corte no infravermelho, $\Lambda$, deve ser fraca. Também tomamos o cuidado de que a dimensão da rede por nós considerada, $L Q_{s} \sim 250$, excedesse em muito a escala física do problema. Por último, em todos os casos consideramos a escala de saturação da rede $Q_{s}^{L} \equiv a Q_{s} \ll 1$ (onde $a$ denota o espaçamento da rede no espaço das posições), de modo que os efeitos da discretização fossem pequenos.

De acordo com a equação (5.8), a distribuição de momento transversal, $d N / d^{2} k$, é obtida a partir da transformada de Fourier da matriz $S$ de um dipolo de cor, que consideramos aqui na aproximação de um modelo quase-clássico, dado por

$$
\operatorname{Re} S(\vec{r}) \equiv D(\vec{r})=e^{-\rho^{2}\left(1-\mathcal{A}+2 \mathcal{A} \cos ^{2} \phi_{r}\right)}
$$

e

$$
\operatorname{Im} S(\vec{r}) \equiv O(\vec{r})=\frac{\alpha_{s}\left(N_{c}^{2}-4\right)}{64} \mathcal{B} \arctan \left(\frac{1}{2} r Q_{c} \cos \phi_{r}\right) \rho^{2} e^{-\rho^{2}}
$$


com

$$
\rho^{2}=\frac{1}{4} r^{2} Q_{s}^{2} \log \left(e+\frac{1}{\Lambda r}\right)
$$

onde uma constante no argumento do logaritmo foi adicionada de modo a evitar uma troca não física de sinal no regime de baixíssimos momentos.

Nestes cálculos, foram considerados apenas alvos densos, para os quais $Q_{s} \gg \Lambda$, de forma que a região $r>1 / \Lambda$ não cumpra nenhum papel crucial.

Em princípio, o ângulo relativo entre o dipolo e a direção do condensado de campo elétrico $\vec{E}$, ( $\phi_{r}$, na equação (5.59)) pode ser deslocado por um ângulo aleatório, $\phi_{r} \rightarrow$ $\phi_{r}+\psi_{0}$, sem trazer nenhuma alteração na análise apresentada a seguir. Por fim, nos resultados a serem apresentados a seguir utilizou-se $\alpha_{s}=0.25$ e $N_{c}=3$, sendo que a amplitude de polarização do campo $\vec{E}$ e a intensidade das flutuações, $\mathcal{A}$ e $\mathcal{B}$, respectivamente, foram tratadas como parâmetros livres.

O principal objetivo da próxima seção é mostrar algumas características qualitativas das equações apresentadas acima. O presente estudo não tem a intenção de fornecer uma descrição quantitativa dos dados experimentais e se restringiu apenas aos harmônicos angulares de quarks espalhados elasticamente, omitindo assim convoluções da função de distribuição de pártons no próton projétil com funções de fragmentação párton $\rightarrow$ hádron. Todavia, de modo a possibilitar uma comparação, ainda que rudimentar, com os dados experimentais, reescalamos o momento transversal do hádron produzido através da relação $p_{T}=\langle z\rangle k_{T}=k_{T} / 2$.

\subsubsection{Harmônicos pares}

A Figura 5.3 mostra o resultado para $v_{2}\left(p_{T}\right)$ obtido a partir do modelo de dipolo quase-clássico. Nesta figura escalonou-se o momento medido experimentalmente, $p_{T}$, por $Q_{s}=1.3 \mathrm{GeV}$, de modo a se obter uma variável adimensional; aqui é importante notar que o valor escolhido para $Q_{s}$ é fortemente correlacionado com $\langle z\rangle=1 / 2$, assumido no rescalonamento do momento do quark de modo a simular (ainda que gros- 


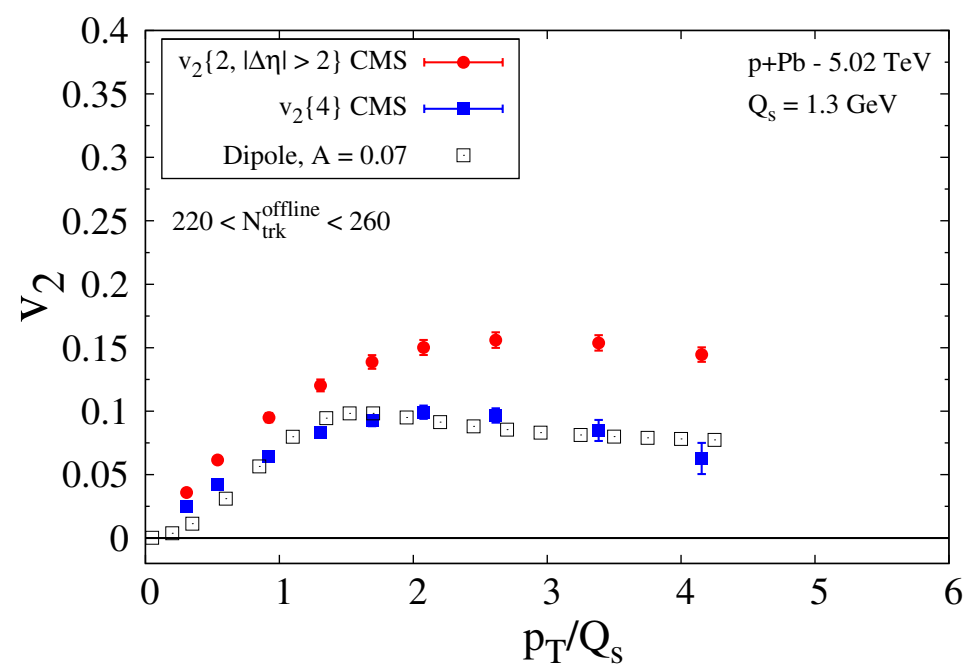

Figura 5.3: $v_{2}\left(p_{T}\right)$ obtido com o modelo de dipolo quase-clássico com amplitude de polarização do campo elétrico de cor $\mathcal{A}=7 \%$ (quadrados abertos). Os dados da colaboração CMS, $v_{2}\{2\}$ e $v_{2}\{4\}$, representados por cículos e quadrados cheios, respectivamente, correspondem a colisões $p P b$ com maior multiplicidade a $\sqrt{s}=$ $5.02 \mathrm{TeV}$.

seiramente) o efeito da hadronização. É claro, para se extrair um valor mais realístico de $Q_{s}$, seria necessário levar em consideração as convoluções de determinadas distribuições de pártons do próton e funções de fragmentação.

Para além da natureza qualitativa do resultado numérico apresentado, nota-se, a partir da equação (5.58), que o uso de uma amplitude de polarização do campo $\vec{E}$ de $\mathcal{A}=7 \%$, o modelo reproduz a magnitude e (ligeiramente) a dependência com $p_{T}$ dos dados experimentais. É oportuno mencionar que a dependência em $p_{T}$ dessa quantidade é obtida diretamente do modelo MV, ou seja, não foi obtida através do ajuste de outros parâmetros. Esta análise fornece uma primeira estimativa da magnitude de $\mathcal{A}$, que era, até então, desconhecida. Com esse mesmo valor para $\mathcal{A}$ obtivemos $v_{4}\left(p_{T}\right) \leq 1 \%$, como esperado a partir da discussão sobre os resultados analíticos apresentada nas seções anteriores. É importante mencionar que aqui não se efetuou uma média sobre múltiplos domínios de $\vec{E}$ explicitamente, isto é, considerou-se apenas a existência de um único domínio de $\vec{E}$ no núcleo alvo cuja polarização efetiva de $7 \%$ se ajusta aos dados experimentais de forma qualitativamente satisfatória. 
A seguir apresentamos um simples modelo de domínio cujo propósito é ilustrar os efeitos de se realizar uma média sobre diferentes domínios de $\vec{E}$ no núcleo alvo.

\subsubsection{Modelo de domínio}

Na subseção anterior considerou-se que o alvo continha um único domínio de $\vec{E}$, com polarização efetiva de $7 \%$. Este valor relativamente pequeno para $\mathcal{A}$ pode ser devido ao fato de se efetuar a média sobre diferentes domínios de $\vec{E}$ que apontam em diferentes direções. Este fato impediu a obtenção de predições para $v_{4}$. Aqui, apresentamos um simples modelo de domínio de modo a mostrar que a inclusão de diferentes domínios no núcleo alvo permite o fornecimento de predições para $v_{4}$.

Para a construção do modelo de domínio é importante introduzir os cumulantes de mais altas ordens, utilizados para descrever a correlação de $m$ partículas. Por exemplo, a correlação de duas partículas é definida como

$$
v_{n}^{2}\{2\} e^{i \psi} \equiv\left\langle e^{i n\left(\phi_{1}-\phi_{2}\right)}\right\rangle=\frac{1}{\mathcal{N}} \int \frac{d \phi_{1}}{2 \pi} \frac{d \phi_{2}}{2 \pi} e^{i n\left(\phi_{1}-\phi_{2}\right)} \frac{d N}{d \phi_{1} d^{2} k_{1}} \frac{d N}{d \phi_{2} d^{2} k_{2}}+\cdots
$$

com uma generalização direta para $m>2$ partículas correlacionadas.

A fase $\psi$ não é importante na expressão acima e, no lado direito desta equação, assumiu-se que a distribuição angular das duas partículas produzidas se fatoriza em um produto da distribuição angular de cada uma delas. As reticências indicam a existência de correções à hipótese da fatorização, que não estão sendo consideradas.

Se as duas partículas são produzidas a partir do mesmo domínio do alvo, obtémse $v_{n}\{2\}=v_{n}$, como na seção anterior. Por outro lado, caso pelo menos uma das partículas seja produzida em outro domínio não correlacionado, isto é com $\vec{E}$ apontando em uma direção aleatória, então $v_{n}\{2\}=0$, já que é necessário efetuar-se a média entre as diferentes direções dos planos de reação. Ao todo, para um núcleo contendo $N_{D}$ domínios, encontra-se que o harmônico $v_{n}\{m\}$ obtido a partir da correlação 
de $m$ partículas é dado por:

$$
v_{n}\{m\}=v_{n} N_{D}^{\frac{1-m}{m}}
$$

A Figura 5.4 mostra os resultados para $v_{2}\{4\}$ com um valor maior para a polarização dos campos elétricos de cor do alvo, $\mathcal{A}=0,15,0,20,0,25$ e com a magnitude global do resultados ajustada por $N_{D}$. Os parâmetros do modelo foram ajustados de modo a descrever os dados experimentais para $v_{2}\{4\}$ da melhor forma possível mas, ao mesmo tempo, sem superestimar os dados experimentais oriundos da correlação de duas partículas, $v_{2}\{2\}$ na região $p_{T} \lesssim Q_{s}{ }^{6}$.
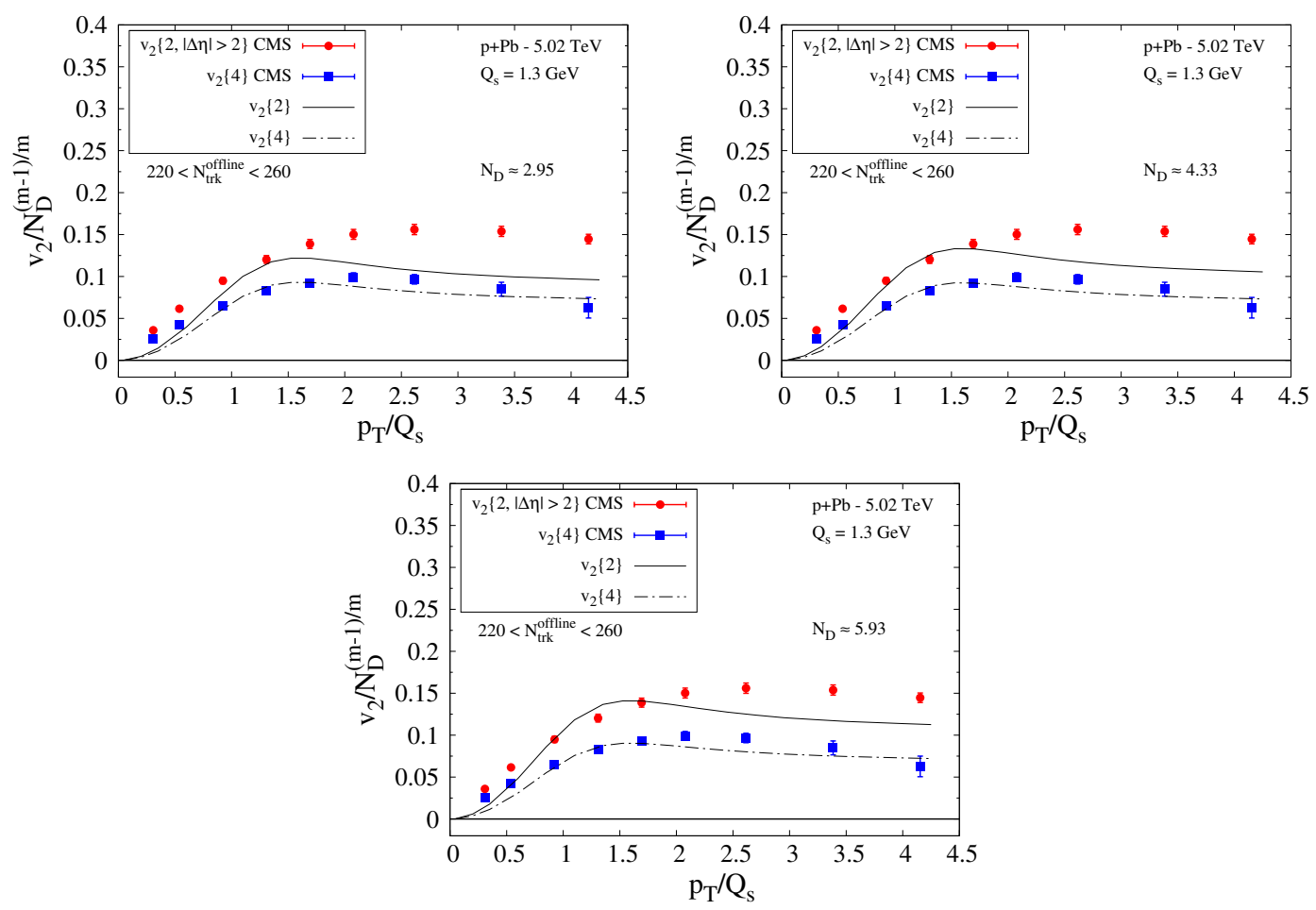

Figura 5.4: $v_{2}\left(p_{T}\right)$ a partir da correlação de duas e quatro partículas. O modelo de domínio com $N_{D}=2,95,4,33,5,93$ (e, correspondentemente, $\mathcal{A}=0,15,0,20$, $0,25)$ é comparado com os dados da colaboração CMS.

Nessa figura, a melhor descrição dos dados ocorre para $\mathcal{A} \simeq 0.20$ e $N_{D} \simeq 4.3$. Maiores valores de $\mathcal{A}$ resultam também em maiores valores de $N_{D}$ que, por sua vez,

\footnotetext{
${ }^{6}$ Não exigiu-se uma descrição perfeita de $v_{2}\{2\}$ em todo espectro de $p_{T}$ já que nesta análise não foram incluídas as correlações genuínas entre duas parículas, como indicado pelas reticências na equação (5.61).
} 
conduzem a uma separação muito grande entre $v_{2}\{2\}$ e $v_{2}\{4\}$, fazendo com que os resultados deixem de ser compatíveis com os dados experimentais. Neste modelo parece haver espaço para correlações genuínas, isto é, não fatorizáveis entre duas parículas no regime de altos valores de $p_{T}{ }^{7}$.

Uma vez determinados os valores de $\mathcal{A}$ e $N_{D}$, pode-se utilizar o mesmo modelo para o cálculo de $v_{4}\{2\}$ e $v_{4}\{4\}$. A colaboração ATLAS mediu experimentalmente $v_{4}\{2\}[183]$ e, portanto, é possível testar o modelo de domínio aqui utilizado. Como mostra a Figura 5.5, o modelo está de acordo com os dados experimentais de $v_{4}\{2\}$ na região de $Q_{s}$ considerada porém subestima os dados na região de altos momentos. Este fato pode ter origem na ausência de correlações não fatorizáveis, que não foram levadas em consideração.
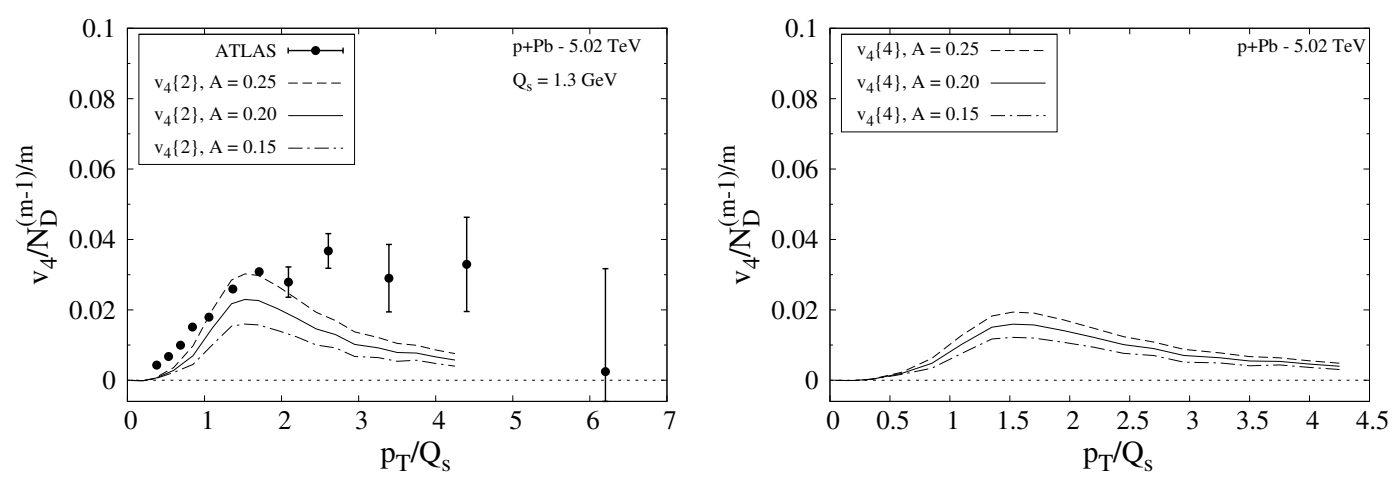

Figura 5.5: $v_{4}\left(p_{T}\right)$ a partir da correlação de duas e quatro partículas. No painel esquerdo o modelo de domínio com $N_{D}=2,95,4,33,5,93$ (e, correspondentemente, $\mathcal{A}=0,15,0,20,0,25$; de baixo para cima) é comparado com $v_{4}\{2\}$ medido pela colaboração ATLAS. No painel direito encontra-se a predição do modelo para $v_{4}\{4\}$ com as mesmas combinações de $\mathcal{A}$ e $N_{D}$.

A predição para $v_{4}\{4\}$ é apresentada na Figura 5.5 à direita. O modelo prevê $v_{4}\{4\} \lesssim 2 \%$ em todo alcance de $p_{T}$. Todavia, o fato mais importante é que, na região de $p_{T}>Q_{s}, v_{4}\{4\}$ claramente decresce com $p_{T}$. Esta é uma predição genérica da interação do dipolo com o campo elétrico, $\sim \vec{r} \cdot \vec{E}$.

A seguir, apresentamos os resultados obtidos para os dois primeiros harmônicos

\footnotetext{
${ }^{7}$ Tais correções foram calculadas posteriormente em [176] no limite de altos momentos e em [165] mostrou-se que, de fato, a sua inclusão permite uma descrição simultânea de $v_{2}\{2\}$ e $v_{2}\{4\}$ nesta mesma região.
} 
azimutais ímpares, $v_{1,3}\left(p_{T}\right)$.

\subsubsection{Harmônicos ímpares}

A dependência em $p_{T}$ de $v_{1}$ e $v_{3}$ para diferentes valores do corte $Q_{c}$ é apresentado na Figura 5.6 na qual a amplitude das flutuações de carga ímpar foi fixada em $\mathcal{B}=1$, já que o seu valor afeta somente a magnitude e não a dependência em $p_{T}$ das quantidades estudadas. Também mantivemos o valor $\mathcal{A}=7 \%$ da análise anterior e reescalamos o momento transversal quark espalhado para $p_{T}=k_{T} / 2$.
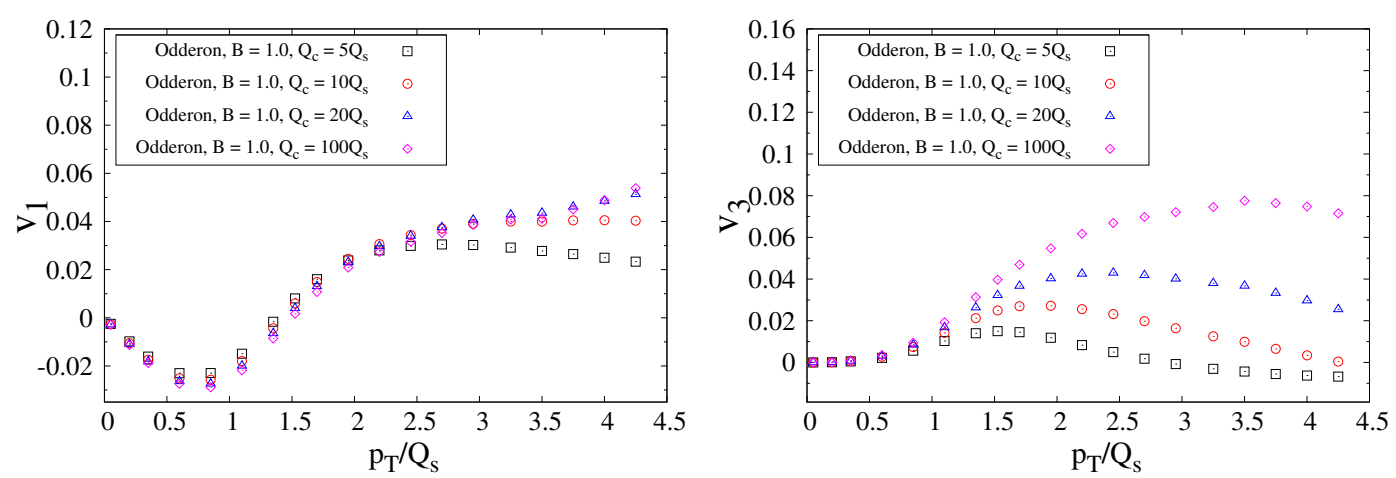

Figura 5.6: $v_{1}\left(p_{T}\right)$ e $v_{3}\left(p_{T}\right)$ obtido com o modelo quase-clássico para $O(\vec{r})$ com uma distribuição de flutuações de paridade de carga ímpar invariante de escala com um corte $Q_{c}$.

Nesta figura, observa-se que $v_{1}\left(p_{T}\right)$ depende fracamente do corte, $Q_{c}$, na região $p_{T} / Q_{s} \lesssim 2$. Já na região de grandes momentos, $v_{1}\left(p_{T}\right)$ cresce com $Q_{c}$ até o corte exceder, em muito, a escala de saturação. Por outro lado, na região de altos momentos, $v_{3}$ cresce rapidamente com $Q_{c}$. Este comportamento está de acordo com a discusão dos resultados analíticos apresentados na seção 5.5: flutuações com comprimento de onda da ordem do raio do dipolo geram $v_{1}$, mas não $v_{3}$.

Com base a essa análise é possível ajustar a amplitude das flutuações $(\mathcal{B})$ e o corte no espectro das flutuações $\left(Q_{c}\right)$ aos dados experimentais de $v_{1}\left(p_{T}\right)$ medidos pela colaboração ATLAS [183], considerando-se os eventos de altas multiplicidades em colisões $p P b$ a 5, $02 \mathrm{TeV}$ e, então, constatar o comportamento obtido para $v_{3}\left(p_{T}\right)$ com os dados 
experimentais da colaboração CMS [184]. Deve-se ter em mente, no entanto, que os dados experimentais mencionados correspondem aos harmônicos $v_{1,3}\{2\}$, isto é, são obtidos a partir da correlação de duas partículas, enquanto que o modelo aqui utilizado fornece harmônicos azimutais para a correlação de cada partícula produzida com a direção do plano de evento (dado por $\vec{q}_{0}$ neste caso) ou seja, $v_{1,3}\{1\}^{8}$.
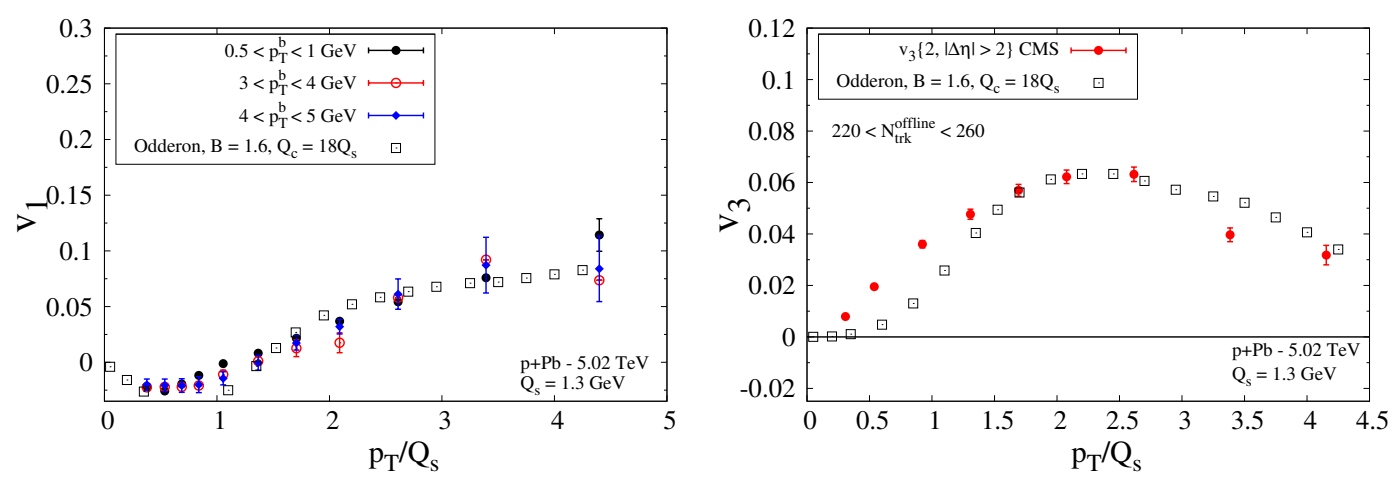

Figura 5.7: $v_{1}\left(p_{T}\right)$ e $v_{3}\left(p_{T}\right)$ obtidos com o modelo quase-clássico para $O(\vec{r})$ com uma amplitude de flutuação $\mathcal{B}=1.6$ e corte $Q_{c} / Q_{s}=18$. Os dados das colaborações ATLAS [183] $\left(v_{1}\right)$ e CMS [184] $\left(v_{3}\right)$ para $v_{1,3}\{2\}$ correspondem a eventos $p P b$ com a mais alta multiplicidade em $\sqrt{ } s=5.02 \mathrm{TeV}$.

A Figura 5.7 mostra que a magnitude de ambos, $v_{1}$ e $v_{3}$, é reproduzida pelo simples modelo utilizado para $O(\vec{r})$ com uma amplitude de flutuação $\mathcal{B}=1.6$. Além disso, um corte $Q_{c} \sim 18 Q_{s}$ conduz a uma descrição razoável de $v_{1}$ e $v_{3}$ na região de momentos transversais um pouco acima de $Q_{s}$. Claramente, nosso modelo não fornece nenhuma explicação da presença de tais flutuações e será importante entender isto detalhadamente no futuro.

Apresentamos, a seguir, as conclusões obtidas a partir dos estudos apresentados nessa tese bem como as perspectivas futuras.

\footnotetext{
${ }^{8} \mathrm{~A}$ geração de harmônicos azimutais ímpares a partir da correlação de $m$ partículas, $v_{2 n+1}\{m\}$, no estado inicial de colisões $p A$ ainda é uma questão em aberto.
} 


\section{Capítulo 6}

\section{Conclusões}

Nesta tese estudamos as interações hadrônicas a altas energias e pequenos valores da variável de Bjorken, $x$, considerando diversos observáveis físicos medidos nos aceleradores de partículas e também em eventos envolvendo raios cósmicos, onde as energias envolvidas são maiores que aquelas disponíveis nos aceleradores atuais.

No primeiro capítulo dessa tese, apresentamos uma revisão bibliográfica sobre a estrutura hadrônica, a QCD e suas equações de evolução, que predizem uma alta densidade partônica nos hádrons colidentes a altas energias. Já no segundo capítulo, apresentamos justamente os ingredientes utilizados na caracterização de sistemas hadrônicos com altas densidades partônicas. Mostramos que, nessas condições, as equações de evolução usuais da QCD para as distribuições de pártons nos hádrons colidentes tornam-se não lineares devido à inclusão do processo de recombinação de gluons, que conduz à saturação de pártons nos hádrons, tornando necessário o tratamento de múltiplos espalhamentos e efeitos de coerência. Apontamos que, em tais condições, espera-se a formação do CGC, um meio altamente denso onde os efeitos não lineares da QCD são de suma importância. Discutimos também os principais aspectos e ingredientes da abordagem do CGC, que é a teoria efetiva da QCD a altas energias e densidades partônicas, que se propõe a explicar as propriedades dinâmicas das interações hadrônicas no regime de saturação. 
No capítulo 3 , investigamos a influência dos efeitos da saturação de pártons nas seções de choque hadrônicas $p p, \bar{p} p$ e $p A$ e de fotoprodução $\gamma p$ e $\gamma \gamma$ com a energia da reação, $\sqrt{s}$. Para o cálculo dessas quantidades utilizamos um modelo de minijatos eiconalizado, onde as distribuições partônicas nos hádrons colidentes são os principais ingredientes. Os efeitos da saturação de pártons, nesse caso, foram considerados através da distribuição partônica EHKQS [70,71], cuja evolução na variável $Q^{2}$ obedece a equação GLRMQ, onde a primeira correção não linear às equações DGLAP, identificada como o processo de recombinação de gluons, está presente. Também calculamos tais quantidades utilizando as distribuições de pártons GRV98 [104] e CTEQ5L [91] que evoluem segundo as equações DGLAP (e, portanto, não consideram os efeitos da saturação) para efeitos de comparação. Para o cálculo da seção de choque em colisões $p A$ fizemos uso da aproximação de Glauber, que a relaciona com a seção de choque inelástica $p p$. Por fim, utilizamos o modelo aditivo de quarks e a dominância de mésons vetoriais para o cálculo das seções de choque de fotoprodução. Com essa abordagem além de respeitar o chamado limite de Froissart, conseguimos uma boa descrição dos dados experimentais das seções de choque totais hadrônicas e uma descrição razoável das seções de choque de fotoprodução quando os efeitos da saturação estão presentes. No entanto, as predições entre as diferentes dinâmicas da QCD (linear, sem efeito de saturação e não linear, com os efeitos da saturação de pártons ativos) se fez clara apenas na região de energia da física de raios cósmicos.

O capítulo 4 foi voltado para a produção de partículas nas regiões central e, principalmente, frontal de rapidez. Os resultados aqui apresentados foram obtidos no cenário do CGC [35-43,57,58,73-75], e o ferramental teórico utilizado nessa abordagem, onde os efeitos da saturação de pártons são considerados através dos chamados modelos de "dipolo de cor", foi detalhado na subseção 4.1.

Com uso do chamado "formalismo híbrido", na subseção 4.2 estudamos a distribuição de rapidez e de momento transversal da produção líquida de bárions (número de bárions menos o número de antibárions produzidos) em colisões $p p, p A$ e $A A$ e estimamos, pela primeira vez, o fator de modificação nuclear em colisões $p A$ para a 
produção de bárions dominantes. Como principal resultado desse estudo, mostramos que é possível descrever o espectro de momento transversal dessas partículas de modo mais eficiente, quando comparado a estudos já existentes na literatura [298,301]. Tal resultado foi obtido ao melhorarmos a descrição dos efeitos da dinâmica não linear presentes no hádron alvo fazendo uso do modelo de dipolo BUW. Ao variarmos a função de fragmentação (ingrediente responsável pela conversão dos pártons colidentes em hádrons observados experimentalmente), nossos resultados também permitiram inferir uma mudança no mecanismo responsável pela conversão párton-hádron com a energia do centro de massa da reação, indo do processo de coalescência (isto é, a recombinação de quarks dominantes que emergem próximos uns aos outros após o processo de espalhamento) para o processo de fragmentação independente. Estudamos também a chamada "anomalia bariônica" que ocorre quando a razão da seção de choque de produção de píons em relação à seção de choque de produção de bárions é próxima ou maior que a unidade e verificamos que essa razão decresce na região frontal, sugerindo que talvez a origem dessa "anomalia", observada nos dados experimentais, encontre-se na região central de rapidez. Por fim, observamos uma violação do scaling de Feynman no espectro em $x_{F}$ de partículas dominantes, compatível com outras abordagens $[315,316]$, onde diferentes mecanismos são responsáveis pela sua geração.

Ainda com o uso do formalismo híbrido da abordagem do CGC, na subseção 4.3 consideramos a produção de partículas pesadas na região frontal de rapidez, que também é influenciada por características específicas da dinâmica da QCD a altas energias. Em particular, estendemos a abordagem proposta em [348] para a produção de mésons $D$ a partir de quarks charme presentes no estado inicial da colisão, conhecidos como "charme intrínseco" (intrinsic cham - IC), calculando as distribuições de rapidez e em $x_{F}$ de tais mésons produzidos em colisões $p p$ nas energias do LHC e de raios cósmicos. A produção de mésons $D$ a partir de interações gluon-gluon, que é afetada pela existência de uma componente IC no próton projétil, também foi incluída nos cálculos mencionados. A componente IC é importante para o cálculo do fluxo de neutrinos produzidos através do decaimento de mésons pesados, os chamados "neutri- 
nos diretos" [329]. Como principais resultados desse estudo, tem-se que a distribuição em $x_{F}$ dos mésons $D$ é aumentada pela presença da componente IC e que esse aumento ocorre na região cinemática onde tais mésons fornecem a contribuição dominante para o fluxo de neutrinos diretos. Consequentemente, a inclusão de uma componente IC nos cálculos dessa quantidade pode ser importante para estimar o fluxo de neutrinos diretos produzidos na atmosfera terrestre, observável esse que pode ser medido pela colaboração IceCube.

Em seguida, apresentamos um estudo sobre o comportamento do momento transversal médio de partículas carregadas produzidas em colisões $p p$ e $p A$ com a rapidez e com a energia da colisão, $\left\langle p_{T}(y, \sqrt{s})\right\rangle[80]$. Assim como nos estudos anteriores, também fizemos uso do formalismo híbrido no cálculo dessa quantidade. Os resultados que obtivemos demonstraram que a razão $R=\left\langle p_{T}(y, \sqrt{s})\right\rangle /\left\langle p_{T}(0, \sqrt{s})\right\rangle$ cresce com a energia para uma rapidez fixa, porém decresce com a rapidez para uma energia fixa e, portanto, tem um comportamento similar aquele obtido via modelos hidrodinâmicos, ao contrário do que apontavam os resultados qualitativos apresentados na literatura [375]. Nossa análise indicou que esse decréscimo vem do tratamento da dinâmica da QCD a altas energias, da inclusão do processo de fragmentação e de restrições cinemáticas associadas aos limites do espaço de fase, que são alcançados a altissímos valores de rapidez.

Encerrando o capítulo 4, na subseção 4.5 consideramos a produção de partículas em colisões $p p, p A$ e $A A$ na região central de rapidez. Nesse caso, o formalismo híbrido utilizado nos estudos anteriores deixa de ser válido e fizemos uso do chamado formalismo da "fatorização $k_{T}$ " da abordagem do CGC para descrever a dinâmica da produção de partículas. Em particular, a seção de choque invariante da produção de gluons nesse formalismo foi derivada em [74] considerando-se o acoplamento forte, $\alpha_{s}$, constante (equação (4.2)). Os efeitos da inclusão das correções em $\alpha_{s}$ nesse formalismo foram tratados recentemente em [76], onde uma expressão corrigida para tal seção de choque, a priori válida em todo o espaço de fase, foi proposta (equação (4.22)). Nessa seção apresentamos, pela primeira vez, uma análise qualitativa das implicações dessas 
correções em observáveis físicos medidos em aceleradores de partículas.

Considerando suposições simples para a geometria nuclear em colisões $p A$ e $A A$ e para as distribuições de gluons não integradas (que figuram nas equações (4.2) e (4.22)), estimamos a seção de choque para a produção de hádrons nas colisões acima mencionadas e os resultados foram comparados com aqueles obtidos a partir do uso da fórmula da fatorização $k_{T}$ sem correções em $\alpha_{s}$ em duas situações distintas, com o acoplamento fixo e com duas diferentes prescrições para a escolha das escalas de momento em $\alpha_{s}\left(Q^{2}\right)$. Mostramos que o impacto dessas correções nas distribuições de pseudorapidez e multiplicidade de hádrons carregados é pequeno e que as predições das diferentes abordagens para tais quantidades são similares. Constatamos também que a fórmula corrigida produz uma descrição satisfatória dos dados experimentais, encontrando-se a principal diferença entre essas abordagens na dependência dos observáveis com a energia, onde a fórmula corrigida prediz uma dependência mais fraca.

No quinto capítulo dessa tese, apresentamos um estudo onde exploramos algumas características qualitativas de ideias em desenvolvimento: a geração de assimetrias azimutais (diretamente no espaço dos momentos) no estado inicial de colisões $p A$ na abordagem de dipolos de cor. Seguindo a referência [170], fizemos uso de uma amplitude de dipolo quase-clássica que conduz à quebra espontânea da simetria rotacional em um espaço bidimensional devido à formação de um condensado do campo elétrico de cor, $\vec{E}$. Como principal resultado desse estudo, nossas estimativas numéricas indicam que o modelo é apto a descrever a dependência da assimetria elíptica com $p_{T}$, $v_{2}\left(p_{T}\right)$, obtida a partir da correlação de quatro partículas.

Também abordamos os momentos azimutais de paridade ímpar, $v_{2 n+1}$, que, aqui, surgem a partir de flutuações na escala de saturação dependente do partâmetro de impacto na componente imaginária da matriz $S$. Fixando-se dois parâmetros, a amplitude dessas flutuações, $\mathcal{B}$, e a escala de momento em que essas flutuações deixam de existir, $Q_{c}$, mostramos que o simples modelo por nós utilizado fornece uma descrição simultânea e razoável de $v_{1}\{2\}\left(p_{T}\right)$ e $v_{3}\{2\}\left(p_{T}\right)$ resultantes de eventos de altas multiplicidades em colisões $p P b$ medidos no LHC. Enfatizamos, no entanto, que seria 
mais apropriado comparar esses nossos resultados com $v_{1}\left(p_{T}\right)$ e $v_{3}\left(p_{T}\right)$ obtidos a partir da correlação de quatro ou mais partículas, medidas que, infelizmente, ainda não se encontram disponíveis.

Existem, é claro, extensões para alguns dos trabalhos aqui apresentados. Uma continuação natural do estudo apresentado na subseção 4.3, por exemplo, é efetuar o cálculo do fluxo de neutrinos diretos incluindo-se uma componente IC e estudar como esse observável é modificado pela inclusão de tal componente. Já o estudo da subseção 4.5, que foi de cunho qualitativo, forneceu importantes resultados que motivam um cálculo mais robusto, considerando-se distribuições não integradas de gluons mais realísticas e um tratamento igualmente mais preciso da geometria nuclear. Em ambos os casos temos intenção de investigá-las e estudá-las com maiores detalhes no futuro.

Por último, conforme já apontado, o estudo apresentado no capítulo 5 é de cunho qualitativo e exploratório, e ainda existem diversos outros estudos a serem feitos antes que uma comparação quantitativa com os dados experimentais possa ser realizada. Por exemplo, nessa tese não abordamos de maneira alguma a natureza dos eventos de altas multiplicidades em colisões $p P b$ que apresentam (grande) assimetria azimutal. Esse procedimento requereria melhores modelos para as partes real e imaginária da amplitude de espalhamento dipolo-hádron, que está além da dimensão das investigações que aqui nos propusemos fazer, sob conta que seria necessário obter algum entendimento teórico mais refinado para a amplitude e para o corte das flutuações que geram os harmônicos ímpares, que, aqui, foram obtidos de maneira fenomenológica.

Com certos cuidados, atenção e empenho, pretendemos voltar nossas atenções para os estudos acima mencionados no futuro. 


\section{Bibliografia}

[1] S. L. Glashow, "Partial Symmetries of Weak Interactions," Nucl. Phys., vol. 22, pp. 579-588, 1961.

[2] S. Weinberg, "A Model of Leptons," Phys. Rev. Lett., vol. 19, pp. 1264-1266, 1967.

[3] M. Gell-Mann, "A Schematic Model of Baryons and Mesons," Phys. Lett., vol. 8, pp. 214-215, 1964.

[4] S. Weinberg, "Nonabelian Gauge Theories of the Strong Interactions," Phys. Rev. Lett., vol. 31, pp. 494-497, 1973.

[5] F. Englert and R. Brout, "Broken Symmetry and the Mass of Gauge Vector Mesons," Phys. Rev. Lett., vol. 13, pp. 321-323, 1964.

[6] P. W. Higgs, "Broken symmetries, massless particles and gauge fields," Phys. Lett., vol. 12, pp. 132-133, 1964.

[7] P. W. Higgs, "Broken Symmetries and the Masses of Gauge Bosons," Phys. Rev. Lett., vol. 13, pp. 508-509, 1964.

[8] G. S. Guralnik, C. R. Hagen, and T. W. B. Kibble, "Global Conservation Laws and Massless Particles," Phys. Rev. Lett., vol. 13, pp. 585-587, 1964.

[9] P. W. Higgs, "Spontaneous Symmetry Breakdown without Massless Bosons," Phys. Rev., vol. 145, pp. 1156-1163, 1966. 
[10] T. W. B. Kibble, "Symmetry breaking in nonAbelian gauge theories," Phys. Rev., vol. 155, pp. 1554-1561, 1967.

[11] D. J. Gross and F. Wilczek, "Ultraviolet Behavior of Nonabelian Gauge Theories," Phys. Rev. Lett., vol. 30, pp. 1343-1346, 1973.

[12] D. J. Gross and F. Wilczek, "Asymptotically Free Gauge Theories. 1," Phys. Rev., vol. D8, pp. 3633-3652, 1973.

[13] H. D. Politzer, "Reliable Perturbative Results for Strong Interactions?," Phys. Rev. Lett., vol. 30, pp. 1346-1349, 1973.

[14] K. G. Wilson, "Confinement of Quarks," Phys. Rev., vol. D10, pp. 2445-2459, 1974. [,45(1974)].

[15] V. N. Gribov and L. N. Lipatov, "Deep inelastic e p scattering in perturbation theory," Sov. J. Nucl. Phys., vol. 15, pp. 438-450, 1972. [Yad. Fiz.15,781(1972)].

[16] L. N. Lipatov, "The parton model and perturbation theory," Sov. J. Nucl. Phys., vol. 20, pp. 94-102, 1975. [Yad. Fiz.20,181(1974)].

[17] G. Altarelli and G. Parisi, "Asymptotic Freedom in Parton Language," Nucl. Phys., vol. B126, pp. 298-318, 1977.

[18] Y. L. Dokshitzer, "Calculation of the Structure Functions for Deep Inelastic Scattering and e+ e- Annihilation by Perturbation Theory in Quantum Chromodynamics.," Sov. Phys. JETP, vol. 46, pp. 641-653, 1977. [Zh. Eksp. Teor. Fiz.73,1216(1977)].

[19] E. A. Kuraev, L. N. Lipatov, and V. S. Fadin, "Multi - Reggeon Processes in the Yang-Mills Theory," Sov. Phys. JETP, vol. 44, pp. 443-450, 1976. [Zh. Eksp. Teor. Fiz.71,840(1976)]. 
[20] E. A. Kuraev, L. N. Lipatov, and V. S. Fadin, "The Pomeranchuk Singularity in Nonabelian Gauge Theories," Sov. Phys. JETP, vol. 45, pp. 199-204, 1977. [Zh. Eksp. Teor. Fiz.72,377(1977)].

[21] I. I. Balitsky and L. N. Lipatov, "The Pomeranchuk Singularity in Quantum Chromodynamics," Sov. J. Nucl. Phys., vol. 28, pp. 822-829, 1978. [Yad. Fiz.28,1597(1978)].

[22] I. Abt et al., "Measurement of the proton structure function F2 (x, Q**2) in the low x region at HERA," Nucl. Phys., vol. B407, pp. 515-538, 1993.

[23] S. Aid et al., "A Measurement and QCD analysis of the proton structure function f2 (x, q**2) at HERA," Nucl. Phys., vol. B470, pp. 3-40, 1996.

[24] C. Adloff et al., "A Measurement of the proton structure function f2 (x, q**2) at low $\mathrm{x}$ and low q**2 at HERA," Nucl. Phys., vol. B497, pp. 3-30, 1997.

[25] C. Adloff et al., "Measurement of neutral and charged current cross-sections in positron proton collisions at large momentum transfer," Eur. Phys. J., vol. C13, pp. 609-639, 2000.

[26] C. Adloff et al., "Measurement of neutral and charged current cross-sections in electron - proton collisions at high $Q^{2}, "$ Eur. Phys. J., vol. C19, pp. 269-288, 2001.

[27] C. Adloff et al., "Deep inelastic inclusive e p scattering at low x and a determination of alpha(s)," Eur. Phys. J., vol. C21, pp. 33-61, 2001.

[28] C. Adloff et al., "On the rise of the proton structure function $\mathrm{F}(2)$ towards low x," Phys. Lett., vol. B520, pp. 183-190, 2001.

[29] M. Derrick et al., "Measurement of the proton structure function F2 in e p scattering at HERA," Phys. Lett., vol. B316, pp. 412-426, 1993. 
[30] M. Derrick et al., "Measurement of the F2 structure function in deep inelastic e+ p scattering using 1994 data from the ZEUS detector at HERA," Z. Phys., vol. C72, pp. 399-424, 1996.

[31] J. Breitweg et al., "ZEUS results on the measurement and phenomenology of F(2) at low $\mathrm{x}$ and low Q**2," Eur. Phys. J., vol. C7, pp. 609-630, 1999.

[32] S. Chekanov et al., "Measurement of the neutral current cross-section and F(2) structure function for deep inelastic e + p scattering at HERA," Eur. Phys. J., vol. C21, pp. 443-471, 2001.

[33] L. V. Gribov, E. M. Levin, and M. G. Ryskin, "Semihard Processes in QCD," Phys. Rept., vol. 100, pp. 1-150, 1983.

[34] A. H. Mueller and J.-w. Qiu, "Gluon Recombination and Shadowing at Small Values of x," Nucl. Phys., vol. B268, pp. 427-452, 1986.

[35] A. Krasnitz and R. Venugopalan, "The Initial energy density of gluons produced in very high-energy nuclear collisions," Phys. Rev. Lett., vol. 84, pp. 4309-4312, 2000 .

[36] E. Levin and K. Tuchin, "Solution to the evolution equation for high parton density QCD," Nucl. Phys., vol. B573, pp. 833-852, 2000.

[37] E. Iancu and L. D. McLerran, "Saturation and universality in QCD at small x," Phys. Lett., vol. B510, pp. 145-154, 2001.

[38] D. Kharzeev and M. Nardi, "Hadron production in nuclear collisions at RHIC and high density QCD," Phys. Lett., vol. B507, pp. 121-128, 2001.

[39] D. Kharzeev and E. Levin, "Manifestations of high density QCD in the first RHIC data," Phys. Lett., vol. B523, pp. 79-87, 2001. 
[40] D. Kharzeev, E. Levin, and M. Nardi, "QCD saturation and deuteron nucleus collisions," Nucl. Phys., vol. A730, pp. 448-459, 2004. [Erratum: Nucl. Phys.A743,329(2004)].

[41] F. Gelis, E. Iancu, J. Jalilian-Marian, and R. Venugopalan, "The Color Glass Condensate," Ann. Rev. Nucl. Part. Sci., vol. 60, pp. 463-489, 2010.

[42] E. Iancu, A. Leonidov, and L. McLerran, "The Color glass condensate: An Introduction," in QCD perspectives on hot and dense matter. Proceedings, NATO Advanced Study Institute, Summer School, Cargese, France, August 6-18, 2001, pp. $73-145,2002$.

[43] E. Iancu and R. Venugopalan, "The Color glass condensate and high-energy scattering in QCD," in In *Hwa, R.C. (ed.) et al.: Quark gluon plasma* 2493363, 2003.

[44] I. Balitsky, "Operator expansion for high-energy scattering," Nucl. Phys., vol. B463, pp. 99-160, 1996.

[45] I. Balitsky, "Factorization for high-energy scattering," Phys. Rev. Lett., vol. 81, pp. 2024-2027, 1998.

[46] I. Balitsky, "Effective field theory for the small $\mathrm{x}$ evolution," Phys. Lett., vol. B518, pp. 235-242, 2001.

[47] Y. V. Kovchegov, "Small x F(2) structure function of a nucleus including multiple pomeron exchanges," Phys. Rev., vol. D60, p. 034008, 1999.

[48] J. Jalilian-Marian, A. Kovner, L. D. McLerran, and H. Weigert, "The Intrinsic glue distribution at very small x," Phys. Rev., vol. D55, pp. 5414-5428, 1997.

[49] J. Jalilian-Marian, A. Kovner, A. Leonidov, and H. Weigert, "The BFKL equation from the Wilson renormalization group," Nucl. Phys., vol. B504, pp. 415431, 1997. 
[50] J. Jalilian-Marian, A. Kovner, A. Leonidov, and H. Weigert, "The Wilson renormalization group for low x physics: Towards the high density regime," Phys. Rev., vol. D59, p. 014014, 1998.

[51] J. Jalilian-Marian, A. Kovner, A. Leonidov, and H. Weigert, "Unitarization of gluon distribution in the doubly logarithmic regime at high density," Phys. Rev., vol. D59, p. 034007, 1999. [Erratum: Phys. Rev.D59,099903(1999)].

[52] J. Jalilian-Marian, A. Kovner, and H. Weigert, "The Wilson renormalization group for low x physics: Gluon evolution at finite parton density," Phys. Rev., vol. D59, p. 014015, 1998.

[53] E. Iancu, A. Leonidov, and L. D. McLerran, "Nonlinear gluon evolution in the color glass condensate. 1.," Nucl. Phys., vol. A692, pp. 583-645, 2001.

[54] E. Ferreiro, E. Iancu, A. Leonidov, and L. McLerran, "Nonlinear gluon evolution in the color glass condensate. 2.," Nucl. Phys., vol. A703, pp. 489-538, 2002.

[55] E. Iancu, A. Leonidov, and L. D. McLerran, "The Renormalization group equation for the color glass condensate," Phys. Lett., vol. B510, pp. 133-144, 2001.

[56] A. H. Mueller, "A Simple derivation of the JIMWLK equation," Phys. Lett., vol. B523, pp. 243-248, 2001.

[57] F. Gelis, T. Lappi, and R. Venugopalan, "High energy scattering in Quantum Chromodynamics," Int. J. Mod. Phys., vol. E16, pp. 2595-2637, 2007.

[58] J. Jalilian-Marian and Y. V. Kovchegov, "Saturation physics and deuteron-Gold collisions at RHIC," Prog. Part. Nucl. Phys., vol. 56, pp. 104-231, 2006.

[59] L. McLerran, "What is the evidence for the color glass condensate?," in Structure and dynamics of elementary matter. Proceedings, NATO Advanced Study Institute, Camyuva-Kemer, Turkey, September 22-October 2, 2003, pp. 183-205, 2004. 
[60] R. C. Hwa, C. B. Yang, and R. J. Fries, "Forward production in d+Au collisions by parton recombination," Phys. Rev., vol. C71, p. 024902, 2005.

[61] B. Z. Kopeliovich, J. Nemchik, I. K. Potashnikova, M. B. Johnson, and I. Schmidt, "Breakdown of QCD factorization at large Feynman x," Phys. Rev., vol. C72, p. 054606, 2005.

[62] J.-w. Qiu and I. Vitev, "Coherent QCD multiple scattering in proton-nucleus collisions," Phys. Lett., vol. B632, pp. 507-511, 2006.

[63] F. Caola and S. Forte, "Geometric Scaling from GLAP evolution," Phys. Rev. Lett., vol. 101, p. 022001, 2008.

[64] N. N. Nikolaev and B. G. Zakharov, "Color transparency and scaling properties of nuclear shadowing in deep inelastic scattering," Z. Phys., vol. C49, pp. 607618, 1991.

[65] N. Nikolaev and B. G. Zakharov, "Pomeron structure function and diffraction dissociation of virtual photons in perturbative QCD," Z. Phys., vol. C53, pp. 331-346, 1992.

[66] N. N. Nikolaev and B. G. Zakharov, "The Triple pomeron regime and the structure function of the pomeron in the diffractive deep inelastic scattering at very small x," Z. Phys., vol. C64, pp. 631-652, 1994.

[67] A. H. Mueller, "Soft gluons in the infinite momentum wave function and the BFKL pomeron," Nucl. Phys., vol. B415, pp. 373-385, 1994.

[68] A. H. Mueller, "Unitarity and the BFKL pomeron," Nucl. Phys., vol. B437, pp. 107-126, 1995.

[69] A. H. Mueller and B. Patel, "Single and double BFKL pomeron exchange and a dipole picture of high-energy hard processes," Nucl. Phys., vol. B425, pp. 471488, 1994. 
[70] K. J. Eskola, H. Honkanen, V. J. Kolhinen, J.-w. Qiu, and C. A. Salgado, "Nonlinear corrections to the DGLAP equations in view of the hera data," Nucl. Phys., vol. B660, pp. 211-224, 2003.

[71] "Urhic group / nonlinear parton distributions." https://www.jyu.fi/ fysiikka/en/research/highenergy/urhic/EHKQS/. Accessed: 2017-04-10.

[72] A. V. Giannini, "O efeito da saturação de gluons no comportamento das seções de choque próton-próton, próton-núcleo e próton-ar," Master's thesis, Universidade de São Paulo, 2012.

[73] A. Dumitru, A. Hayashigaki, and J. Jalilian-Marian, "The Color glass condensate and hadron production in the forward region," Nucl. Phys., vol. A765, pp. 464$482,2006$.

[74] Y. V. Kovchegov and K. Tuchin, "Inclusive gluon production in DIS at high parton density," Phys. Rev., vol. D65, p. 074026, 2002.

[75] M. A. Braun, "Inclusive jet production on the nucleus in the perturbative QCD with $N_{c} \rightarrow$ infty," Phys. Lett., vol. B483, pp. 105-114, 2000.

[76] W. A. Horowitz and Y. V. Kovchegov, "Running Coupling Corrections to High Energy Inclusive Gluon Production," Nucl. Phys., vol. A849, pp. 72-97, 2011.

[77] A. V. Giannini and F. O. Duraes, "Nonlinear effects and the behavior of total hadronic and photonic cross sections," Phys. Rev., vol. D88, p. 114004, 2013.

[78] F. O. Duraes, A. V. Giannini, V. P. Goncalves, and F. S. Navarra, "Baryon stopping in the Color Glass Condensate formalism: A phenomenological study," Phys. Rev., vol. C89, no. 3, p. 035205, 2014.

[79] F. Carvalho, A. V. Giannini, V. P. Goncalves, and F. S. Navarra, "D - meson production at very forward rapidities: estimating the intrinsic charm contribution," arXiv:1701.08451, 2017. 
[80] F. O. Durães, A. V. Giannini, V. P. Goncalves, and F. S. Navarra, "Rapidity dependence of the average transverse momentum in hadronic collisions," Phys. Rev., vol. C94, no. 2, p. 024917, 2016.

[81] F. O. Durães, A. V. Giannini, V. P. Goncalves, and F. S. Navarra, "Testing the running coupling $k_{T}$-factorization formula for the inclusive gluon production," Phys. Rev., vol. D94, no. 5, p. 054023, 2016.

[82] A. Dumitru and A. V. Giannini, "Initial state angular asymmetries in high energy p+A collisions: spontaneous breaking of rotational symmetry by a color electric field and C-odd fluctuations," Nucl. Phys., vol. A933, pp. 212-228, 2015.

[83] R. P. Feynman, "Very high-energy collisions of hadrons," Phys. Rev. Lett., vol. 23, pp. 1415-1417, 1969.

[84] F. Halzen and A. D. Martin, QUARKS AND LEPTONS: AN INTRODUCTORY COURSE IN MODERN PARTICLE PHYSICS. 1984.

[85] R. P. Feynman, "Photon-hadron interactions," 1973.

[86] H. Fritzsch, M. Gell-Mann, and H. Leutwyler, "Advantages of the Color Octet Gluon Picture," Phys. Lett., vol. B47, pp. 365-368, 1973.

[87] J. D. Bjorken, "THEORETICAL IDEAS ON HIGH-ENERGY INELASTIC ELECTRON - PROTON SCATTERING," in In *Bjorken, J.D.: A collection of summary talks in high energy physics* 41-51, 1969.

[88] J. D. Bjorken, "Asymptotic Sum Rules at Infinite Momentum," Phys. Rev., vol. 179, pp. 1547-1553, 1969.

[89] E. D. Bloom et al., "High-Energy Inelastic e p Scattering at 6-Degrees and 10Degrees," Phys. Rev. Lett., vol. 23, pp. 930-934, 1969.

[90] C. G. Callan, Jr. and D. J. Gross, "High-energy electroproduction and the constitution of the electric current," Phys. Rev. Lett., vol. 22, pp. 156-159, 1969. 
[91] H. L. Lai, J. Huston, S. Kuhlmann, J. Morfin, F. I. Olness, J. F. Owens, J. Pumplin, and W. K. Tung, "Global QCD analysis of parton structure of the nucleon: CTEQ5 parton distributions," Eur. Phys. J., vol. C12, pp. 375-392, 2000.

[92] J. Pumplin, D. R. Stump, J. Huston, H. L. Lai, P. M. Nadolsky, and W. K. Tung, "New generation of parton distributions with uncertainties from global QCD analysis," JHEP, vol. 07, p. 012, 2002.

[93] F. E. Close, An Introduction to Quarks and Partons. 1979.

[94] G. Miller et al., "Inelastic electron-Proton Scattering at Large Momentum Transfers," Phys. Rev., vol. D5, p. 528, 1972.

[95] G. M. Prosperi, M. Raciti, and C. Simolo, "On the running coupling constant in QCD," Prog. Part. Nucl. Phys., vol. 58, pp. 387-438, 2007.

[96] T. van Ritbergen, J. A. M. Vermaseren, and S. A. Larin, "The Four loop beta function in quantum chromodynamics," Phys. Lett., vol. B400, pp. 379-384, 1997.

[97] P. Colangelo and A. Khodjamirian, "QCD sum rules, a modern perspective," 2000 .

[98] R. Gupta, "Introduction to lattice QCD: Course," in Probing the standard model of particle interactions. Proceedings, Summer School in Theoretical Physics, NATO Advanced Study Institute, 68th session, Les Houches, France, July 28September 5, 1997. Pt. 1, 2, pp. 83-219, 1997.

[99] G. P. Lepage, "Lattice QCD for novices," in Strong interactions at low and intermediate energies. Proceedings, 13th Annual Hampton University Graduate Studies, HUGS'98, Newport News, USA, May 26-June 12, 1998, pp. 49-90, 1998.

[100] T. Kucs, QCD resummation techniques. PhD thesis, SUNY, Stony Brook, 2004. 
[101] V. Barone and E. Predazzi, High-Energy Particle Diffraction, vol. v.565 of Texts and Monographs in Physics. Berlin Heidelberg: Springer-Verlag, 2002.

[102] V. D. Barger and R. J. N. Phillips, COLLIDER PHYSICS. 1987.

[103] C. Targett-Adams, "Structure functions and parton distribution functions at the HERA ep collider," Proceedings, 40th Rencontres de Moriond on QCD and High Energy Hadronic Interactions: La Thuile, Aosta Valley, Italy, March 12-19, pp. 227-232, 2005.

[104] M. Gluck, E. Reya, and A. Vogt, "Dynamical parton distributions revisited," Eur. Phys. J., vol. C5, pp. 461-470, 1998.

[105] A. D. Martin, R. G. Roberts, W. J. Stirling, and R. S. Thorne, "NNLO global parton analysis," Phys. Lett., vol. B531, pp. 216-224, 2002.

[106] Y. V. Kovchegov and E. Levin, Quantum chromodynamics at high energy, vol. 33. Cambridge University Press, 2012.

[107] M. Froissart, "Asymptotic behavior and subtractions in the Mandelstam representation," Phys. Rev., vol. 123, pp. 1053-1057, 1961.

[108] S. Chekanov et al., "A ZEUS next-to-leading-order QCD analysis of data on deep inelastic scattering," Phys. Rev., vol. D67, p. 012007, 2003.

[109] Y. Li and K. Tuchin, "Gluon recombination in high parton density QCD: Inclusive pion production," Phys. Rev., vol. D75, p. 074022, 2007.

[110] J. R. Forshaw, M. H. Seymour, and A. Siodmok, "On the Breaking of Collinear Factorization in QCD," JHEP, vol. 11, p. 066, 2012.

[111] J. C. Collins, D. E. Soper, and G. F. Sterman, "Factorization of Hard Processes in QCD," Adv. Ser. Direct. High Energy Phys., vol. 5, pp. 1-91, 1989. 
[112] J.-P. Bouchaud, V. Dupuis, J. Hammann, and E. Vincent, "Separation of time and length scales in spin glasses: Temperature as a microscope," Phys. Rev. B, vol. 65, p. 024439, Dec 2001.

[113] K. J. Golec-Biernat and M. Wusthoff, "Saturation in diffractive deep inelastic scattering," Phys. Rev., vol. D60, p. 114023, 1999.

[114] I. Arsene et al., "Quark gluon plasma and color glass condensate at RHIC? The Perspective from the BRAHMS experiment," Nucl. Phys., vol. A757, pp. 1-27, 2005.

[115] D. Kharzeev, E. Levin, and L. McLerran, "Parton saturation and N(part) scaling of semihard processes in QCD," Phys. Lett., vol. B561, pp. 93-101, 2003.

[116] "Cern announces lhc restart schedule." http://home.web.cern.ch/about/ updates/2014/06/cern-announces-lhc-restart-schedule. Accessed: 201704-10.

[117] R. Pasechnik and M. umbera, "Phenomenological Review on Quark-Gluon Plasma: Concepts vs. Observations," Universe, vol. 3, no. 1, p. 7, 2017.

[118] A. Accardi et al., "Electron Ion Collider: The Next QCD Frontier," Eur. Phys. J., vol. A52, no. 9, p. 268, 2016.

[119] D. Boer et al., "Gluons and the quark sea at high energies: Distributions, polarization, tomography," 2011.

[120] J. L. Abelleira Fernandez et al., "A Large Hadron Electron Collider at CERN: Report on the Physics and Design Concepts for Machine and Detector," J. Phys., vol. G39, p. 075001, 2012.

[121] A. M. Stasto, K. J. Golec-Biernat, and J. Kwiecinski, "Geometric scaling for the total gamma* p cross-section in the low x region," Phys. Rev. Lett., vol. 86, pp. 596-599, 2001. 
[122] C. Marquet and L. Schoeffel, "Geometric scaling in diffractive deep inelastic scattering," Phys. Lett., vol. B639, pp. 471-477, 2006.

[123] M. Derrick et al., "Extraction of the gluon density of the proton at small x," Phys. Lett., vol. B345, pp. 576-588, 1995.

[124] F. Gelis, R. B. Peschanski, G. Soyez, and L. Schoeffel, "Systematics of geometric scaling," Phys. Lett., vol. B647, pp. 376-379, 2007.

[125] G. Altarelli, R. D. Ball, and S. Forte, "An Anomalous dimension for small x evolution," Nucl. Phys., vol. B674, pp. 459-483, 2003.

[126] A. Freund, K. Rummukainen, H. Weigert, and A. Schafer, "Geometric scaling in inclusive eA reactions and nonlinear perturbative QCD," Phys. Rev. Lett., vol. 90, p. 222002, 2003.

[127] E. Iancu, K. Itakura, and L. McLerran, "Geometric scaling above the saturation scale," Nucl. Phys., vol. A708, pp. 327-352, 2002.

[128] L. McLerran and M. Praszalowicz, "Saturation and Scaling of Multiplicity, Mean $p_{T}$ and $p_{T}$ Distributions from $200 \mathrm{GeV} i \sqrt{s}$ i $7 \mathrm{TeV}$," Acta Phys. Polon., vol. B41, pp. 1917-1926, 2010.

[129] L. McLerran and M. Praszalowicz, "Saturation and Scaling of Multiplicity, Mean $p_{T}$ and $p_{T}$ Distributions from $200 \mathrm{GeV}$ i sqrts i $7 \mathrm{TeV}$ - Addendum," Acta Phys. Polon., vol. B42, pp. 99-103, 2011.

[130] M. Praszalowicz, "Geometrical Scaling in Hadronic Collisions," Acta Phys. Polon., vol. B42, pp. 1557-1566, 2011.

[131] L. D. McLerran and R. Venugopalan, "Gluon distribution functions for very large nuclei at small transverse momentum," Phys. Rev., vol. D49, pp. 3352$3355,1994$. 
[132] L. D. McLerran and R. Venugopalan, "Green's functions in the color field of a large nucleus," Phys. Rev., vol. D50, pp. 2225-2233, 1994.

[133] Y. V. Kovchegov, "NonAbelian Weizsacker-Williams field and a two-dimensional effective color charge density for a very large nucleus," Phys. Rev., vol. D54, pp. 5463-5469, 1996.

[134] E. Levin and A. H. Rezaeian, "Gluon saturation and energy dependence of hadron multiplicity in pp and AA collisions at the LHC," Phys. Rev., vol. D83, p. 114001, 2011.

[135] J. L. ALbacete and A. Dumitru, "A model for gluon production in heavy-ion collisions at the LHC with rcBK unintegrated gluon densities," 2010.

[136] K. Aamodt et al., "Centrality dependence of the charged-particle multiplicity density at mid-rapidity in $\mathrm{Pb}-\mathrm{Pb}$ collisions at $\sqrt{s_{N N}}=2.76 \mathrm{TeV}, "$ Phys. Rev. Lett., vol. 106, p. 032301, 2011.

[137] B. I. Abelev et al., "Long range rapidity correlations and jet production in high energy nuclear collisions," Phys. Rev., vol. C80, p. 064912, 2009.

[138] G. Aad et al., "Measurement of the azimuthal anisotropy for charged particle production in $\sqrt{s_{N N}}=2.76 \mathrm{TeV}$ lead-lead collisions with the ATLAS detector," Phys. Rev., vol. C86, p. 014907, 2012.

[139] S. Chatrchyan et al., "Centrality dependence of dihadron correlations and azimuthal anisotropy harmonics in $\mathrm{PbPb}$ collisions at $\sqrt{s_{N N}}=2.76 \mathrm{TeV}$," Eur. Phys. J., vol. C72, p. 2012, 2012.

[140] A. Dumitru, F. Gelis, L. McLerran, and R. Venugopalan, "Glasma flux tubes and the near side ridge phenomenon at RHIC," Nucl. Phys., vol. A810, pp. 91-108, 2008 . 
[141] P. Bozek, "Collective flow in p-Pb and d-Pd collisions at TeV energies," Phys. Rev., vol. C85, p. 014911, 2012.

[142] P. Bozek and W. Broniowski, "Correlations from hydrodynamic flow in p-Pb collisions," Phys. Lett., vol. B718, pp. 1557-1561, 2013.

[143] P. Bozek and W. Broniowski, "Collective dynamics in high-energy protonnucleus collisions," Phys. Rev., vol. C88, no. 1, p. 014903, 2013.

[144] A. Bzdak, B. Schenke, P. Tribedy, and R. Venugopalan, "Initial state geometry and the role of hydrodynamics in proton-proton, proton-nucleus and deuteronnucleus collisions," Phys. Rev., vol. C87, no. 6, p. 064906, 2013.

[145] E. Shuryak and I. Zahed, "High-multiplicity pp and pA collisions: Hydrodynamics at its edge," Phys. Rev., vol. C88, no. 4, p. 044915, 2013.

[146] P. Bozek, W. Broniowski, and G. Torrieri, "Mass hierarchy in identified particle distributions in proton-lead collisions," Phys. Rev. Lett., vol. 111, p. 172303, 2013.

[147] K. Werner, M. Bleicher, B. Guiot, I. Karpenko, and T. Pierog, "Evidence for Flow from Hydrodynamic Simulations of $p$ - $\mathrm{Pb}$ Collisions at $5.02 \mathrm{TeV}$ from $\nu_{2}$ Mass Splitting," Phys. Rev. Lett., vol. 112, no. 23, p. 232301, 2014.

[148] B. Schenke and R. Venugopalan, "Eccentric protons? Sensitivity of flow to system size and shape in $\mathrm{p}+\mathrm{p}, \mathrm{p}+\mathrm{Pb}$ and $\mathrm{Pb}+\mathrm{Pb}$ collisions," Phys. Rev. Lett., vol. 113, p. 102301, 2014.

[149] R. Snellings, "Elliptic Flow: A Brief Review," New J. Phys., vol. 13, p. 055008, 2011.

[150] S. A. Voloshin, A. M. Poskanzer, and R. Snellings, "Collective phenomena in non-central nuclear collisions," 2008. 
[151] V. Khachatryan et al., "Observation of Long-Range Near-Side Angular Correlations in Proton-Proton Collisions at the LHC," JHEP, vol. 09, p. 091, 2010.

[152] G. Aad et al., "Observation of Long-Range Elliptic Azimuthal Anisotropies in $\sqrt{s}=13$ and $2.76 \mathrm{TeV} p p$ Collisions with the ATLAS Detector," Phys. Rev. Lett., vol. 116, no. 17, p. 172301, 2016.

[153] S. Chatrchyan et al., "Observation of long-range near-side angular correlations in proton-lead collisions at the LHC," Phys. Lett., vol. B718, pp. 795-814, 2013.

[154] G. Aad et al., "Measurement of long-range pseudorapidity correlations and azimuthal harmonics in $\sqrt{s_{N N}}=5.02 \mathrm{TeV}$ proton-lead collisions with the ATLAS detector," Phys. Rev., vol. C90, no. 4, p. 044906, 2014.

[155] K. Werner, I. Karpenko, T. Pierog, M. Bleicher, and K. Mikhailov, "Evidence for hydrodynamic evolution in proton-proton scattering at $900 \mathrm{GeV}$," Phys. Rev., vol. C83, p. 044915, 2011.

[156] K. Werner, I. Karpenko, and T. Pierog, "The 'Ridge' in Proton-Proton Scattering at 7 TeV," Phys. Rev. Lett., vol. 106, p. 122004, 2011.

[157] C. Shen, J.-F. Paquet, G. S. Denicol, S. Jeon, and C. Gale, "Collectivity and electromagnetic radiation in small systems," Phys. Rev., vol. C95, no. 1, p. 014906 , 2017.

[158] P. Romatschke, "Light-Heavy Ion Collisions: A window into pre-equilibrium QCD dynamics?," Eur. Phys. J., vol. C75, no. 7, p. 305, 2015.

[159] I. Kozlov, M. Luzum, G. Denicol, S. Jeon, and C. Gale, "Transverse momentum structure of pair correlations as a signature of collective behavior in small collision systems," 2014. 
[160] A. Dumitru, K. Dusling, F. Gelis, J. Jalilian-Marian, T. Lappi, and R. Venugopalan, "The Ridge in proton-proton collisions at the LHC," Phys. Lett., vol. B697, pp. 21-25, 2011.

[161] K. Dusling and R. Venugopalan, "Azimuthal collimation of long range rapidity correlations by strong color fields in high multiplicity hadron-hadron collisions," Phys. Rev. Lett., vol. 108, p. 262001, 2012.

[162] K. Dusling and R. Venugopalan, "Explanation of systematics of CMS p+Pb high multiplicity di-hadron data at $\sqrt{s}_{\mathrm{NN}}=5.02 \mathrm{TeV}$," Phys. Rev., vol. D87, no. 5, p. $054014,2013$.

[163] K. Dusling and R. Venugopalan, "Comparison of the color glass condensate to dihadron correlations in proton-proton and proton-nucleus collisions," Phys. Rev., vol. D87, no. 9, p. 094034, 2013.

[164] A. Dumitru, D. E. Kharzeev, E. M. Levin, and Y. Nara, "Gluon Saturation in $p A$ Collisions at the LHC: KLN Model Predictions For Hadron Multiplicities," Phys. Rev., vol. C85, p. 044920, 2012.

[165] A. Dumitru, A. V. Giannini, and V. Skokov, "Anisotropic particle production and azimuthal correlations in high-energy pA collisions," arXiv:1503.03897, 2015 .

[166] J. L. Albacete, A. Dumitru, H. Fujii, and Y. Nara, "CGC predictions for p + Pb collisions at the LHC," Nucl. Phys., vol. A897, pp. 1-27, 2013.

[167] S. Gavin, L. McLerran, and G. Moschelli, "Long Range Correlations and the Soft Ridge in Relativistic Nuclear Collisions," Phys. Rev., vol. C79, p. 051902, 2009 .

[168] A. Dumitru and J. Jalilian-Marian, "Two-particle correlations in high energy collisions and the gluon four-point function," Phys. Rev., vol. D81, p. 094015, 2010. 
[169] A. Kovner and M. Lublinsky, "Angular Correlations in Gluon Production at High Energy," Phys. Rev., vol. D83, p. 034017, 2011.

[170] A. Kovner and M. Lublinsky, "On Angular Correlations and High Energy Evolution," Phys. Rev., vol. D84, p. 094011, 2011.

[171] E. Levin and A. H. Rezaeian, "The Ridge from the BFKL evolution and beyond," Phys. Rev., vol. D84, p. 034031, 2011.

[172] E. Iancu and D. N. Triantafyllopoulos, "Higher-point correlations from the JIMWLK evolution," JHEP, vol. 11, p. 105, 2011.

[173] Y. V. Kovchegov and D. E. Wertepny, "Two-Gluon Correlations in Heavy-Light Ion Collisions: Energy and Geometry Dependence, IR Divergences, and $k_{T^{-}}$ Factorization," Nucl. Phys., vol. A925, pp. 254-295, 2014.

[174] R. L. Ray, "Phenomenological analysis of angular correlations in $7 \mathrm{TeV}$ protonproton collisions from the CMS experiment," Phys. Rev., vol. D84, p. 034020, 2011.

[175] R. L. Ray, "Azimuthal quadrupole correlation from gluon interference in 200 GeV and 7 TeV p+p collisions," Phys. Rev., vol. D90, no. 5, p. 054013, 2014.

[176] A. Dumitru, L. McLerran, and V. Skokov, "Azimuthal asymmetries and the emergence of collectivity from multi-particle correlations in high-energy pA collisions," Phys. Lett., vol. B743, pp. 134-137, 2015.

[177] A. Kovner and A. H. Rezaeian, "Diphoton ridge in $\mathrm{p}+\mathrm{p}$ and $\mathrm{p}+\mathrm{A}$ collisions at RHIC and the LHC," Phys. Rev., vol. D92, no. 7, p. 074045, 2015.

[178] J.-Y. Ollitrault, "Anisotropy as a signature of transverse collective flow," Phys. Rev., vol. D46, pp. 229-245, 1992.

[179] B. Abelev et al., "Long-range angular correlations on the near and away side in p-Pb collisions at $\sqrt{s_{N N}}=5.02 \mathrm{TeV}, "$ Phys. Lett., vol. B719, pp. 29-41, 2013. 
[180] B. B. Abelev et al., "Multiparticle azimuthal correlations in $\mathrm{p}-\mathrm{Pb}$ and $\mathrm{Pb}-\mathrm{Pb}$ collisions at the CERN Large Hadron Collider," Phys. Rev., vol. C90, no. 5, p. $054901,2014$.

[181] G. Aad et al., "Observation of Associated Near-Side and Away-Side Long-Range Correlations in $\sqrt{s_{N N}}=5.02$ ??TeV Proton-Lead Collisions with the ATLAS Detector," Phys. Rev. Lett., vol. 110, no. 18, p. 182302, 2013.

[182] G. Aad et al., "Measurement with the ATLAS detector of multi-particle azimuthal correlations in $\mathrm{p}+\mathrm{Pb}$ collisions at $\sqrt{s_{N N}}=5.02 \mathrm{TeV}, "$ Phys. Lett., vol. B725, pp. 60-78, 2013.

[183] T. A. collaboration, "Measurement of long-range pseudorapidity correlations and azimuthal harmonics in $\sqrt{s_{\mathrm{NN}}}=5.02 \mathrm{TeV}$ proton-lead collisions with the ATLAS detector," 2014.

[184] S. Chatrchyan et al., "Multiplicity and transverse momentum dependence of two- and four-particle correlations in $\mathrm{pPb}$ and $\mathrm{PbPb}$ collisions," Phys. Lett., vol. B724, pp. 213-240, 2013.

[185] A. Adare et al., "Quadrupole Anisotropy in Dihadron Azimuthal Correlations in Central $d+\mathrm{Au}$ Collisions at $\sqrt{s_{N N}}=200 \mathrm{GeV}$," Phys. Rev. Lett., vol. 111, no. 21, p. 212301, 2013.

[186] R. Venugopalan, "Introduction to light cone field theory and high-energy scattering," 1998. [Lect. Notes Phys.516,89(1999)].

[187] A. H. Mueller, "Parton saturation: An Overview," in QCD perspectives on hot and dense matter. Proceedings, NATO Advanced Study Institute, Summer School, Cargese, France, August 6-18, 2001, pp. 45-72, 2001.

[188] H. Kowalski, L. Motyka, and G. Watt, "Exclusive diffractive processes at HERA within the dipole picture," Phys. Rev., vol. D74, p. 074016, 2006. 
[189] E. Gotsman, M. Kozlov, E. Levin, U. Maor, and E. Naftali, "Towards a new global QCD analysis: Solution to the nonlinear equation at arbitrary impact parameter," Nucl. Phys., vol. A742, pp. 55-79, 2004.

[190] K. J. Golec-Biernat and A. M. Stasto, "On solutions of the Balitsky-Kovchegov equation with impact parameter," Nucl. Phys., vol. B668, pp. 345-363, 2003.

[191] A. H. Mueller, "Small x Behavior and Parton Saturation: A QCD Model," Nucl. Phys., vol. B335, pp. 115-137, 1990.

[192] R. J. Glauber, "Cross-sections in deuterium at high-energies," Phys. Rev., vol. 100, pp. 242-248, 1955.

[193] R. J. Glauber and G. Matthiae, "High-energy scattering of protons by nuclei," Nucl. Phys., vol. B21, pp. 135-157, 1970.

[194] I. Balitsky, "High-energy QCD and Wilson lines," 2001.

[195] M. E. Peskin and D. V. Schroeder, An Introduction to quantum field theory. 1995.

[196] A. Dumitru and J. Jalilian-Marian, "Forward quark jets from protons shattering the colored glass," Phys. Rev. Lett., vol. 89, p. 022301, 2002.

[197] F. Gelis and J. Jalilian-Marian, "From DIS to proton nucleus collisions in the color glass condensate model," Phys. Rev., vol. D67, p. 074019, 2003.

[198] E. Wessels, Signatures of gluon saturation in high energy scattering. PhD thesis, Vrije U., 2009.

[199] M. S. Kugeratski, V. P. Goncalves, and F. S. Navarra, "Testing universality of the color glass condensate descriptions," Eur. Phys. J., vol. C44, pp. 577-586, 2005. 
[200] J. L. Albacete, N. Armesto, J. G. Milhano, and C. A. Salgado, "Non-linear QCD meets data: A Global analysis of lepton-proton scattering with running coupling BK evolution," Phys. Rev., vol. D80, p. 034031, 2009.

[201] D. Boer, A. Utermann, and E. Wessels, "Compatibility of phenomenological dipole cross sections with the Balitsky-Kovchegov equation," Phys. Rev., vol. D75, p. $094022,2007$.

[202] K. Kutak, "Saturation and linear transport equation," Phys. Lett., vol. B675, pp. 332-335, 2009.

[203] M. A. Betemps, V. P. Goncalves, and J. T. de Santana Amaral, "Testing Nonlinear Evolution with Running Coupling Corrections in ep and pp collisions," Eur. Phys. J., vol. C66, pp. 137-146, 2010.

[204] A. Dumitru, A. Hayashigaki, and J. Jalilian-Marian, "Geometric scaling violations in the central rapidity region of d + Au collisions at RHIC," Nucl. Phys., vol. A770, pp. 57-70, 2006.

[205] D. Boer, A. Utermann, and E. Wessels, "Geometric Scaling at RHIC and LHC," Phys. Rev., vol. D77, p. 054014, 2008.

[206] N. Armesto, "Introduction to low x physics and saturation," Acta Phys. Polon., vol. B35, pp. 213-222, 2004.

[207] C. Albajar et al., "Production of Low Transverse Energy Clusters in anti-p p Collisions at $\mathrm{s}^{* *}(1 / 2)=0.2-\mathrm{TeV}$ to $0.9-\mathrm{TeV}$ and their Interpretation in Terms of QCD Jets," Nucl. Phys., vol. B309, pp. 405-425, 1988.

[208] F. Ceradini, "STUDY OF MINIMUM BIAS TRIGGER EVENTS AT s**(1/2) $=0.2-\mathrm{TeV}$ TO 0.9-TeV WITH MAGNETIC AND CALORIMETRIC ANALYSIS AT THE CERN PROTON - ANTI-PROTON COLlider," Conf. Proc., vol. C850718, p. 363, 1985. 
[209] I. Sarcevic, S. D. Ellis, and P. Carruthers, "QCD MINIJET CROSSSECTIONS," Phys. Rev., vol. D40, p. 1446, 1989.

[210] T. K. Gaisser and F. Halzen, "Soft Hard Scattering in the TeV Range," Phys. Rev. Lett., vol. 54, p. 1754, 1985.

[211] E. Eichten, I. Hinchliffe, K. D. Lane, and C. Quigg, "Super Collider Physics," Rev. Mod. Phys., vol. 56, pp. 579-707, 1984. [Addendum: Rev. Mod. Phys.58,1065(1986)].

[212] F. Cooper, E. Mottola, and G. C. Nayak, "Minijet initial conditions for nonequilibrium parton evolution at RHIC and LHC," Phys. Lett., vol. B555, pp. 181-188, 2003.

[213] X.-N. Wang, "pQCD based approach to parton production and equilibration in high-energy nuclear collisions," Phys. Rept., vol. 280, pp. 287-371, 1997.

[214] B. L. Combridge, J. Kripfganz, and J. Ranft, "Hadron Production at Large Transverse Momentum and QCD," Phys. Lett., vol. 70B, p. 234, 1977.

[215] H. G. Dosch, F. S. Navarra, M. Nielsen, and M. Rueter, "Charmonium hadron cross-section in a nonperturbative QCD approach," Phys. Lett., vol. B466, pp. 363-368, 1999.

[216] K. A. Olive et al., "Review of Particle Physics," Chin. Phys., vol. C38, p. 090001, 2014 .

[217] G. Antchev et al., "Luminosity-independent measurements of total, elastic and inelastic cross-sections at $\sqrt{s}=7 \mathrm{TeV}$," Europhys. Lett., vol. 101, p. 21004, 2013.

[218] G. Antchev et al., "Luminosity-Independent Measurement of the Proton-Proton Total Cross Section at $\sqrt{s}=8 \mathrm{TeV}, "$ Phys. Rev. Lett., vol. 111, no. 1, p. 012001, 2013. 
[219] K. Belov, "Proton-air Inelastic Cross-Section Measurement at Ultra-High Energies by HiRes," in Proceedings, 30th International Cosmic Ray Conference (ICRC 2007): Merida, Yucatan, Mexico, July 3-11, 2007, vol. 4, pp. 687-690, 2007.

[220] P. Abreu et al., "Measurement of the proton-air cross-section at $\sqrt{s}=57 \mathrm{TeV}$ with the Pierre Auger Observatory," Phys. Rev. Lett., vol. 109, p. 062002, 2012.

[221] L. Durand and H. Pi, "QCD and Rising Total Cross-Sections," Phys. Rev. Lett., vol. 58, pp. 303-306, 1987.

[222] R. M. Godbole, A. Grau, G. Pancheri, and Y. N. Srivastava, "Minijets, soft gluon resummation and photon cross-sections," Nucl. Phys. Proc. Suppl., vol. 184, pp. 85-90, 2008.

[223] R. M. Godbole, A. Grau, R. Hegde, G. Pancheri, and Y. Srivastava, "Theoretical expectations for total cross-sections at the large hadron collider," Pramana, vol. 66, pp. 657-668, 2006.

[224] M. M. Block, "Hadronic forward scattering: Predictions for the Large Hadron Collider and cosmic rays," Phys. Rept., vol. 436, pp. 71-215, 2006.

[225] Y. Afek, C. Leroy, B. Margolis, and P. Valin, "Differential and Total Proton Cross-sections, Particle Production and the Parton Model," Phys. Rev. Lett., vol. 45 , p. 85,1980 .

[226] P. L'Heureux, B. Margolis, and P. Valin, "QUARK - GLUON MODEL FOR DIFFRACTION AT HIGH-ENERGIES," Phys. Rev., vol. D32, pp. 1681-1691, 1985 .

[227] L. Durand and H. Pi, "High-energy Nucleon Nucleus Scattering and Cosmic Ray Cross-sections," Phys. Rev., vol. D38, pp. 78-84, 1988. 
[228] L. Durand and H. Pi, "Semihard QCD and High-energy $p p$ and $\bar{p} p$ Scattering," Phys. Rev., vol. D40, p. 1436, 1989.

[229] B. Margolis, P. Valin, M. M. Block, F. Halzen, and R. S. Fletcher, "Forward Scattering Amplitudes in Semihard QCD," Phys. Lett., vol. B213, p. 221, 1988.

[230] X.-N. Wang, "Role of multiple mini - jets in high-energy hadronic reactions," Phys. Rev., vol. D43, pp. 104-112, 1991.

[231] F. Carvalho, F. O. Duraes, and E. G. S. Luna, "Gluon saturation and proton anti-proton cross sections," Braz. J. Phys., vol. 37, pp. 110-113, 2007.

[232] F. O. Duraes, V. P. Goncalves, F. S. Navarra, A. L. V. R. dos Reis, and G. Wilk, "Gluon saturation and leading particle spectra in p p collisions," Braz. J. Phys., vol. 37, pp. 122-125, 2007.

[233] F. O. Duraes, "J/psi production in the saturation regime," Braz. J. Phys., vol. 37, pp. 114-117, 2007.

[234] F. Carvalho, F. O. Duraes, F. S. Navarra, and S. Szpigel, "Charm and longitudinal structure functions with the KLN model," Phys. Rev., vol. C79, p. 035211, 2009.

[235] A. Capella and J. Tran Thanh Van, "High Order Moments of the $\bar{p} p$ Multiplicity Distribution in the Dual Parton Model," Z. Phys., vol. C23, p. 165, 1984. [Erratum: Z. Phys.C25,102(1984)].

[236] J. Dias de Deus and J. Kwiecinski, "Semihard QCD: Mini - Jets and Elastic Scattering," Phys. Lett., vol. B196, pp. 537-542, 1987.

[237] R. C. Hwa, "Geometrical Branching Model: Correlations and Jets," Phys. Rev., vol. D37, p. 1830, 1988.

[238] R. J. Glauber, "HIGH-ENERGY COLLISION THEORY," In *Lo, S. Y. (ed.): Geometrical pictures in hadronic collisions*, 83-182, 1987. 
[239] M. M. Islam, "Impact Parameter Representation from Sommerfeld-Watson Transform," Nucl. Phys., vol. B104, pp. 511-532, 1976.

[240] J. Sakurai and J. Napolitano, Modern Quantum Mechanics. Addison-Wesley, 2011.

[241] C. Joachain, Quantum collision theory. North-Holland, 1975.

[242] M. M. Block and R. N. Cahn, "High-Energy $p \bar{p}$ and $p p$ Forward Elastic Scattering and Total Cross-Sections," Rev. Mod. Phys., vol. 57, p. 563, 1985.

[243] V. V. Anisovich, M. N. Kobrinsky, J. Nyiri, and Yu. M. Shabelski, QUARK MODEL AND HIGH-ENERGY COLLISIONS. 1986.

[244] R. Ulrich, R. Engel, S. Muller, F. Schussler, and M. Unger, "Proton-Air Cross Section and Extensive Air Showers," Nucl. Phys. Proc. Suppl., vol. 196, pp. 335$340,2009$.

[245] M. M. Block, E. M. Gregores, F. Halzen, and G. Pancheri, "Photon - proton and photon-photon scattering from nucleon-nucleon forward amplitudes," Phys. Rev., vol. D60, p. 054024, 1999.

[246] E. G. S. Luna, "Diffraction and an infrared finite gluon propagator," Braz. J. Phys., vol. 37, pp. 84-87, 2007.

[247] E. G. S. Luna and A. A. Natale, "Gamma p and gamma gamma scattering from anti-p p, pp forward amplitudes in a QCD Eikonal model with a dynamical gluon mass," Phys. Rev., vol. D73, p. 074019, 2006.

[248] A. Achilli, R. M. Godbole, A. Grau, G. Pancheri, O. Shekhovtsova, and Y. N. Srivastava, "Total and inelastic cross-sections at LHC at sqrts $=7-\mathrm{TeV}$ and beyond," Phys. Rev., vol. D84, p. 094009, 2011. 
[249] A. Grau, G. Pancheri, O. Shekhovtsova, and Y. N. Srivastava, "Modeling pion and proton total cross-sections at LHC," Phys. Lett., vol. B693, pp. 456-461, 2010 .

[250] R. M. Godbole, A. Grau, G. Pancheri, and Y. N. Srivastava, "Soft gluons and the energy dependence of total cross-sections," in Proceedings, 1st International Workshop on QCD - Theory and Experiment (QCD@Work 2001): Martina Franca, Bari, Italy, June 16-20, 2001, pp. 127-138, 2002.

[251] R. M. Godbole, A. Grau, and G. Pancheri, "Models for photon-photon total cross-sections," Nucl. Phys. Proc. Suppl., vol. 82, pp. 246-251, 2000.

[252] P. Lipari and M. Lusignoli, "Multiple Parton Interactions in Hadron Collisions and Diffraction," Phys. Rev., vol. D80, p. 074014, 2009.

[253] A. Grau, G. Pancheri, and Y. N. Srivastava, "Hadronic total cross-sections through soft gluon summation in impact parameter space," Phys. Rev., vol. D60, p. $114020,1999$.

[254] M. M. Block, F. Halzen, and B. Margolis, "How large is the total cross-section at supercollider energies?," Phys. Rev., vol. D45, pp. 839-843, 1992.

[255] R. M. Godbole, A. Grau, G. Pancheri, and Y. N. Srivastava, "Soft gluon radiation and energy dependence of total hadronic cross-sections," Phys. Rev., vol. D72, p. 076001, 2005.

[256] M. M. Block and F. Halzen, "Forward hadronic scattering at $8 \mathrm{TeV}$ : predictions for the LHC," Phys. Rev., vol. D86, p. 014006, 2012.

[257] M. J. Menon and P. V. R. G. Silva, "A study on analytic parametrizations for protonproton cross-sections and asymptotia," J. Phys., vol. G40, p. 125001, 2013. [Erratum: J. Phys.G41,019501(2014)]. 
[258] B. Abelev et al., "Measurement of inelastic, single- and double-diffraction cross sections in proton-proton collisions at the LHC with ALICE," Eur. Phys. J., vol. C73, no. 6, p. 2456, 2013.

[259] G. Aad et al., "Measurement of the Inelastic Proton-Proton Cross-Section at $\sqrt{s}=7 \mathrm{TeV}$ with the ATLAS Detector," Nature Commun., vol. 2, p. 463, 2011.

[260] S. Chatrchyan et al., "Measurement of the inelastic proton-proton cross section at $\sqrt{s}=7$ TeV," Phys. Lett., vol. B722, pp. 5-27, 2013.

[261] E. G. S. Luna, A. F. Martini, M. J. Menon, A. Mihara, and A. A. Natale, "Influence of a dynamical gluon mass in the pp and p anti-p forward scattering," Phys. Rev., vol. D72, p. 034019, 2005.

[262] P. Lipari and M. Lusignoli, "Interpretation of the measurements of total, elastic and diffractive cross sections at LHC," Eur. Phys. J., vol. C73, no. 11, p. 2630, 2013.

[263] B. Alessandro et al., "Hadron-Hadron and Cosmic-Ray Interactions at multi-TeV Energies," arXiv:1101.1852, 2011.

[264] D. d'Enterria, R. Engel, T. Pierog, S. Ostapchenko, and K. Werner, "The Strong interaction at the collider and cosmic-rays frontiers," Few Body Syst., vol. 53, pp. 173-179, 2012.

[265] D. d'Enterria, R. Engel, T. Pierog, S. Ostapchenko, and K. Werner, "Constraints from the first LHC data on hadronic event generators for ultra-high energy cosmic-ray physics," Astropart. Phys., vol. 35, pp. 98-113, 2011.

[266] M. Grothe, F. Hautmann, and S. Ostapchenko, "Introduction to Forward Physics and Cosmic Rays at ISMD 2010," in Proceedings, 40th International Symposium on Multiparticle Dynamics (ISMD 2010): Antwerp, Belgium, September 21-25, 2010, pp. 213-226, 2011. 
[267] S. Ostapchenko, "On the model dependence of the relation between minimumbias and inelastic proton-proton cross sections," Phys. Lett., vol. B703, pp. 588$592,2011$.

[268] R. Ulrich, R. Engel, and M. Unger, "Hadronic Multiparticle Production at UltraHigh Energies and Extensive Air Showers," Phys. Rev., vol. D83, p. 054026, 2011.

[269] R. Engel, T. K. Gaisser, P. Lipari, and T. Stanev, "Proton proton cross-section at $\sqrt{s}$ similar to 30-TeV," Phys. Rev., vol. D58, p. 014019, 1998.

[270] B. Z. Kopeliovich, "Transparent nuclei and deuteron gold collisions at RHIC," Phys. Rev., vol. C68, p. 044906, 2003.

[271] R. Vogt, "Relation of hard and total cross-sections to centrality," Heavy Ion Phys., vol. 9, pp. 339-348, 1999.

[272] M. Aglietta et al., "Measurement of the proton-air inelastic cross section at s sim Te-2V from the EAS-TOP experiment," Phys. Rev., vol. D79, p. 032004, 2009 .

[273] G. Aielli et al., "Proton-air cross section measurement with the ARGO-YBJ cosmic ray experiment," Phys. Rev., vol. D80, p. 092004, 2009.

[274] R. M. Baltrusaitis, G. L. Cassiday, J. W. Elbert, P. R. Gerhardy, S. Ko, E. C. Loh, Y. Mizumoto, P. Sokolsky, and D. Steck, "Total Proton Proton CrossSection at ${ }^{* *}(1 / 2)=30-\mathrm{TeV}, "$ Phys. Rev. Lett., vol. 52, pp. 1380-1383, 1984.

[275] M. Honda, M. Nagano, S. Tonwar, K. Kasahara, T. Hara, N. Hayashida, Y. Matsubara, M. Teshima, and S. Yoshida, "Inelastic cross-section for p-air collisions from air shower experiment and total cross-section for $\mathrm{p} \mathrm{p}$ collisions at SSC energy," Phys. Rev. Lett., vol. 70, pp. 525-528, 1993.

[276] K. Belov, "p-air cross-section measurement at 10**18.5-eV," Nucl. Phys. Proc. Suppl., vol. 151, pp. 197-204, 2006. [,197(2006)]. 
[277] H. H. Mielke, M. Foeller, J. Engler, and J. Knapp, "Cosmic ray hadron flux at sea level up to 15-TeV," J. Phys., vol. G20, pp. 637-649, 1994.

[278] F. Siohan, R. W. Ellsworth, A. S. Ito, J. R. Macfall, R. E. Streitmatter, S. C. Tonwar, and G. B. Yodh, "Unaccompanied Hadron Flux at a Depth of $730 \mathrm{~g}$ $\mathrm{cm}^{-2}, 10^{2}<E<10^{4} \mathrm{GeV}$," J. Phys., vol. G4, no. 7, pp. 1169-1186, 1978.

[279] I. De Mitri, D. Martello, L. Perrone, A. Surdo, and M. Zha, "Proton-air inelastic cross section measurement with ARGO-YBJ," in Proceedings, 30th International Cosmic Ray Conference (ICRC 2007): Merida, Yucatan, Mexico, July 3-11, 2007, vol. 4, pp. 675-678, 2007.

[280] G. b. Yodh, S. c. Tonwar, T. k. Gaisser, and R. w. Ellsworth, "PROTON PROTON CROSS-SECTIONS FROM 1 TO 100-TeV," Phys. Rev., vol. D27, pp. 1183-1186, 1983.

[281] S. P. Knurenko, V. R. Sleptsova, I. E. Sleptsov, N. N. Kalmykov, and S. S. Ostapchenko, "Longitudinal EAS development at $\mathrm{E}(0)=10^{* *} 18-\mathrm{eV}$ to $3 \times 10^{* *} 19-\mathrm{eV}$ and the QGSJET model," in Proceedings, 26th International Cosmic Ray Conference, August 17-25, 1999, Salt Lake City: Invited, Rapporteur, and Highlight Papers, pp. 372-375, 1999.

[282] N. Cartiglia, "Measurement of the proton-proton total, elastic, inelastic and diffractive cross sections at 2, 7, 8 and $57 \mathrm{TeV}, "$ arXiv:1305.6131.

[283] R. Ulrich, J. Blumer, R. Engel, F. Schussler, and M. Unger, "On the measurement of the proton-air cross section using air shower data," New J. Phys., vol. 11, p. 065018, 2009.

[284] X.-N. Wang and M. Gyulassy, "HIJING: A Monte Carlo model for multiple jet production in p p, p A and A A collisions," Phys. Rev., vol. D44, pp. 3501-3516, 1991. 
[285] S. Chekanov et al., "Measurement of the photon proton total cross-section at a center-of-mass energy of 209-GeV at HERA," Nucl. Phys., vol. B627, pp. 3-28, 2002.

[286] S. Aid et al., "Measurement of the total photon-proton cross-section and its decomposition at 200-GeV center-of-mass energy," Z. Phys., vol. C69, pp. 2738, 1995.

[287] G. M. Vereshkov, O. D. Lalakulich, Yu. F. Novoseltsev, and R. V. Novoseltseva, "Total cross section for photon nucleon interaction in the energy range $\sqrt{s}$ = 40-GeV - 250-GeV," Phys. Atom. Nucl., vol. 66, pp. 565-574, 2003. [Yad. Fiz.66,591(2003)].

[288] G. Abbiendi et al., "Total hadronic cross-section of photon-photon interactions at LEP," Eur. Phys. J., vol. C14, pp. 199-212, 2000.

[289] M. Acciarri et al., "Cross-section of hadron production in gamma gamma collisions at LEP," Phys. Lett., vol. B408, pp. 450-464, 1997.

[290] M. Acciarri et al., "Total cross-section in $\gamma \gamma$ collisions at LEP," Phys. Lett., vol. B519, pp. 33-45, 2001.

[291] H. Aihara et al., "A measurement of the total hadronic cross-section in tagged $\gamma \gamma$ reactions," Phys. Rev., vol. D41, p. 2667, 1990.

[292] S. E. Baru et al., "Total cross-section of two photon production of hadrons," $Z$. Phys., vol. C53, pp. 219-224, 1992.

[293] C. Berger et al., "Measurement of the Total Photon-photon Cross-section for the Production of Hadrons at Small $Q^{2}, "$ Phys. Lett., vol. B149, pp. 421-426, 1984.

[294] C. Berger et al., "A Measurement of the $Q^{2}$ and $W$ Dependence of the $\gamma \gamma$ Total Cross-section for Hadron Production," Z. Phys., vol. C26, p. 353, 1984. 
[295] D. Bintinger et al., "Measurement of the Total Hadronic Cross-section in Photonphoton Interactions," Phys. Rev. Lett., vol. 54, p. 763, 1985.

[296] E. Levin and A. H. Rezaeian, "Gluon saturation and inclusive hadron production at LHC," Phys. Rev., vol. D82, p. 014022, 2010.

[297] B. Abelev et al., "Transverse momentum distribution and nuclear modification factor of charged particles in $p$-Pb collisions at $\sqrt{s_{N N}}=5.02 \mathrm{TeV}$," Phys. Rev. Lett., vol. 110, no. 8, p. 082302, 2013.

[298] Y. Mehtar-Tani and G. Wolschin, "Baryon Stopping as a new Probe of Geometric Scaling," Phys. Rev. Lett., vol. 102, p. 182301, 2009.

[299] J. L. Albacete and C. Marquet, "Single Inclusive Hadron Production at RHIC and the LHC from the Color Glass Condensate," Phys. Lett., vol. B687, pp. 174$179,2010$.

[300] T. Lappi and H. Mntysaari, "Single inclusive particle production at high energy from HERA data to proton-nucleus collisions," Phys. Rev., vol. D88, p. 114020, 2013.

[301] Y. Mehtar-Tani and G. Wolschin, "Baryon stopping and saturation physics in relativistic collisions," Phys. Rev., vol. C80, p. 054905, 2009.

[302] H. Appelshauser et al., "Baryon stopping and charged particle distributions in central $\mathrm{Pb}+\mathrm{Pb}$ collisions at $158-\mathrm{GeV}$ per nucleon," Phys. Rev. Lett., vol. 82, pp. 2471-2475, 1999.

[303] I. G. Bearden et al., "Nuclear stopping in $\mathrm{Au}+\mathrm{Au}$ collisions at $\mathrm{s}(\mathrm{NN})^{* *}(1 / 2)$ = 200-GeV," Phys. Rev. Lett., vol. 93, p. 102301, 2004.

[304] H. H. Dalsgaard, "Nuclear stopping: Paving the way from RHIC to LHC," Int. J. Mod. Phys., vol. E16, pp. 1813-1818, 2007. 
[305] I. C. Arsene et al., "Nuclear stopping and rapidity loss in Au+Au collisions at s(NN)**(1/2) = 62.4-GeV," Phys. Lett., vol. B677, pp. 267-271, 2009.

[306] R. Debbe, "Recent results from BRAHMS," J. Phys., vol. G35, p. 104004, 2008.

[307] F. Videbaek and O. Hansen, "Baryon rapidity loss and mid-rapidity stacking in high-energy nucleus-nucleus collisions," Phys. Rev., vol. C52, pp. 2684-2693, 1995.

[308] L. Ahle et al., "Proton and deuteron production in Au + Au reactions at 11.6/AGeV/c," Phys. Rev., vol. C60, p. 064901, 1999.

[309] B. B. Back et al., "Baryon rapidity loss in relativistic Au+Au collisions," Phys. Rev. Lett., vol. 86, pp. 1970-1973, 2001.

[310] S. Albino, B. A. Kniehl, and G. Kramer, "AKK Update: Improvements from New Theoretical Input and Experimental Data," Nucl. Phys., vol. B803, pp. 42$104,2008$.

[311] B. A. Kniehl, G. Kramer, and B. Potter, "Fragmentation functions for pions, kaons, and protons at next-to-leading order," Nucl. Phys., vol. B582, pp. 514$536,2000$.

[312] B. B. Abelev et al., "Multiplicity Dependence of Pion, Kaon, Proton and Lambda Production in p-Pb Collisions at $\sqrt{s_{N N}}=5.02 \mathrm{TeV}$," Phys. Lett., vol. B728, pp. 25-38, 2014.

[313] T. Sako, "LHCf Measurements of Very Forward Particles at LHC," in Proceedings, 16th International Symposium on Very High Energy Cosmic Ray Interactions (ISVHECRI 2010): Batavia, Illinois, USA, June 28-July 2, 2010, 2010.

[314] O. Adriani et al., "Early results of the LHCf Experiment and their contribution to Ultra-High-Energy Cosmic Ray Physics," Nucl. Phys. Proc. Suppl., vol. 212213, pp. 270-276, 2011. 
[315] F. O. Duraes, F. S. Navarra, and G. Wilk, "The Interacting gluon model: A Review," Braz. J. Phys., vol. 35, pp. 3-23, 2005.

[316] G. H. Arakelyan, C. Merino, C. Pajares, and Yu. M. Shabelski, "Feynman scaling violation on baryon spectra in pp collisions at LHC and cosmic ray energies," Phys. Atom. Nucl., vol. 76, pp. 316-325, 2013.

[317] A. Andronic et al., "Heavy-flavour and quarkonium production in the LHC era: from protonproton to heavy-ion collisions," Eur. Phys. J., vol. C76, no. 3, p. 107, 2016.

[318] D. Kharzeev and K. Tuchin, "Open charm production in heavy ion collisions and the color glass condensate," Nucl. Phys., vol. A735, pp. 248-266, 2004.

[319] K. Tuchin, "Heavy quark production by a quasiclassical color field in proton nucleus collisions," Phys. Lett., vol. B593, pp. 66-74, 2004.

[320] K. Tuchin, "Forward hadron production in high energy pA collisions: From RHIC to LHC," Nucl. Phys., vol. A798, pp. 61-73, 2008.

[321] Y. V. Kovchegov and K. Tuchin, "Production of q anti-q pairs in proton-nucleus collisions at high energies," Phys. Rev., vol. D74, p. 054014, 2006.

[322] H. Fujii, F. Gelis, and R. Venugopalan, "Heavy flavor production in pA collisions," J. Phys., vol. G34, pp. S937-942, 2007.

[323] E. R. Cazaroto, V. P. Goncalves, and F. S. Navarra, "Heavy quark production at LHC in the color dipole formalism," Nucl. Phys., vol. A872, pp. 196-209, 2011.

[324] H. Fujii and K. Watanabe, "Heavy quark pair production in high energy pA collisions: Open heavy flavors," Nucl. Phys., vol. A920, pp. 78-93, 2013.

[325] T. Altinoluk, N. Armesto, G. Beuf, A. Kovner, and M. Lublinsky, "Heavy quarks in proton-nucleus collisions - the hybrid formalism," Phys. Rev., vol. D93, no. 5, p. 054049, 2016. 
[326] V. P. Goncalves and M. V. T. Machado, "Saturation Physics in Ultra High Energy Cosmic Rays: Heavy Quark Production," JHEP, vol. 04, p. 028, 2007.

[327] R. Enberg, M. H. Reno, and I. Sarcevic, "Prompt neutrino fluxes from atmospheric charm," Phys. Rev., vol. D78, p. 043005, 2008.

[328] M. V. Garzelli, S. Moch, and G. Sigl, "Lepton fluxes from atmospheric charm revisited," JHEP, vol. 10, p. 115, 2015.

[329] A. Bhattacharya, R. Enberg, Y. S. Jeong, C. S. Kim, M. H. Reno, I. Sarcevic, and A. Stasto, "Prompt atmospheric neutrino fluxes: perturbative QCD models and nuclear effects," JHEP, vol. 11, p. 167, 2016.

[330] T. K. Gaisser and M. Honda, "Flux of atmospheric neutrinos," Ann. Rev. Nucl. Part. Sci., vol. 52, pp. 153-199, 2002.

[331] R. Laha and S. J. Brodsky, "IC at IC: IceCube can constrain the intrinsic charm of the proton," 2016.

[332] F. Halzen and L. Wille, "Charm contribution to the atmospheric neutrino flux," Phys. Rev., vol. D94, no. 1, p. 014014, 2016.

[333] F. Halzen and L. Wille, "Upper Limit on Forward Charm Contribution to Atmospheric Neutrino Flux," 2016.

[334] T. Gaisser and F. Halzen, "IceCube," Ann. Rev. Nucl. Part. Sci., vol. 64, pp. 101-123, 2014.

[335] M. G. Aartsen et al., "Measurement of the Atmospheric $\nu_{e}$ Spectrum with IceCube," Phys. Rev., vol. D91, p. 122004, 2015.

[336] S. J. Brodsky, A. Kusina, F. Lyonnet, I. Schienbein, H. Spiesberger, and R. Vogt, "A review of the intrinsic heavy quark content of the nucleon," Adv. High Energy Phys., vol. 2015, p. 231547, 2015. 
[337] S. J. Brodsky, V. A. Bednyakov, G. I. Lykasov, J. Smiesko, and S. Tokar, "The Physics of Heavy Quark Distributions in Hadrons: Collider Tests," 2016.

[338] S. J. Brodsky, P. Hoyer, C. Peterson, and N. Sakai, "The Intrinsic Charm of the Proton," Phys. Lett., vol. B93, pp. 451-455, 1980.

[339] R. Vogt and S. J. Brodsky, "QCD and intrinsic heavy quark predictions for leading charm and beauty hadroproduction," Nucl. Phys., vol. B438, pp. 261$277,1995$.

[340] S. Paiva, M. Nielsen, F. S. Navarra, F. O. Duraes, and L. L. Barz, "Virtual meson cloud of the nucleon and intrinsic strangeness and charm," Mod. Phys. Lett., vol. A13, pp. 2715-2724, 1998.

[341] F. S. Navarra, M. Nielsen, C. A. A. Nunes, and M. Teixeira, "On the intrinsic charm component of the nucleon," Phys. Rev., vol. D54, pp. 842-846, 1996.

[342] F. M. Steffens, W. Melnitchouk, and A. W. Thomas, "Charm in the nucleon," Eur. Phys. J., vol. C11, pp. 673-683, 1999.

[343] F. Carvalho, F. O. Duraes, F. S. Navarra, and M. Nielsen, "Does the D+ / D- production asymmetry decrease at large x(F)?," Phys. Rev. Lett., vol. 86, pp. 5434-5437, 2001.

[344] J. Pumplin, H. L. Lai, and W. K. Tung, "The Charm Parton Content of the Nucleon," Phys. Rev., vol. D75, p. 054029, 2007.

[345] A. Aleedaneshvar, M. Goharipour, and S. Rostami, "The impact of intrinsic charm on the parton distribution functions," Eur. Phys. J., vol. A52, no. 12, p. $352,2016$.

[346] G. Ingelman and M. Thunman, "Signals for intrinsic charm in high-energy interactions," Z. Phys., vol. C73, pp. 505-515, 1997. 
[347] B. A. Kniehl, G. Kramer, I. Schienbein, and H. Spiesberger, "Open charm hadroproduction and the charm content of the proton," Phys. Rev., vol. D79, p. 094009, 2009.

[348] V. P. Goncalves and F. S. Navarra, "Looking for intrinsic charm in the forward region at BNL RHIC and CERN LHC," Nucl. Phys., vol. A842, pp. 59-71, 2010.

[349] S. J. Brodsky, B. Kopeliovich, I. Schmidt, and J. Soffer, "Diffractive Higgs production from intrinsic heavy flavors in the proton," Phys. Rev., vol. D73, p. $113005,2006$.

[350] V. A. Bednyakov, M. A. Demichev, G. I. Lykasov, T. Stavreva, and M. Stockton, "Searching for intrinsic charm in the proton at the LHC," Phys. Lett., vol. B728, pp. 602-606, 2014.

[351] P.-H. Beauchemin, V. A. Bednyakov, G. I. Lykasov, and Yu. Yu. Stepanenko, "Search for intrinsic charm in vector boson production accompanied by heavy flavor jets," Phys. Rev., vol. D92, no. 3, p. 034014, 2015.

[352] S. Dulat, T.-J. Hou, J. Gao, J. Huston, J. Pumplin, C. Schmidt, D. Stump, and C. P. Yuan, "Intrinsic Charm Parton Distribution Functions from CTEQ-TEA Global Analysis," Phys. Rev., vol. D89, no. 7, p. 073004, 2014.

[353] P. Jimenez-Delgado, T. J. Hobbs, J. T. Londergan, and W. Melnitchouk, "Reply to Comment on "New limits on intrinsic charm in the nucleon from global analysis of parton distributions"," Phys. Rev. Lett., vol. 116, no. 1, p. 019102 , 2016.

[354] S. J. Brodsky and S. Gardner, "Comment on "New Limits on Intrinsic Charm in the Nucleon from Global Analysis of Parton Distributions"," Phys. Rev. Lett., vol. 116, no. 1, p. 019101, 2016. 
[355] G. Bailas and V. P. Goncalves, "Phenomenological implications of the intrinsic charm in the $Z$ boson production at the LHC," Eur. Phys. J., vol. C76, no. 3, p. 105, 2016.

[356] T. Boettcher, P. Ilten, and M. Williams, "Direct probe of the intrinsic charm content of the proton," Phys. Rev., vol. D93, no. 7, p. 074008, 2016.

[357] A. Rakotozafindrabe et al., "Ultra-relativistic heavy-ion physics with AFTER@LHC," Nucl. Phys., vol. A904-905, pp. 957c-960c, 2013.

[358] J. P. Lansberg et al., "Prospects for A Fixed-Target ExpeRiment at the LHC: AFTER@LHC," PoS, vol. ICHEP2012, p. 547, 2013.

[359] J. P. Lansberg et al., "AFTER@LHC: a precision machine to study the interface between particle and nuclear physics," EPJ Web Conf., vol. 66, p. 11023, 2014.

[360] J.-P. Lansberg et al., "Physics case for a polarised target for AFTER@LHC," PoS, vol. PSTP2015, p. 042, 2016.

[361] "Aims of the project." http://after.in2p3.fr/after/index.php/Aims_of_ the_project. Accessed: 2017-04-11.

[362] F. Halzen, Y. S. Jeong, and C. S. Kim, "Charge Asymmetry of Weak Boson Production at the LHC and the Charm Content of the Proton," Phys. Rev., vol. D88, p. 073013, 2013.

[363] N. N. Nikolaev, G. Piller, and B. G. Zakharov, "Quantum coherence in heavy flavor production on nuclei," J. Exp. Theor. Phys., vol. 81, pp. 851-859, 1995. [Zh. Eksp. Teor. Fiz.108,1554(1995)].

[364] N. N. Nikolaev, G. Piller, and B. G. Zakharov, "Inclusive heavy flavor production from nuclei," Z. Phys., vol. A354, pp. 99-105, 1996.

[365] B. Z. Kopeliovich and A. V. Tarasov, "Gluon shadowing and heavy flavor production off nuclei," Nucl. Phys., vol. A710, pp. 180-217, 2002. 
[366] J. Raufeisen and J.-C. Peng, "Relating parton model and color dipole formulation of heavy quark hadroproduction," Phys. Rev., vol. D67, p. 054008, 2003.

[367] I. Abt et al., "Measurement of D0, D+, D+(s) and D*+ Production in Fixed Target 920-GeV Proton-Nucleus Collisions," Eur. Phys. J., vol. C52, pp. 531$542,2007$.

[368] L. Adamczyk et al., "Measurements of $D^{0}$ and $D^{*}$ Production in $p+p$ Collisions at $\sqrt{s}=200 \mathrm{GeV}, "$ Phys. Rev., vol. D86, p. 072013, 2012.

[369] B. Abelev et al., "Measurement of prompt $J / \psi$ and beauty hadron production cross sections at mid-rapidity in $p p$ collisions at $\sqrt{s}=7 \mathrm{TeV}$," JHEP, vol. 11, p. 065, 2012.

[370] R. Aaij et al., "Measurement of $J / \psi$ production in $p p$ collisions at $\sqrt{s}=7 \mathrm{TeV}$," Eur. Phys. J., vol. C71, p. 1645, 2011.

[371] R. Aaij et al., "Prompt charm production in pp collisions at sqrt(s)=7 TeV," Nucl. Phys., vol. B871, pp. 1-20, 2013.

[372] R. Aaij et al., "Measurements of prompt charm production cross-sections in $p p$ collisions at $\sqrt{s}=13 \mathrm{TeV}, "$ JHEP, vol. 03, p. 159, 2016. [Erratum: JHEP09,013(2016)].

[373] D. d'Enterria, "Forward Physics at the LHC: within and beyond the Standard Model," AIP Conf. Proc., vol. 1038, pp. 95-109, 2008.

[374] K. Akiba et al., "LHC Forward Physics," J. Phys., vol. G43, p. 110201, 2016.

[375] P. Bozek, A. Bzdak, and V. Skokov, "The rapidity dependence of the average transverse momentum in $\mathrm{p}+\mathrm{Pb}$ collisions at the LHC: the Color Glass Condensate versus hydrodynamics," Phys. Lett., vol. B728, pp. 662-665, 2014.

[376] B. B. Abelev et al., "Long-range angular correlations of $\pi, \mathrm{K}$ and $\mathrm{p}$ in $\mathrm{p}-\mathrm{Pb}$ collisions at $\sqrt{s_{\mathrm{NN}}}=5.02 \mathrm{TeV}, "$ Phys. Lett., vol. B726, pp. 164-177, 2013. 
[377] L. Adamczyk et al., "Effect of event selection on jetlike correlation measurement in $d+\mathrm{Au}$ collisions at $\sqrt{s_{\mathrm{NN}}}=200 \mathrm{GeV}$," Phys. Lett., vol. B743, pp. 333-339, 2015.

[378] B. Alver and G. Roland, "Collision geometry fluctuations and triangular flow in heavy-ion collisions," Phys. Rev., vol. C81, p. 054905, 2010. [Erratum: Phys. Rev.C82,039903(2010)].

[379] L. Adamczyk et al., "Third Harmonic Flow of Charged Particles in Au+Au Collisions at sqrtsNN = $200 \mathrm{GeV}, "$ Phys. Rev., vol. C88, no. 1, p. 014904, 2013.

[380] P. Bozek, "Elliptic flow in proton-proton collisions at $\operatorname{sqrt}(S)=7 \mathrm{TeV}$," Eur. Phys. J., vol. C71, p. 1530, 2011.

[381] T. Lappi, "Azimuthal harmonics of color fields in a high energy nucleus," Phys. Lett., vol. B744, pp. 315-319, 2015.

[382] B. Schenke, S. Schlichting, and R. Venugopalan, "Azimuthal anisotropies in p $+\mathrm{Pb}$ collisions from classical YangMills dynamics," Phys. Lett., vol. B747, pp. 76-82, 2015.

[383] T. Lappi, B. Schenke, S. Schlichting, and R. Venugopalan, "Tracing the origin of azimuthal gluon correlations in the color glass condensate," JHEP, vol. 01, p. 061, 2016.

[384] K. Aamodt et al., "Transverse momentum spectra of charged particles in protonproton collisions at $\sqrt{s}=900 \mathrm{GeV}$ with ALICE at the LHC," Phys. Lett., vol. B693, pp. 53-68, 2010.

[385] B. B. Abelev et al., "Energy Dependence of the Transverse Momentum Distributions of Charged Particles in pp Collisions Measured by ALICE," Eur. Phys. J., vol. C73, no. 12, p. 2662, 2013. 
[386] G. Aad et al., "Charged-particle multiplicities in $p p$ interactions at $\sqrt{s}=900$ GeV measured with the ATLAS detector at the LHC," Phys. Lett., vol. B688, pp. 21-42, 2010.

[387] G. Aad et al., "Charged-particle multiplicities in pp interactions measured with the ATLAS detector at the LHC," New J. Phys., vol. 13, p. 053033, 2011.

[388] V. Khachatryan et al., "Transverse momentum and pseudorapidity distributions of charged hadrons in pp collisions at $\sqrt{s}=0.9$ and $2.36 \mathrm{TeV}, " J H E P$, vol. 02, p. 041, 2010.

[389] V. Khachatryan et al., "Transverse-momentum and pseudorapidity distributions of charged hadrons in $p p$ collisions at $\sqrt{s}=7 \mathrm{TeV}$," Phys. Rev. Lett., vol. 105, p. $022002,2010$.

[390] S. Chatrchyan et al., "Charged particle transverse momentum spectra in $p p$ collisions at $\sqrt{s}=0.9$ and $7 \mathrm{TeV}, " J H E P$, vol. 08, p. 086, 2011.

[391] J. Kuokkanen, K. Rummukainen, and H. Weigert, "HERA-Data in the Light of Small x Evolution with State of the Art NLO Input," Nucl. Phys., vol. A875, pp. 29-93, 2012.

[392] T. Lappi and H. Mntysaari, "Direct numerical solution of the coordinate space Balitsky-Kovchegov equation at next to leading order," Phys. Rev., vol. D91, no. 7, p. 074016, 2015.

[393] J. Jalilian-Marian and A. H. Rezaeian, "Hadron production in pA collisions at the LHC from the Color Glass Condensate," Phys. Rev., vol. D85, p. 014017, 2012.

[394] A. H. Rezaeian, "CGC predictions for $\mathrm{p}+\mathrm{A}$ collisions at the LHC and signature of QCD saturation," Phys. Lett., vol. B718, pp. 1058-1069, 2013. 
[395] P. Tribedy and R. Venugopalan, "QCD saturation at the LHC: Comparisons of models to $\mathrm{p}+\mathrm{p}$ and $\mathrm{A}+\mathrm{A}$ data and predictions for $\mathrm{p}+\mathrm{Pb}$ collisions," Phys. Lett., vol. B710, pp. 125-133, 2012. [Erratum: Phys. Lett.B718,1154(2013)].

[396] G. P. Salam, "A Resummation of large subleading corrections at small x," JHEP, vol. 07, p. 019, 1998.

[397] M. Ciafaloni, D. Colferai, and G. P. Salam, "Renormalization group improved small x equation," Phys. Rev., vol. D60, p. 114036, 1999.

[398] B. Abelev et al., "Neutral pion and $\eta$ meson production in proton-proton collisions at $\sqrt{s}=0.9 \mathrm{TeV}$ and $\sqrt{s}=7 \mathrm{TeV}$," Phys. Lett., vol. B717, pp. 162-172, 2012 .

[399] B. I. Abelev et al., "Longitudinal double-spin asymmetry and cross section for inclusive neutral pion production at midrapidity in polarized proton collisions at $\sqrt{s}=200 \mathrm{GeV}, "$ Phys. Rev., vol. D80, p. 111108, 2009.

[400] J. Adams et al., "Evidence from d + Au measurements for final state suppression of high $\mathrm{p}(\mathrm{T})$ hadrons in $\mathrm{Au}+\mathrm{Au}$ collisions at RHIC," Phys. Rev. Lett., vol. 91, p. $072304,2003$.

[401] I. Arsene et al., "On the evolution of the nuclear modification factors with rapidity and centrality in $\mathrm{d}+\mathrm{Au}$ collisions at $\mathrm{s}(\mathrm{NN}) * *(1 / 2)=200-\mathrm{GeV}, "$ Phys. Rev. Lett., vol. 93, p. 242303, 2004.

[402] J. Adams et al., "Forward neutral pion production in $\mathrm{p}+\mathrm{p}$ and $\mathrm{d}+\mathrm{Au}$ collisions at $\mathrm{s}(\mathrm{NN}) * *(1 / 2)=200-\mathrm{GeV}, "$ Phys. Rev. Lett., vol. 97, p. 152302, 2006.

[403] J. Breitweg et al., "Measurement of the proton structure function F2 and sigmatot (gamma* p) at low $\mathrm{q}^{* *} 2$ and very low x at HERA," Phys. Lett., vol. B407, pp. 432-448, 1997. 
[404] J. Breitweg et al., "Measurement of the proton structure function $\mathrm{F}(2)$ at very low Q**2 at HERA," Phys. Lett., vol. B487, pp. 53-73, 2000.

[405] V. P. Goncalves and M. L. L. da Silva, "Probing the Color Glass Condensate in $p p$ collisions at forward rapidities and very low transverse momenta," Nucl. Phys., vol. A906, pp. 28-36, 2013.

[406] P. Tribedy and R. Venugopalan, "Saturation models of HERA DIS data and inclusive hadron distributions in $\mathrm{p}+\mathrm{p}$ collisions at the LHC," Nucl. Phys., vol. A850, pp. 136-156, 2011. [Erratum: Nucl. Phys.A859,185(2011)].

[407] B. Schenke, P. Tribedy, and R. Venugopalan, "Multiplicity distributions in p+p, p+A and A+A collisions from Yang-Mills dynamics," Phys. Rev., vol. C89, no. 2, p. 024901, 2014.

[408] S. J. Brodsky, G. P. Lepage, and P. B. Mackenzie, "On the Elimination of Scale Ambiguities in Perturbative Quantum Chromodynamics," Phys. Rev., vol. D28, p. $228,1983$.

[409] E. Levin and A. H. Rezaeian, "Hadron multiplicity in pp and AA collisions at LHC from the Color Glass Condensate," Phys. Rev., vol. D82, p. 054003, 2010.

[410] A. Kormilitzin and E. Levin, "Non-linear equation: Energy conservation and impact parameter dependence," Nucl. Phys., vol. A849, pp. 98-119, 2011.

[411] C. Contreras, E. Levin, and I. Potashnikova, "CGC/saturation approach: a new impact-parameter dependent model," Nucl. Phys., vol. A948, pp. 1-18, 2016.

[412] J. Berger and A. Stasto, "Numerical solution of the nonlinear evolution equation at small $\mathrm{x}$ with impact parameter and beyond the LL approximation," Phys. Rev., vol. D83, p. 034015, 2011.

[413] J. Berger and A. M. Stasto, "Small x nonlinear evolution with impact parameter and the structure function data," Phys. Rev., vol. D84, p. 094022, 2011. 
[414] J. Berger and A. M. Stasto, "Exclusive vector meson production and small-x evolution," JHEP, vol. 01, p. 001, 2013.

[415] B. Andersson, P. Dahlqvist, and G. Gustafson, "ON LOCAL PARTON HADRON DUALITY. 1. MULTIPLICITY," Z. Phys., vol. C44, p. 455, 1989.

[416] B. Andersson, P. Dahlqvist, and G. Gustafson, "ON LOCAL PARTON - HADRON DUAlity. 2. MOMEnTUM DiSTRIBUtions," Z. Phys., vol. C44, p. $461,1989$.

[417] Y. L. Dokshitzer, V. A. Khoze, and S. I. Troian, "On the concept of local parton hadron duality," J. Phys., vol. G17, pp. 1585-1587, 1991.

[418] S. J. Brodsky and G. R. Farrar, "Scaling Laws at Large Transverse Momentum," Phys. Rev. Lett., vol. 31, pp. 1153-1156, 1973.

[419] V. A. Matveev, R. M. Muradian, and A. N. Tavkhelidze, "Automodellism in the large - angle elastic scattering and structure of hadrons," Lett. Nuovo Cim., vol. 7, pp. 719-723, 1973.

[420] K. Aamodt et al., "Charged-particle multiplicity measurement in proton-proton collisions at $\sqrt{s}=0.9$ and $2.36 \mathrm{TeV}$ with ALICE at LHC," Eur. Phys. J., vol. C68, pp. 89-108, 2010.

[421] I. Arsene et al., "Centrality dependence of charged particle pseudorapidity distributions from d+Au collisions at $\mathrm{s}(\mathrm{NN})^{* *}(1 / 2)=200-\mathrm{GeV}$," Phys. Rev. Lett., vol. 94, p. 032301, 2005.

[422] B. Alver et al., "Phobos results on charged particle multiplicity and pseudorapidity distributions in $\mathrm{Au}+\mathrm{Au}, \mathrm{Cu}+\mathrm{Cu}, \mathrm{d}+\mathrm{Au}$, and $\mathrm{p}+\mathrm{p}$ collisions at ultrarelativistic energies," Phys. Rev., vol. C83, p. 024913, 2011.

[423] B. Abelev et al., "Pseudorapidity density of charged particles in $p+\mathrm{Pb}$ collisions at $\sqrt{s_{N N}}=5.02 \mathrm{TeV}, "$ Phys. Rev. Lett., vol. 110, no. 3, p. 032301, 2013. 
[424] B. B. Back et al., "Charged particle pseudorapidity density distributions from $\mathrm{Au}+\mathrm{Au}$ collisions at $\sqrt{s_{N N}}=130-\mathrm{GeV}, "$ Phys. Rev. Lett., vol. 87, p. 102303, 2001.

[425] B. B. Back et al., "The Significance of the fragmentation region in ultrarelativistic heavy ion collisions," Phys. Rev. Lett., vol. 91, p. 052303, 2003.

[426] E. Abbas et al., "Centrality dependence of the pseudorapidity density distribution for charged particles in Pb-Pb collisions at $\sqrt{s_{\mathrm{NN}}}=2.76 \mathrm{TeV}$," Phys. Lett., vol. B726, pp. 610-622, 2013.

[427] S. Chatrchyan et al., "Dependence on pseudorapidity and centrality of charged hadron production in $\mathrm{PbPb}$ collisions at a nucleon-nucleon centre-of-mass energy of $2.76 \mathrm{TeV}, " J H E P$, vol. 08, p. 141, 2011.

[428] B. B. Back et al., "Centrality dependence of the charged particle multiplicity near mid-rapidity in $\mathrm{Au}+\mathrm{Au}$ collisions at $\sqrt{s}(\mathrm{NN})=130-\mathrm{GeV}$ and $200-\mathrm{GeV}$," Phys. Rev., vol. C65, p. 061901, 2002.

[429] J. Whitmore, "Experimental Results on Strong Interactions in the NAL Hydrogen Bubble Chamber," Phys. Rept., vol. 10, pp. 273-373, 1974.

[430] W. Thome et al., "Charged Particle Multiplicity Distributions in p p Collisions at ISR Energies," Nucl. Phys., vol. B129, p. 365, 1977.

[431] G. J. Alner et al., "Scaling of Pseudorapidity Distributions at c.m. Energies Up to 0.9-TeV," Z. Phys., vol. C33, pp. 1-6, 1986.

[432] R. Nouicer et al., "Pseudorapidity distributions of charged particles in $\mathrm{d}+\mathrm{Au}$ and $\mathrm{p}+\mathrm{p}$ collisions at $\mathrm{s}(\mathrm{NN})^{* *}(1 / 2)=200-\mathrm{GeV}, " J$. Phys., vol. G30, pp. S1133S1138, 2004. 
[433] K. Aamodt et al., "Charged-particle multiplicity measurement in proton-proton collisions at $\sqrt{s}=7 \mathrm{TeV}$ with ALICE at LHC," Eur. Phys. J., vol. C68, pp. 345$354,2010$.

[434] C. Albajar et al., "A Study of the General Characteristics of $p \bar{p}$ Collisions at $\sqrt{s}$ =0.2-TeV to 0.9-TeV," Nucl. Phys., vol. B335, pp. 261-287, 1990.

[435] B. I. Abelev et al., "Systematic Measurements of Identified Particle Spectra in $p p, d^{+} \mathrm{Au}$ and $\mathrm{Au}+\mathrm{Au}$ Collisions from STAR," Phys. Rev., vol. C79, p. 034909, 2009.

[436] F. Abe et al., "Pseudorapidity distributions of charged particles produced in $\bar{p} p$ interactions at $\sqrt{s}=630 \mathrm{GeV}$ and $1800 \mathrm{GeV}, "$ Phys. Rev., vol. D41, p. 2330, 1990. [,119(1989)].

[437] I. G. Bearden et al., "Charged particle densities from $\mathrm{Au}+\mathrm{Au}$ collisions at $\mathrm{s}(\mathrm{NN}) * *(1 / 2)=130-\mathrm{GeV}, "$ Phys. Lett., vol. B523, pp. 227-233, 2001.

[438] I. G. Bearden et al., "Pseudorapidity distributions of charged particles from $\mathrm{Au}+\mathrm{Au}$ collisions at the maximum RHIC energy," Phys. Rev. Lett., vol. 88, p. 202301, 2002.

[439] S. S. Adler et al., "Systematic studies of the centrality and $\mathrm{s}(\mathrm{NN})^{* *}(1 / 2)$ dependence of the $\mathrm{d} E(\mathrm{~T}) / \mathrm{d}$ eta and $\mathrm{d}(\mathrm{N}(\mathrm{ch}) / \mathrm{d}$ eta in heavy ion collisions at mid-rapidity," Phys. Rev., vol. C71, p. 034908, 2005. [Erratum: Phys. Rev.C71,049901(2005)].

[440] G. Aad et al., "Measurement of the centrality dependence of the charged particle pseudorapidity distribution in lead-lead collisions at $\sqrt{s_{N N}}=2.76 \mathrm{TeV}$ with the ATLAS detector," Phys. Lett., vol. B710, pp. 363-382, 2012.

[441] S. Voloshin and Y. Zhang, "Flow study in relativistic nuclear collisions by Fourier expansion of Azimuthal particle distributions," Z. Phys., vol. C70, pp. 665-672, 1996. 
[442] A. M. Poskanzer and S. A. Voloshin, "Methods for analyzing anisotropic flow in relativistic nuclear collisions," Phys. Rev., vol. C58, pp. 1671-1678, 1998.

[443] N. Borghini, P. M. Dinh, and J.-Y. Ollitrault, "Flow analysis from multiparticle azimuthal correlations," Phys. Rev., vol. C64, p. 054901, 2001.

[444] Y. V. Kovchegov and K. L. Tuchin, "Elliptic flow from minijet production in heavy ion collisions," Nucl. Phys., vol. A708, pp. 413-434, 2002.

[445] J. Adams et al., "Azimuthal anisotropy in Au+Au collisions at $\mathrm{s}(\mathrm{NN}) * *(1 / 2)=$ 200-GeV," Phys. Rev., vol. C72, p. 014904, 2005.

[446] A. Bzdak, P. Bozek, and L. McLerran, "Fluctuation induced equality of multiparticle eccentricities for four or more particles," Nucl. Phys., vol. A927, pp. 15$23,2014$.

[447] L. Yan and J.-Y. Ollitrault, "Universal fluctuation-driven eccentricities in proton-proton, proton-nucleus and nucleus-nucleus collisions," Phys. Rev. Lett., vol. 112, p. 082301, 2014.

[448] A. Bzdak and V. Skokov, "Multi-particle eccentricities in collisions dominated by fluctuations," Nucl. Phys., vol. A943, pp. 1-8, 2015.

[449] J. Noronha and A. Dumitru, "Azimuthal asymmetries in high-energy collisions of protons with holographic shockwaves," Phys. Rev., vol. D89, no. 9, p. 094008, 2014 .

[450] Y. V. Kovchegov and M. D. Sievert, "A New Mechanism for Generating a Single Transverse Spin Asymmetry," Phys. Rev., vol. D86, p. 034028, 2012. [Erratum: Phys. Rev.D86,079906(2012)].

[451] T. Altinoluk, N. Armesto, G. Beuf, M. Martínez, and C. A. Salgado, "Next-toeikonal corrections in the CGC: gluon production and spin asymmetries in $\mathrm{pA}$ collisions," JHEP, vol. 07, p. 068, 2014. 
[452] T. Altinoluk and A. Kovner, "Particle Production at High Energy and Large Transverse Momentum - 'The Hybrid Formalism' Revisited," Phys. Rev., vol. D83, p. 105004, 2011.

[453] G. A. Chirilli, B.-W. Xiao, and F. Yuan, "One-loop Factorization for Inclusive Hadron Production in $p A$ Collisions in the Saturation Formalism," Phys. Rev. Lett., vol. 108, p. 122301, 2012.

[454] F. Gelis and A. Peshier, "Probing colored glass via q anti-q photoproduction," Nucl. Phys., vol. A697, pp. 879-901, 2002.

[455] A. Dumitru, T. Lappi, and L. McLerran, "Are the angular correlations in $p A$ collisions due to a Glasmion or Bose condensation?," Nucl. Phys., vol. A922, pp. 140-149, 2014. 